\title{
QUALITATIVE ASSESSMENT OF COMPOSITION AND RESERVOIR QUALITY OF ALBIAN-CENOMANIAN NANUSHUK FORMATION SANDSTONES, MEASURED OUTCROP SECTIONS, CENTRAL NORTH SLOPE, ALASKA
}

Kenneth P. Helmold, David L. LePain, and Nina T. Harun

Raw Data File 2021-13

This report has not been reviewed for technical content or for conformity to the editorial standards of DGGS.

2021

STATE OF ALASKA

DEPARTMENT OF NATURAL RESOURCES

DIVISION OF GEOLOGICAL \& GEOPHYSICAL SURVEYS
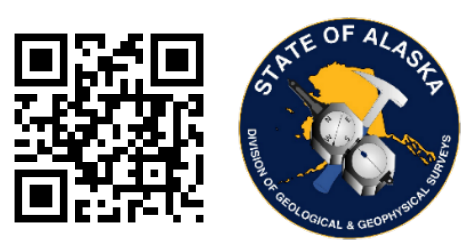


\section{STATE OF ALASKA}

Mike Dunleavy, Governor

\section{DEPARTMENT OF NATURAL RESOURCES}

Corri A. Feige, Commissioner

\section{DIVISION OF GEOLOGICAL \& GEOPHYSICAL SURVEYS}

Steve Masterman, State Geologist \& Director

Publications produced by the Division of Geological \& Geophysical Surveys are available to download from the DGGS website (dggs.alaska.gov). Publications on hard-copy or digital media can be examined or purchased in the Fairbanks office:

\section{Alaska Division of Geological \& Geophysical Surveys (DGGS)}

3354 College Road | Fairbanks, Alaska 99709-3707

Phone: 907.451.5010 | Fax 907.451.5050

dggspubs@alaska.gov $\mid$ dggs.alaska.gov

\section{DGGS publications are also available at:}

Alaska State Library, Historical

Collections \& Talking Book Center

395 Whittier Street

Juneau, Alaska 99801

Alaska Resource Library and

Information Services (ARLIS)

3150 C Street, Suite 100

Anchorage, Alaska 99503

\section{Suggested citation:}

Helmold, K.P., LePain, D.L., and Harun, N.T., 2021, Qualitative assessment of composition and reservoir quality of Albian-Cenomanian Nanushuk Formation sandstones, measured outcrop sections, central North Slope, Alaska: Alaska Division of Geological \& Geophysical Surveys Raw Data File 2021-13, 7 p. https://doi.org/10.14509/30746

This report was updated January 2022 to append the photomicrographs (figs. 2-101) to this booklet. These files are no longer available to download separately.
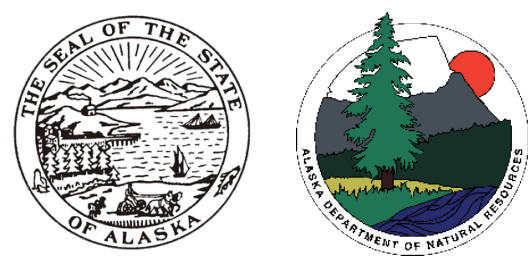


\section{QUALITATIVE ASSESSMENT OF COMPOSITION AND RESERVOIR QUALITY OF ALBIAN-CENOMANIAN NANUSHUK FORMATION SANDSTONES, MEASURED OUTCROP SECTIONS, CENTRAL NORTH SLOPE, ALASKA}

Kenneth P. Helmold ${ }^{1}$, David L. LePain², and Nina T. Harun²

\section{INTRODUCTION}

For the past 22 years, the Alaska Division of Geological \& Geophysical Surveys (DGGS) has studied outcrop exposures of the Albian-Cenomanian Nanushuk Formation on the North Slope of Alaska. This work involved detailed measurement of numerous stratigraphic sections (fig. 1A) with the goal of increasing our knowledge of the depositional environments, sedimentary facies, and stratal geometry of the Nanushuk. As part of this effort, samples from the measured sections were collected for subsequent laboratory study including the analysis of geochemistry, palynology, geochronology, petrology, and reservoir potential. Thin sections were routinely made from these samples with the goal of documenting their mineralogy, texture, and reservoir quality. When funds were available, samples were submitted for routine core analyses (determination of basic petrophysical properties including porosity, permeability, grain density —and in the case of subsurface samples-fluid saturation) and modal (point-count) analyses were performed on thin sections cut from the core plugs. Unfortunately, many of these thin sections have not undergone rigorous analysis. The primary goal of this study is to conduct a qualitative appraisal of thin sections collected from Nanushuk outcrops-particularly those that have not been previously studied-to ascertain their reservoir potential. Accompanying digital data available at: https://doi.org/10.14509/30746.

This work has been spurred by the hydrocarbon discoveries announced between 2015 and 2018 that are associated with stratigraphic traps in the Nanushuk Formation. These discoveries form two roughly parallel fairways; a western trend that includes the Willow and West Willow oil accumulations, located in the northeastern National Petroleum Reserve in Alaska, and an eastern trend comprised of the Pikka, Narwhal, and Horseshoe oil accumulations, located on state land in the Colville Delta area (fig. 1A). Recoverable resources are estimated to be more than 300 million barrels at Willow and West Willow, and 500 to 1200 million barrels at Pikka, Narwhal, and Horseshoe (Houseknecht, 2019).

\section{DATASET}

The DGGS sample collection was searched for petrographic thin sections collected from stratigraphic sections of the Nanushuk Formation measured by David LePain working with various colleagues, including Russ Kirkham, Paul Decker, Dave Houseknecht, and Nina Harun over the past 22 years. Simplified versions of some sections are included in LePain and others (2009; their app. A). The search yielded 264 thin sections of Nanushuk siltstone and sandstone-two of the thin sections are from grab samples collected by Paul Decker

\footnotetext{
${ }^{1}$ Alaska Division of Oil \& Gas, 550 W. $7^{\text {th }}$ Ave., Suite 800, Anchorage, AK 99501-3560; helmold@alaskan.com

${ }^{2}$ Alaska Division of Geological \& Geophysical Surveys, 3354 College Rd., Fairbanks, AK 99709-3707; david.lepain@alaska.gov
} 
and Bob Gillis that are not tied to the measured sections-and three additional thin sections of mudstone, siltstone, and sandstone of the Seabee Formation were collected from the upper portion of a measured section that straddles the Nanushuk - Seabee contact (section 00DL016 at Ninuluk Bluff). These 267 samples were collected from 32 stations located in eight geographic areas on the North Slope including Slope Mountain, May Creek, Arc Mountain, Rooftop Ridge, Big Bend of the Chandler River, Ninuluk Bluff on the Colville River, Killik Bend of the Colville River, and Horseshoe Bend of the Colville River (fig. 1B). The thin sections were examined qualitatively to ascertain several petrographic properties including grain size, detrital composition, cement and matrix content, abundance of burrows and laminations, and distribution of porosity. Sedimentary structures observed in the field were noted as was the interpreted sedimentary facies association. The results are compiled in a spreadsheet along with information on geographic location, latitude, longitude, and measured petrophysical properties (porosity, permeability, and grain density) of the few samples where available. Photomicrographs document key petrographic features of select samples.

Users may notice sample numbering and small location discrepancies throughout various North Slope publications. DGGS has completed significant recent work to assimilate sample metadata from various sources into comprehensive division-wide databases. Staff identified and, when possible, corrected sample labeling and location inconsistencies. The data table in this publication lists both the sample numbers written on each thin section and the standardized numbers used by the division databases. Thin sections and related sample material are archived by the Geological Materials Center in Anchorage, Alaska, and are available for public viewing on request.

Because sample collection spans several decades, the originating GPS coordinate system varies by year. This publication provides locations converted to the WGS84 coordinate system. Locations have been plotted using ESRI software and visually verified for accuracy. Location coordinates for samples collected along a measured stratigraphic section reflect the GPS reading at the base of the measured section.

\section{QUALITATIVE OBSERVATIONS}

\section{Grain size (grain_size)}

The mean grain size of each sample was estimated by using an ocular reticle to measure the long-axis length (in $\mathrm{mm}$ ) of several grains with dimensions in the mid-range of observed values. These measurements were then converted to the equivalent Wentworth size class. A single value is reported for well-sorted samples (e.g., vfl-lower very-fine sand), while for more poorly sorted samples a range of values (e.g., vfU-fL_upper very-fine to lower fine sand) is typically recorded. The majority of sandstone samples are very fine- to finegrained; several are medium-grained and a few are coarse-grained.

\section{Porosity (porosity)}

Porosity was determined by estimating the amount of blue-dyed epoxy visible in each thin section, with broad descriptive terms used to express its quantity and distribution. The terms used are: nil indicates porosity of $<5$ percent, moderate equates to 5-15 percent porosity, good designates $15-25$ percent porosity, and abundant indicates porosity $>25$ percent (table 1). Spotty is used to describe an uneven distribution of porosity that is qualitatively less than 10 percent thereby resulting in the sample having minimal reservoir potential. 
These estimates are intended to convey a general sense of the reservoir potential of a sample and are not meant to replace detailed laboratory measurements.

It should be kept in mind that visual porosity estimates are sometimes at odds with petrophysical properties measured in the laboratory, particularly for argillaceous samples. Sandstone with significant clay matrix or argillaceous lithic grains commonly has measured porosities as high as 8-10 percent while showing little to no traces of visible porosity in thin section. Clay minerals, particularly authigenic clays, contain significant micropores with pore-throat radii of $0.5 \mu \mathrm{m}$ or less (Wilson and Pittman, 1977; Nadeau and Hurst, 1991; Hurst and Nadeau, 1995) — dimensions considerably less than the standard thickness of a thin section $(30 \mu \mathrm{m})$. This porosity is detected in laboratory measurements but is not typically visible in thin section due to the dense packing of overlapping clay platelets.

\section{Mineralogy (mineralogy)}

Samples consist primarily very fine- to fine-grained sandstone with a fairly consistent set of framework components that include monocrystalline and polycrystalline quartz, chert, phyllite/schist, detrital carbonate, shale/mudstone, quartzite, plagioclase, and K-feldspar with minor quantities of organic matter and mica. Despite this homogenous composition, prior work (Helmold, 2016; Helmold and LePain, 2021) has shown that the composition of Nanushuk sandstone can vary as a function of grain size, with a greater chert content evident in medium-grained and coarser samples. The observations in column $U$ of appendix 1 highlight samples with very abundant, significant, or atypical grains. These samples contain significant quantities of chert, detrital carbonate, or argillaceous metamorphic rock fragments (MRFs), particularly those that are considerably deformed. The term "green mica" denotes a diverse group of phyllosilicates grains that includes biotite, chloritized biotite, degraded biotite, chlorite, and glauconite. They appear to be restricted largely to the shoreface facies association-and less commonly in the offshore transition facies-exposed at the Arc Mountain and Rooftop Ridge measured sections. It is unclear whether their distribution is controlled by depositional parameters (facies) or by provenance as suggested by their aerial distribution.

A few medium- to coarse-grained quartzose sandstones that consist largely of monocrystalline quartz appear at odds with the generally observed positive correlation between grain size and chert content. These samples consist of a greater proportion of monocrystalline quartz than chert and contain various amounts of silica cement in the form of quartz overgrowths, with some having good reservoir quality despite the presence of overgrowths. These quartzose sandstone samples lack significant quantities of argillaceous rock fragments, are at least partially cemented by quartz overgrowths, and are clearly on a different diagenetic pathway than the more argillaceous lithic- and chert- rich Nanushuk sandstone.

A cursory analysis of the data reveals that medium- to coarse-grained sandstone of the fluvial channel (FC) facies exposed at Arc Mountain is largely chert-rich. In contrast, similar sized sandstone of the distributary channel (DC) facies exposed at Arc and Slope Mountains consists of both chert- and monocrystalline quartz-rich sandstone. The latter variant may result from greater abrasion and selective removal of chert in the farther-transported marine sandstone. 

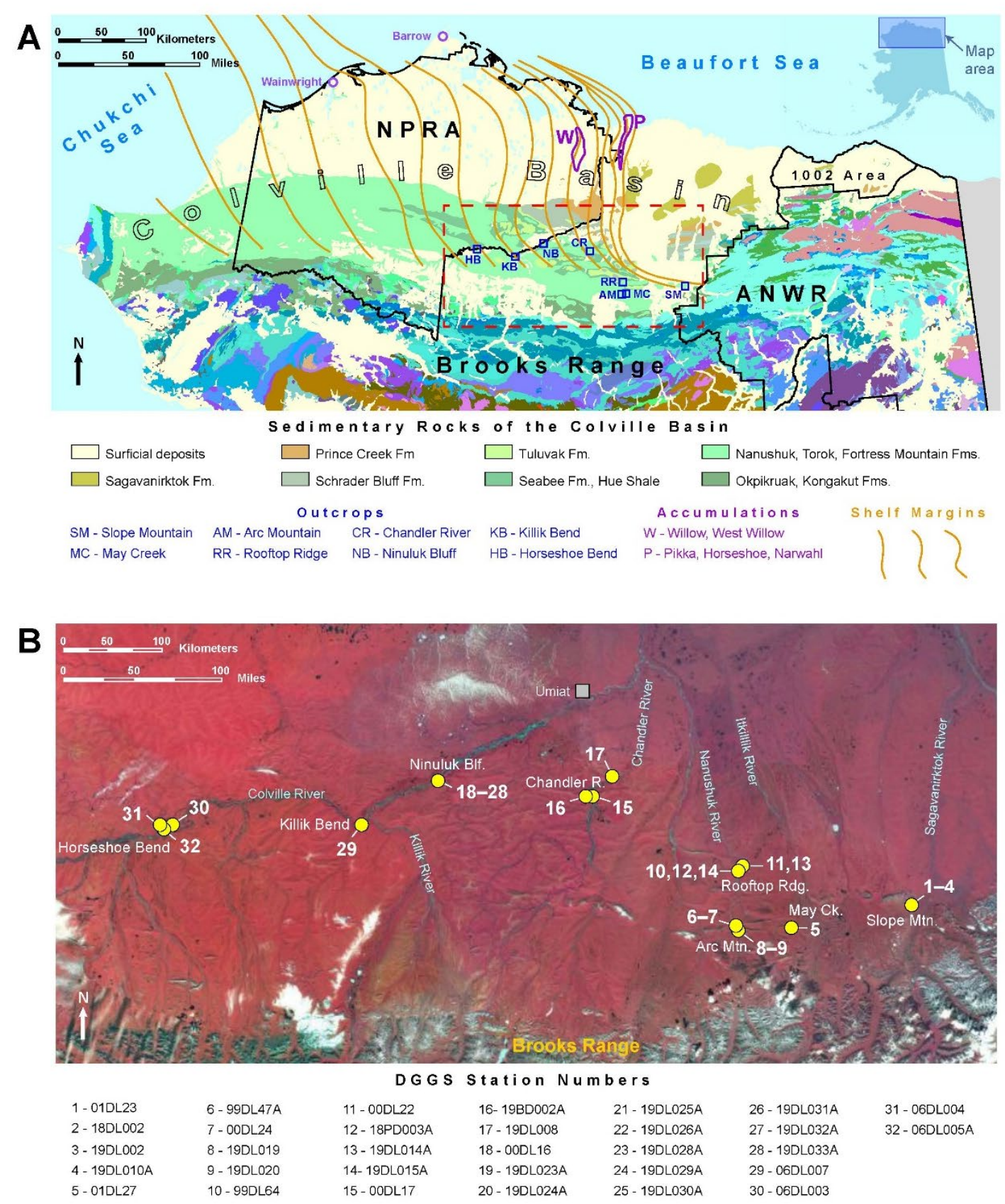

Figure 1. A. Geologic map of northern Alaska (from Wilson and others, 2015) showing the location of measured outcrop sections addressed in this report. Blue squares indicate outcrop sections: SM-Slope Mountain, MC-May Creek, AM-Arc Mountain, RR-Rooftop Ridge, CR-Chandler River, NB-Ninuluk Bluff, KB-Killik Bend, HB-Horseshoe Bend. Purple ovals show the locations of the Willow and West Willow (W) and Pikka, Horseshoe, and Narwahl (P) accumulations. Dashed red box outlines area displayed in B. Orange lines delineate the approximate locations of Nanushuk lowstand shelf margins (from Houseknecht, 2019). B. Color infrared aerial photographic image showing the location of the 32 DGGS stations from eight geographic areas discussed in this report. Most stations represent measured outcrop sections from which several samples have been collected. A few represent isolated grab samples. 
Table 1. Terms used to describe ranges in porosity observed in thin section.

\begin{tabular}{cc} 
Descriptive Term & Porosity Range (percent) \\
\hline nil & $<5$ \\
\hline moderate & $5-15$ \\
\hline good & $15-25$ \\
\hline abundant & $>25$
\end{tabular}

\section{Cement/Matrix (cement_matrix)}

The reduction of porosity in Nanushuk sandstone is largely accomplished by the mechanical deformation of ductile lithic grains resulting in minimal quantities of authigenic cement in most samples. However, some samples do contain significant quantities of carbonate cement, typically calcite, that largely destroys their reservoir potential. The distribution and texture of this cement suggest that it has a late-stage, replacement origin. Other minor cements include kaolinite, siderite, and Fe-oxide, the latter two commonly present as rims on detrital grains. A few samples contain silica cement in the form of quartz overgrowths, as noted above this cement appears largely restricted to sandstone with a higher detrital quartz content.

\section{Petrographic Features (petrographic_features)}

Miscellaneous petrographic features and/or structures that are noted include burrows, laminations, mineral veins, and fractures.

\section{Facies Association (facies_association)}

Fifteen lithofacies associations were recognized in outcrop that record a spectrum from fully marine (e.g., slope, offshore shelf) to marginal marine (e.g., prodelta, shoreface, delta-front) and nonmarine (e.g., crevasse channel, fluvial channel) depositional environments (table 2). Classifying crevasse channel, crevasse delta and splay deposits as nonmarine oversimplifies their depositional setting - these associations occur interbedded in both alluvial floodbasin (nonmarine) and interdistributary bayfill (marginal-marine) mudstone. Lithofacies associations were identified based on vertical stacking patterns of component facies, bed thickness, sedimentary structures, bioturbation, grain size, and sorting.

Table 2. Recognized facies associations and their codes.

\begin{tabular}{cc} 
Facies Association Code & Facies Association \\
\hline SL & Slope \\
\hline OFSH & Offshore shelf \\
\hline OFT & Offshore transition \\
\hline PD & Prodelta \\
\hline
\end{tabular}




\begin{tabular}{cc} 
Facies Association Code & Facies Association \\
\hline SF & Shoreface \\
\hline DF & Delta-front \\
\hline MB & Distributary mouth bar \\
\hline DC & Distributary channel \\
\hline TI & Tidal inlet \\
\hline IB & Interdistributary bay \\
\hline WO & Washoverfan \\
\hline CC & Crevasse channel \\
\hline CD & Crevasse delta \\
\hline FC & Fluvial channel \\
\hline S & Splay
\end{tabular}

\section{Sedimentary Structures (sedimentary_structures)}

Primary sedimentary structures include a myriad of features that were formed at the time of sediment deposition or shortly afterward while the detritus was still unlithified. Many of these structures originate by physical processes that involve a moving medium (water, wind) at the time of deposition. They are useful in interpreting the relative energy and flow direction of the depositing currents. Features formed after deposition include loading and slumping of unconsolidated sediment and biogenic modifications that consist of burrowing, boring, or grazing/feeding activities of organism. Classification of sedimentary structures can be based on whether the features are of depositional, erosional, deformational, or biogenic origin (Boggs, 2009). Some of the structures observed include horizontal laminations, cross bedding, hummocky cross stratification, swaley cross stratification, ripple cross laminations, root structures, and bioturbated sand.

\section{PHOTOMICROGRAPHS}

Photomicrographs acquired from 100 samples of Nanushuk siltstone and sandstone that were deemed representative of the larger collection of samples and one Seabee sandstone are shown in figures 2-102. They are presented in a consistent format to facilitate comparison. Each figure consists of four images taken at two different magnifications (50x and 100x total magnification) and with two different lighting conditions (planepolarized light $[\mathrm{PPL}]$ and polarized light with crossed polars $[\mathrm{XN}]$ ). The term crossed polars is synonymous with the outdated phrase crossed nicols which refers to the first polarizing prism originally designed by William Nicol in 1828 and used extensively in older petrographic microscopes-hence the abbreviation XN. Details of the four images are: A) = 50x magnification under PPL, 500 $\mu \mathrm{m}$ scale bar; B) $=50 \mathrm{x}$ magnification under $\mathrm{XN}, 500 \mu \mathrm{m}$ scale bar; C) $=100 \mathrm{x}$ magnification under PPL, $200 \mu \mathrm{m}$ scale bar; and D) $=100 \mathrm{x}$ magnification under $\mathrm{XN}, 200 \mu \mathrm{m}$ scale bar. The images taken with plane-polarized light (A and C) are most useful for observing overall rock texture (grain size and sorting), general grain composition, quantity and type 
(primary versus secondary) of porosity, and extent of bioturbation and laminations. The images taken with crossed polars are indispensable for recognizing chert which occurs in significant quantities in medium- to coarse-grained Nanushuk sandstone because it is difficult to distinguish from quartz with plane-polarized light. They are also useful for recognizing detrital carbonate grains (mostly dolomite) because their appearance in PPL and XN are similar due to extreme birefringence resulting in high-order white interference colors. The crossed polar images were taken using the High Dynamic Range (HDR) module of AxioVision, the Carl Zeiss software for acquiring digital images. They were subsequently processed with the HDR Toning algorithm in Adobe Photoshop to ensure grains with all levels of extinction have the appropriate exposure. 
Fig 002 - 01DL023-027.0

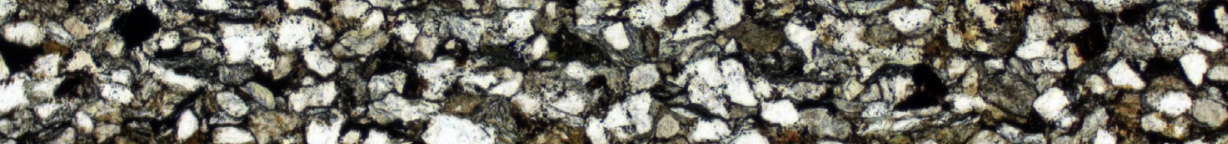

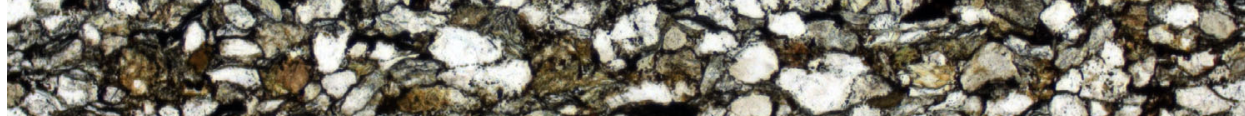

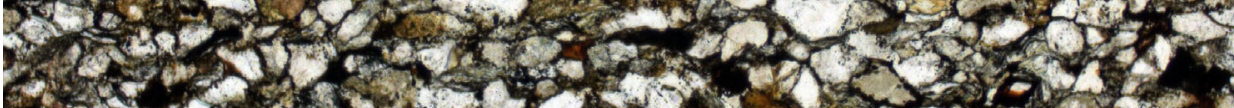

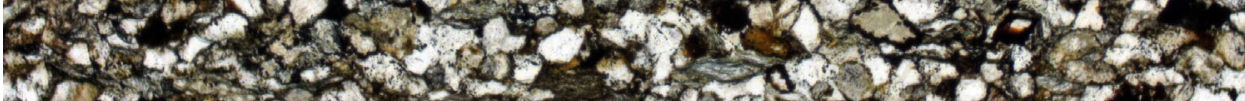

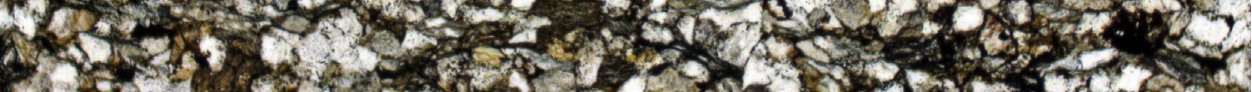

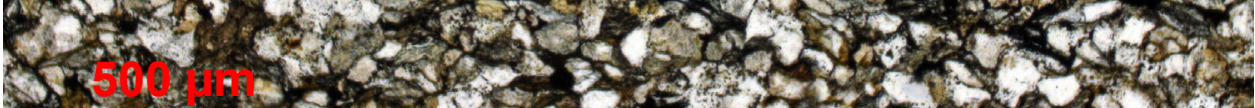

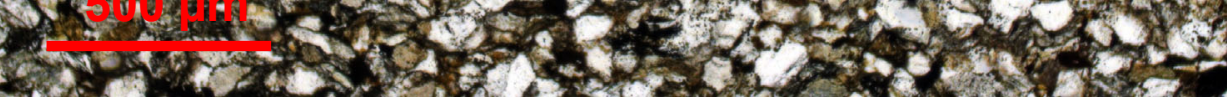

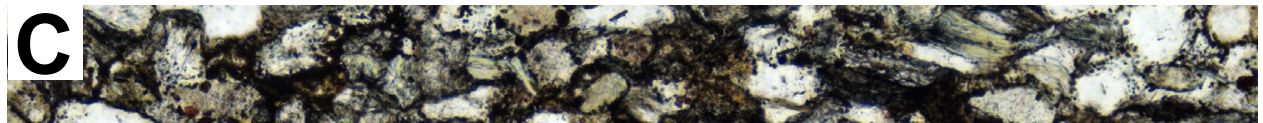

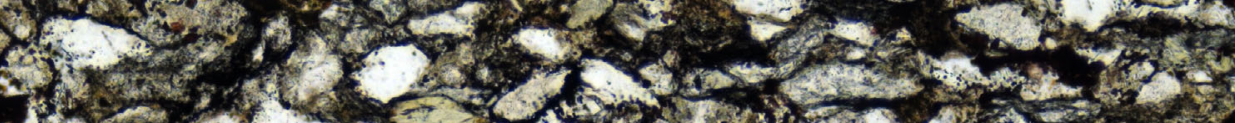

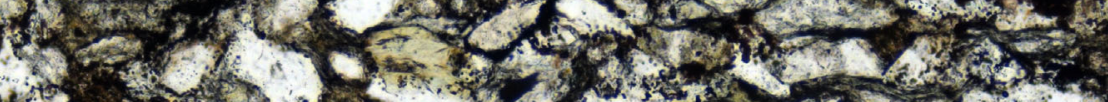

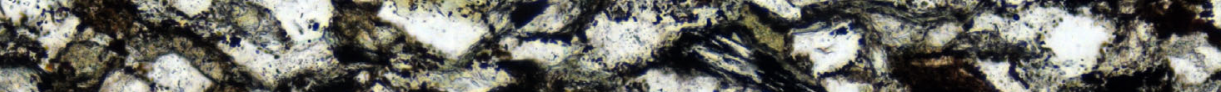

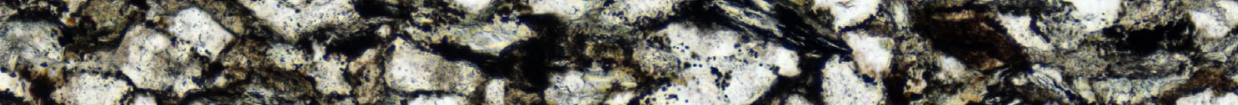
5270 , 3 . 3 ,

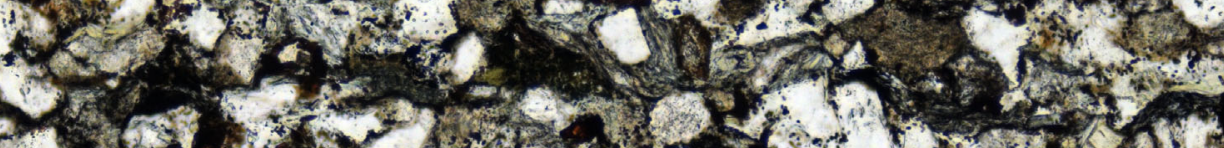

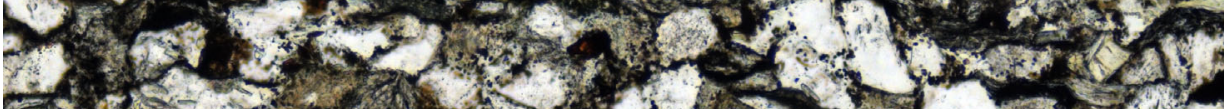

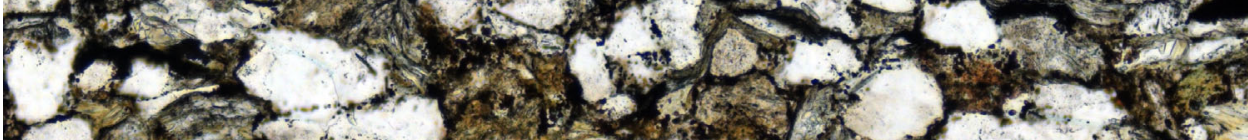

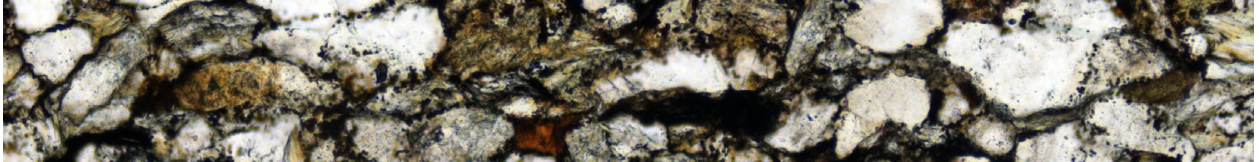

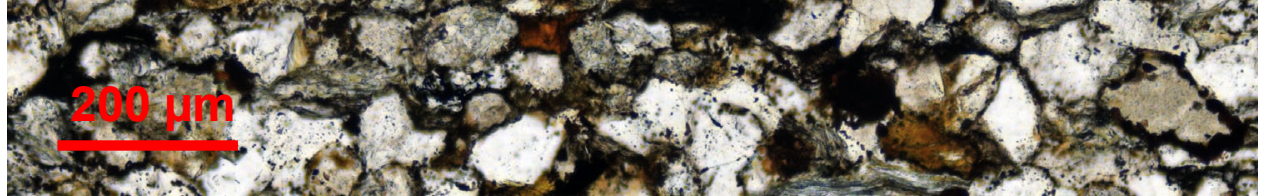
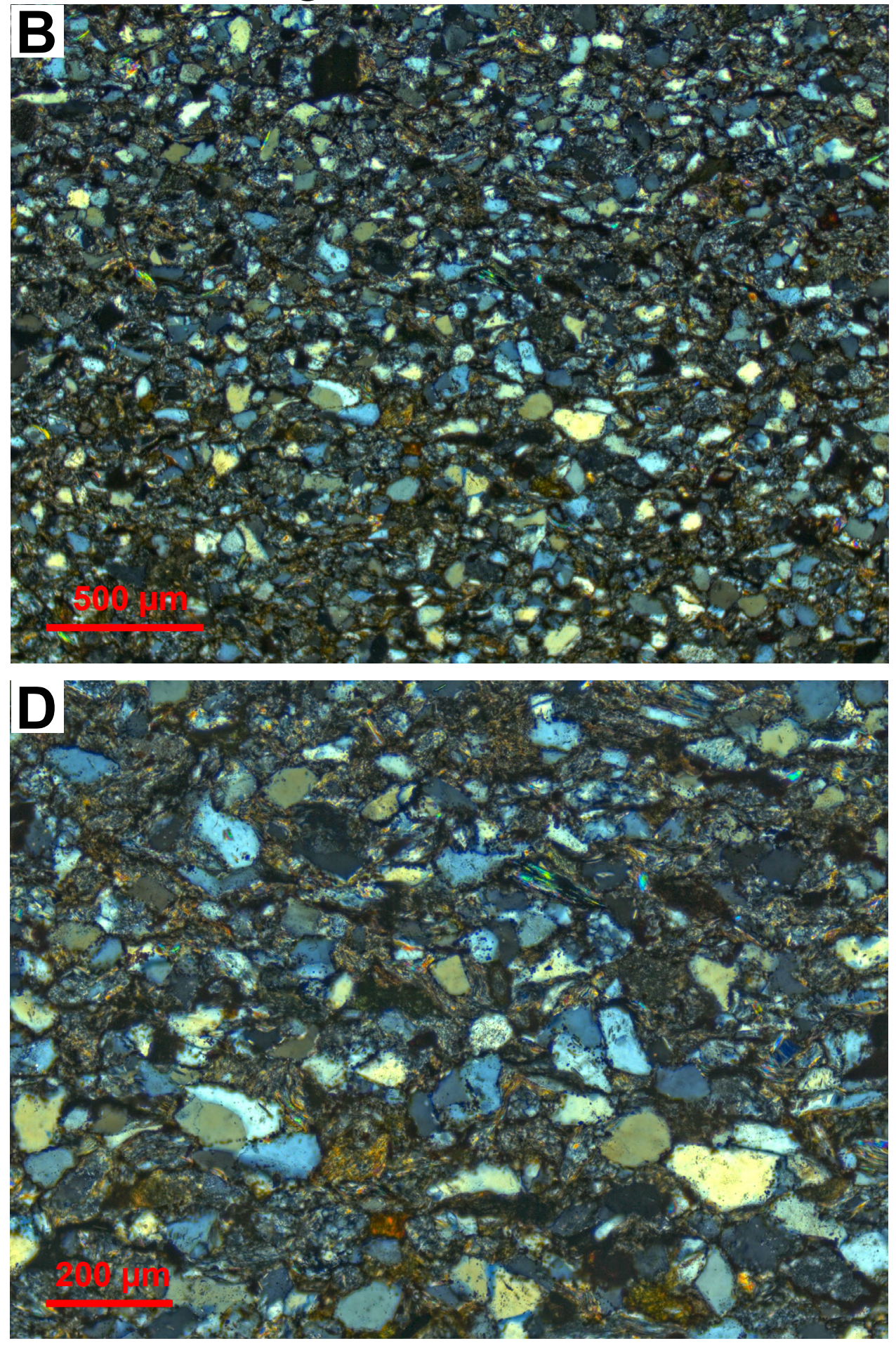
Fig 003 - 01DL023-037.1

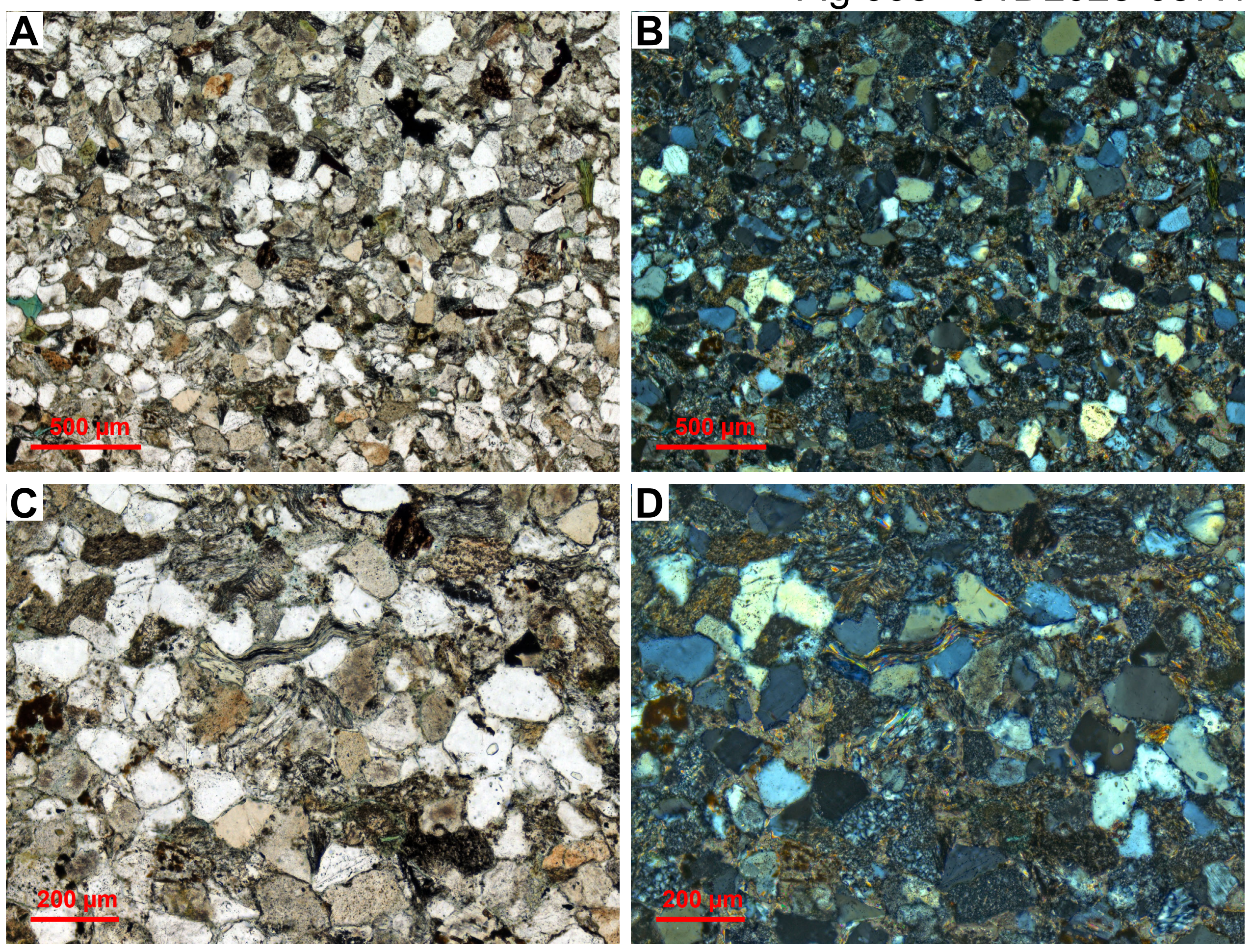


Fig 004 - 01DL023-148.0

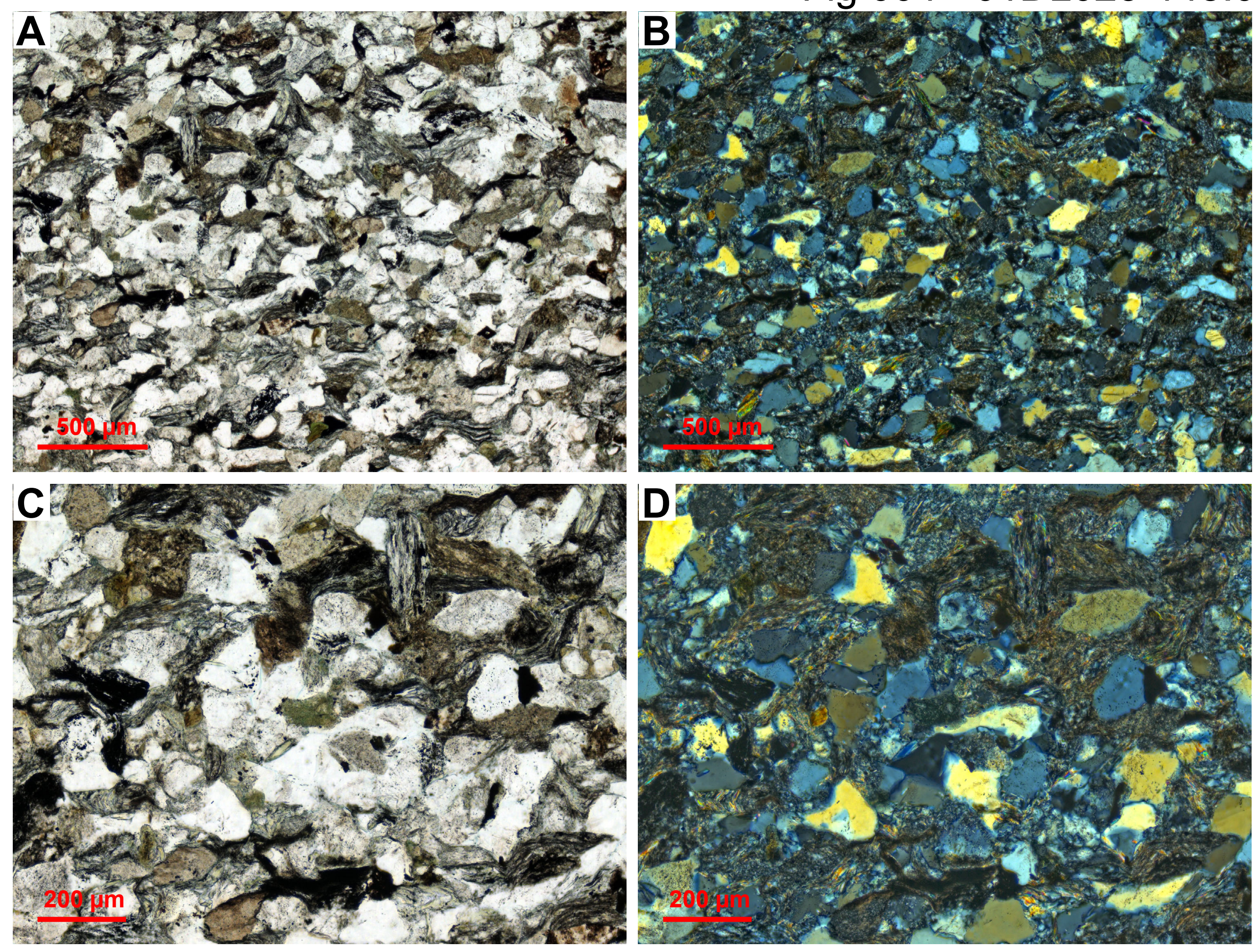


Fig 005 - 01DL023-240.6

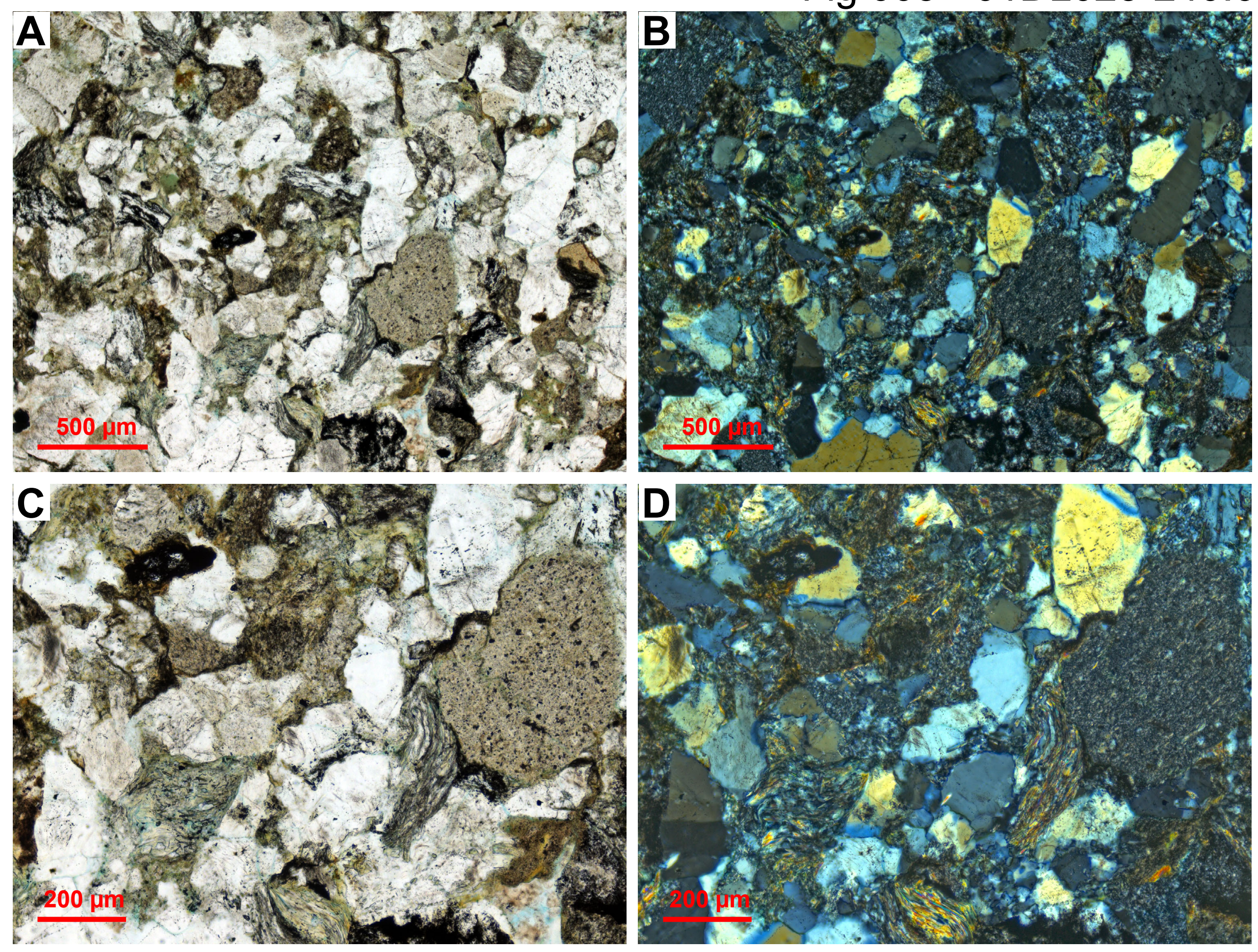


Fig 006 - 01DL023-249.8
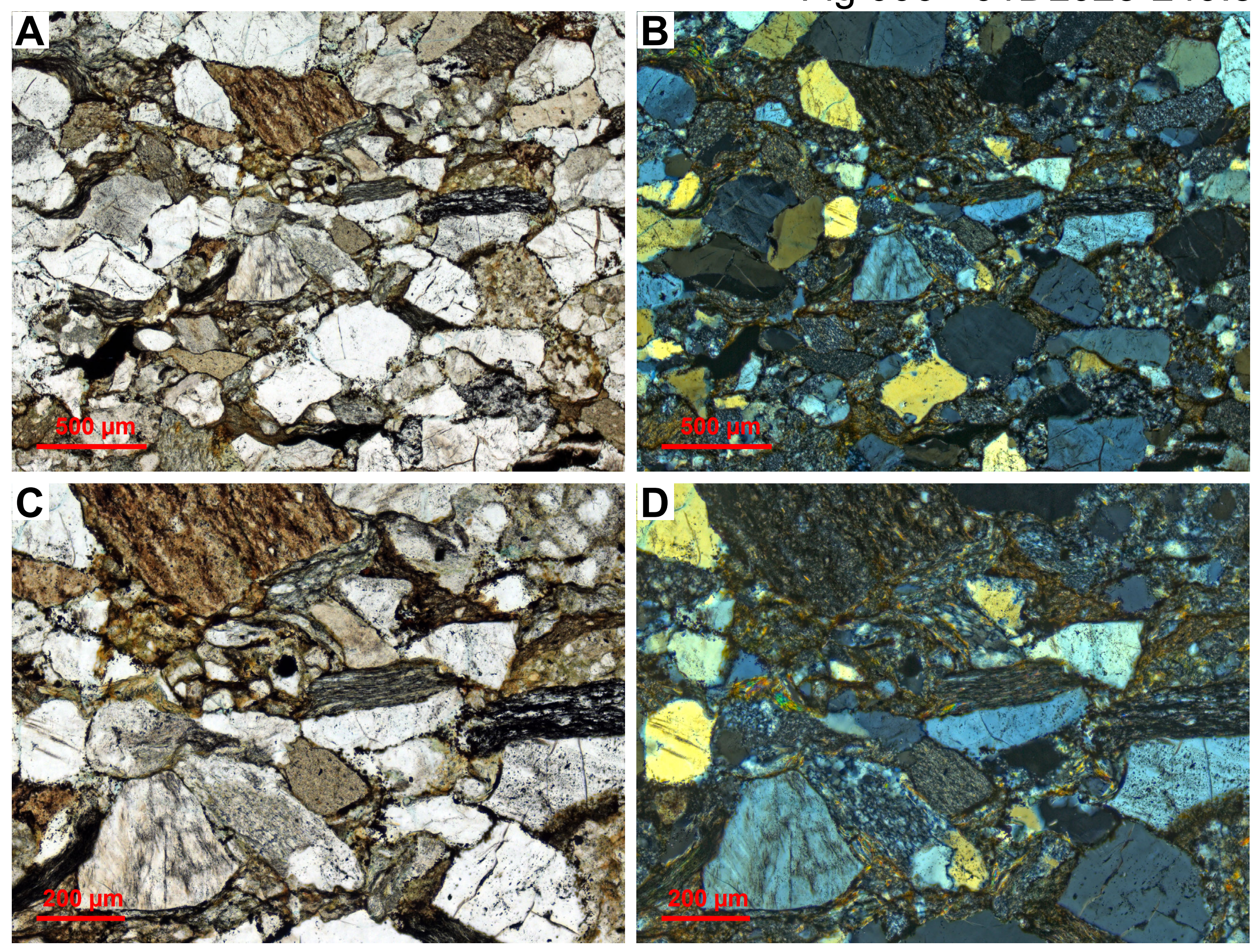
Fig 007 - 01DL023-305.0
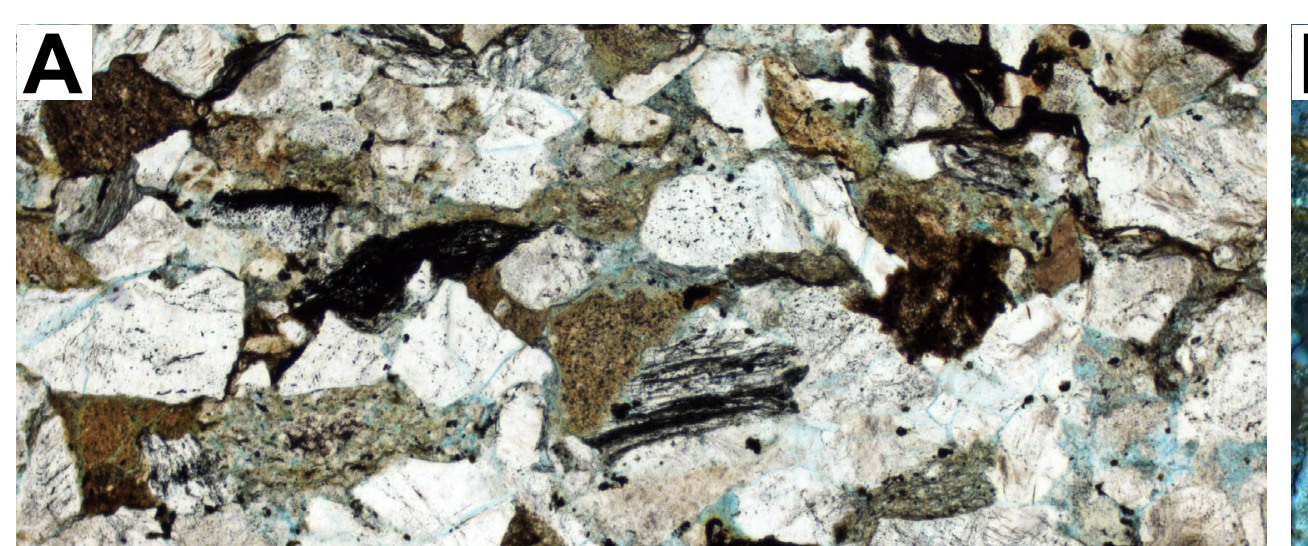

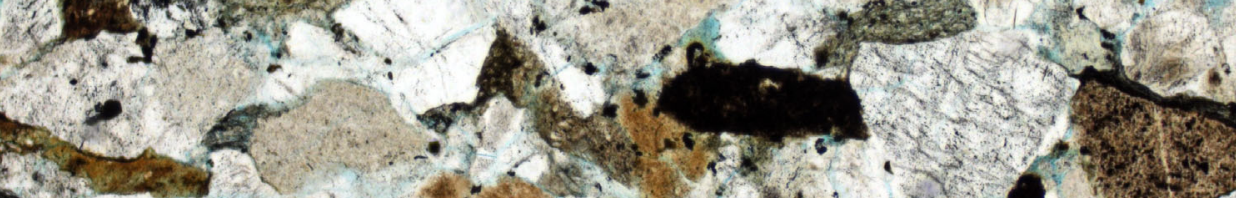
$-2+2 \times 1$ $5=x^{3}+1+1$ - 37.9 .1

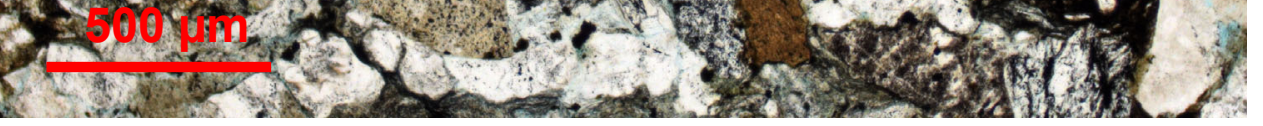
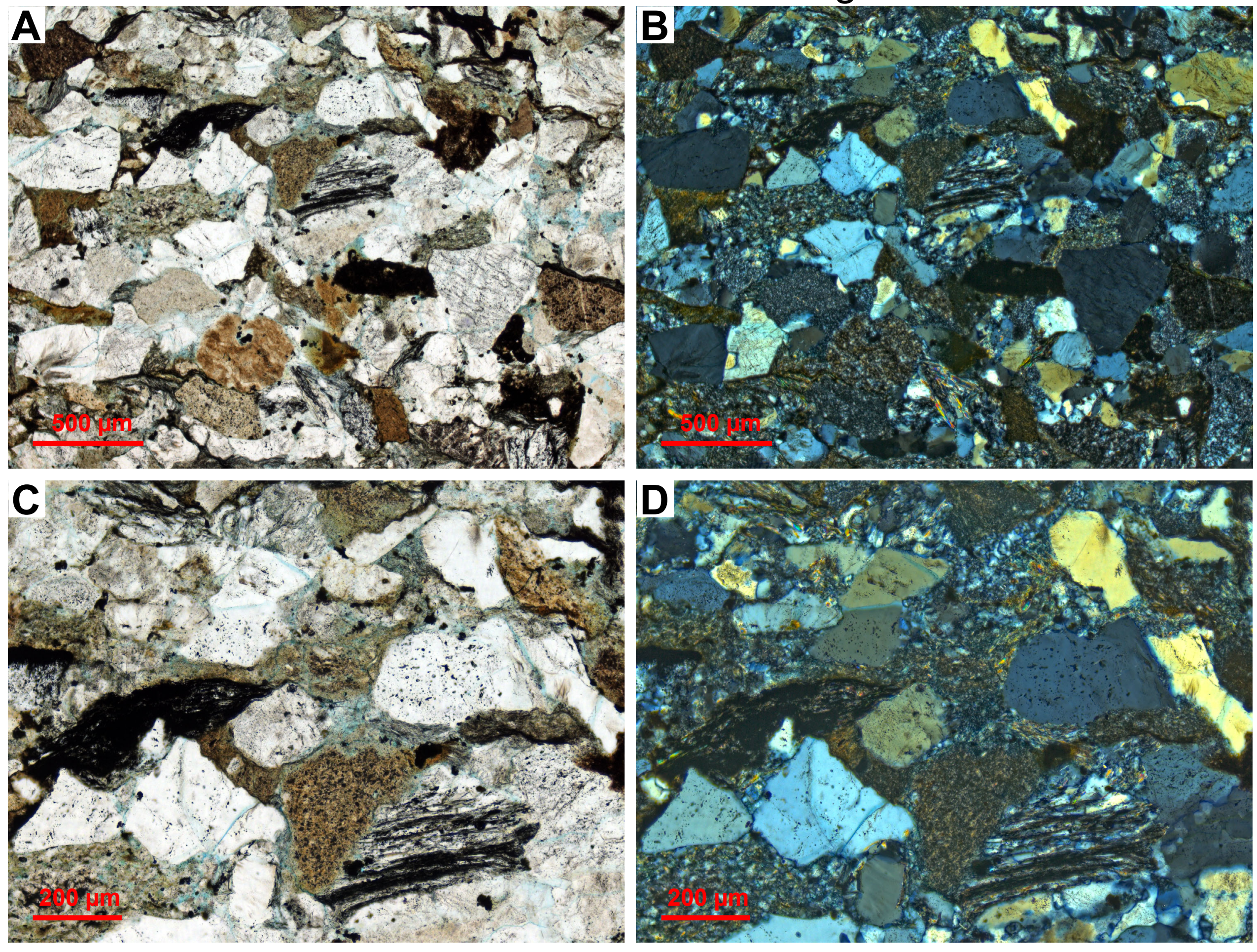


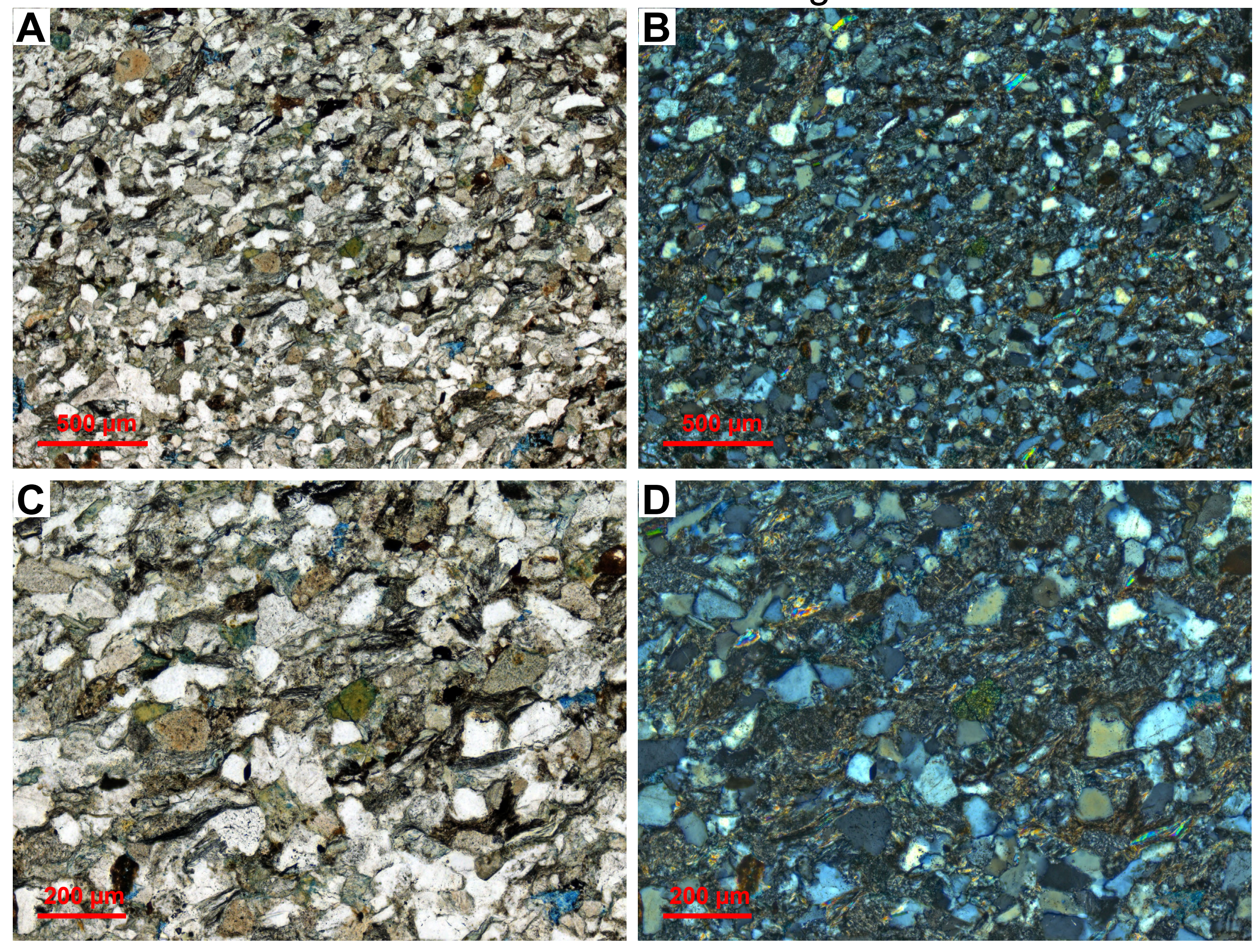


Fig 009 - 18DL002-054.0A

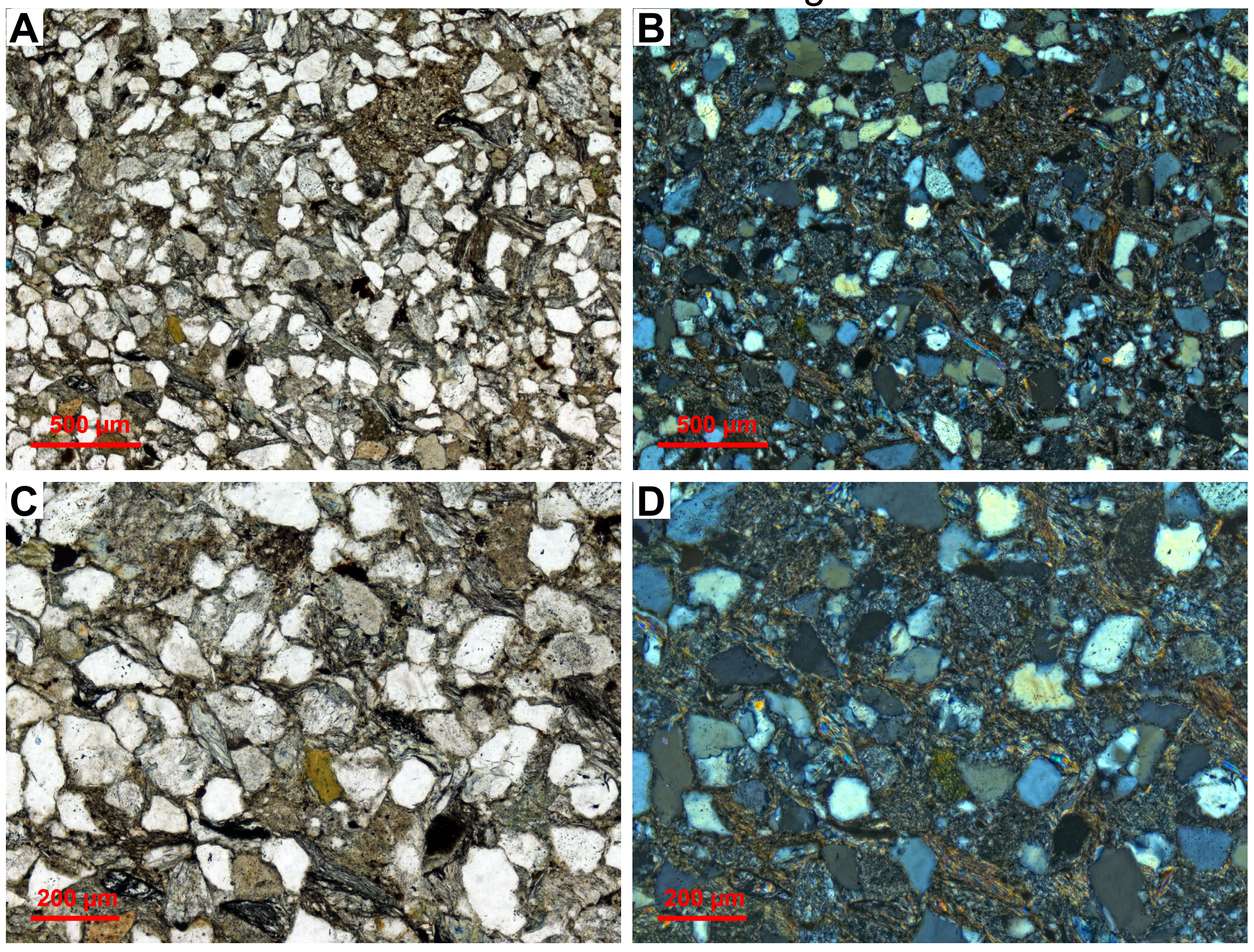


Fig 010 - 18DL002-110.8A

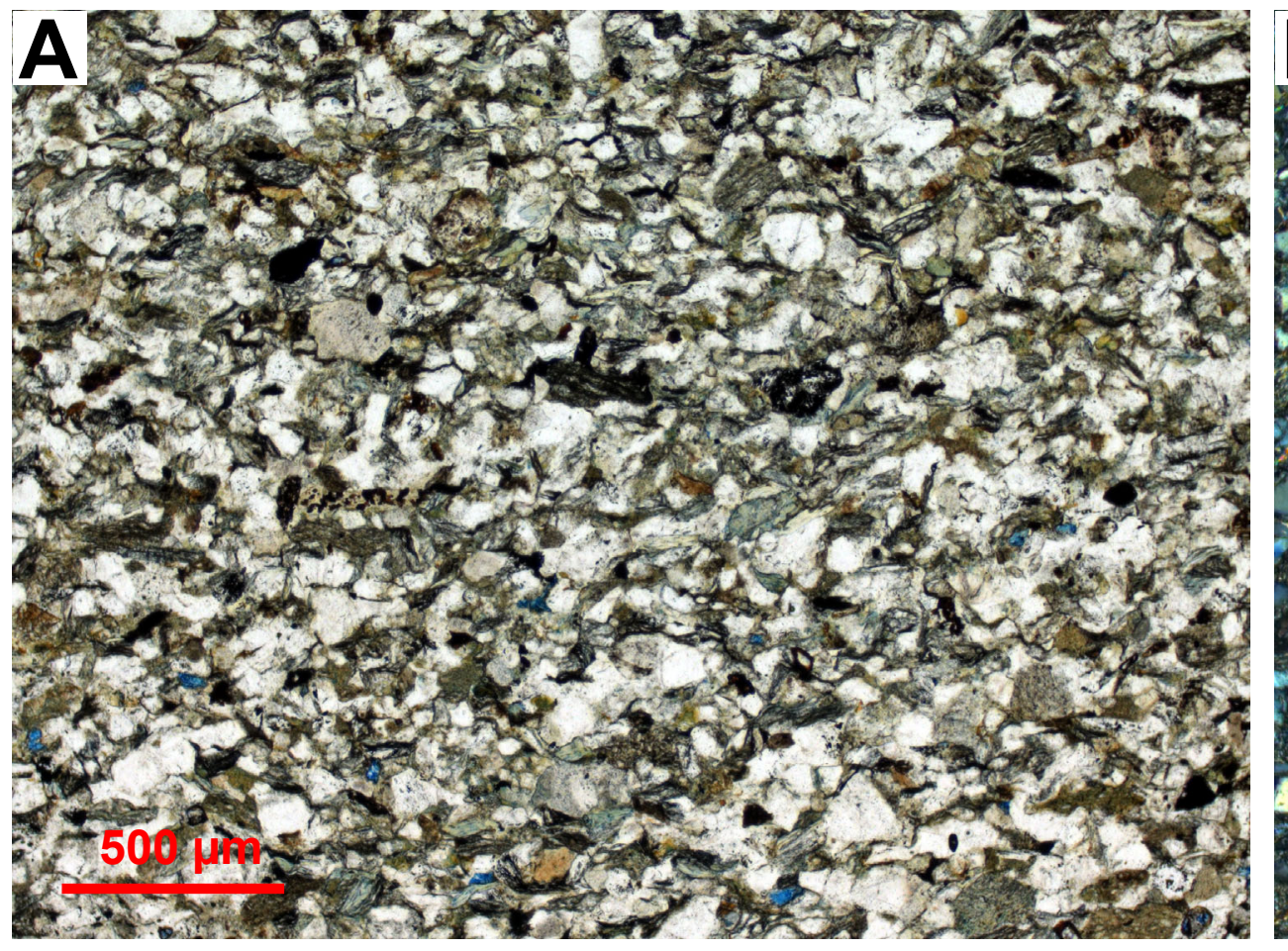

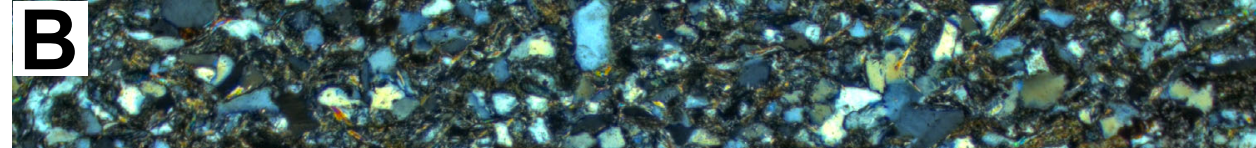

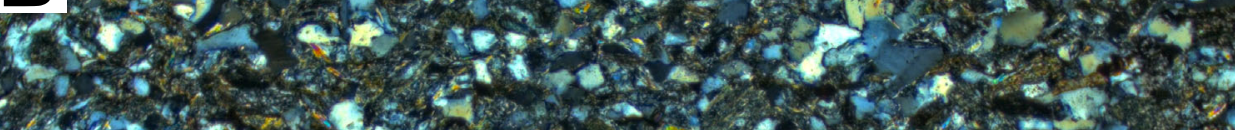

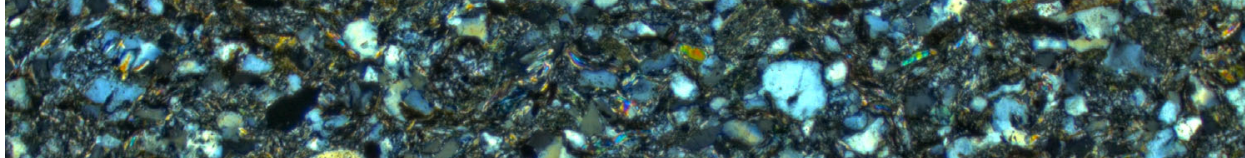

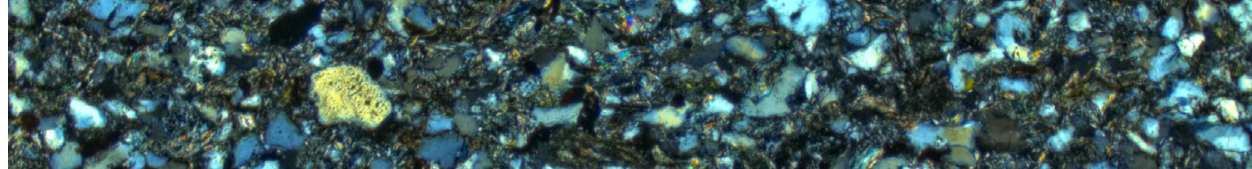

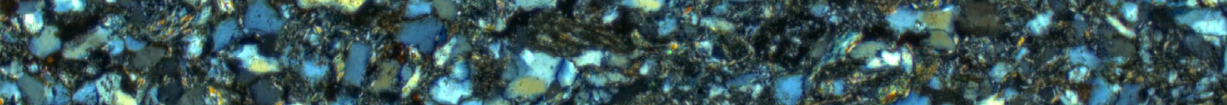

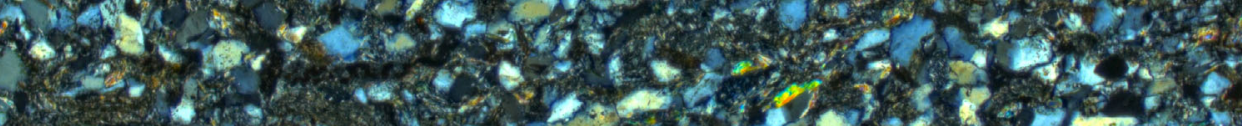

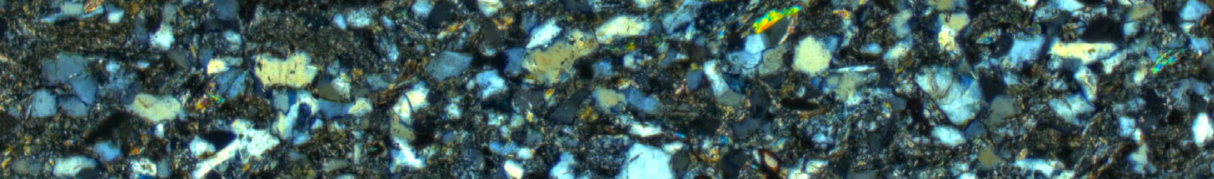

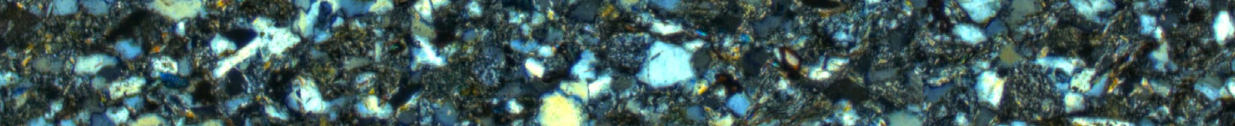

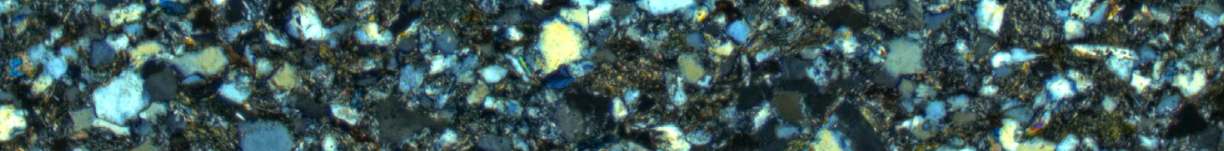

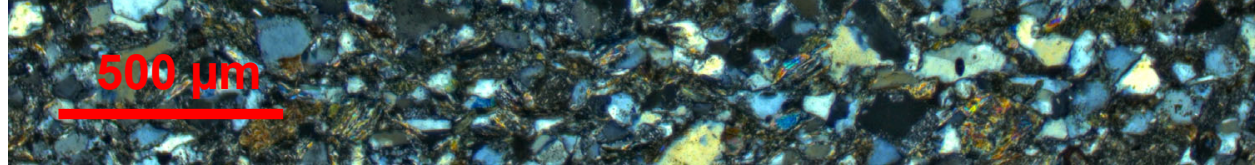

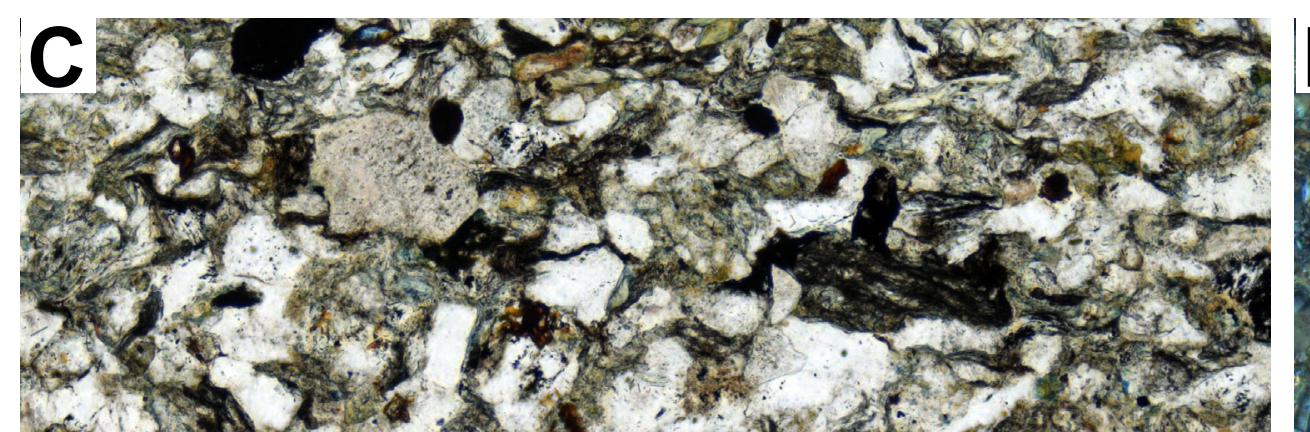

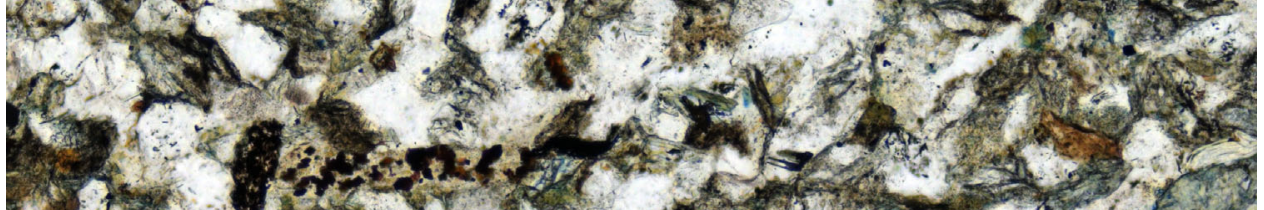

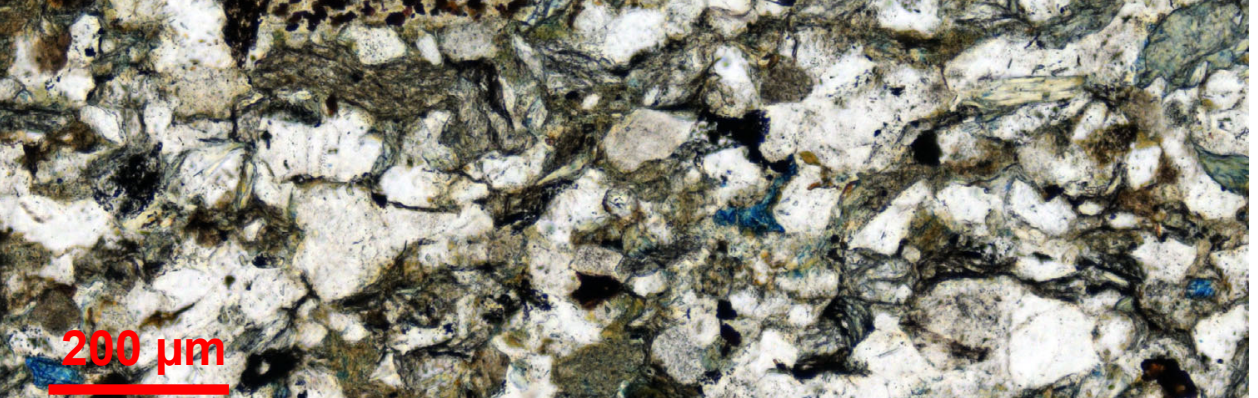

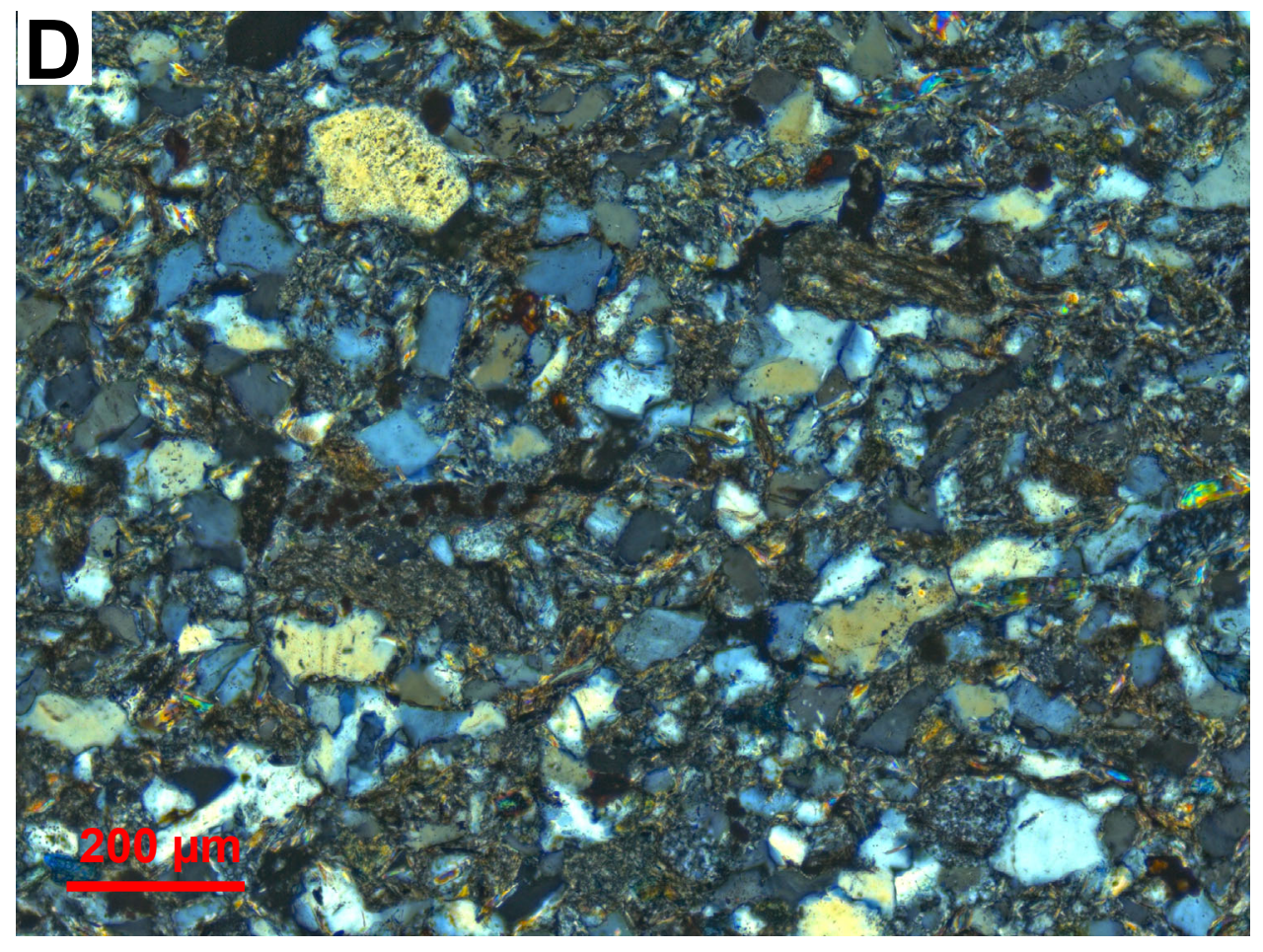


Fig 011 - 18DL002-213.0A
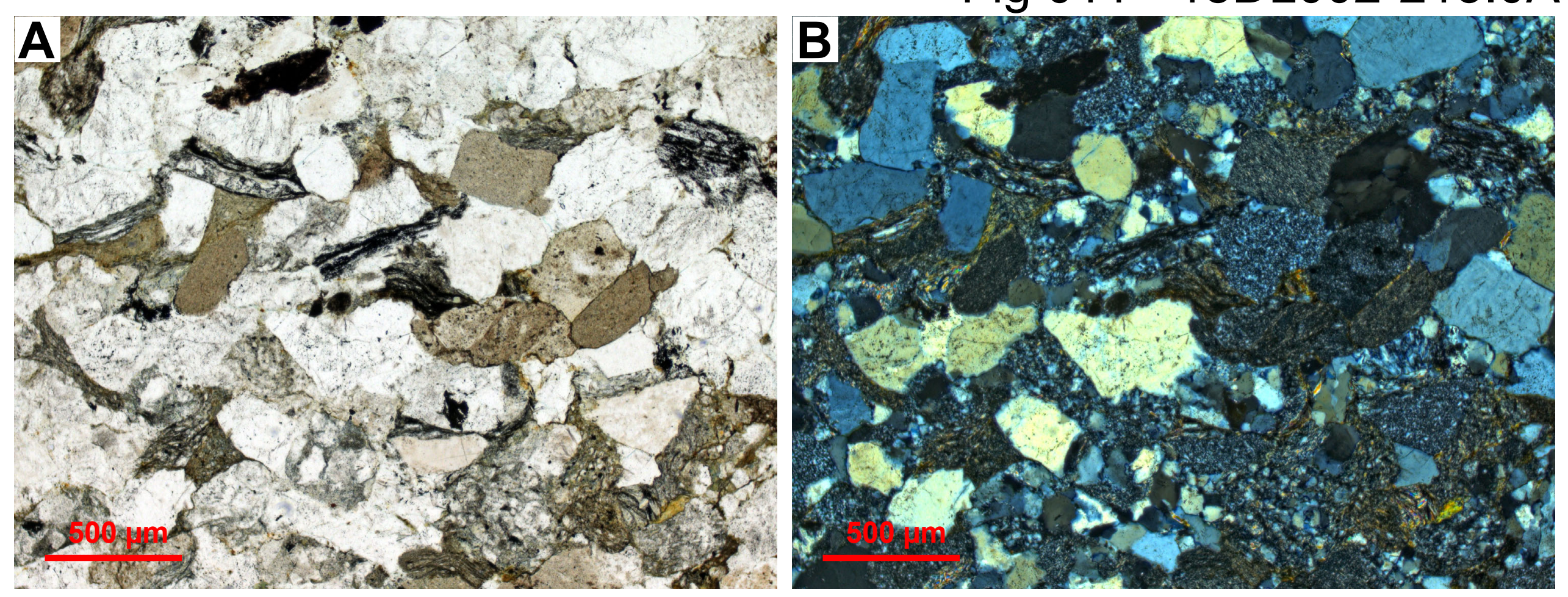

C
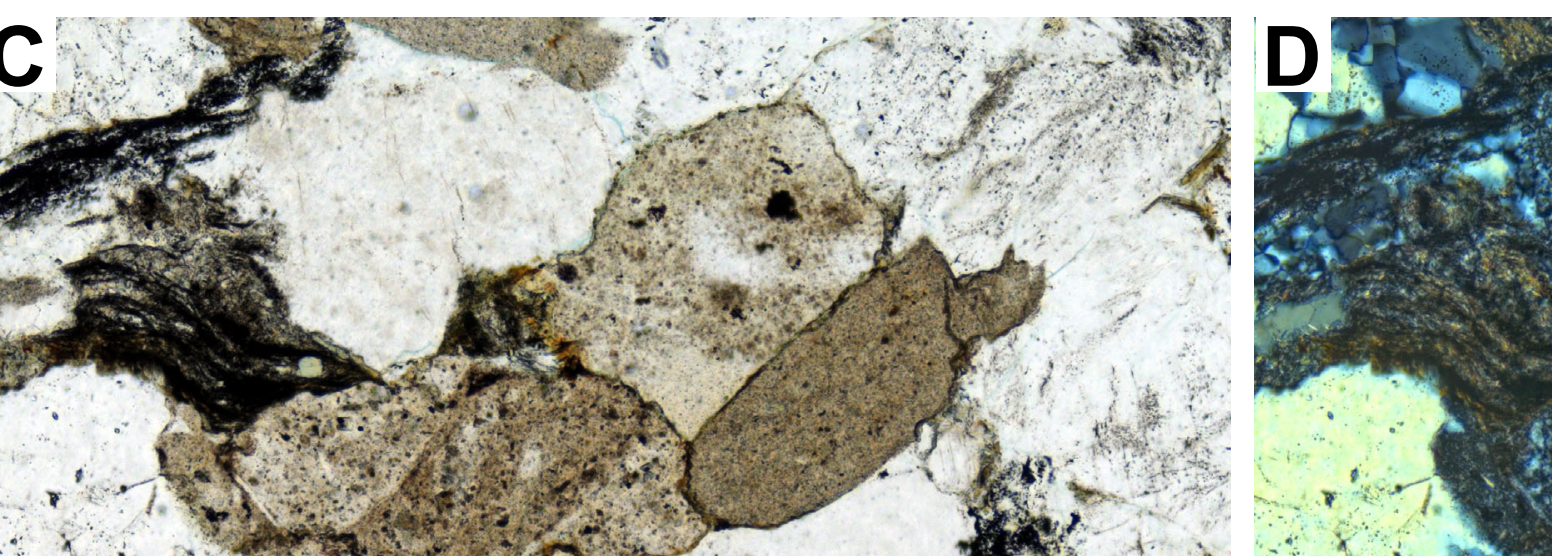

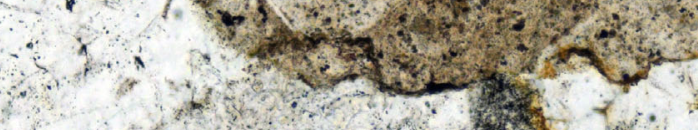
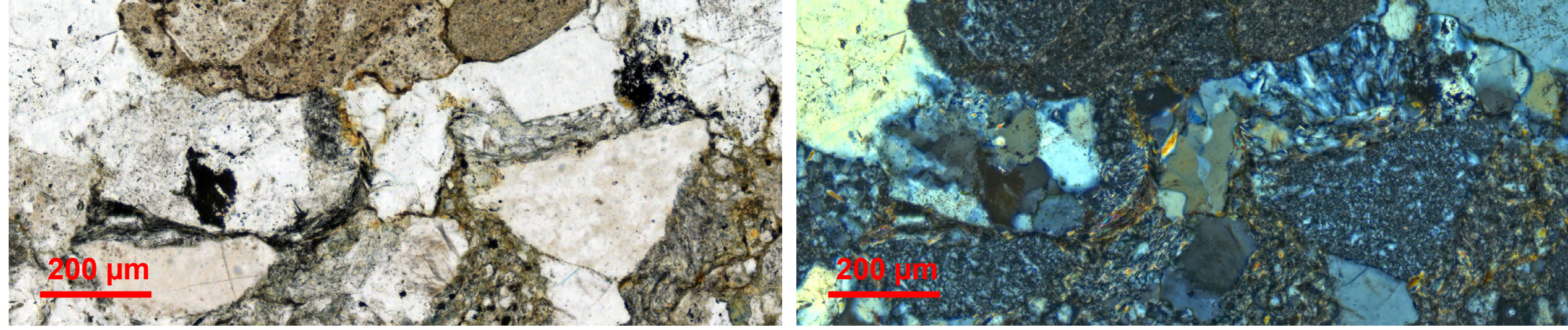
Fig 012 - 18DL002-292.8A

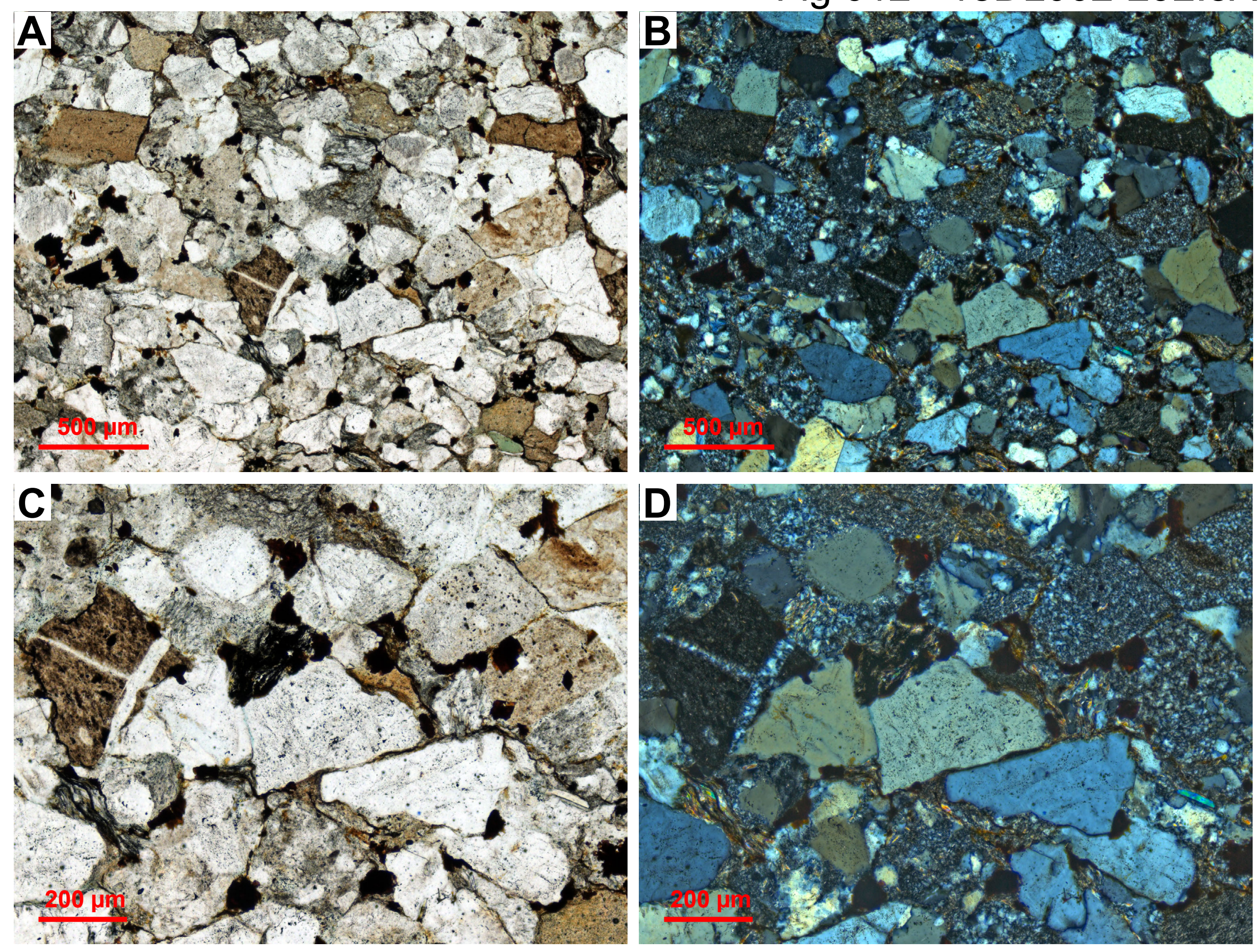


Fig 013 - 19DL002-002.0A
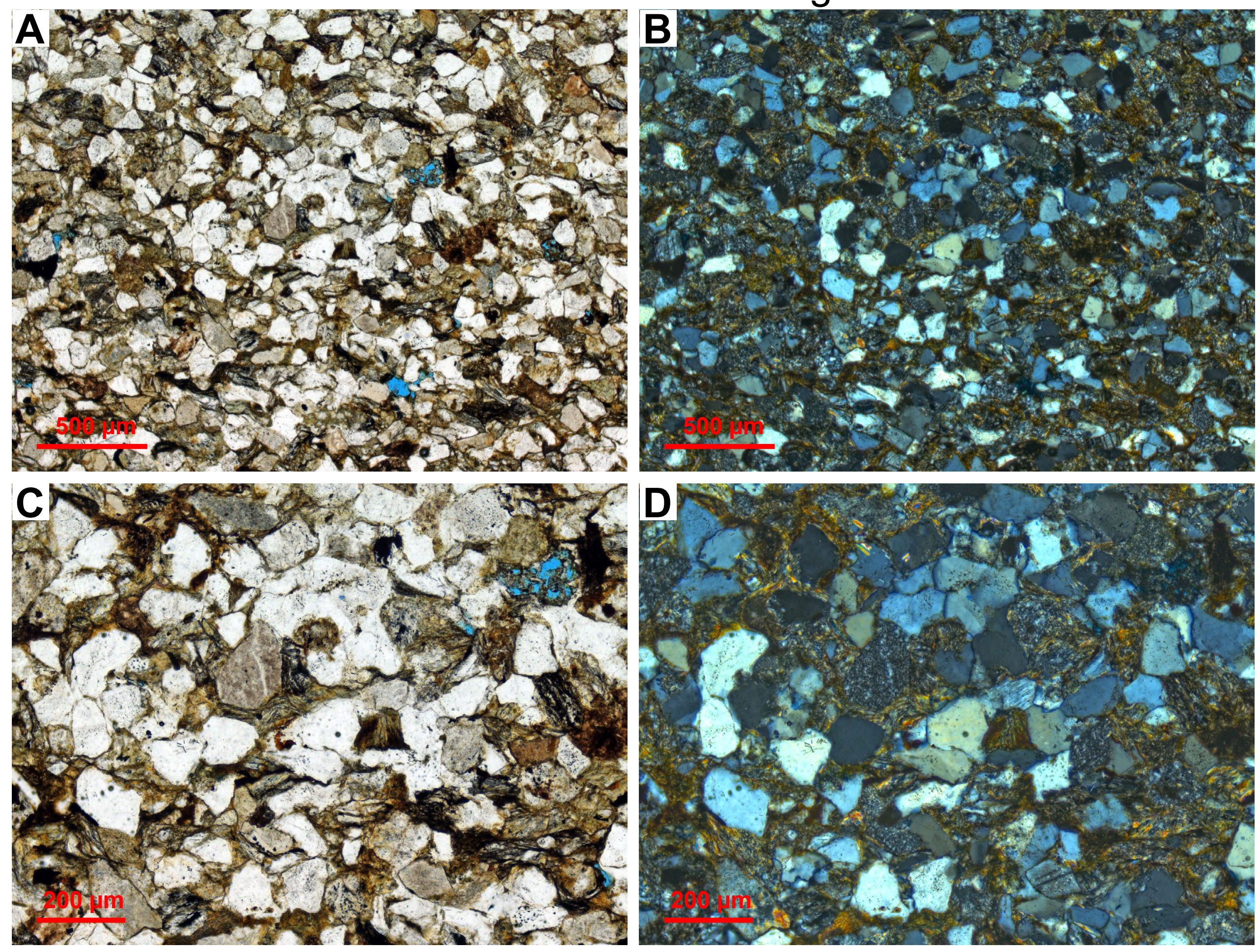
Fig 014 - 01DL027-003.0

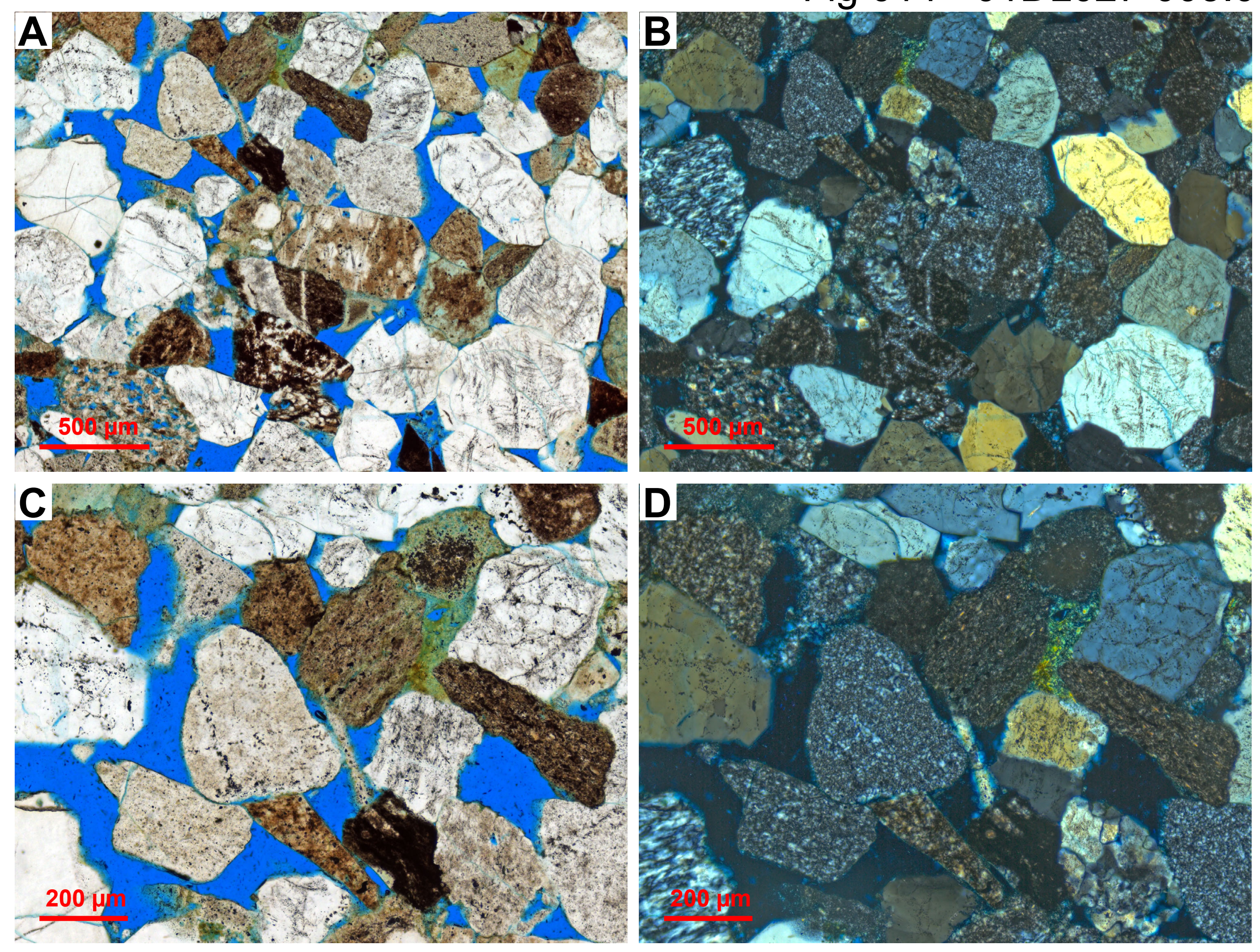


Fig 015 - 01DL027-011.0

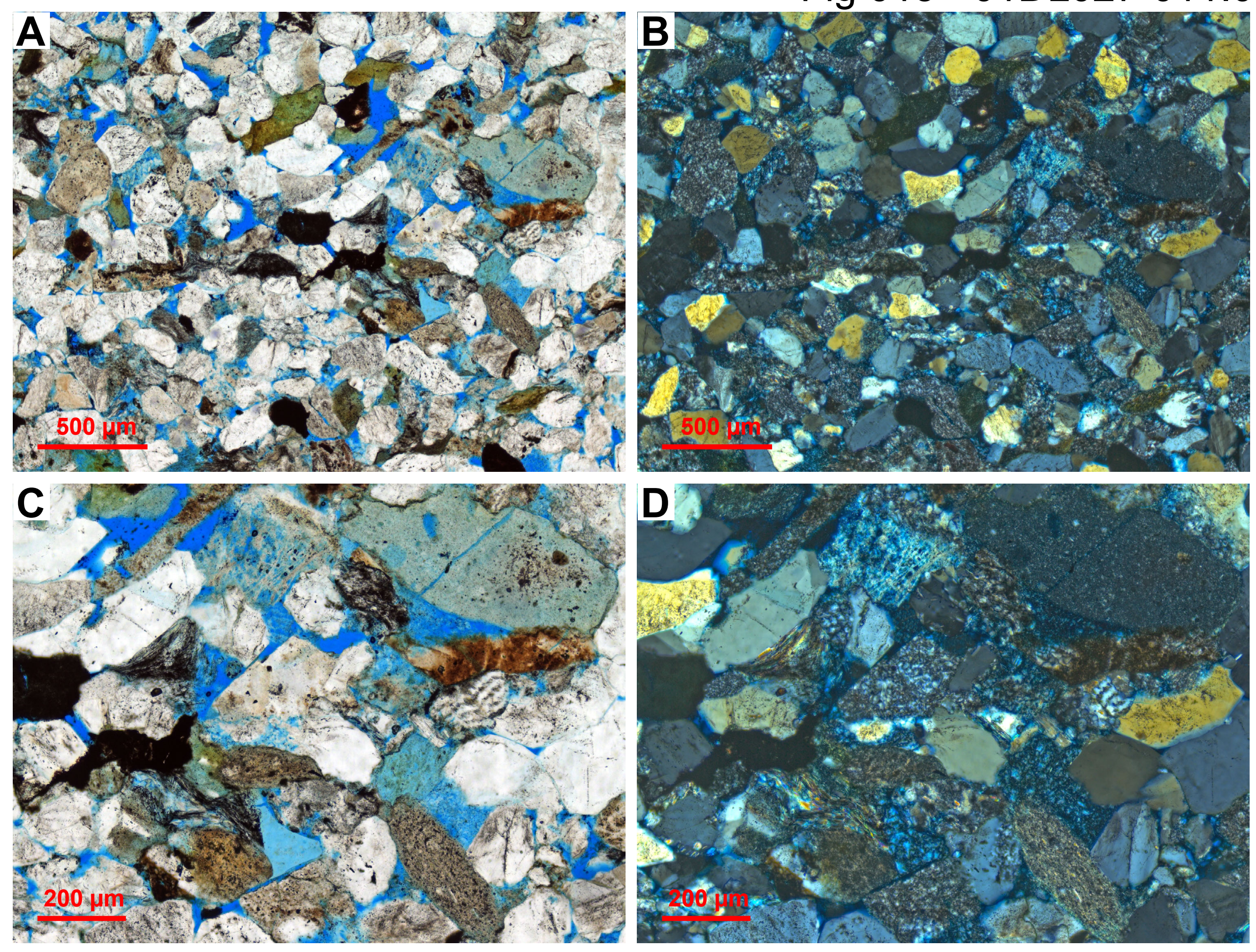


Fig 016 - 01DL027-028.0
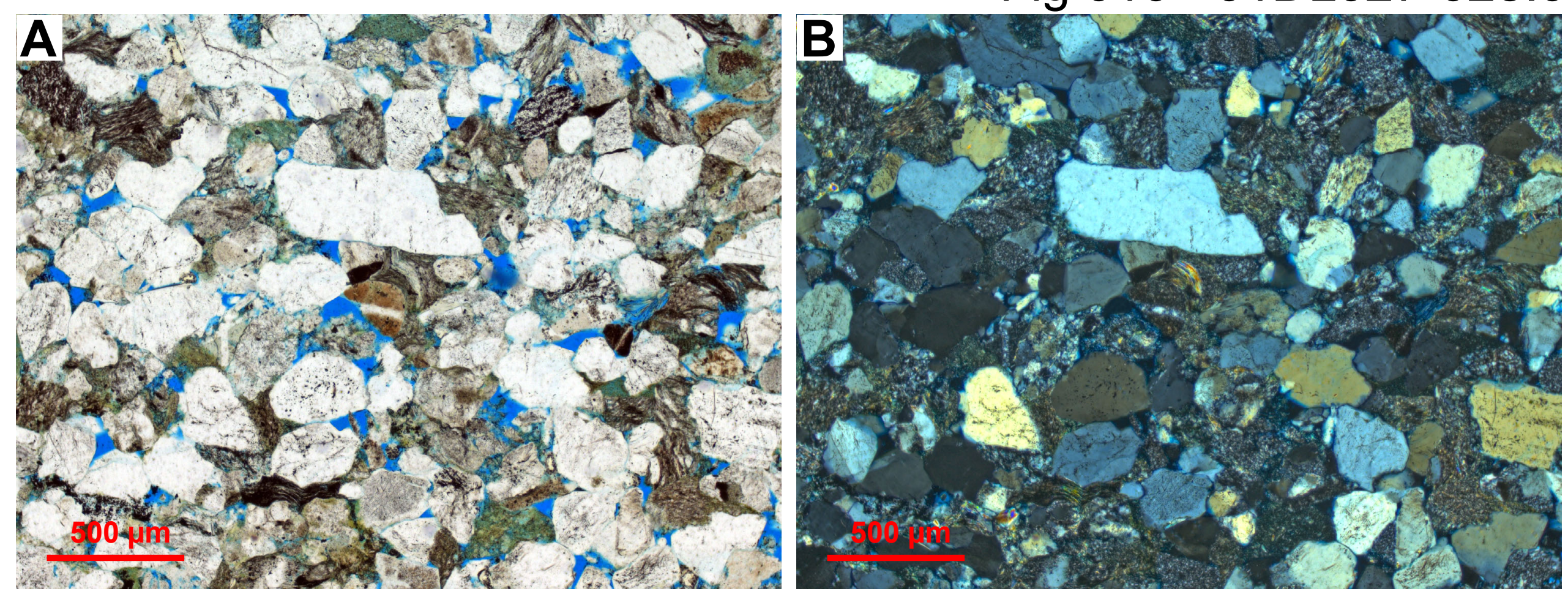

C
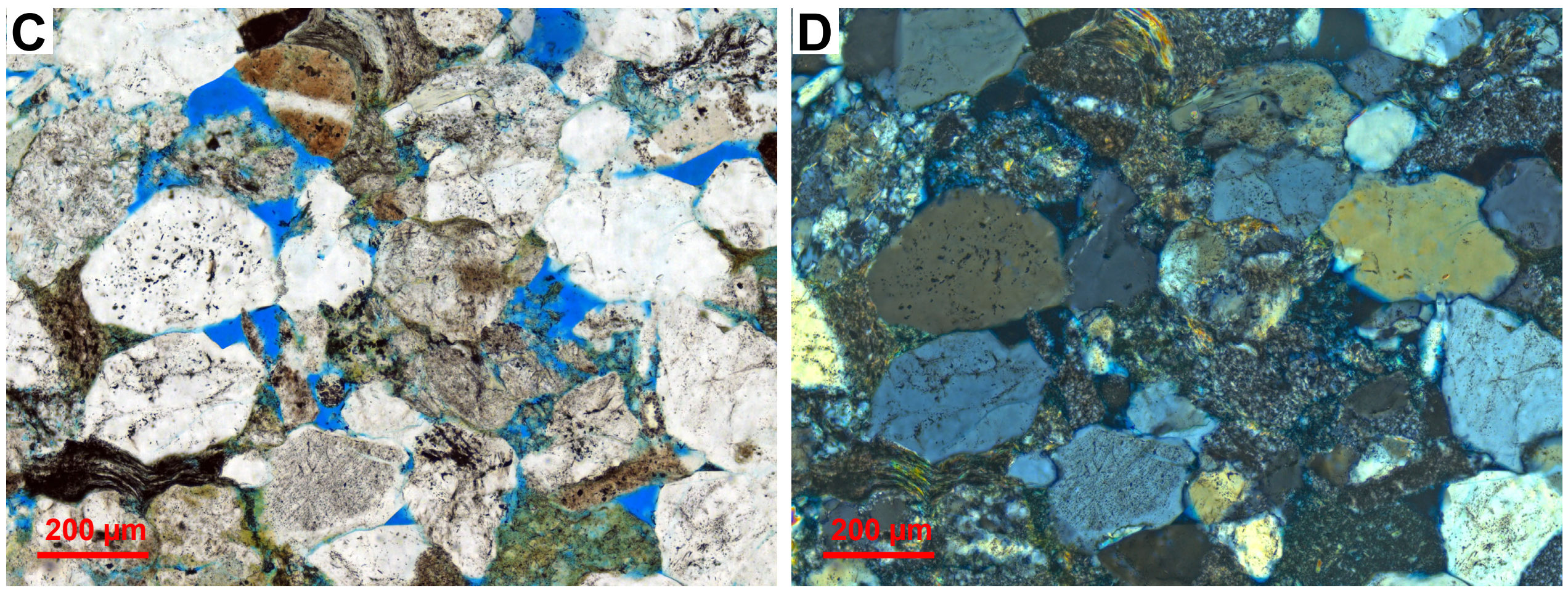
Fig 017 - 99DL047B-000.5
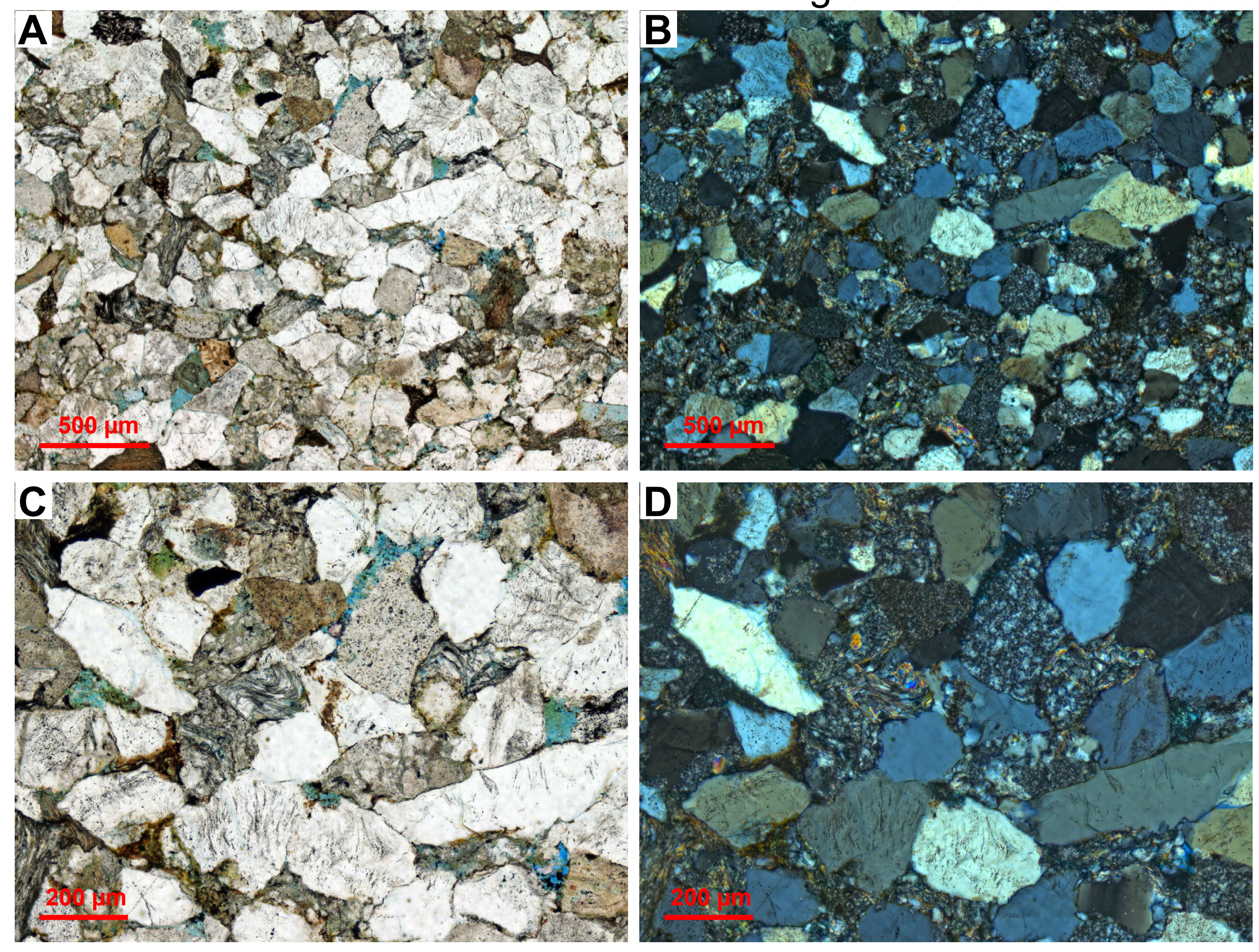
Fig 018 - 99DL047B-071.0

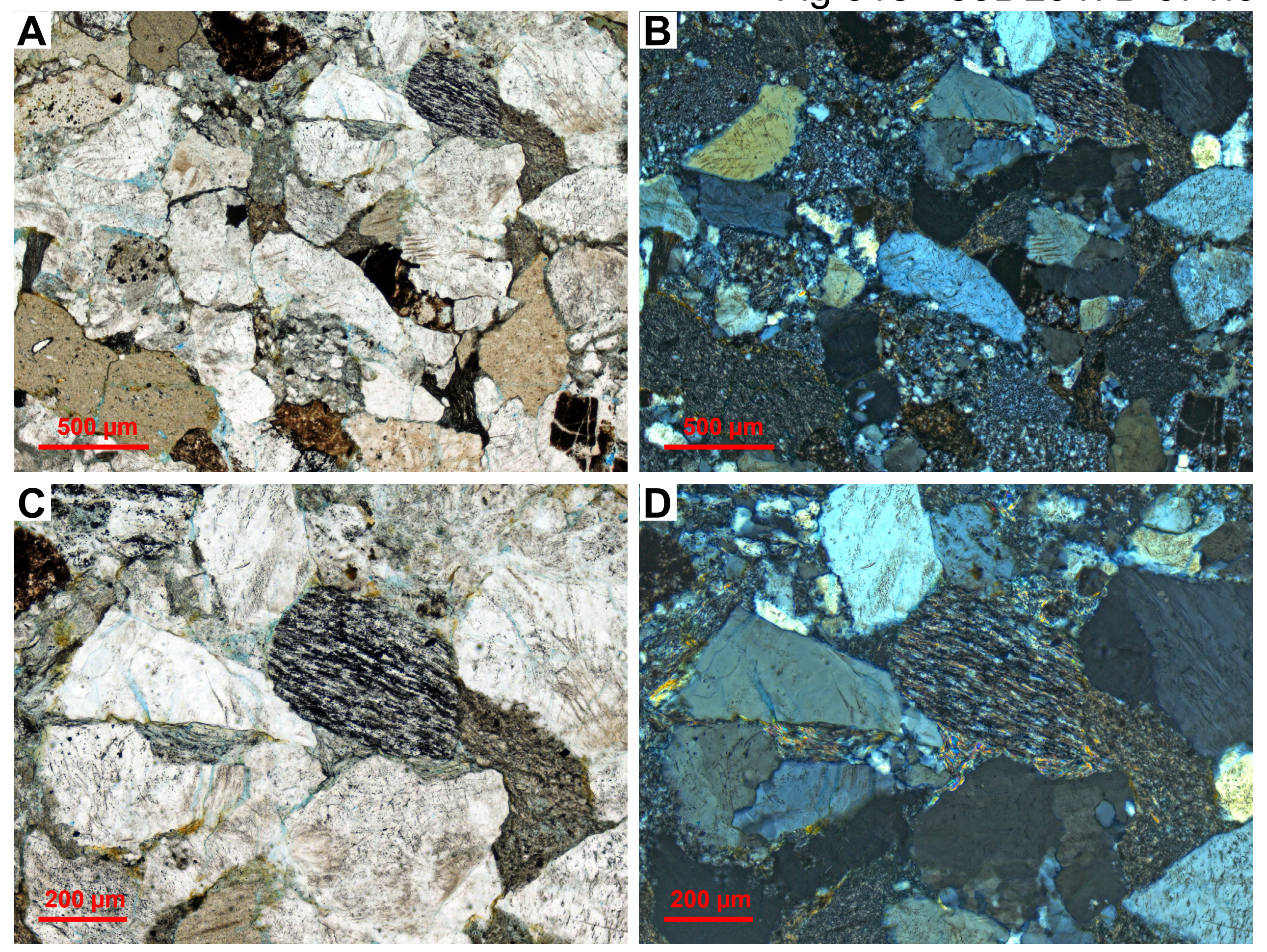


Fig 019 - 99DL047B-073.0

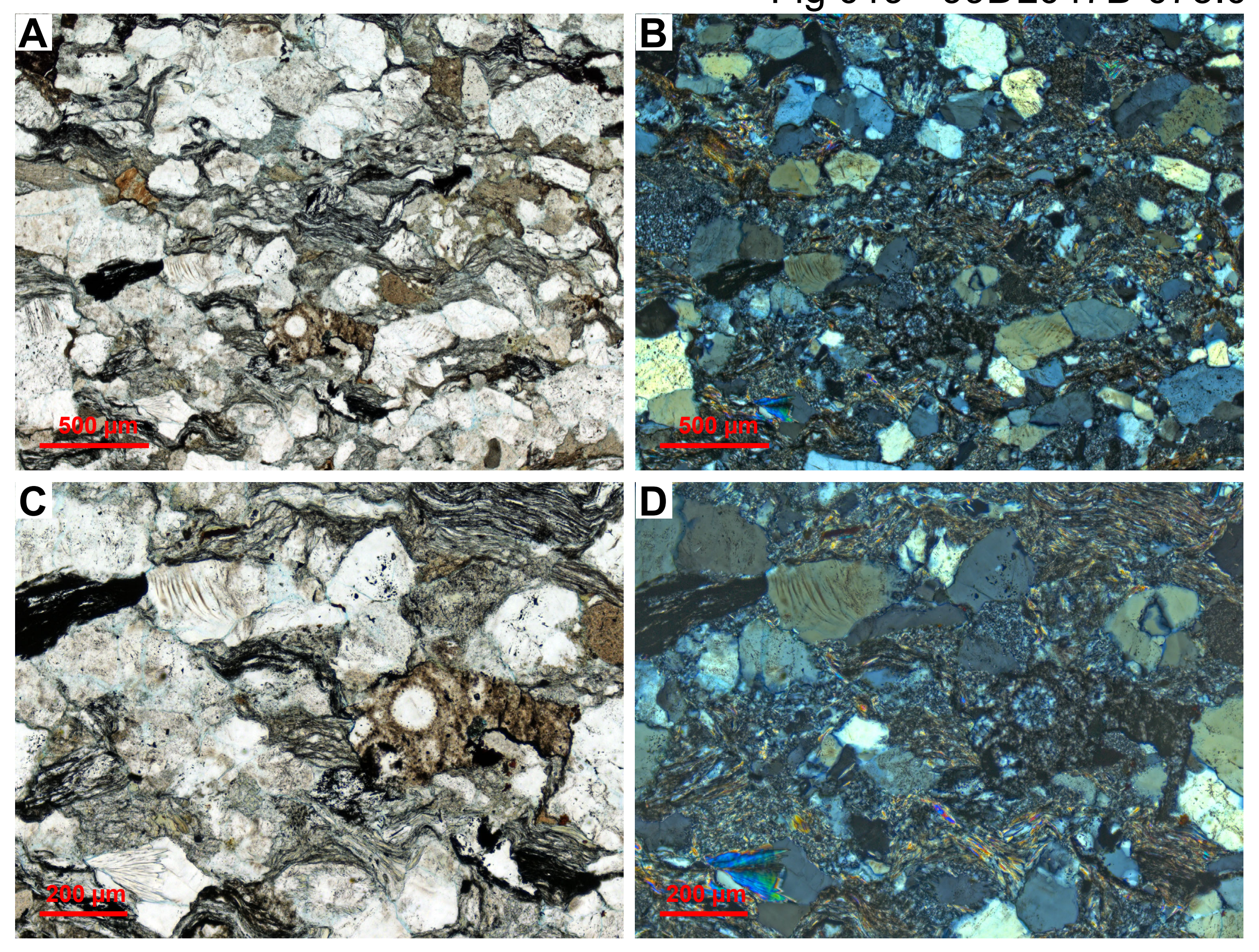


Fig 020 - 99DL047C-001.3
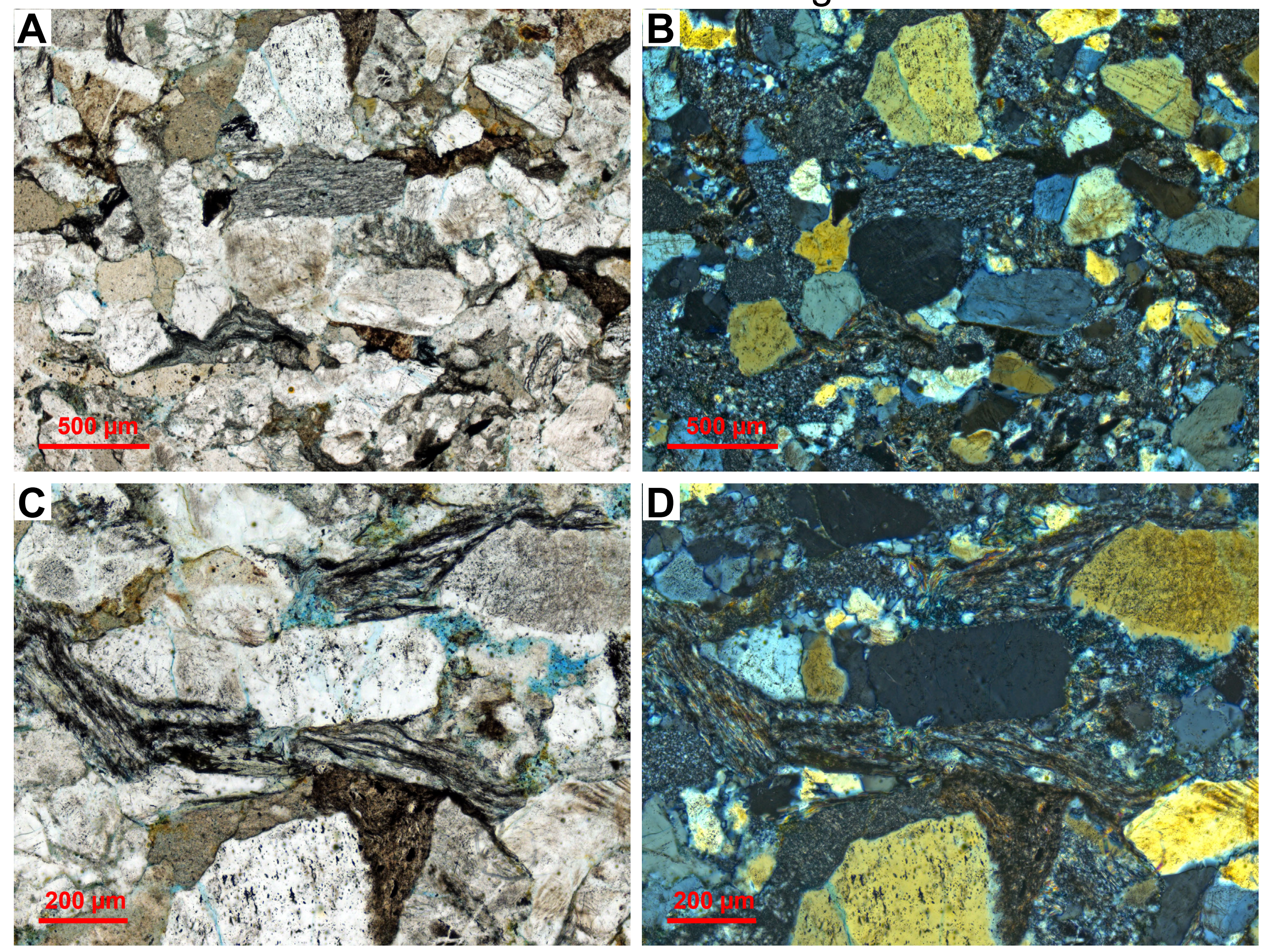
Fig 021 - 99DL047C-010.3

A.

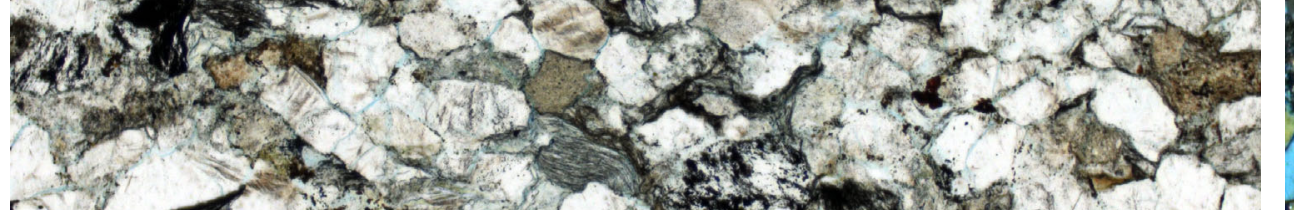
$29-1501$

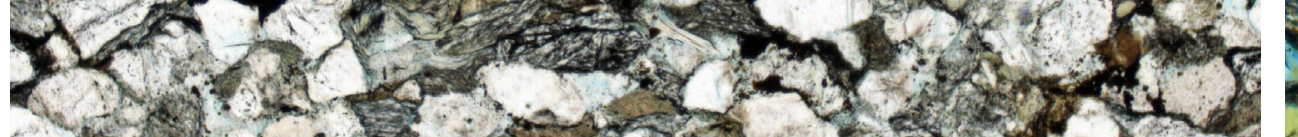
bactorid?

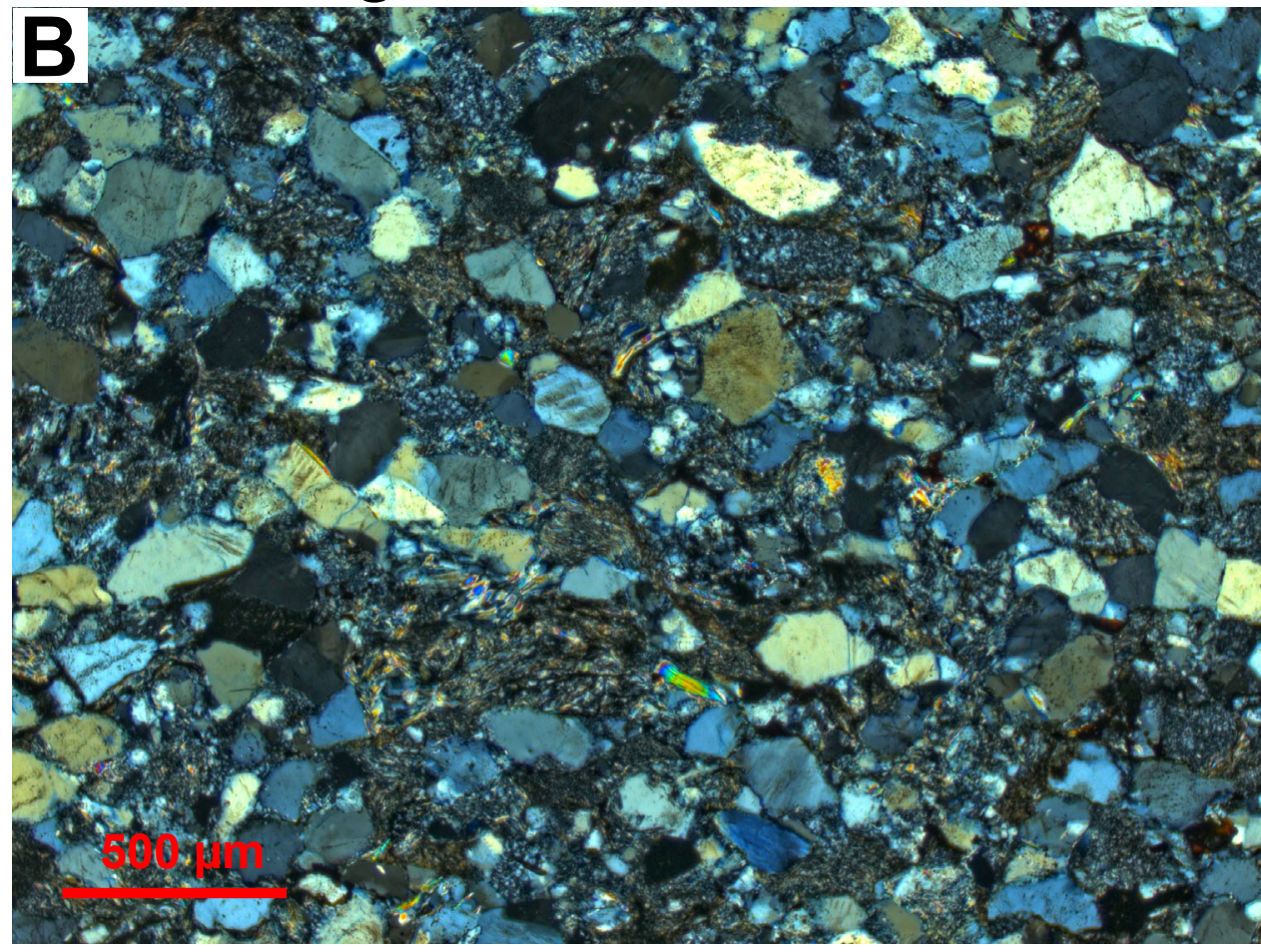

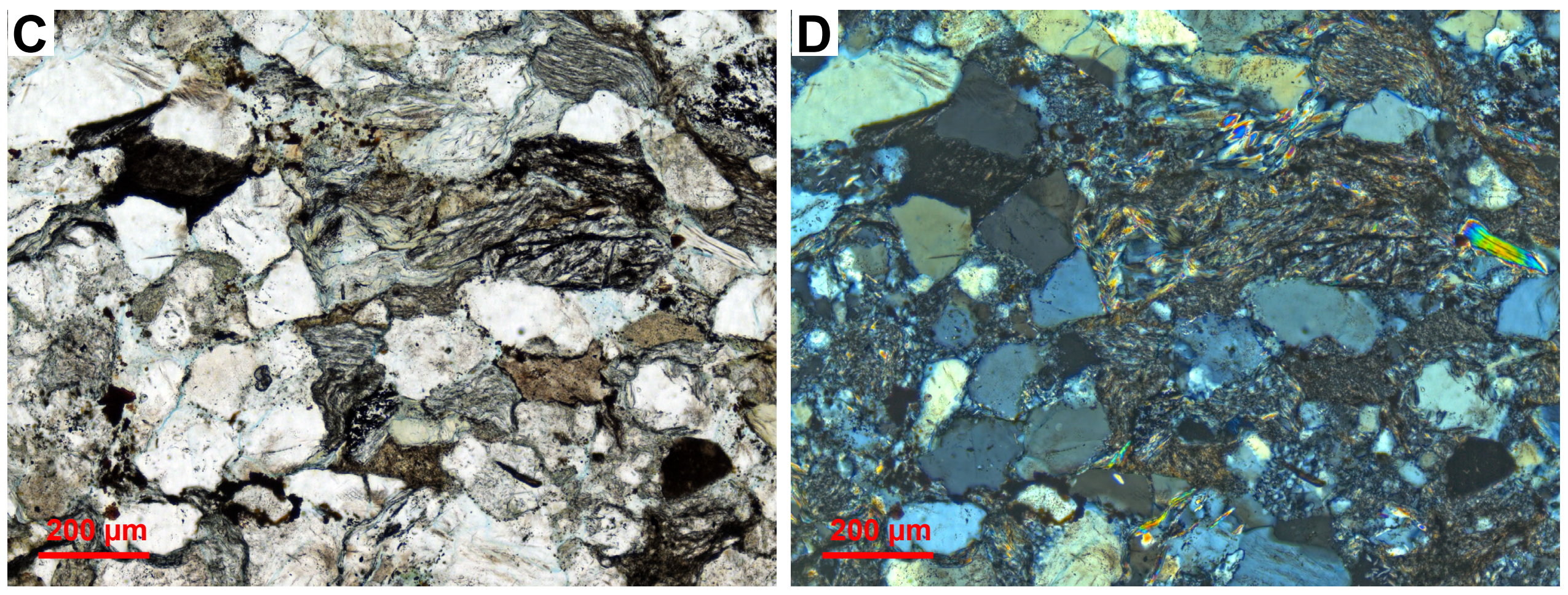


Fig 022 - 99DL047C-016.2
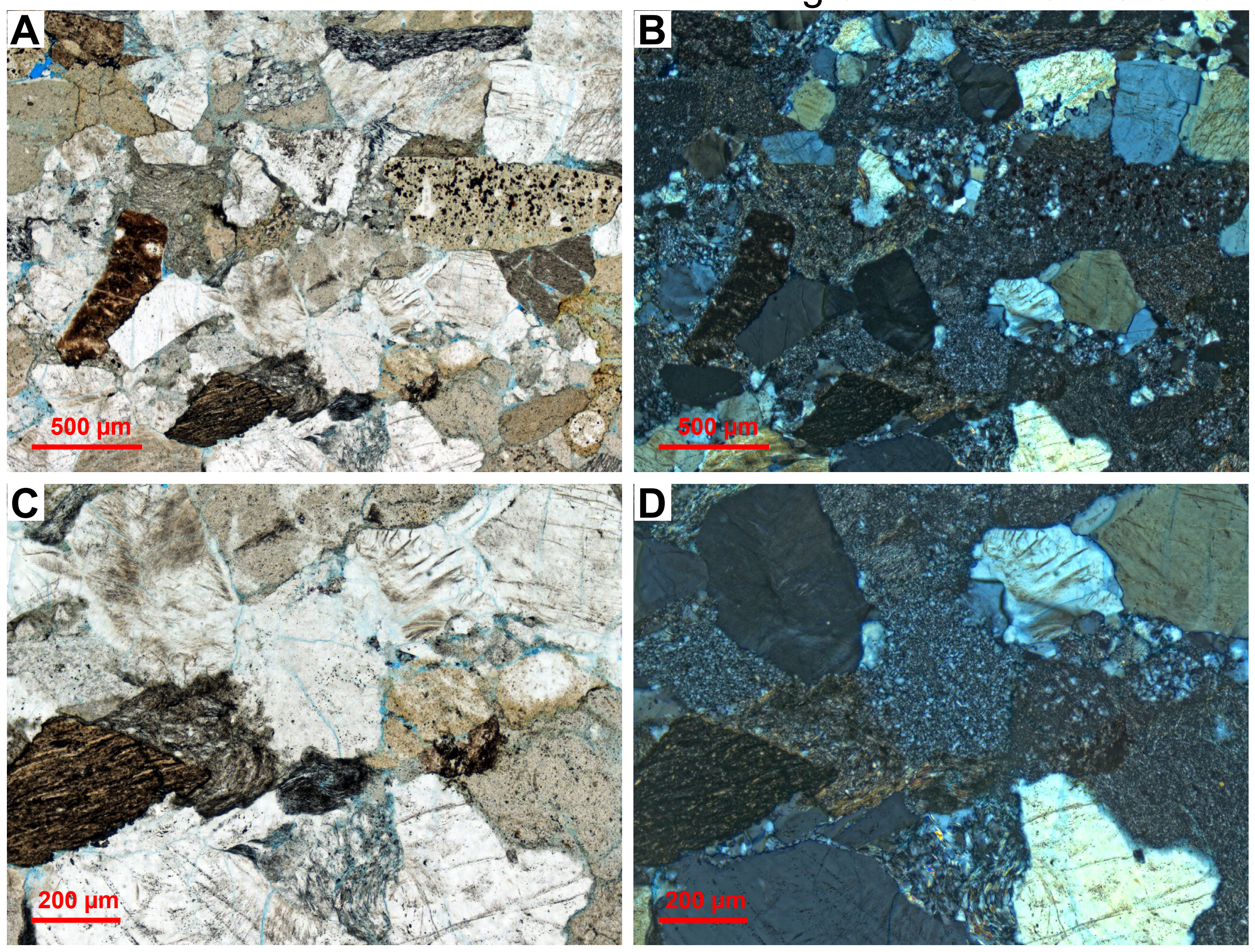
Fig 023 - 99DL047C-017.9

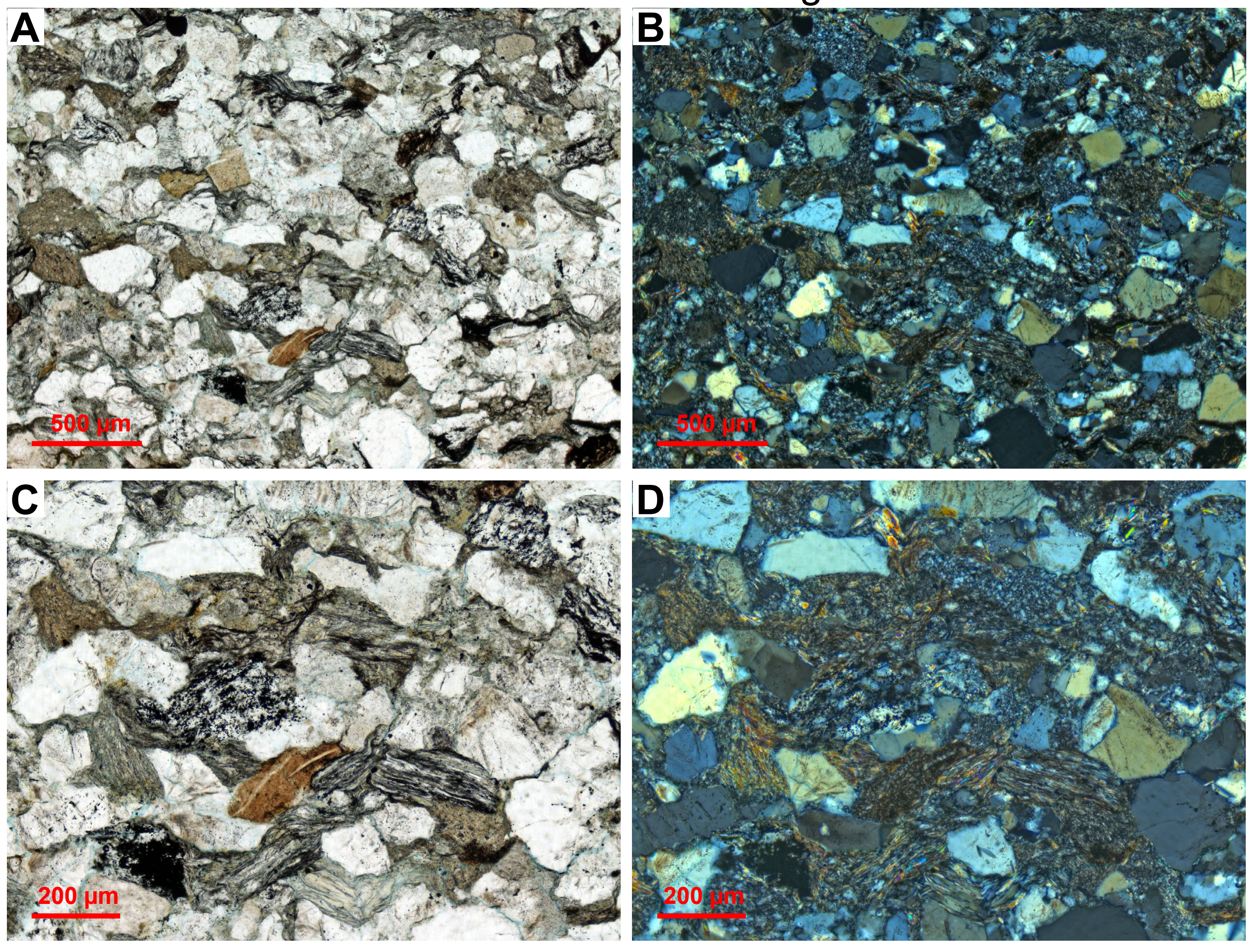


Fig 024 - 99DL047C-026.2
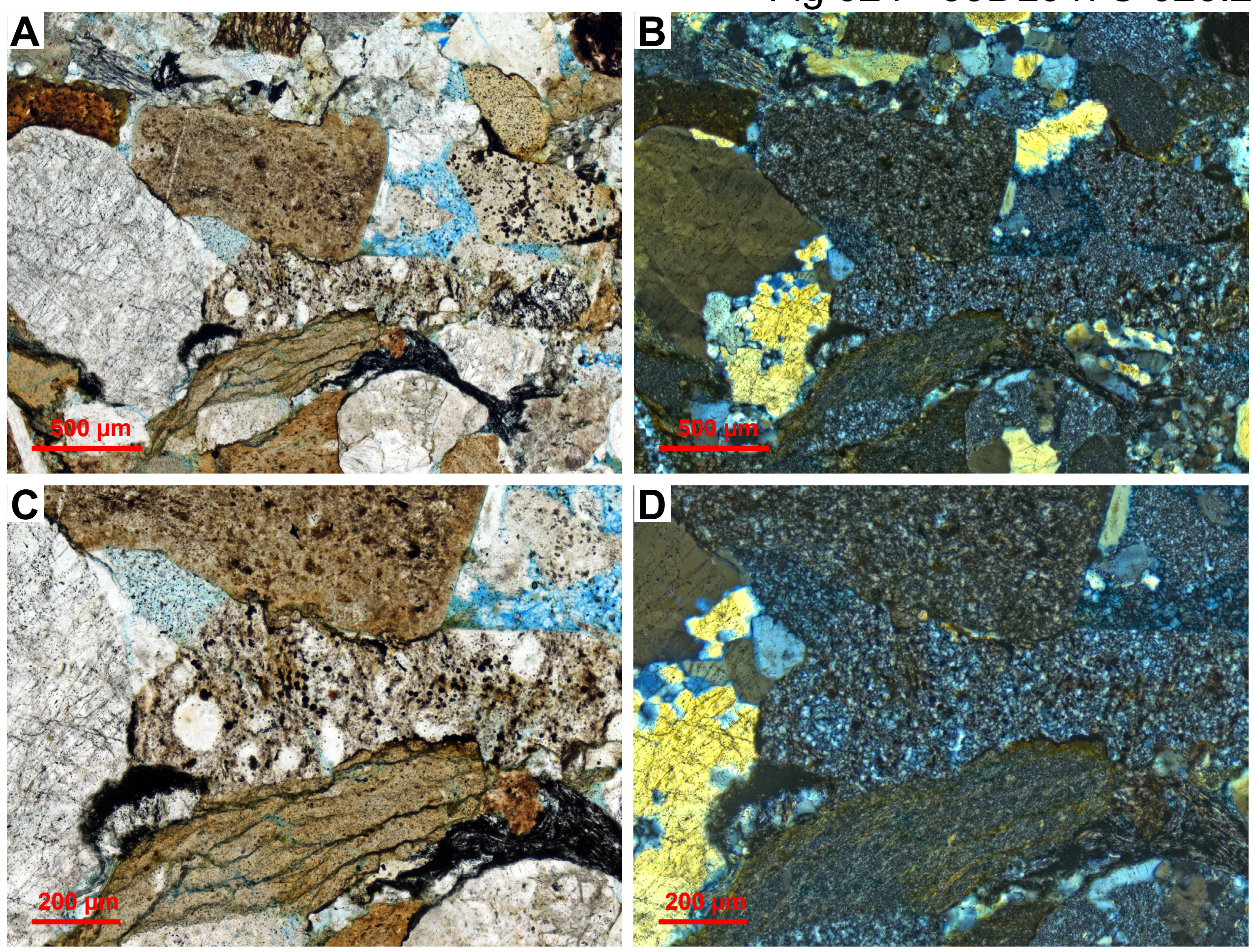
Fig 025 - 99DL047D-002.0

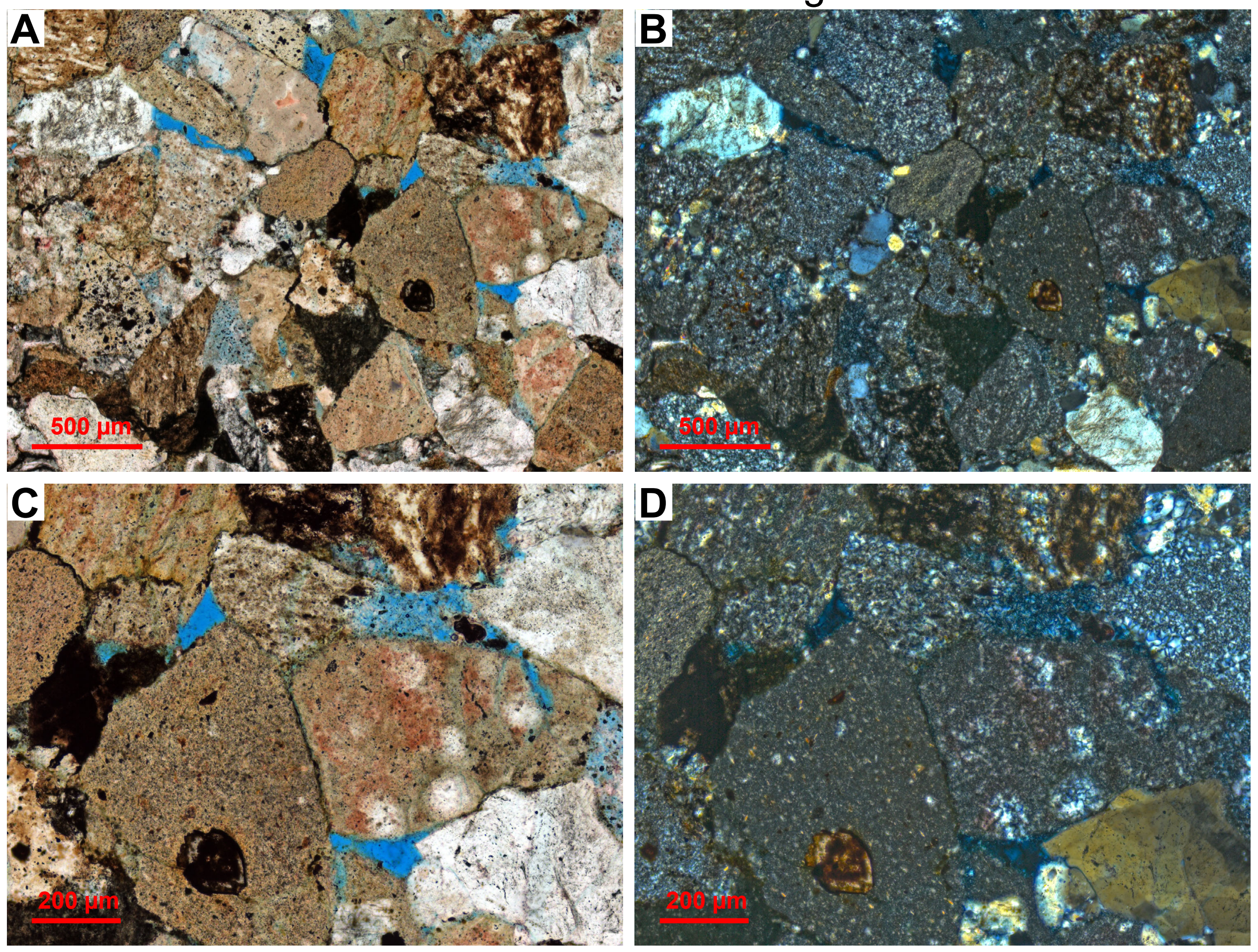


Fig 026 - 99DL047D-007.4
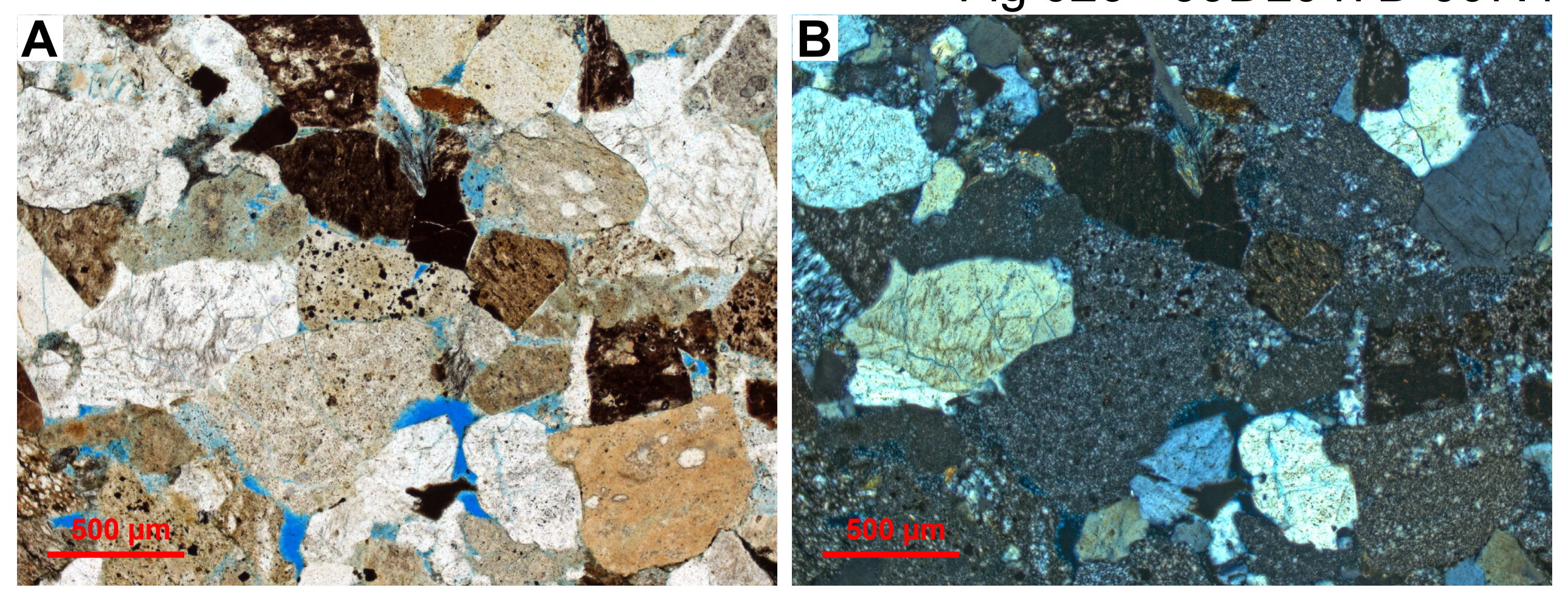

c 40,1001
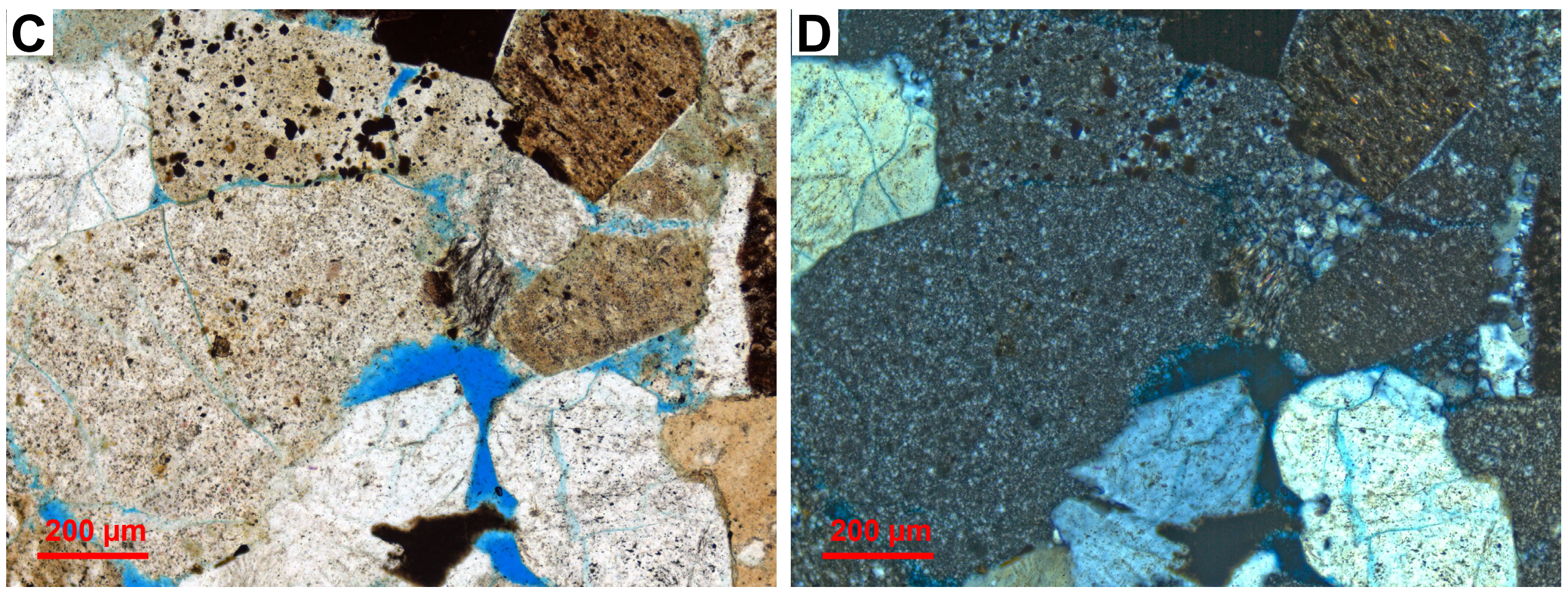
Fig 027 - 99DL047E-009.0

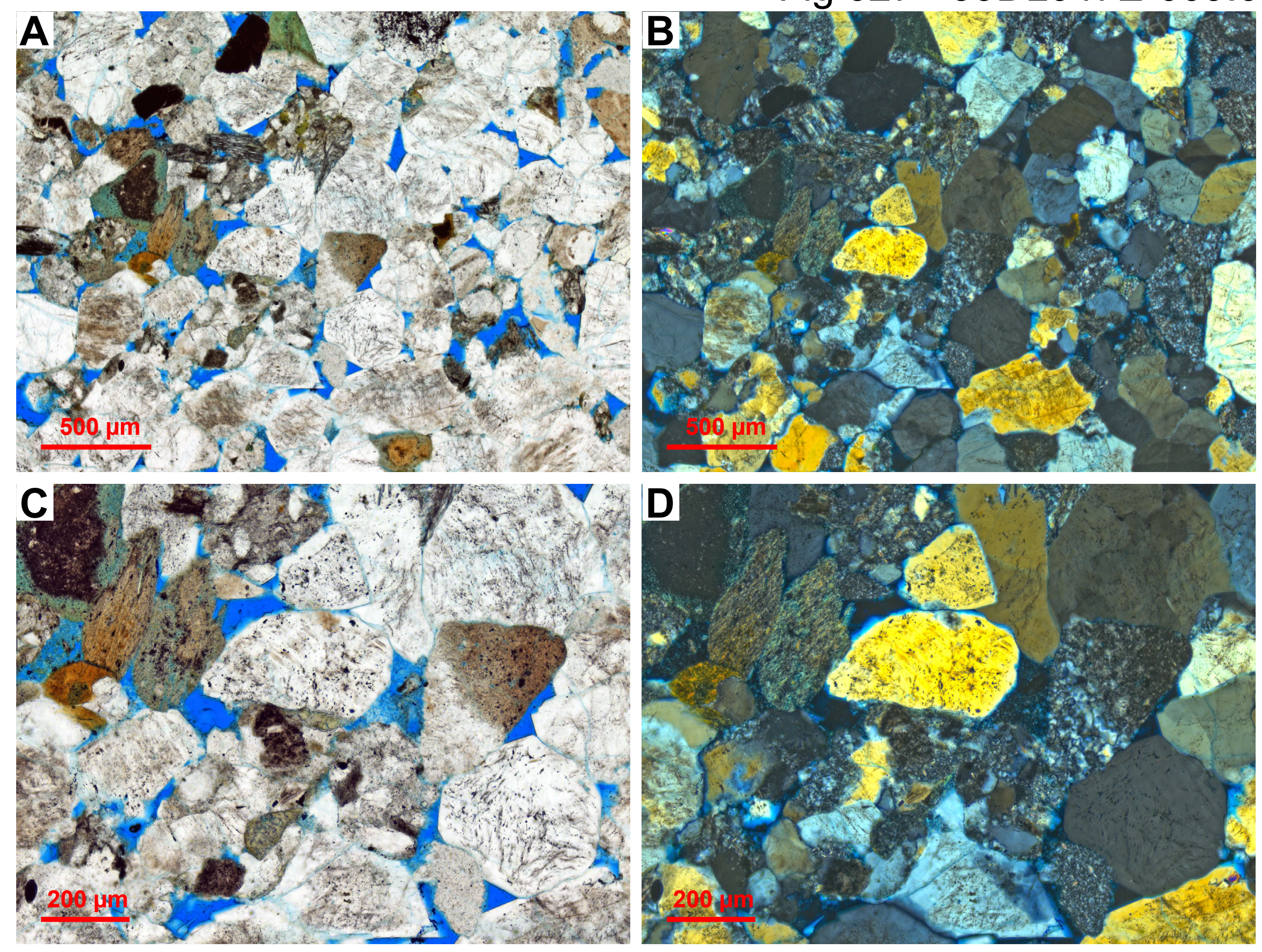


Fig 028 - 00DL024-001.1
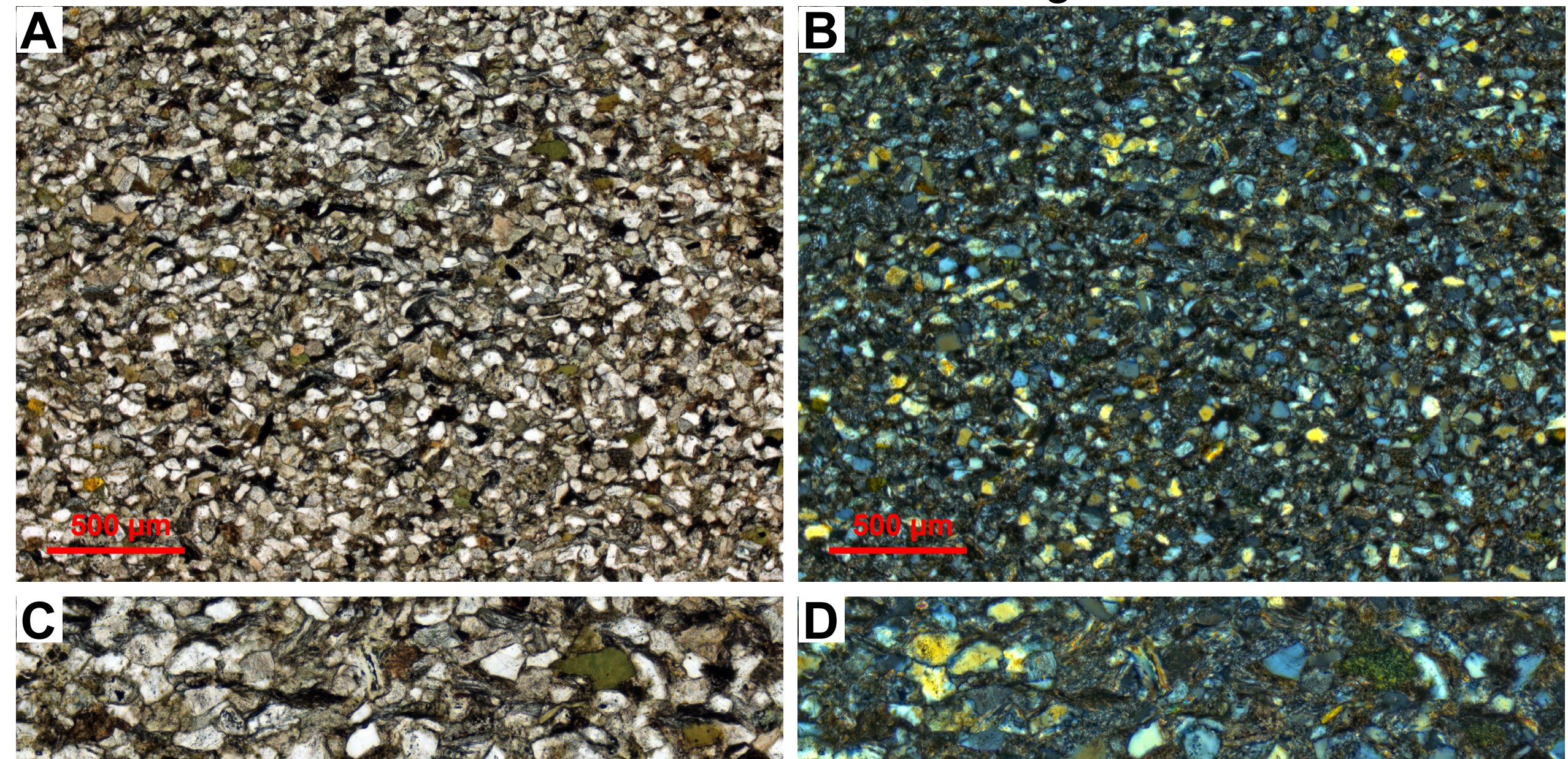

1. 4727501289 c5.

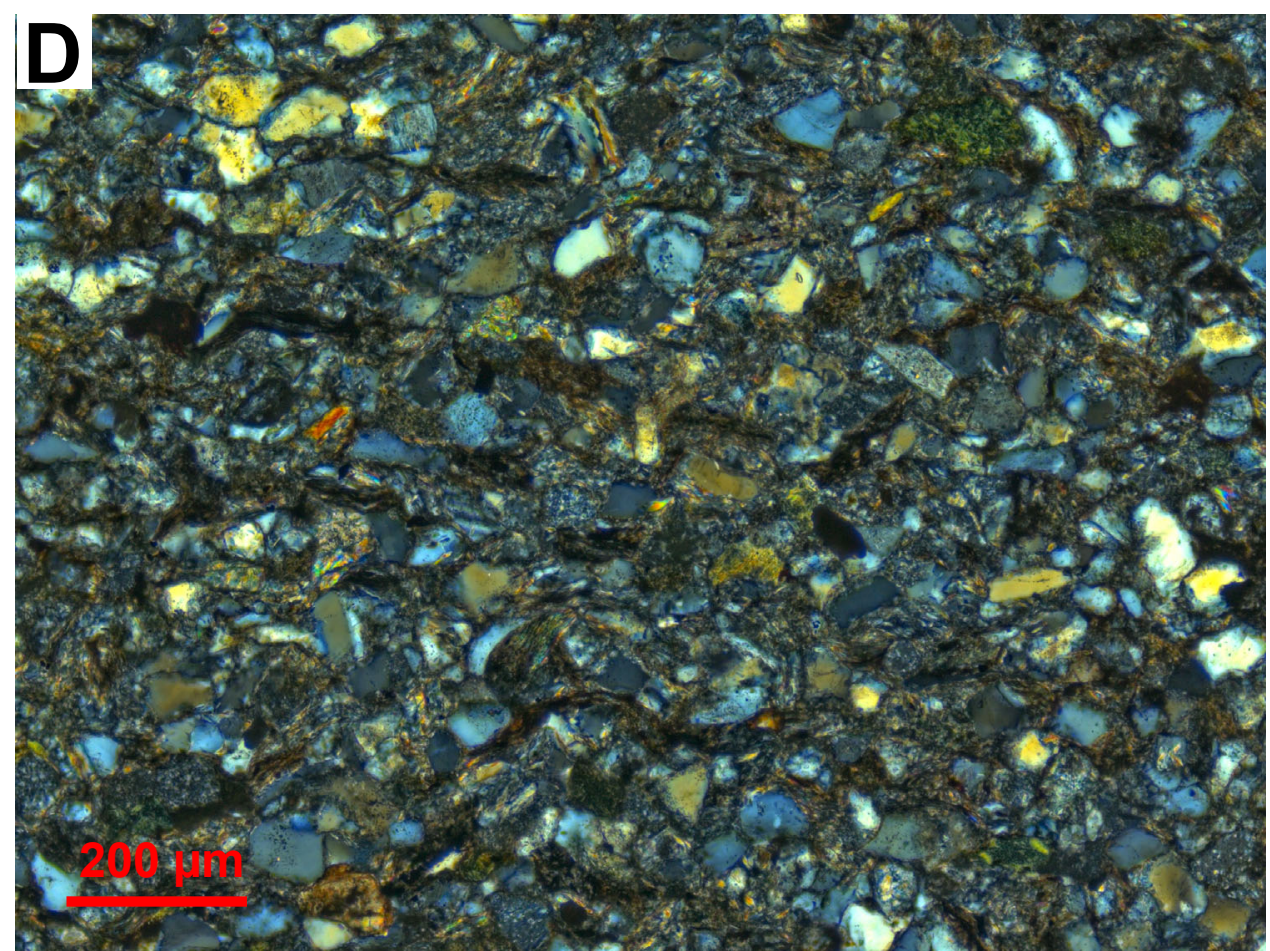



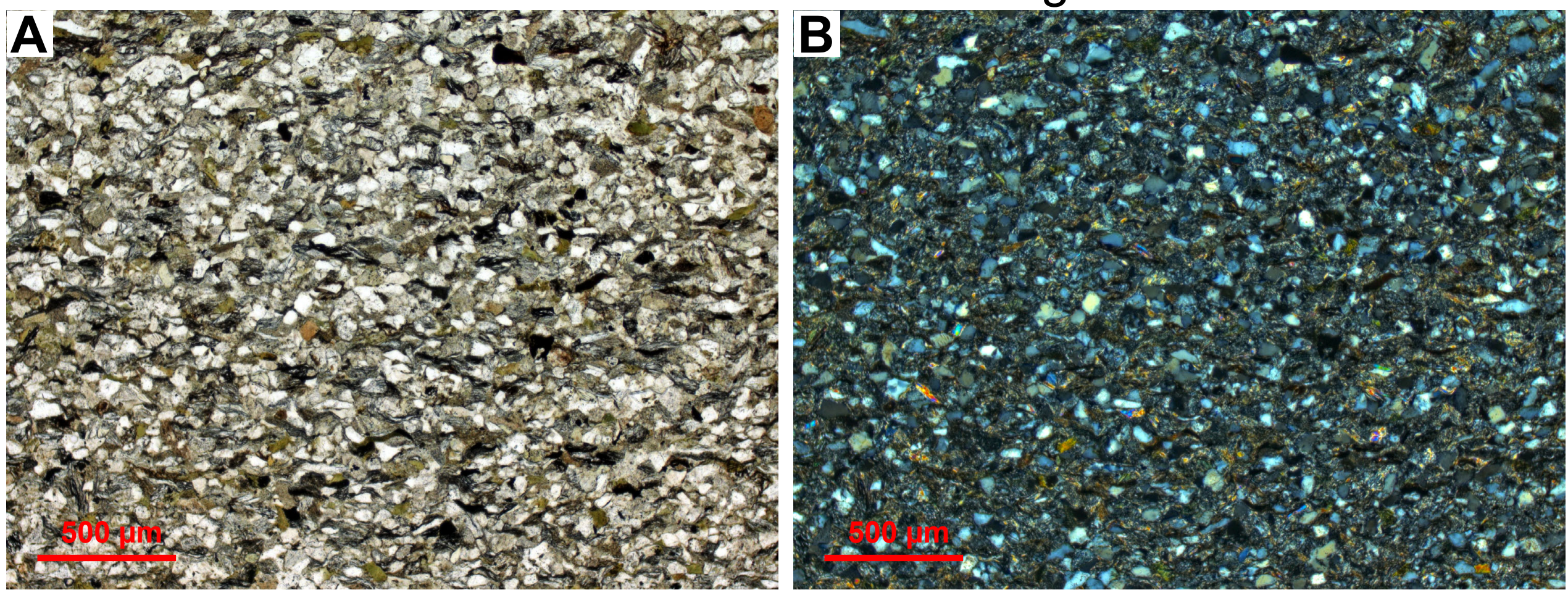

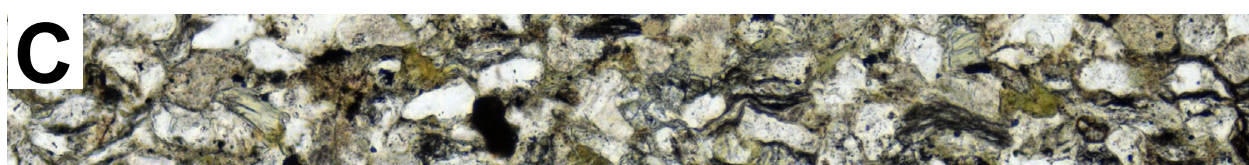

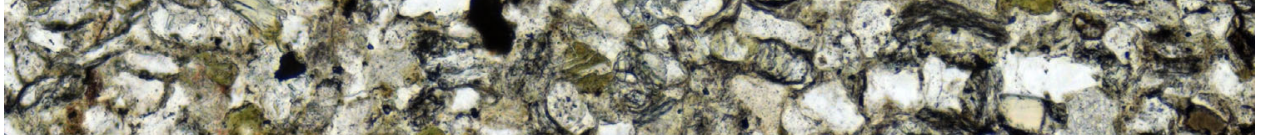

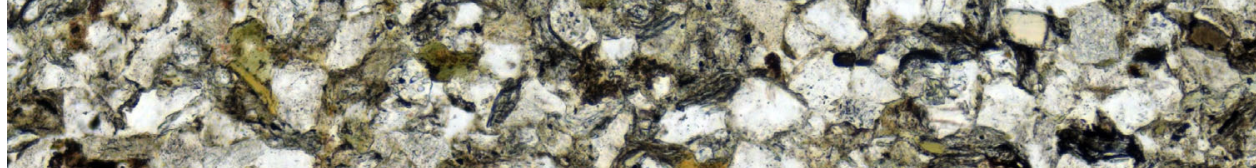
$2.5,265.5$ 5.2.

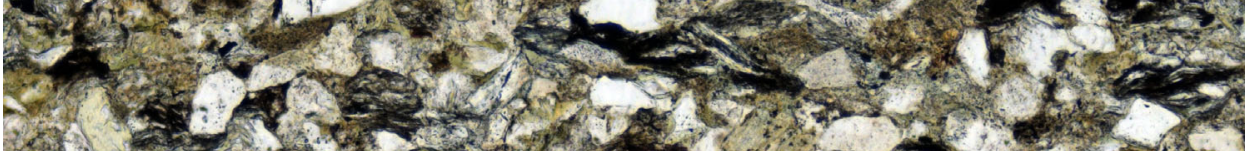

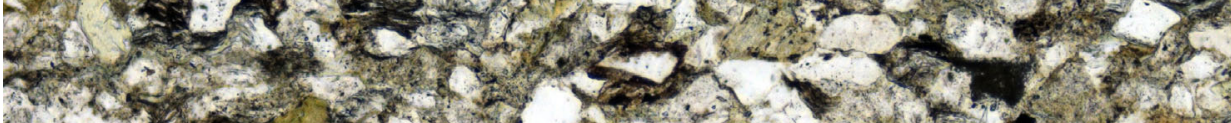

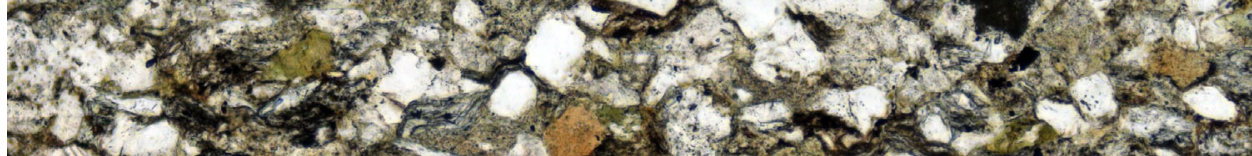

502015

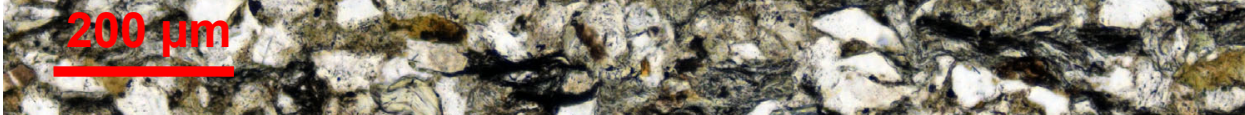

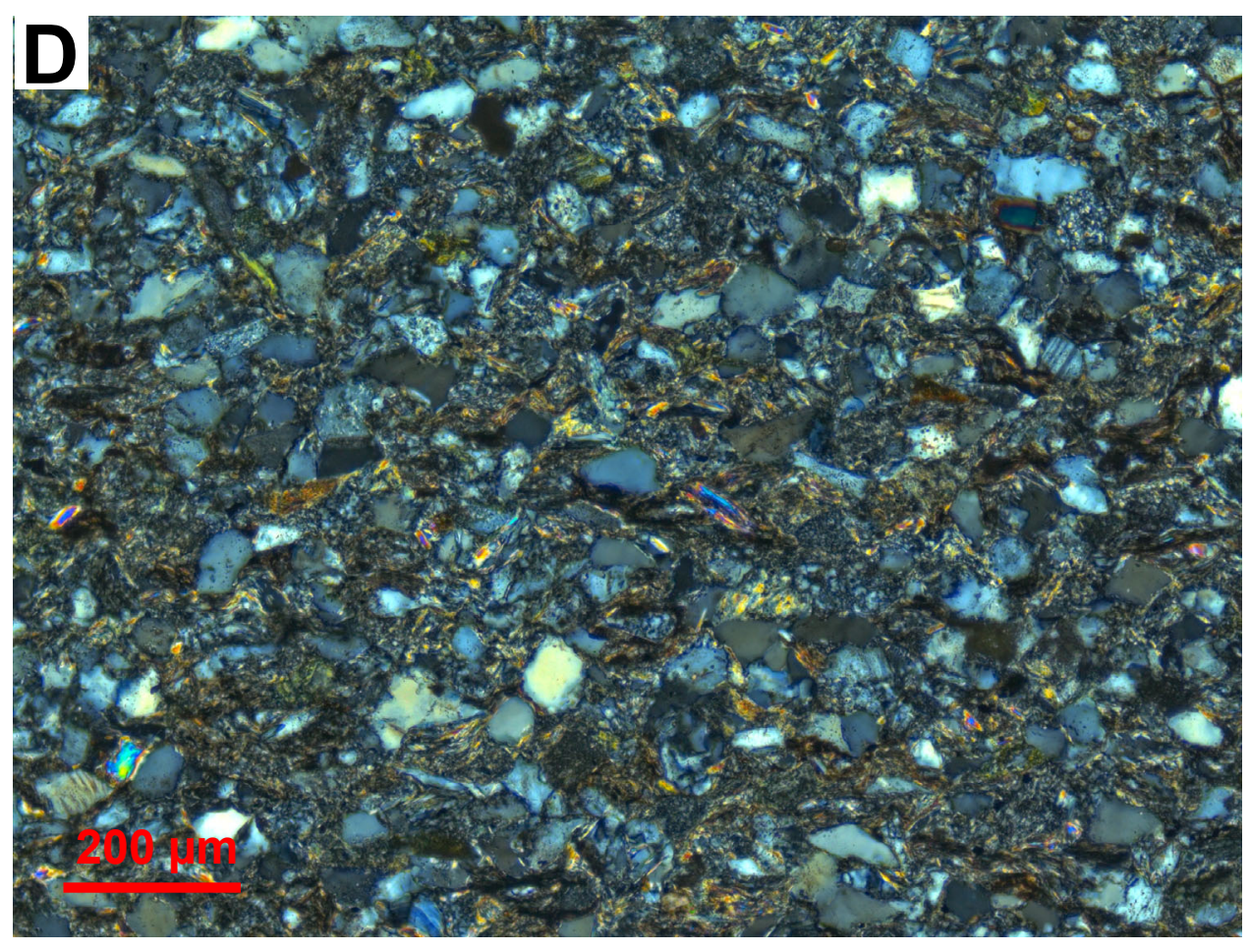


Fig 030 - 00DL024-064.0

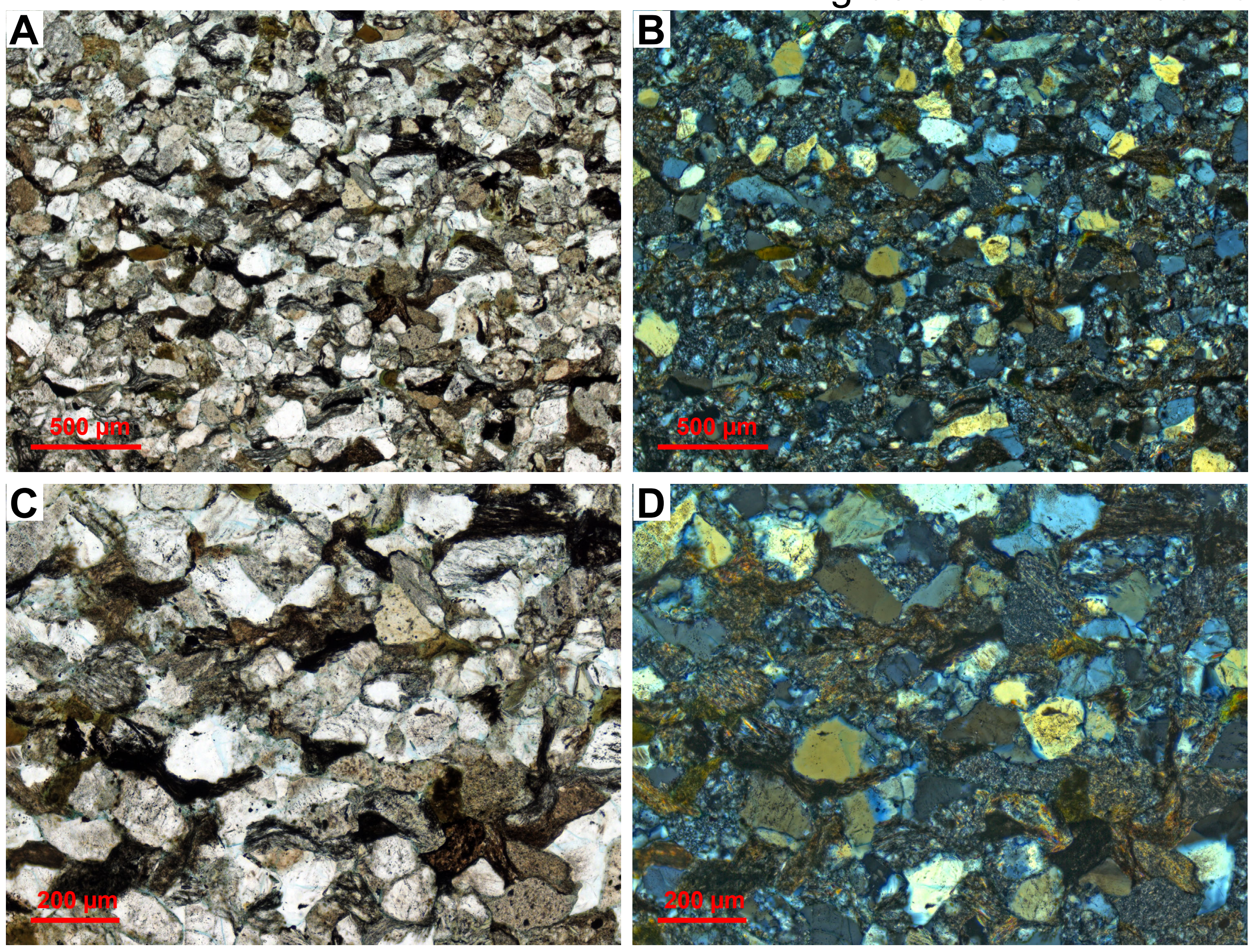


Fig 031 - 00DL024-072.8
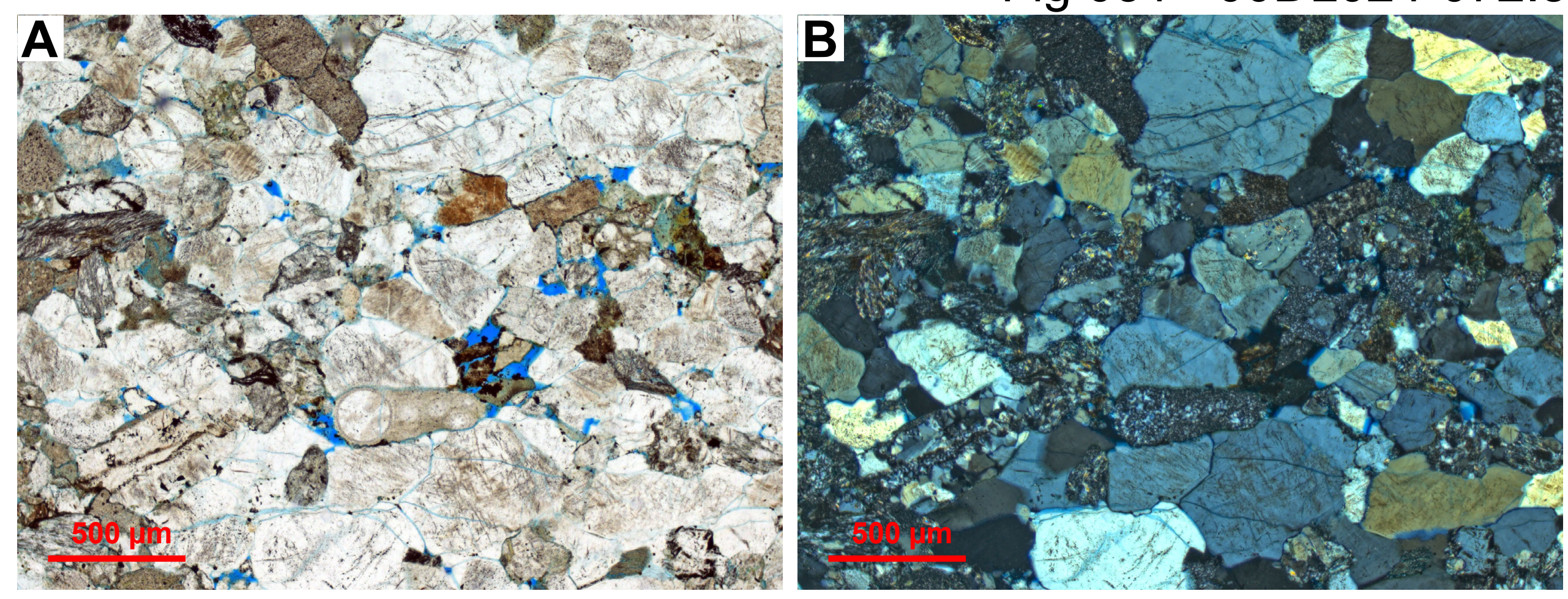

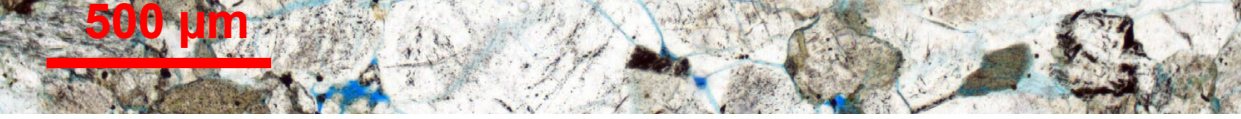
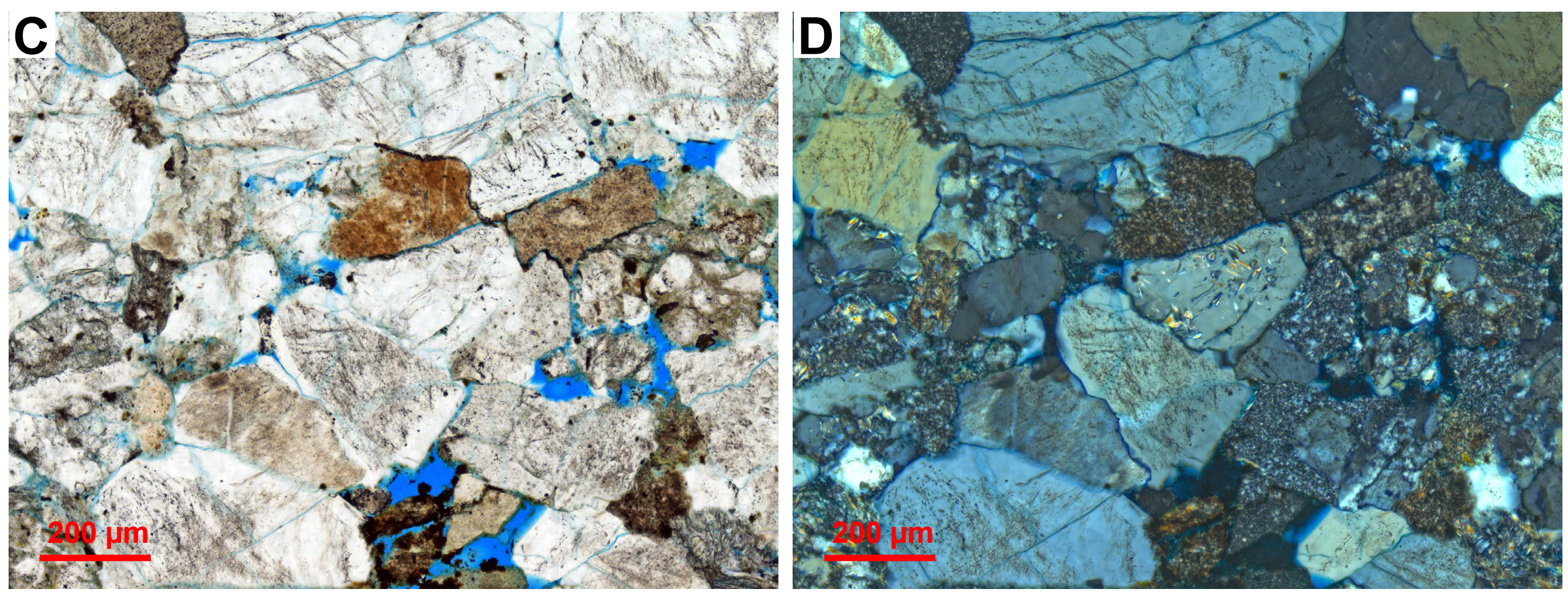
Fig 032 - 00DL024-082.3
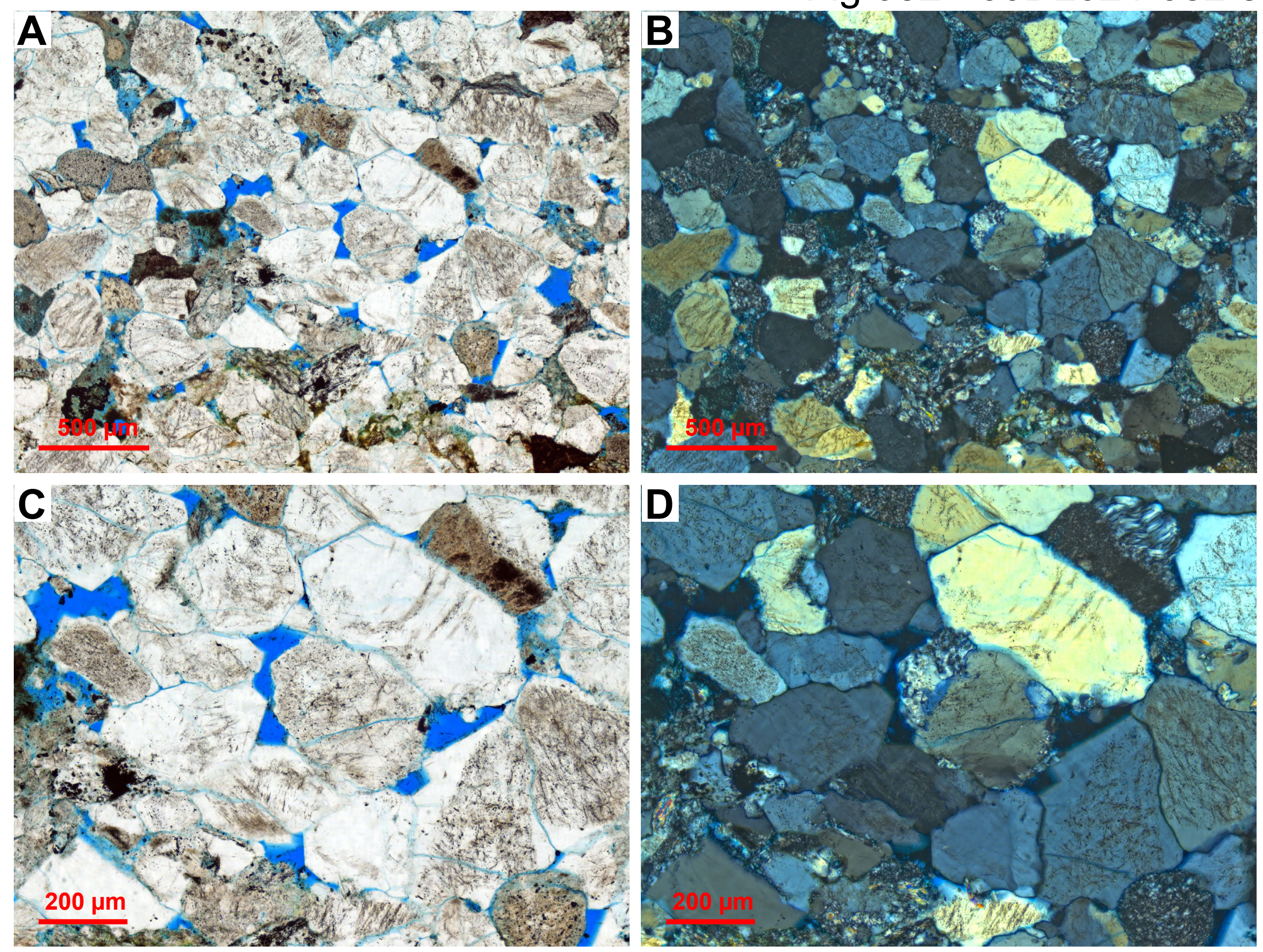
Fig 033 - 19DL019-004.5A
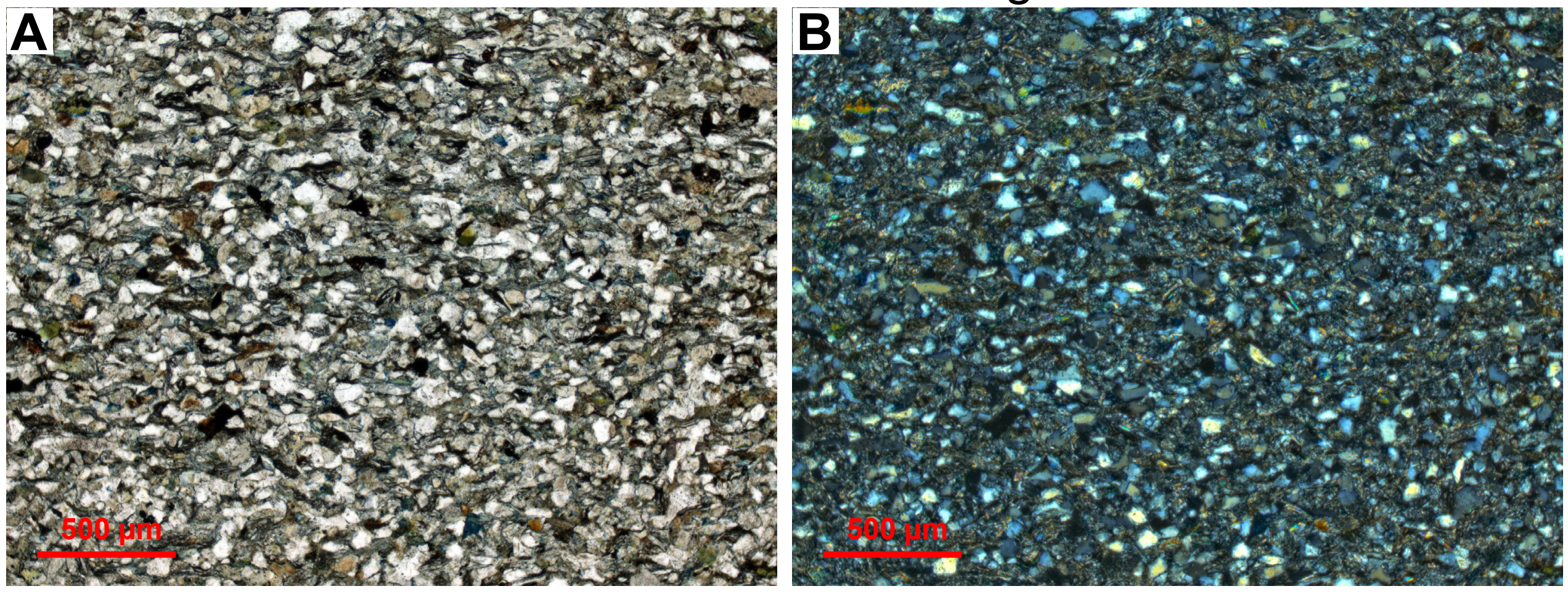

C 5

2.7.

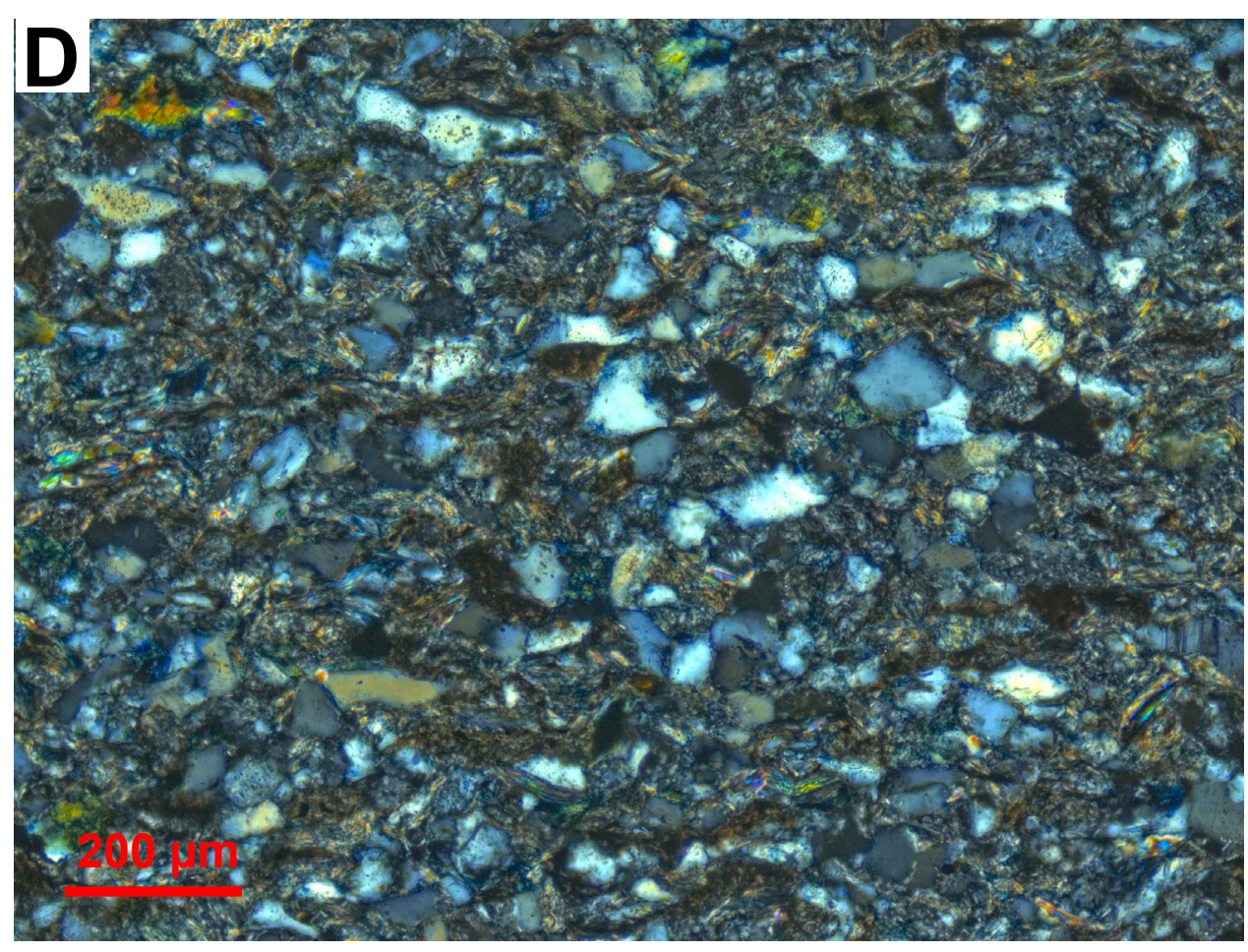


Fig 034 - 19DL019-008.0A

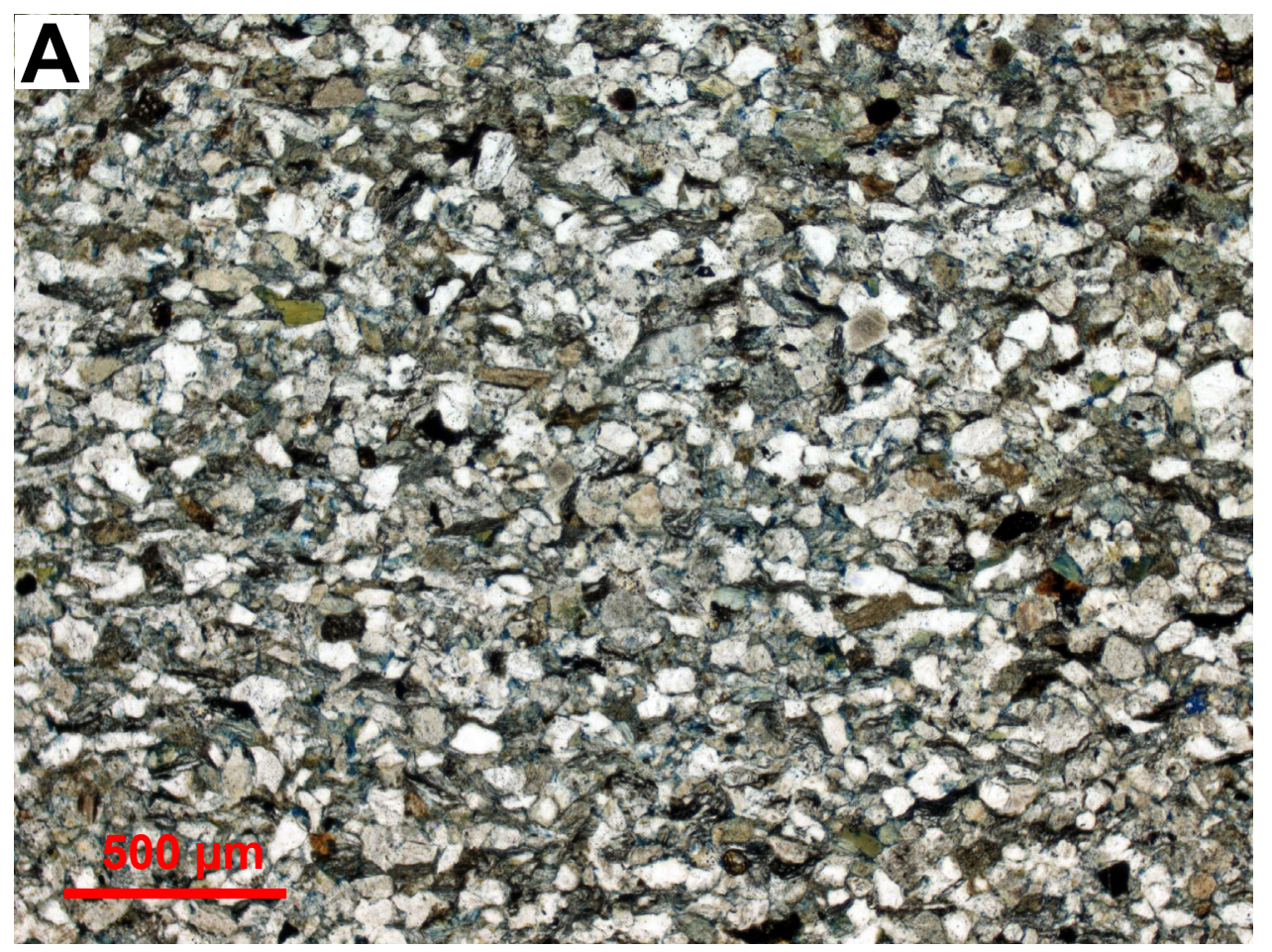

(2)

CP.

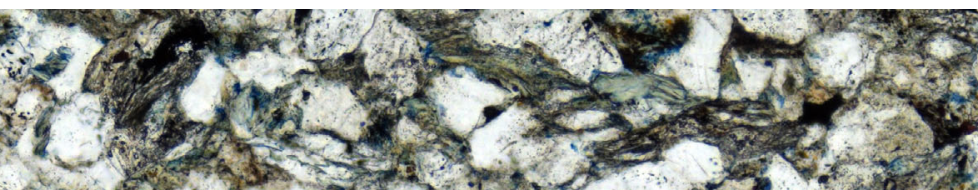

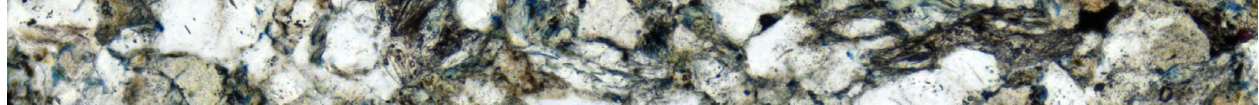

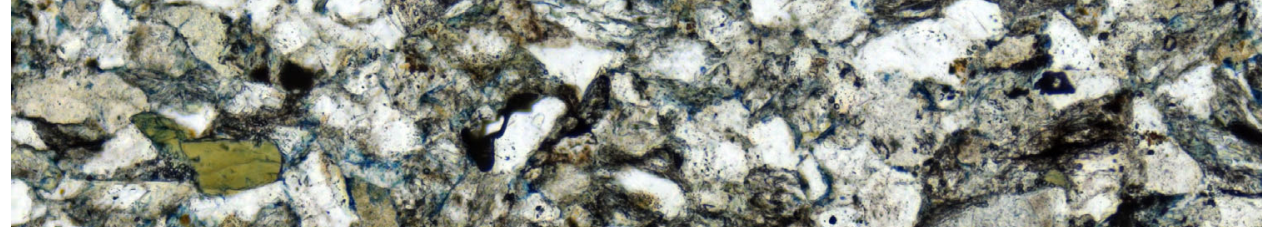

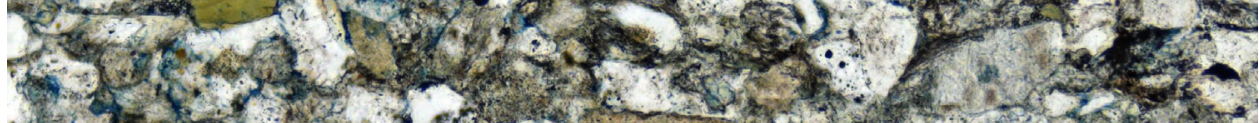

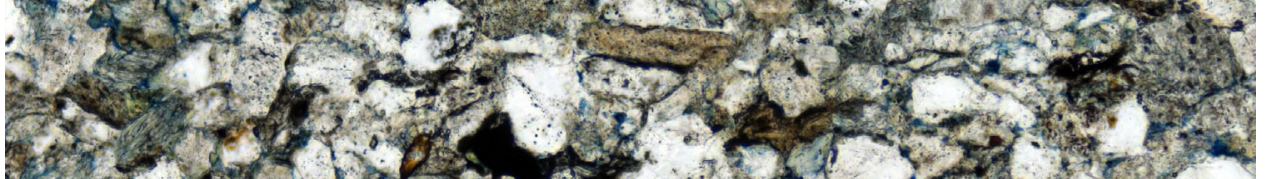

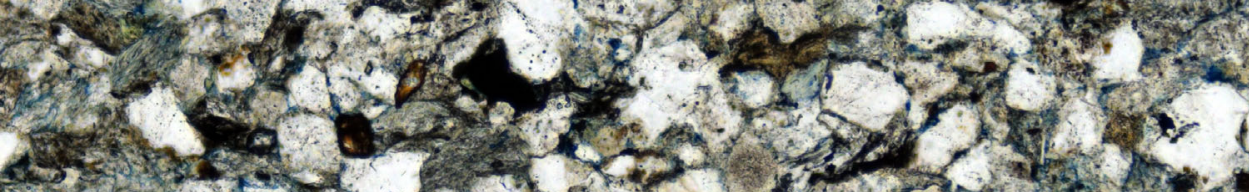

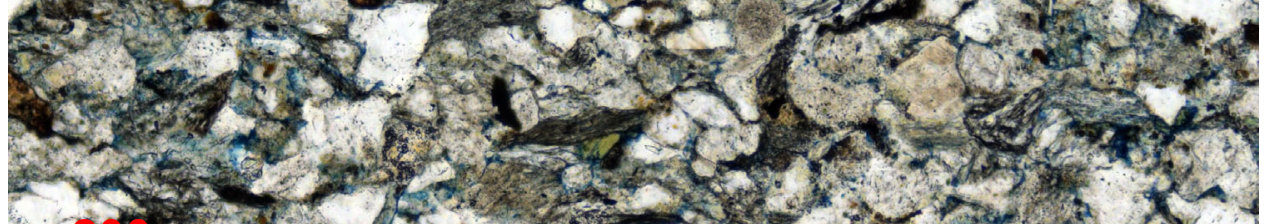

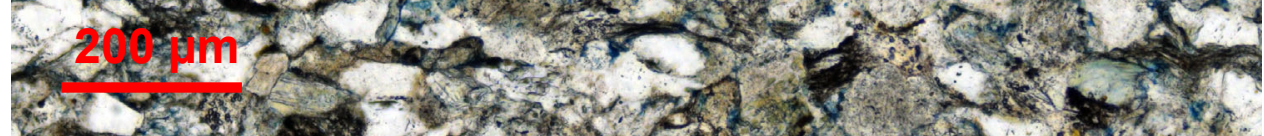

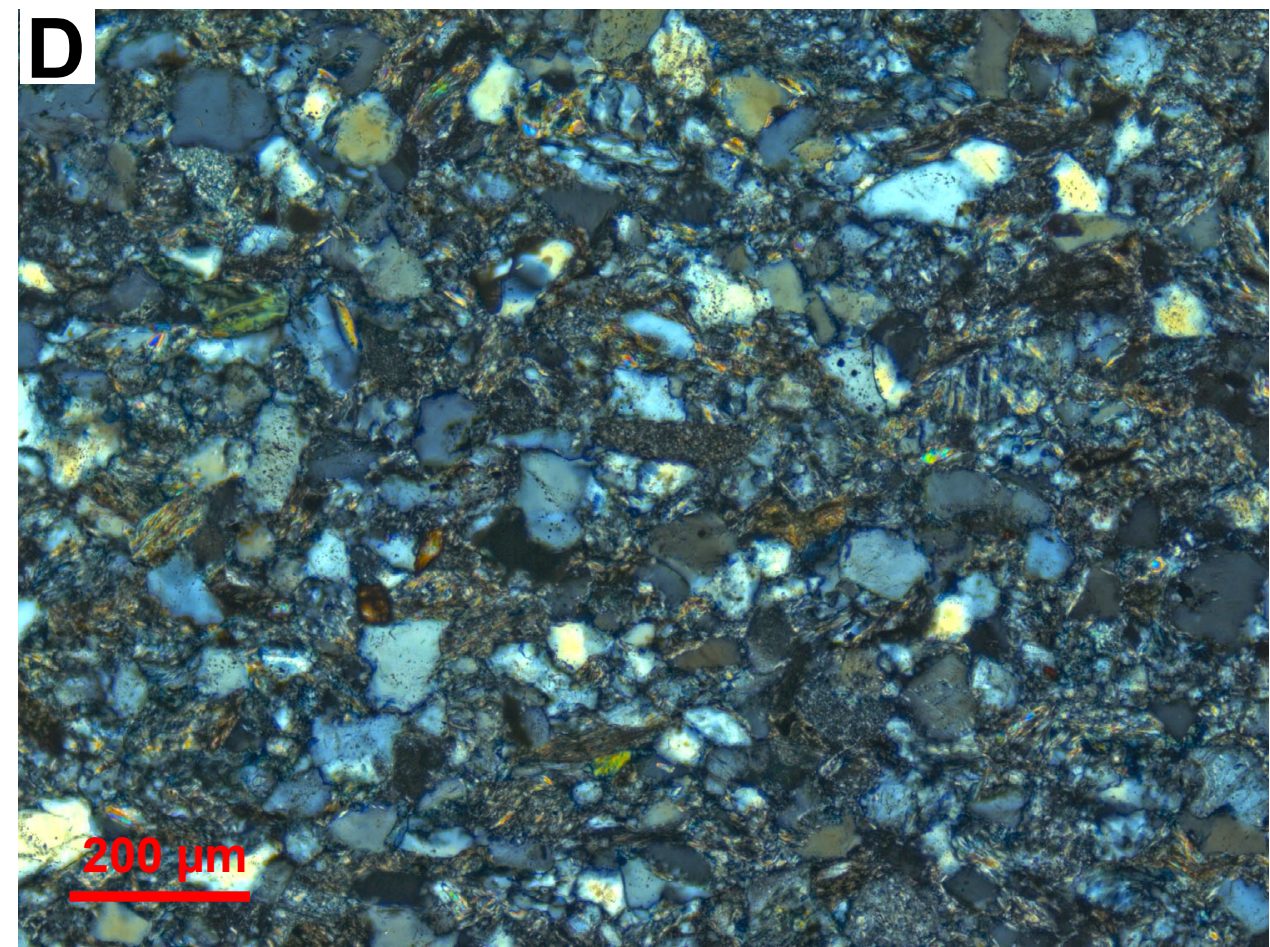


Fig 035 - 19DL019-190.0A

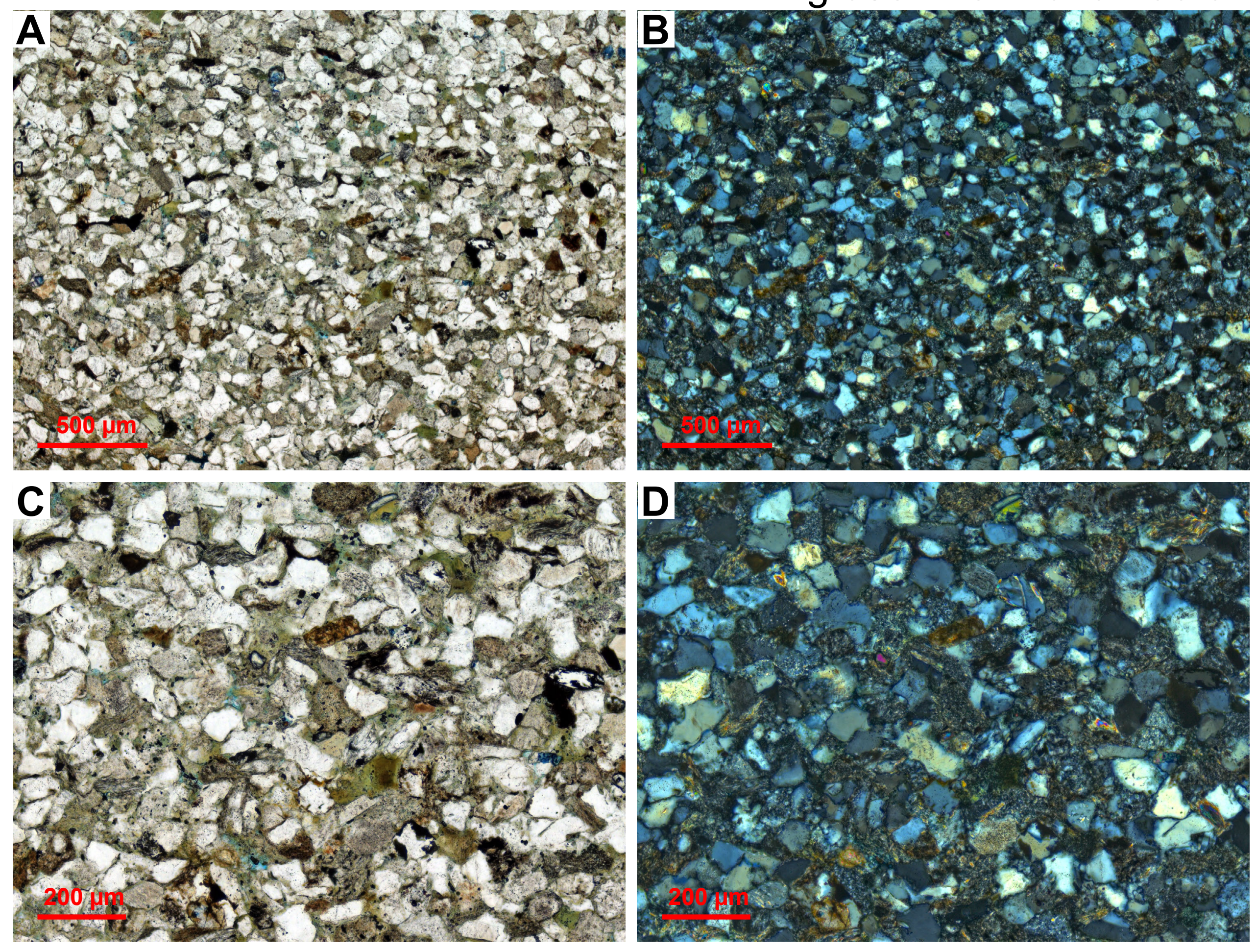


Fig 036 - 19DL019-235.8A

A 15
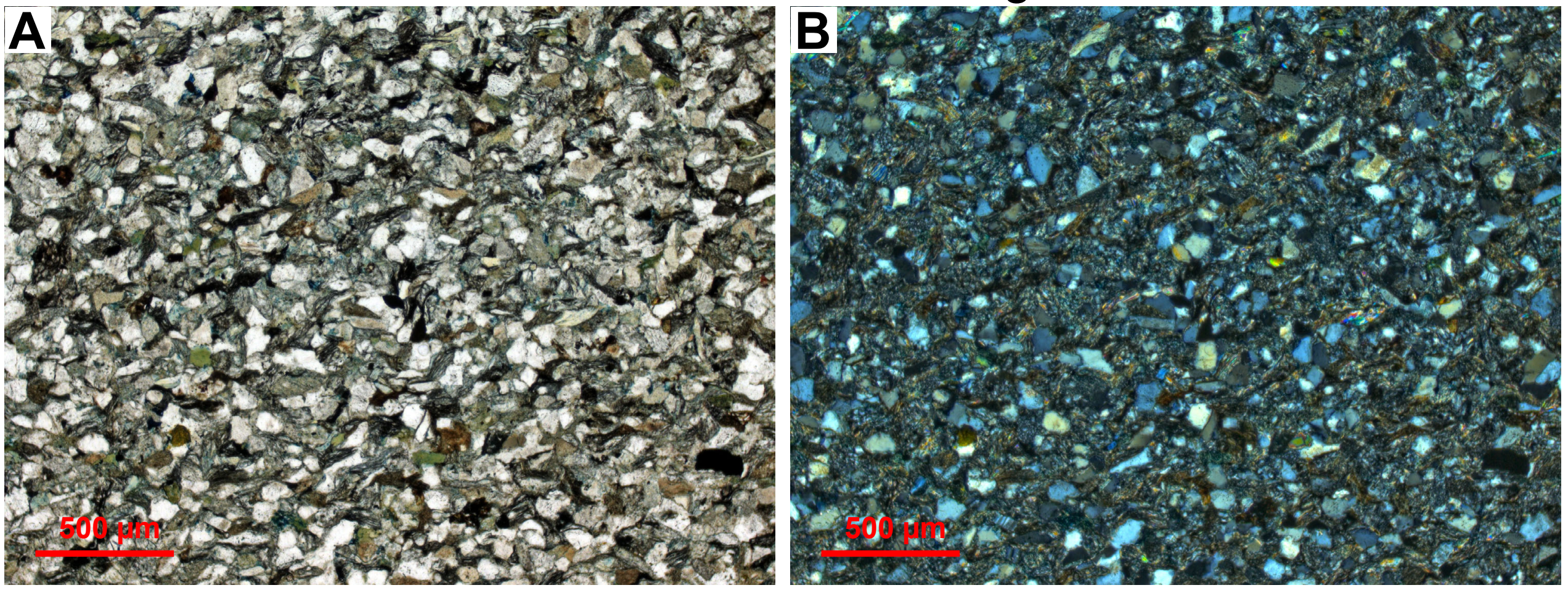

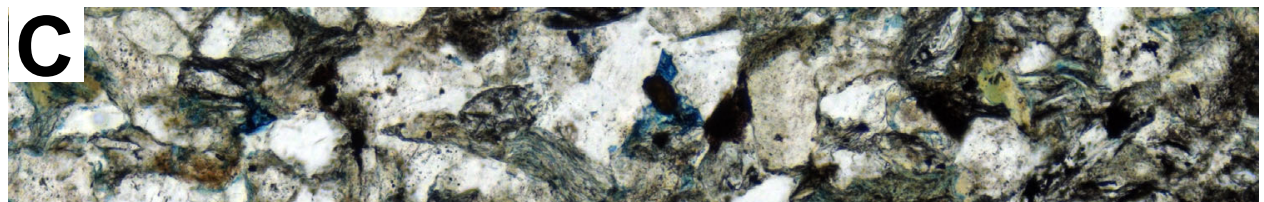

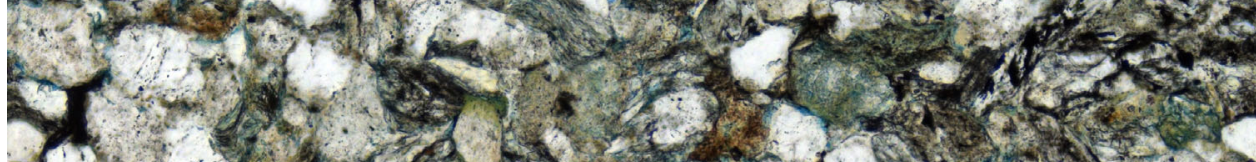

ros 501

2.127,

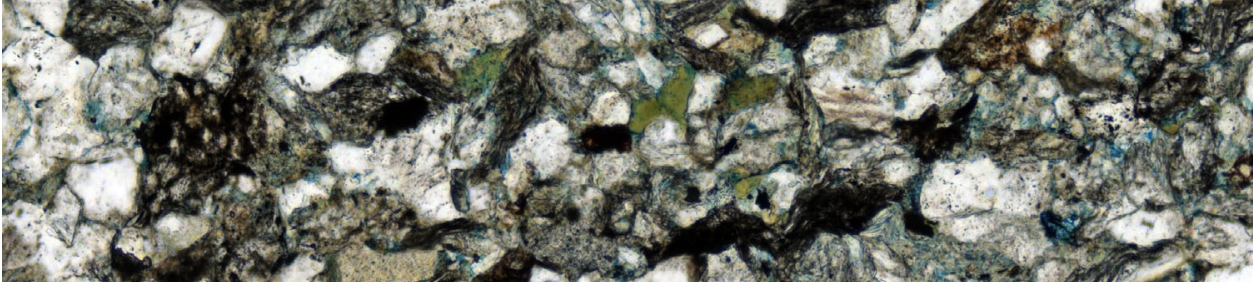

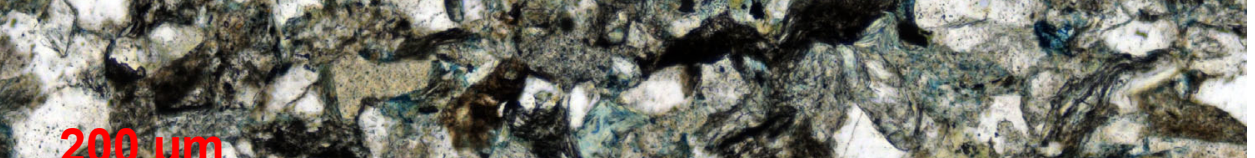
2.

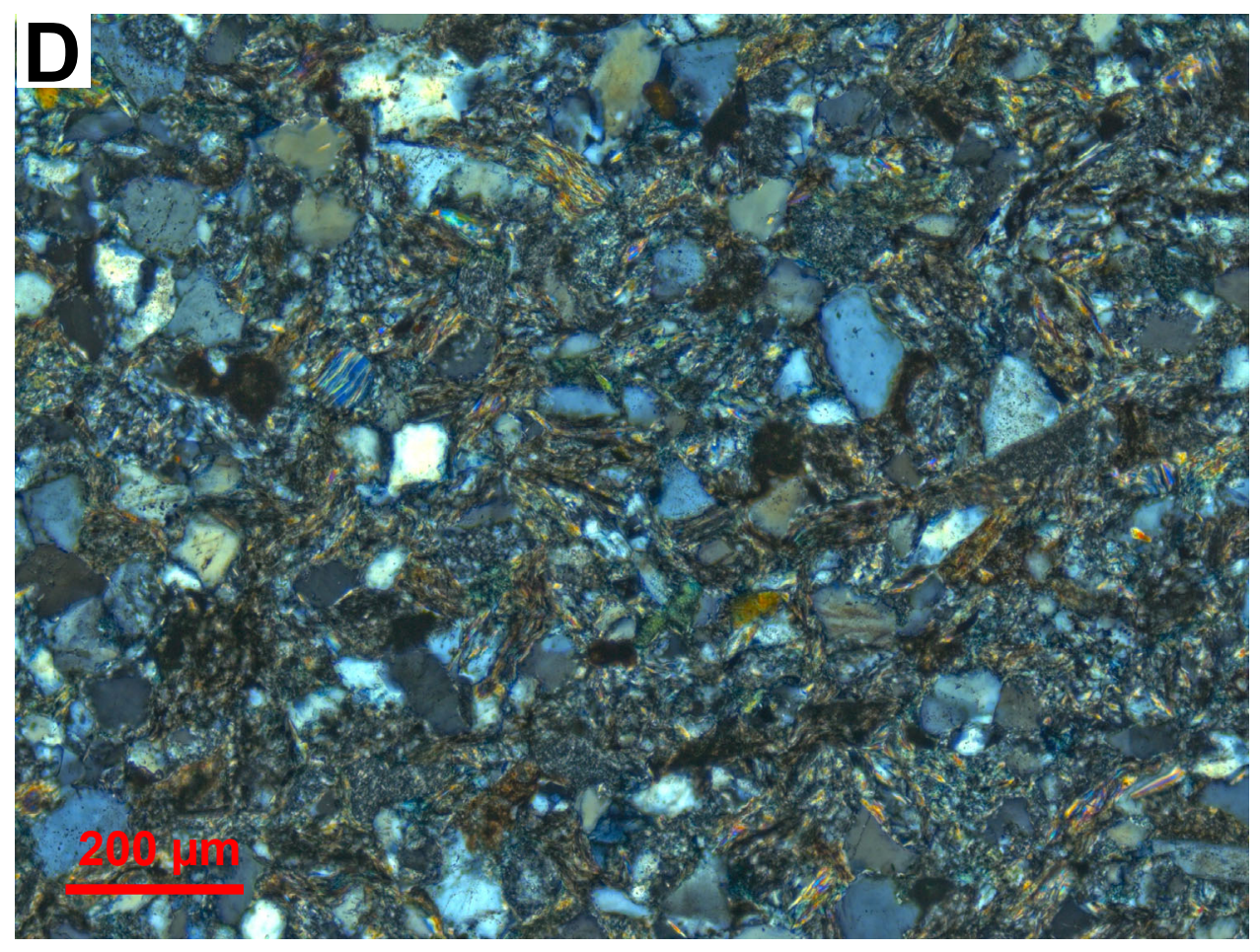


Fig 037 - 19DL020-001.0A

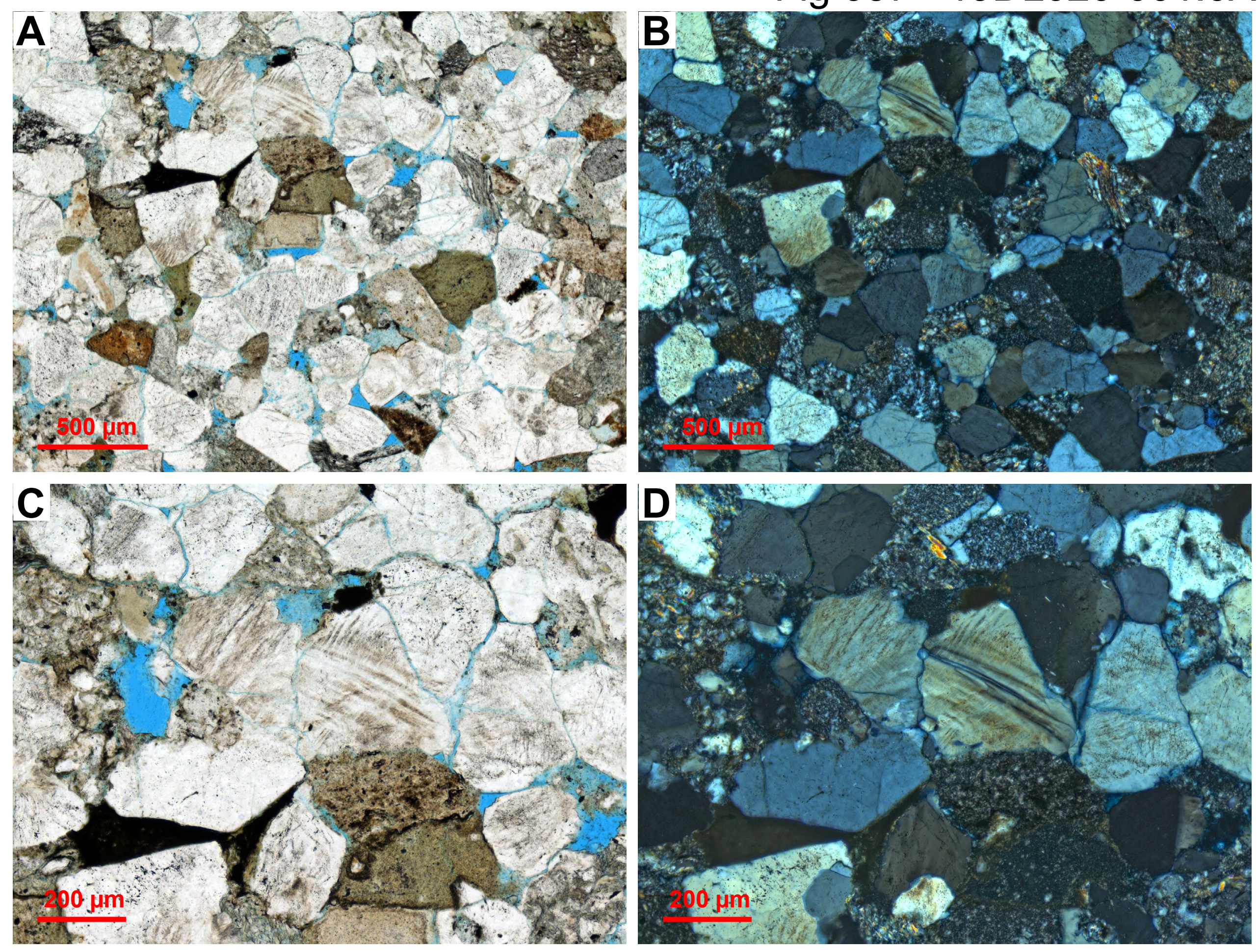


Fig 038 - 99DL064-021.5
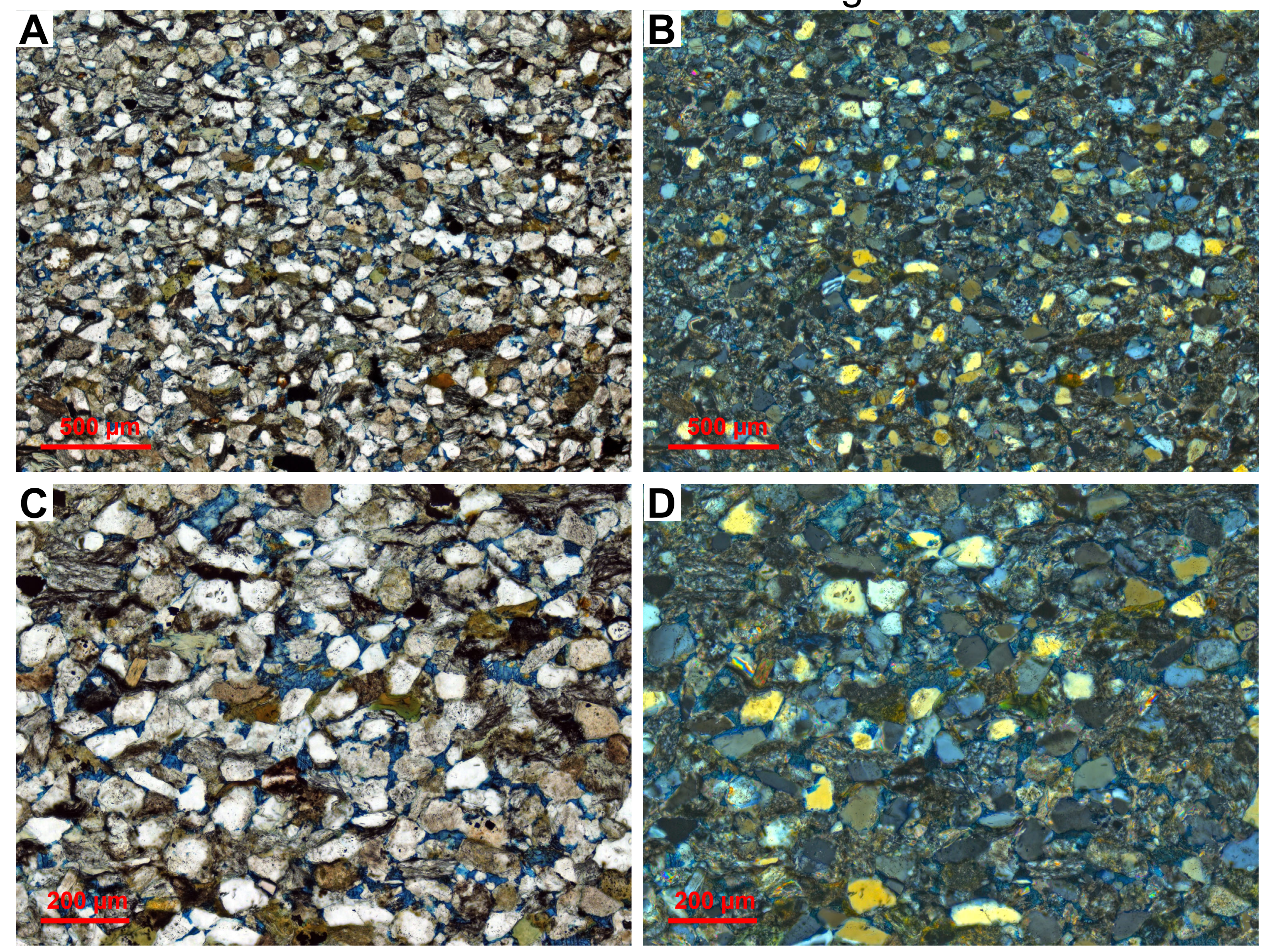
Fig 039 - 99DL064-022.4
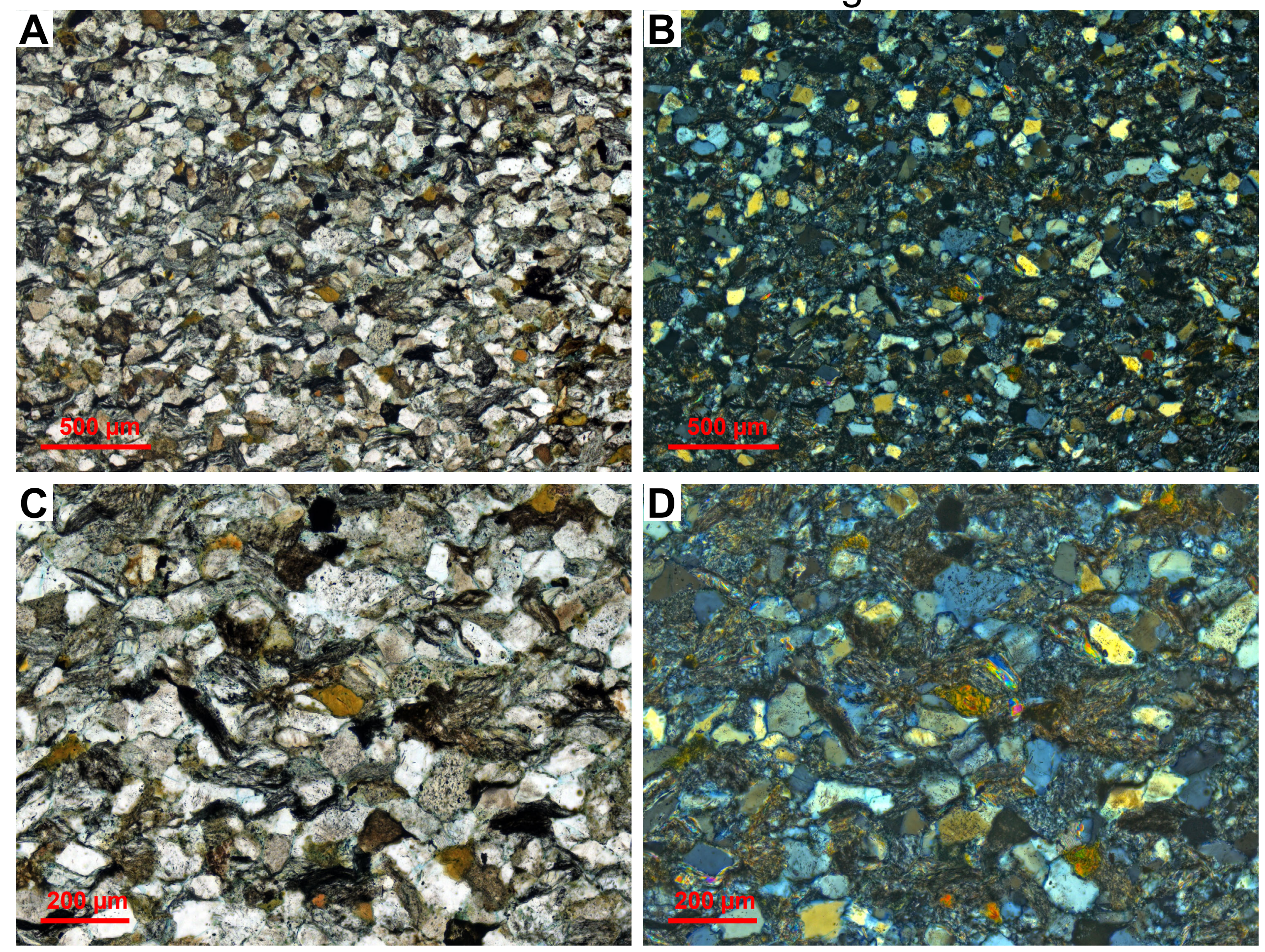
Fig 040 - 99DL064-064.5
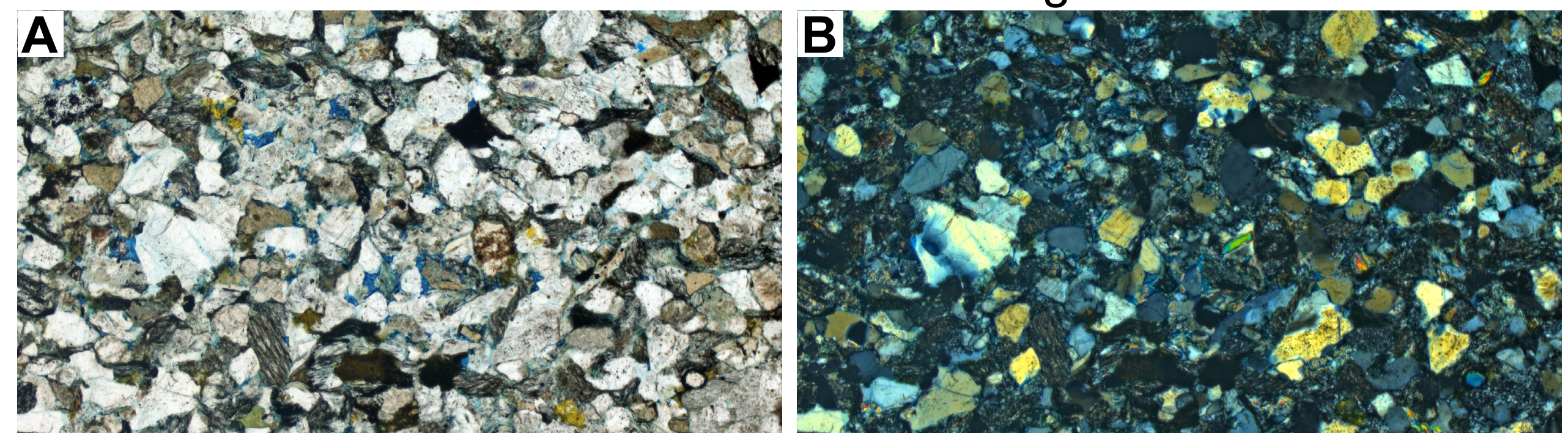

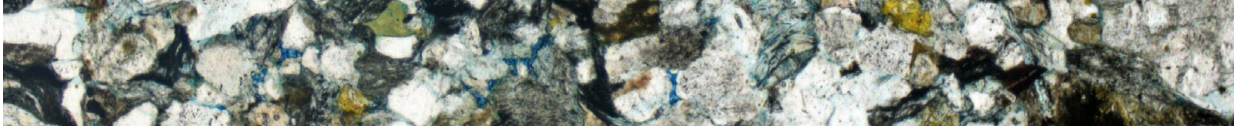
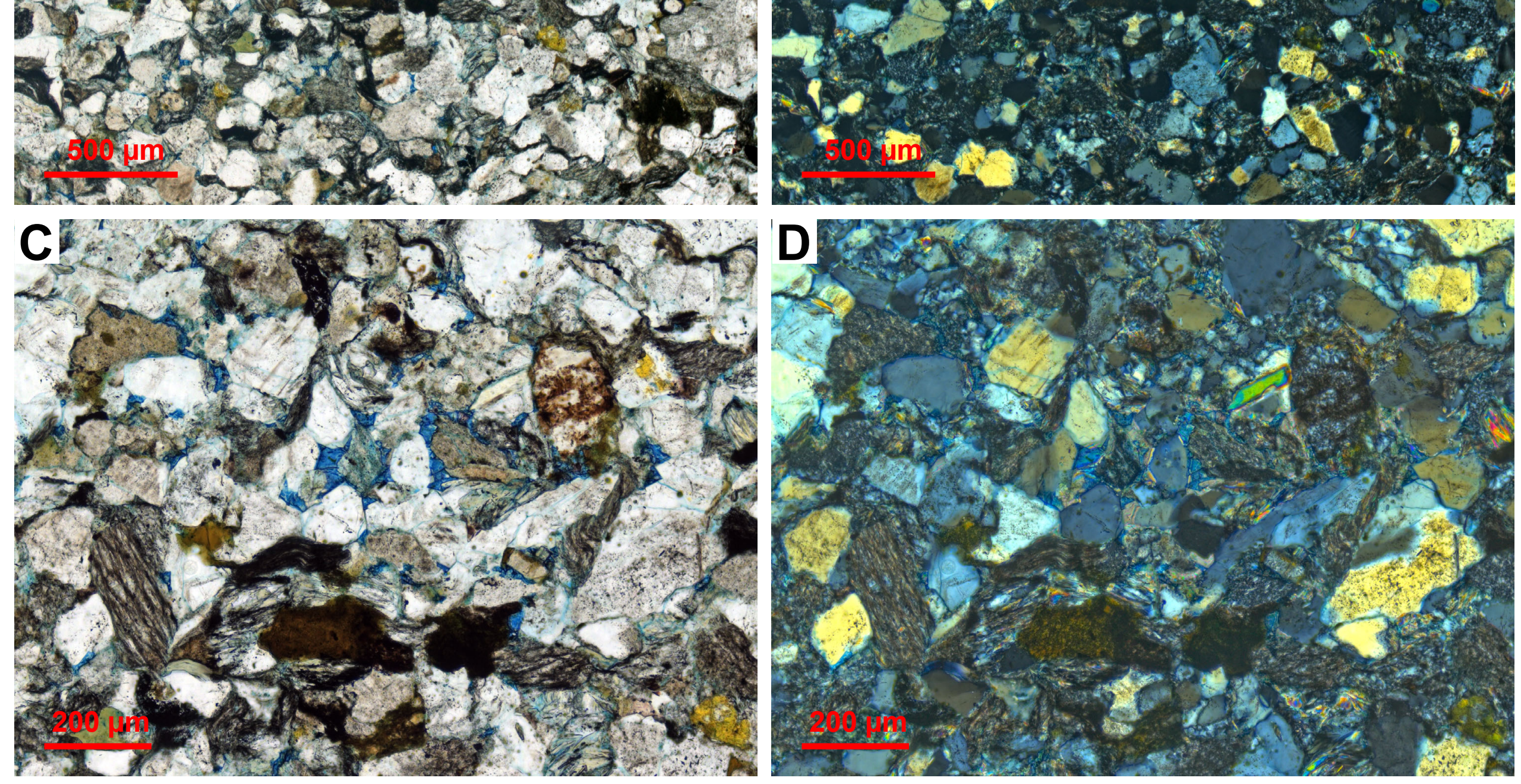
Fig 041 - 99DL064-172.2

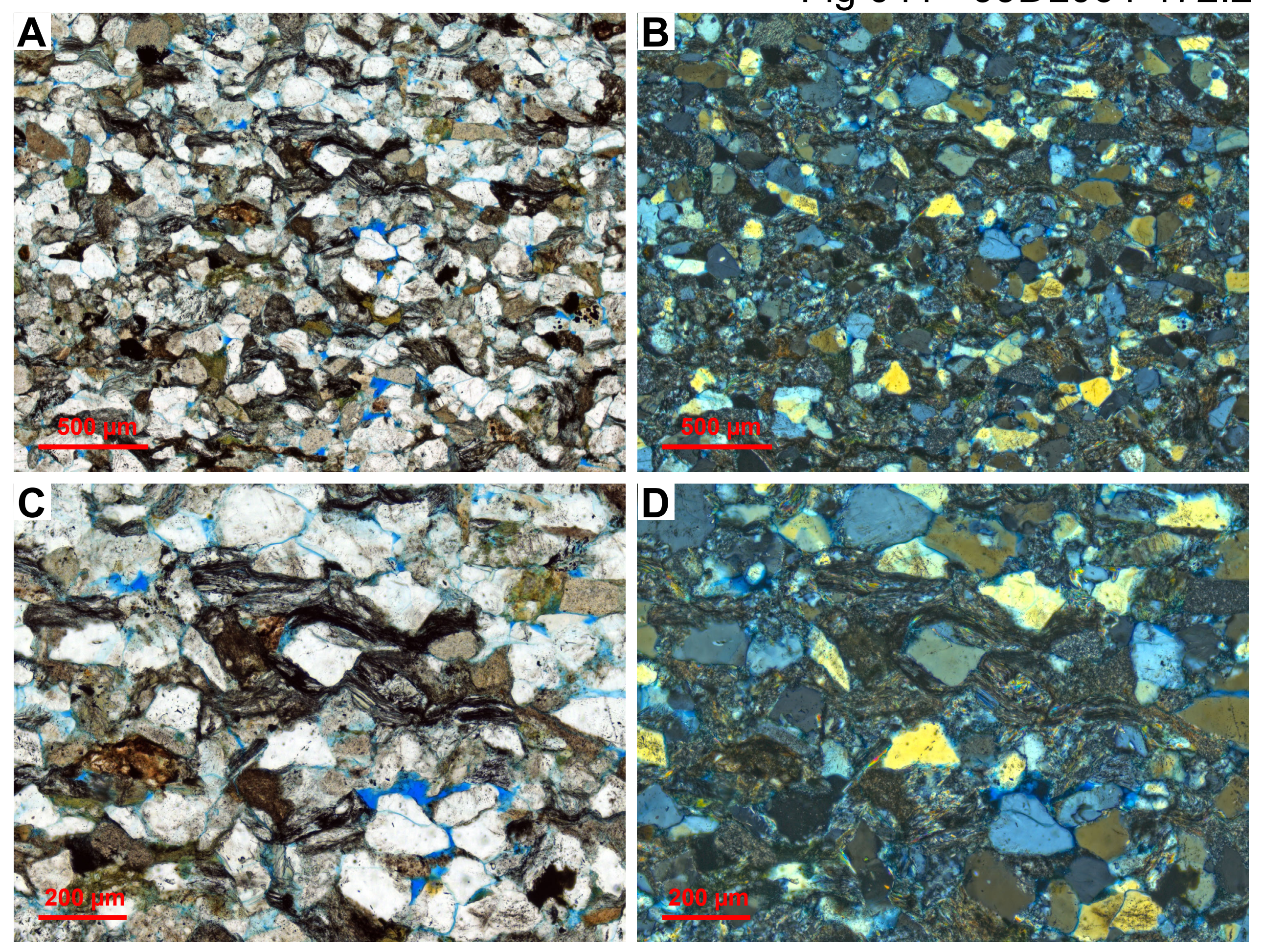


Fig 042 - 99DL064-180.0
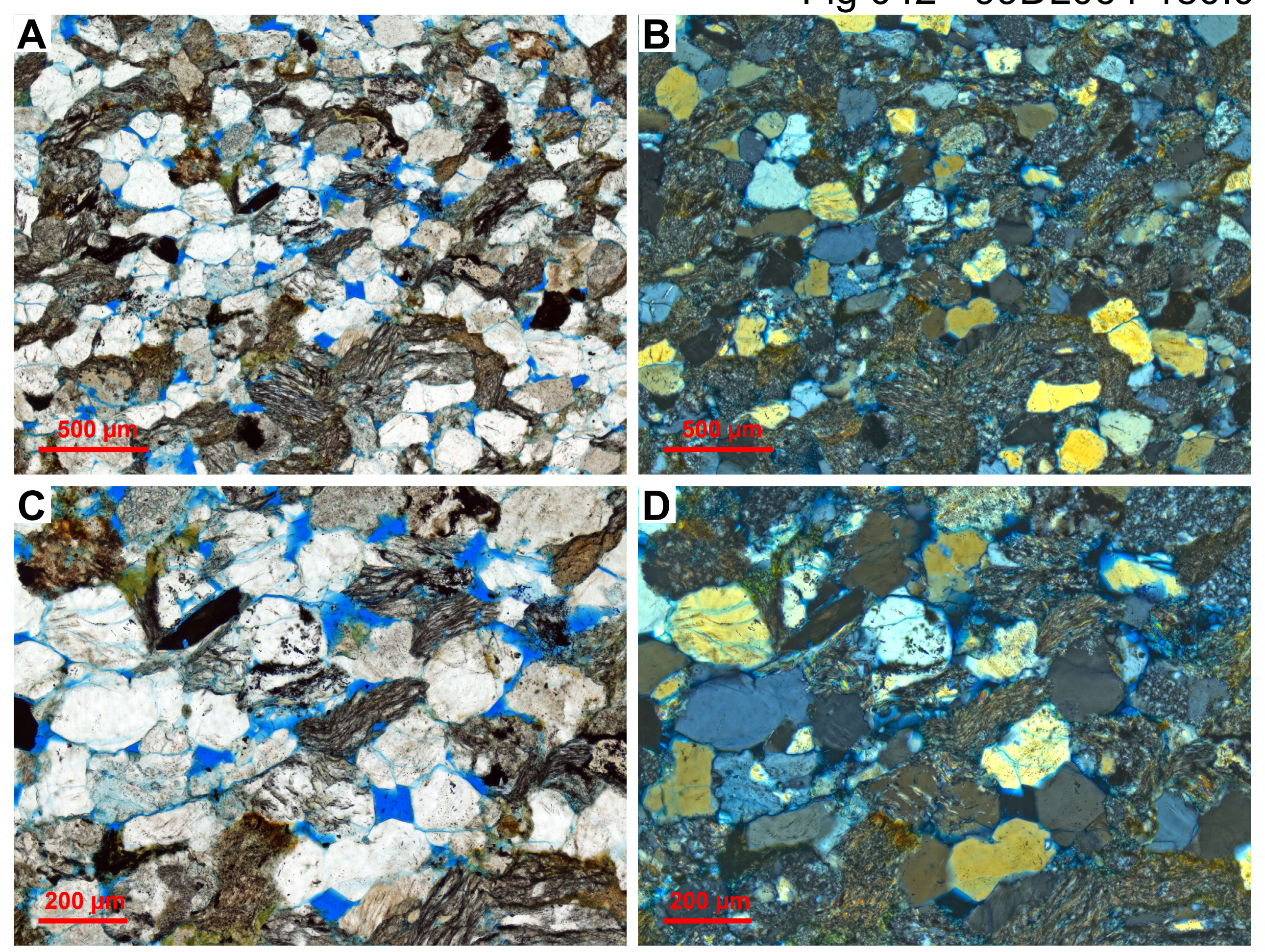
Fig 043 - 99DL064-230.4

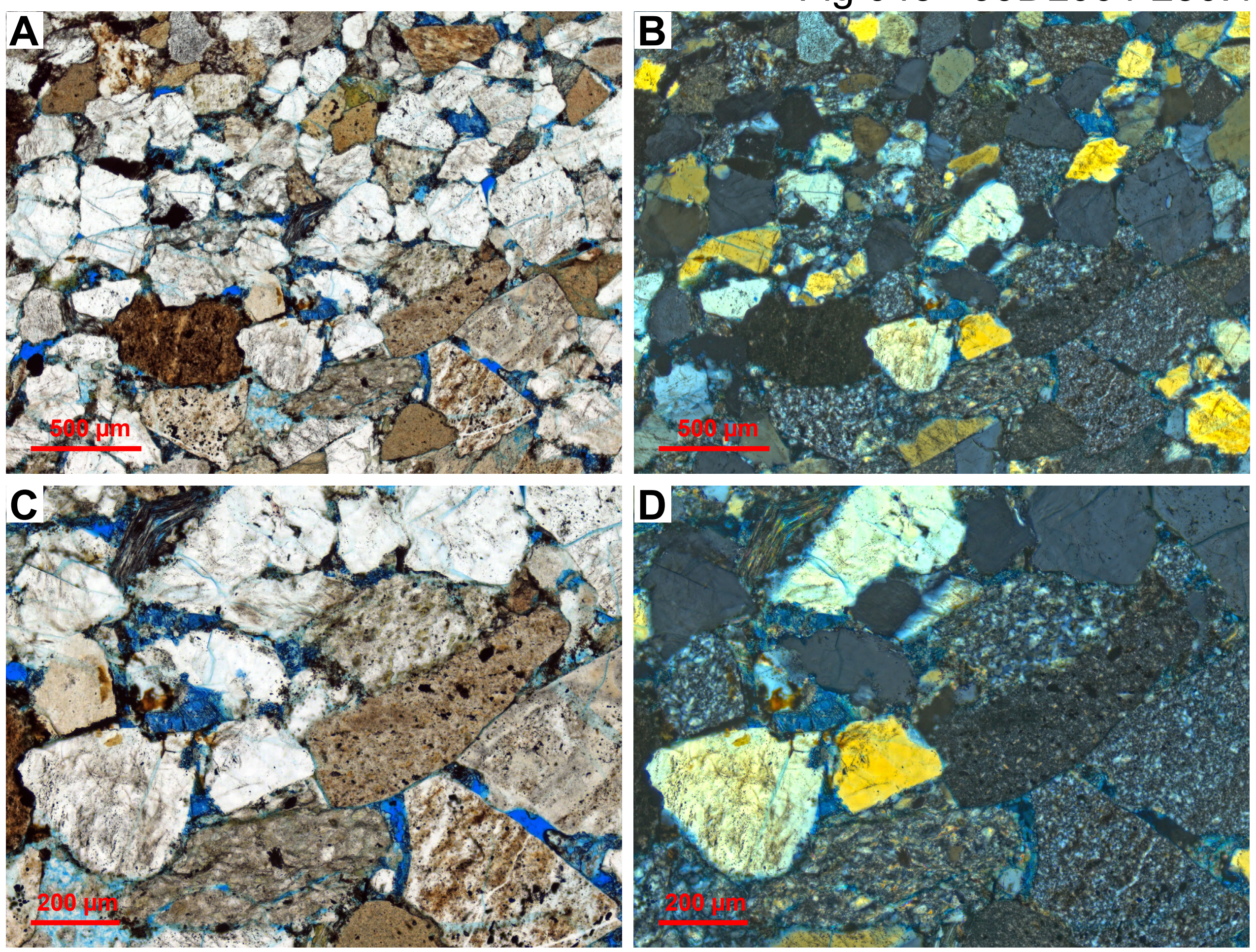


Fig 044 - 99DL064A-008.5

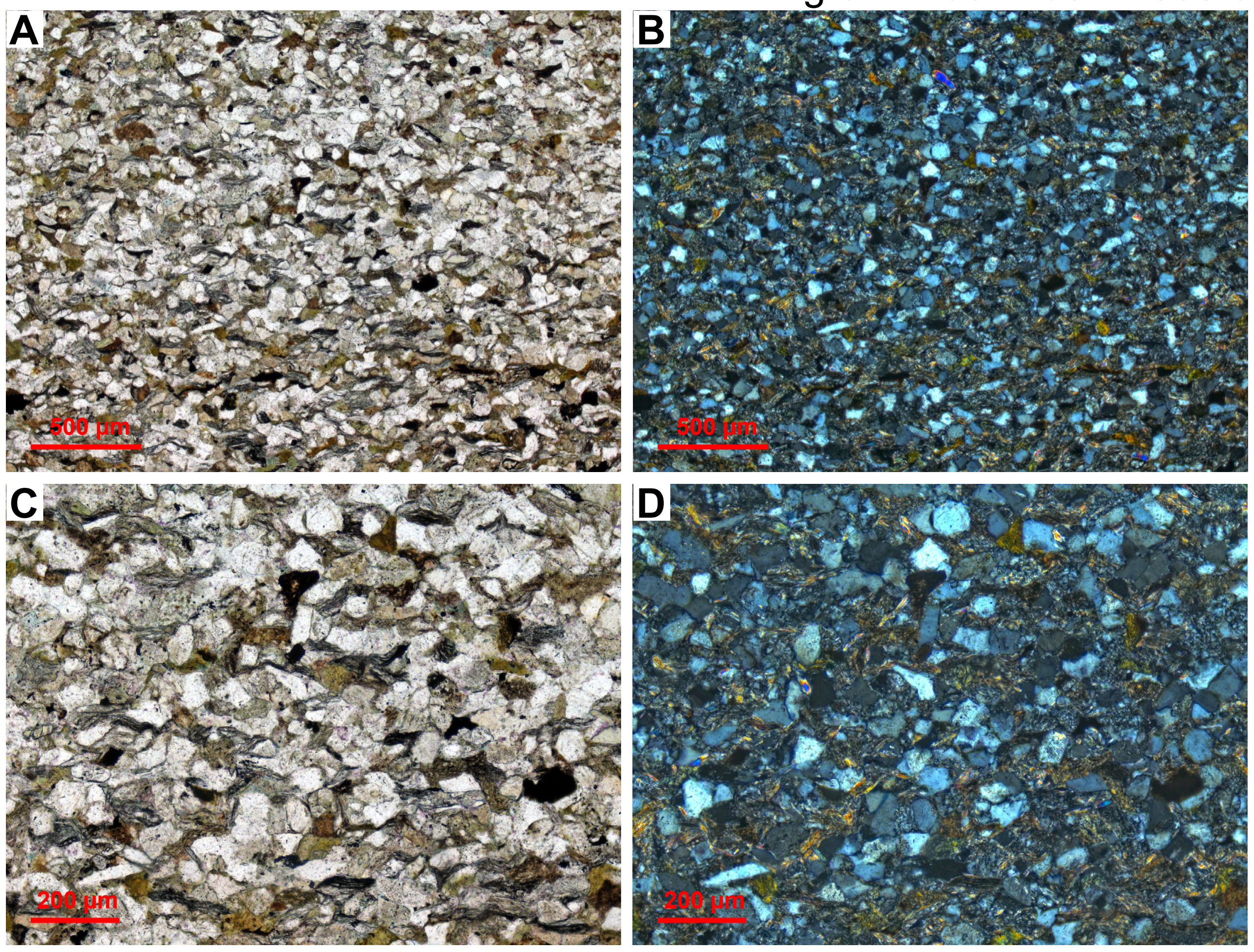




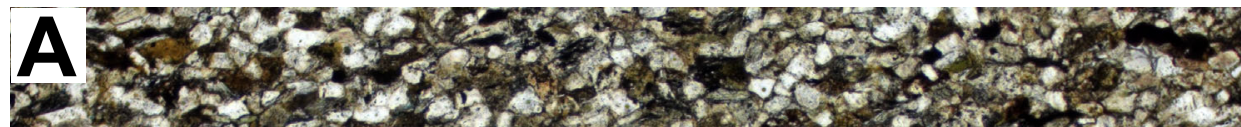

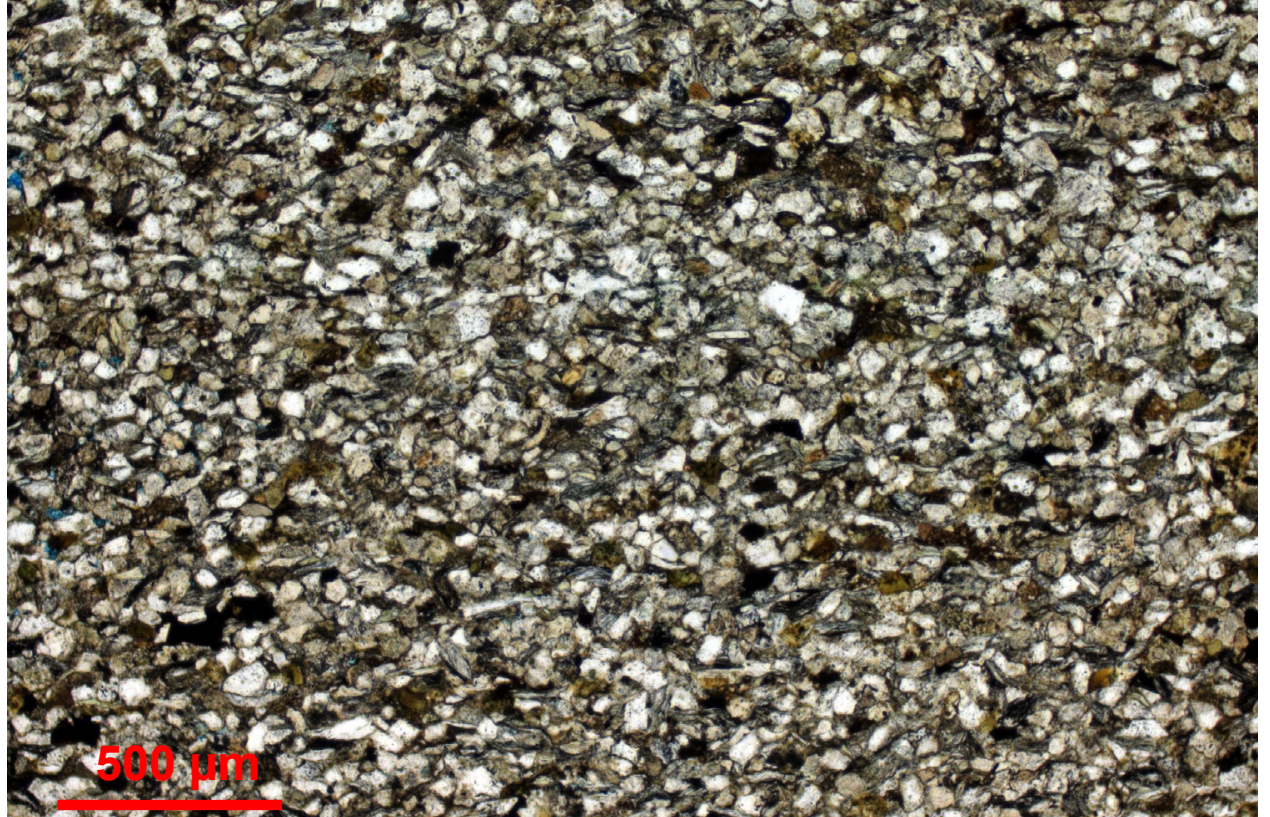

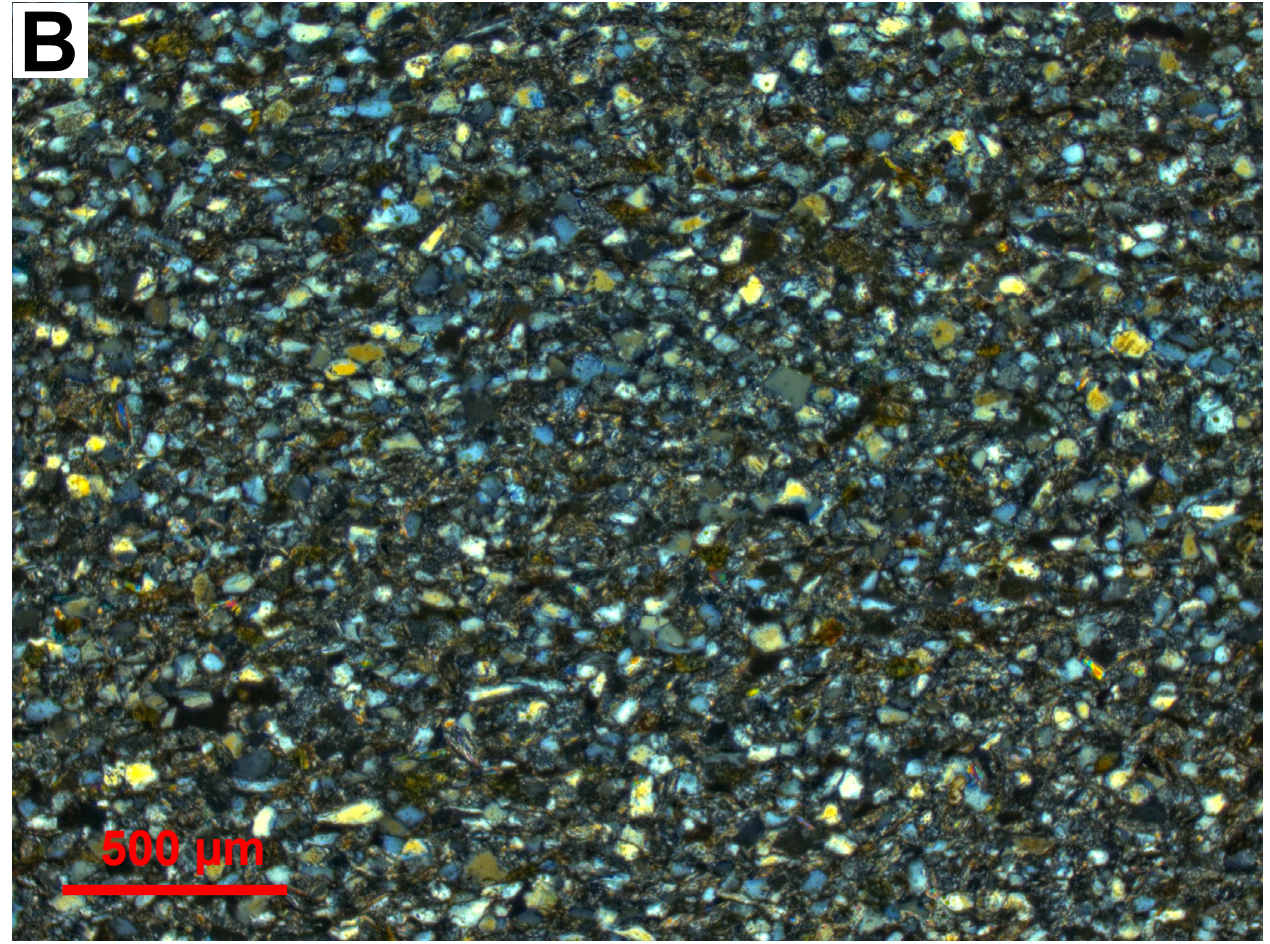

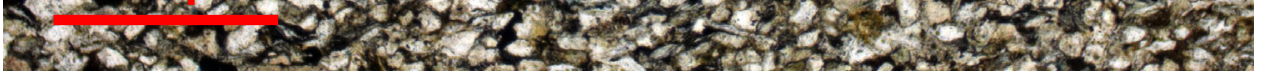

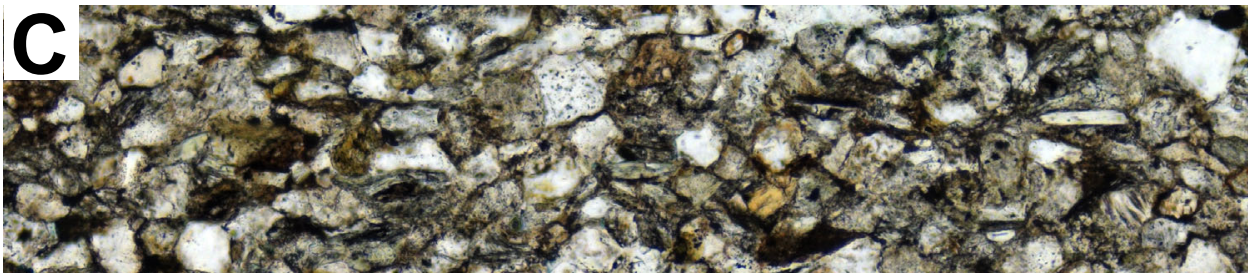

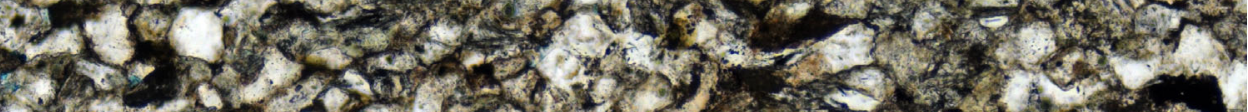

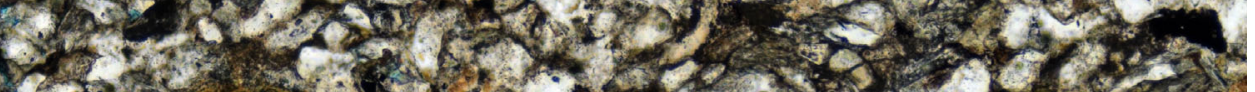

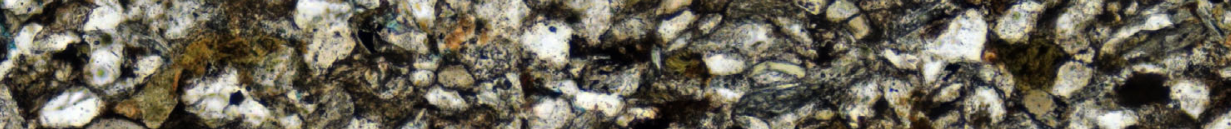

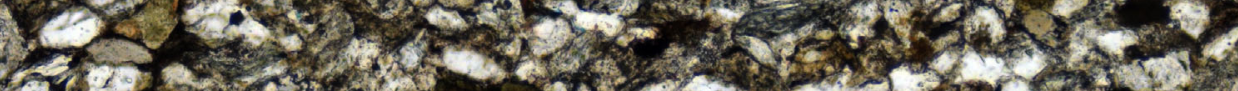

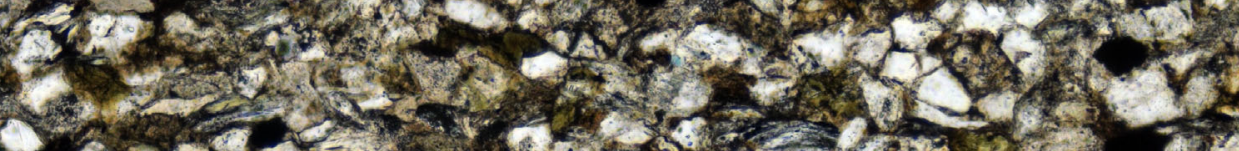

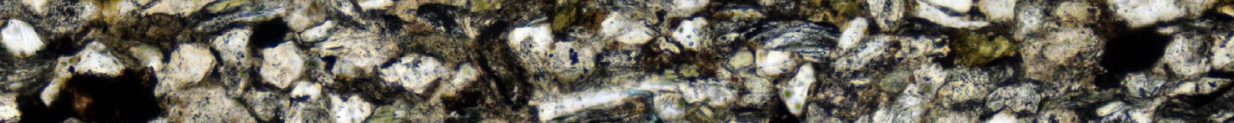

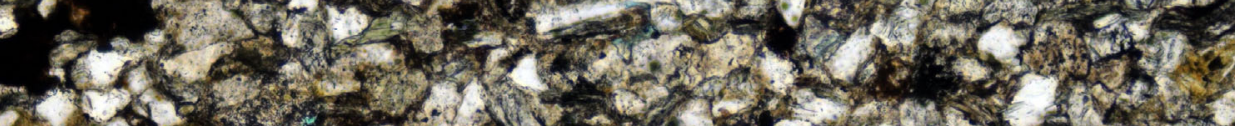

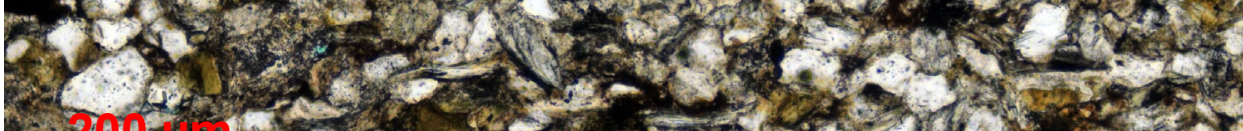

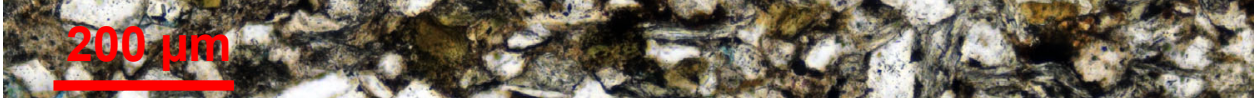

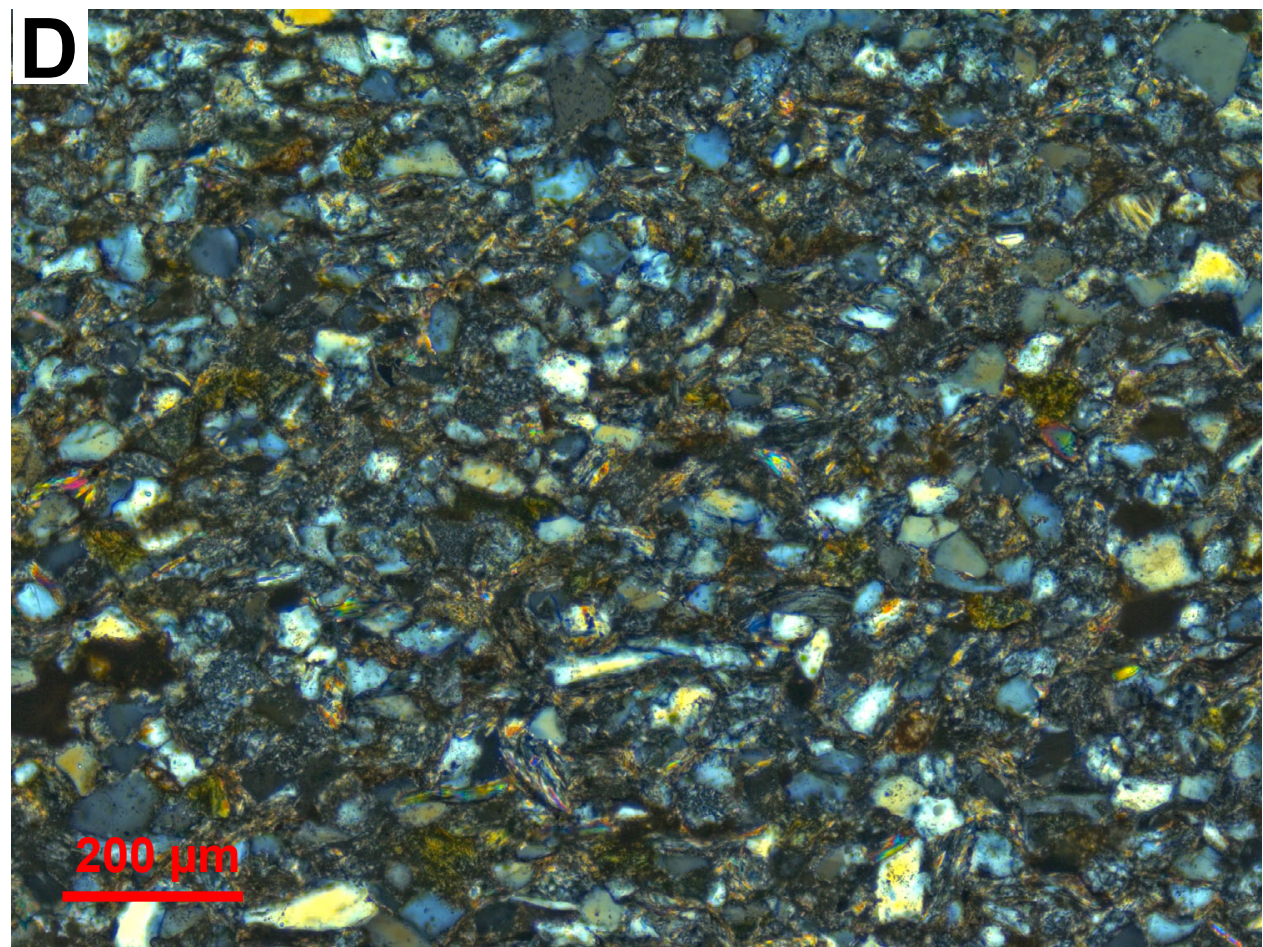


Fig 046 - 00DL022-012.3
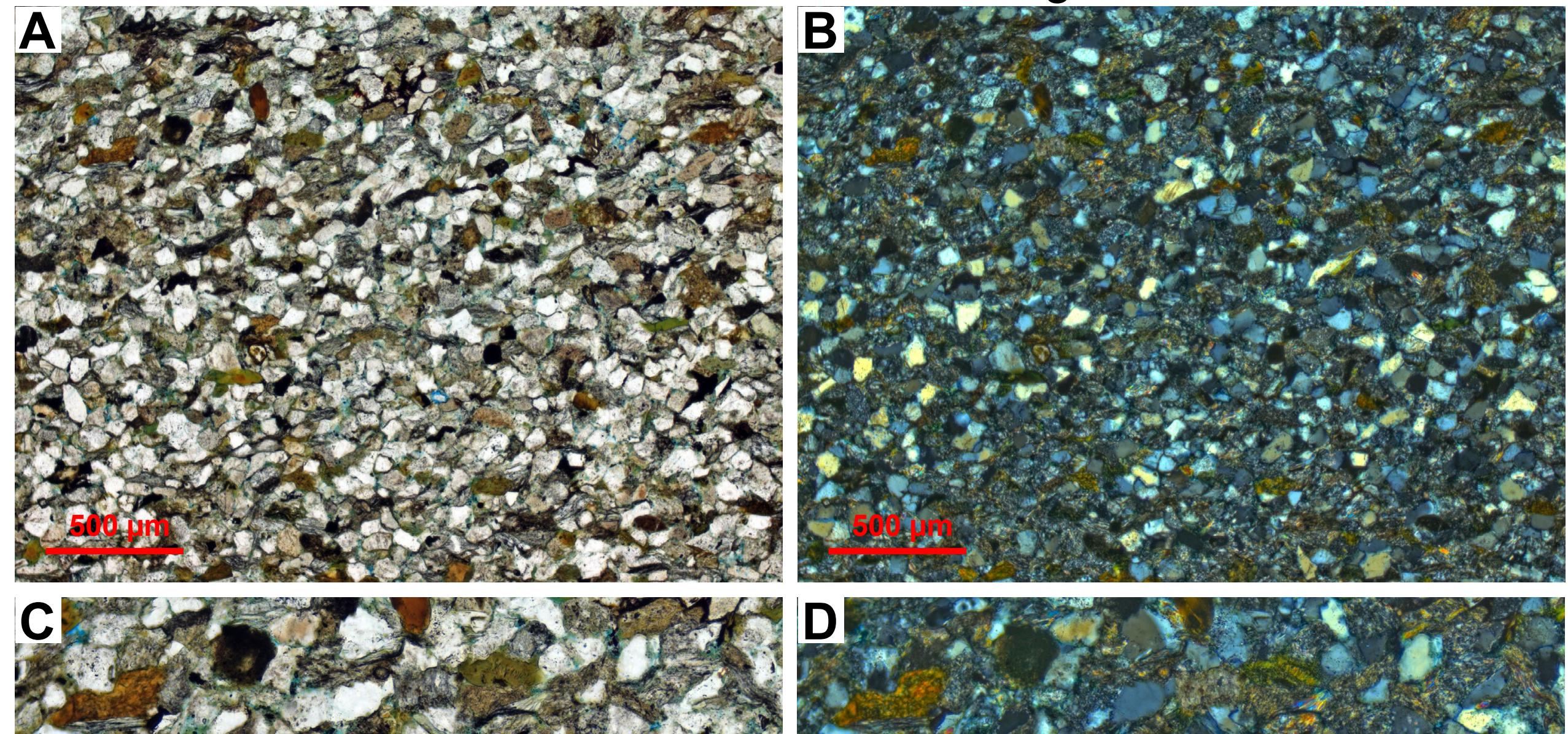

2.20245.

2 . 350 . 12

- o-r.ton - $, 1,2,-210$ T.

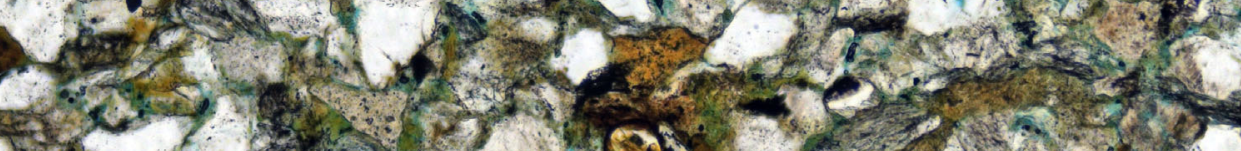

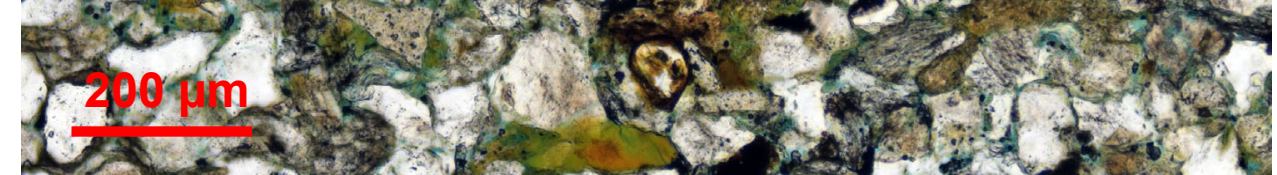

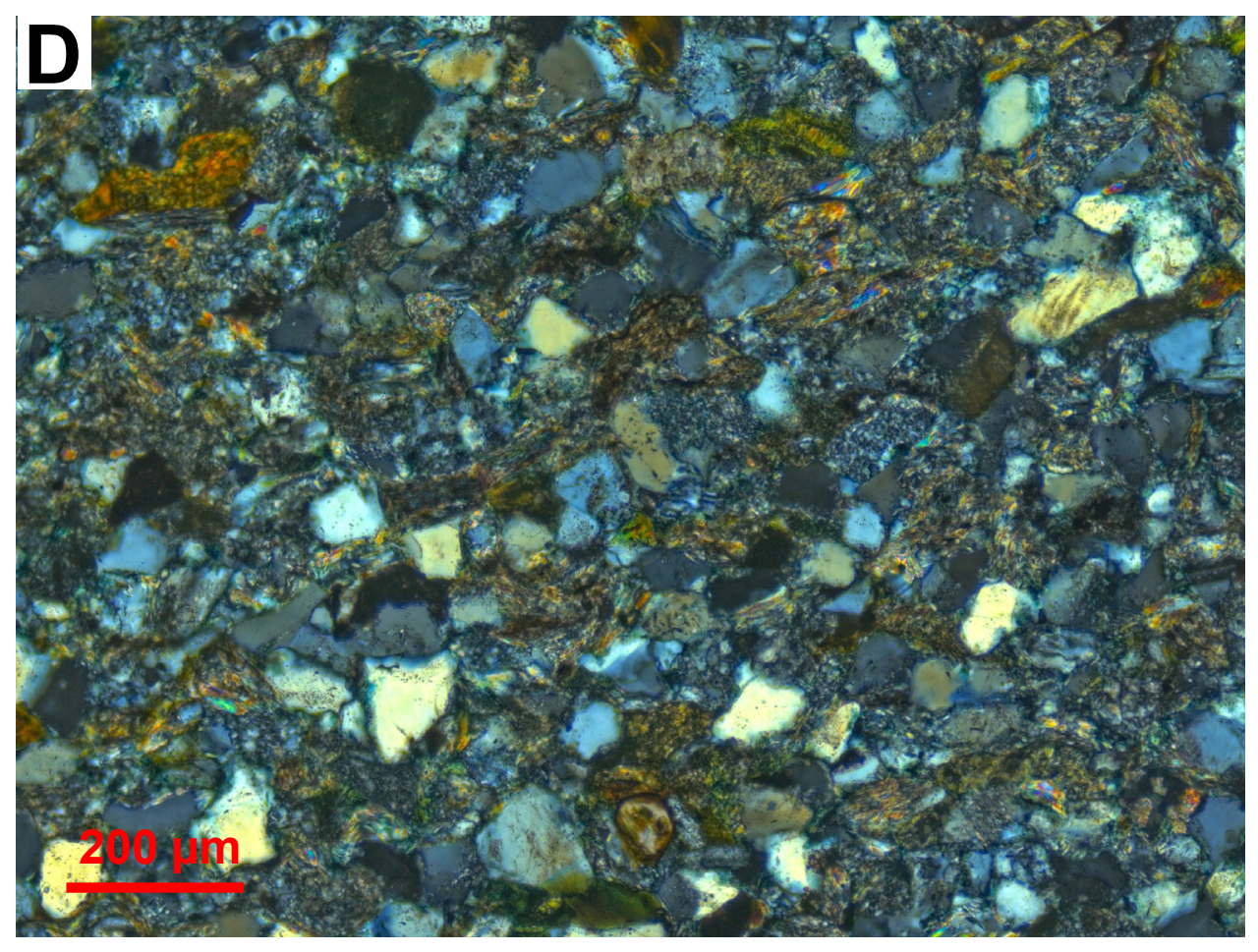




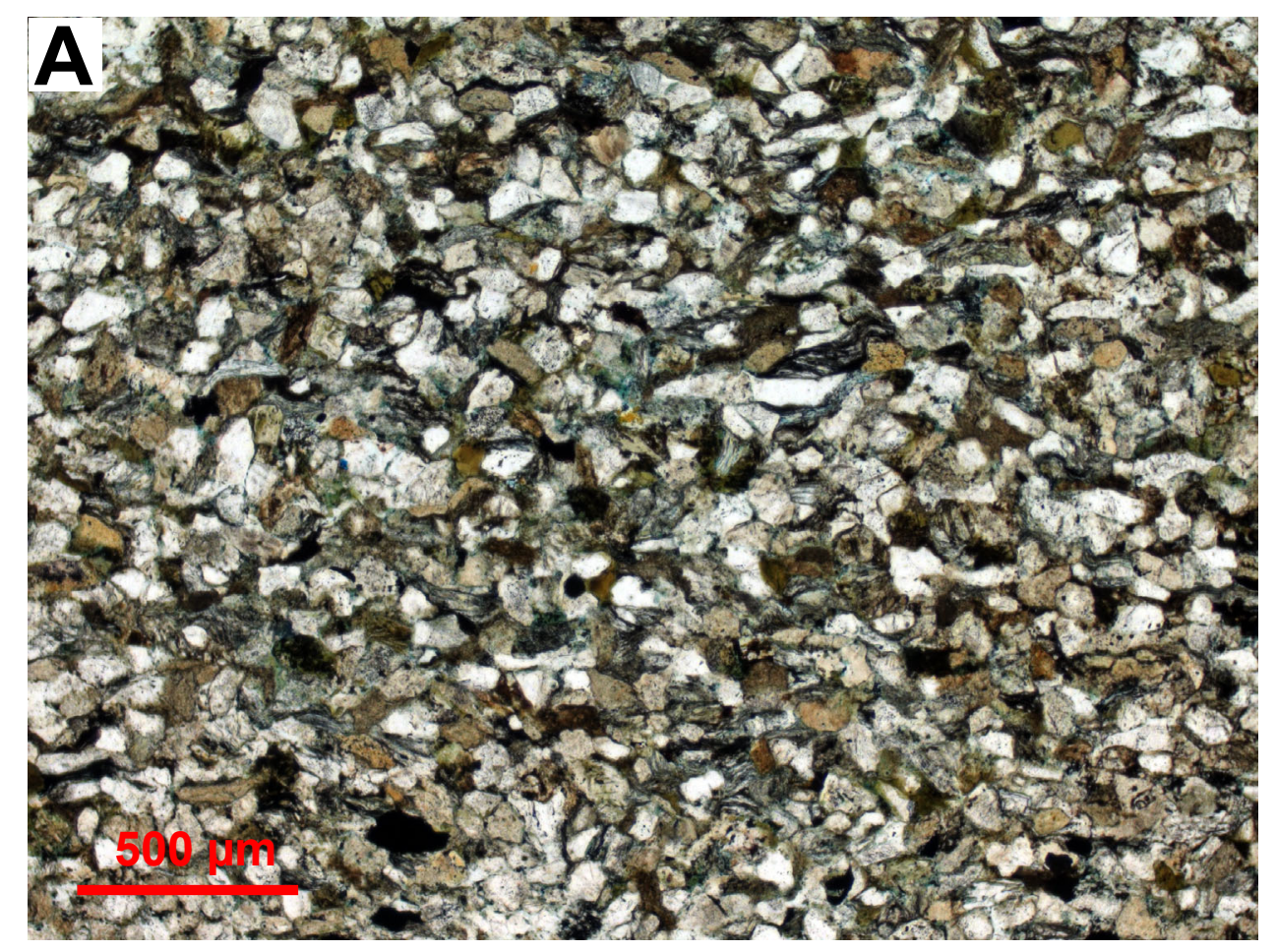

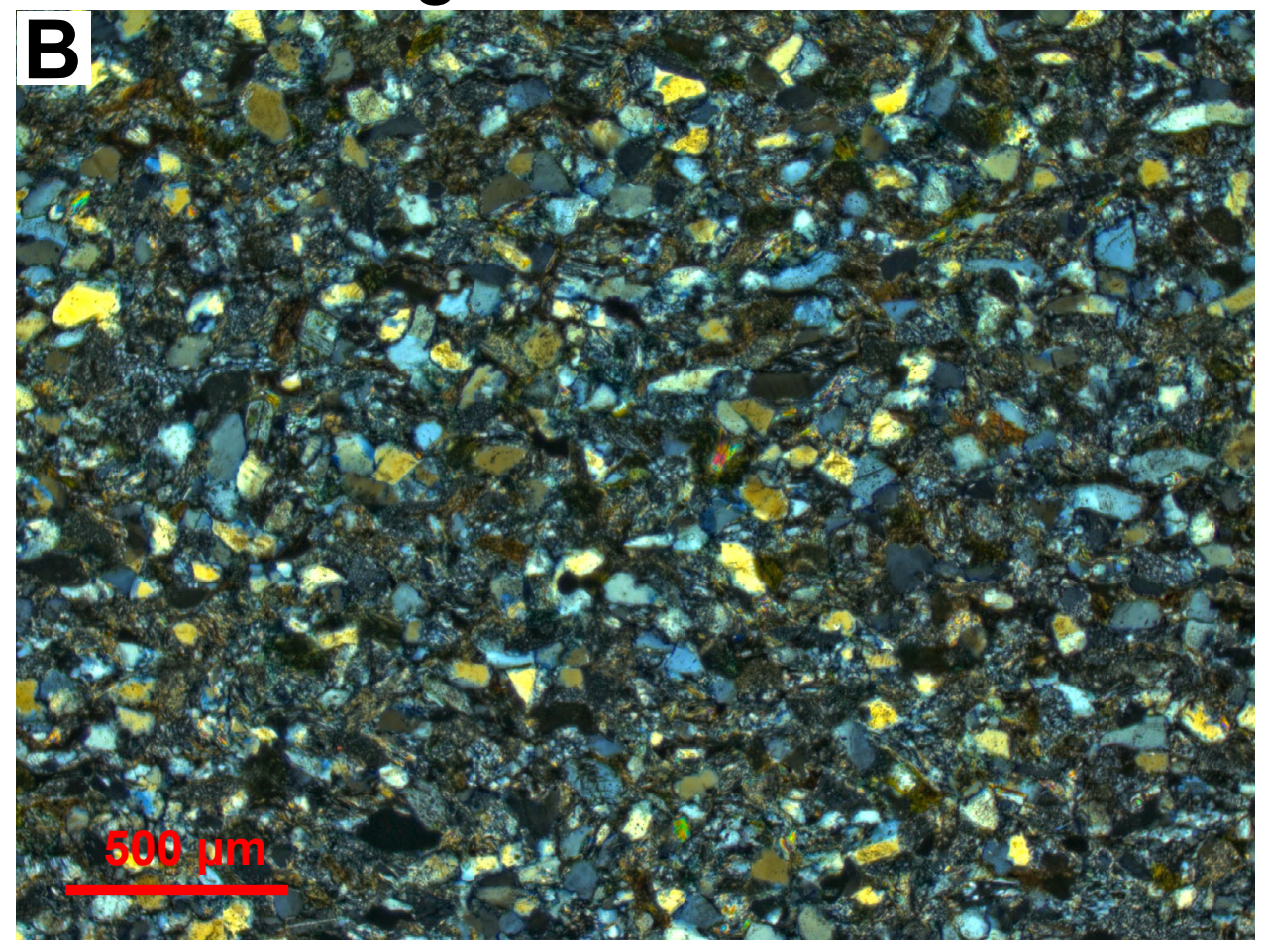
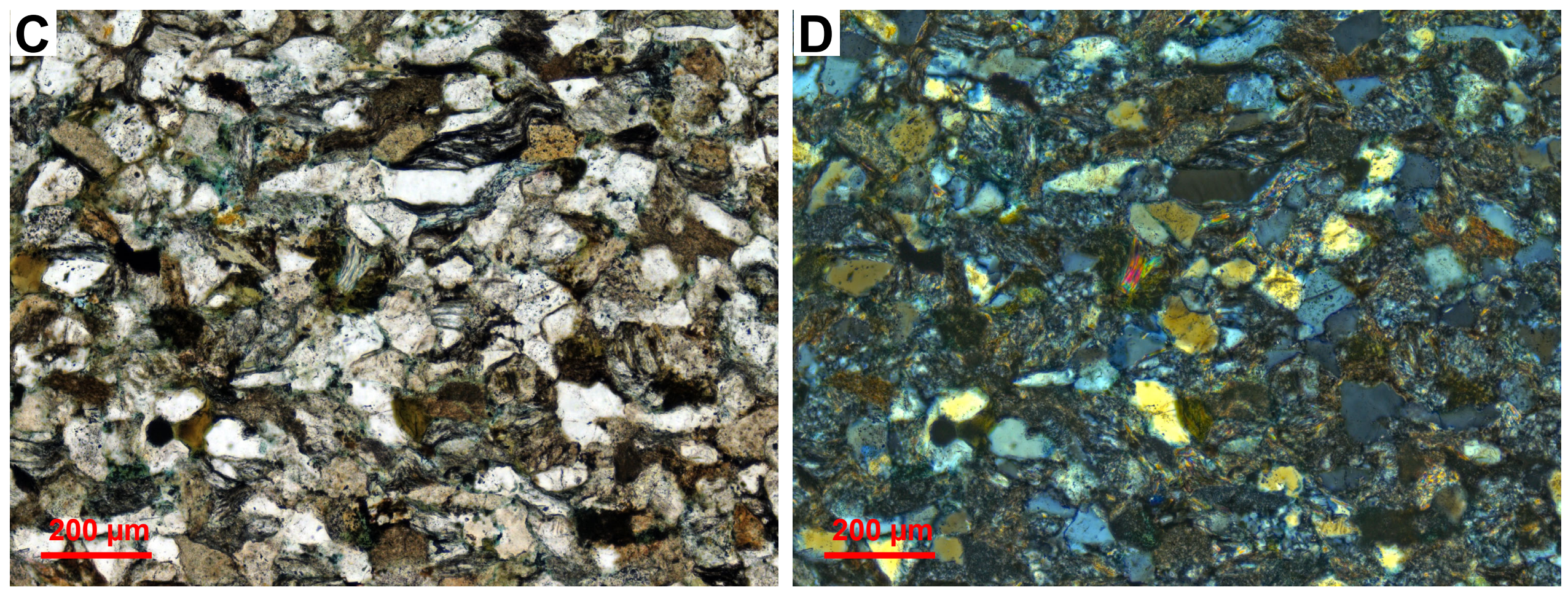
Fig 048 - 00DL022-038.0

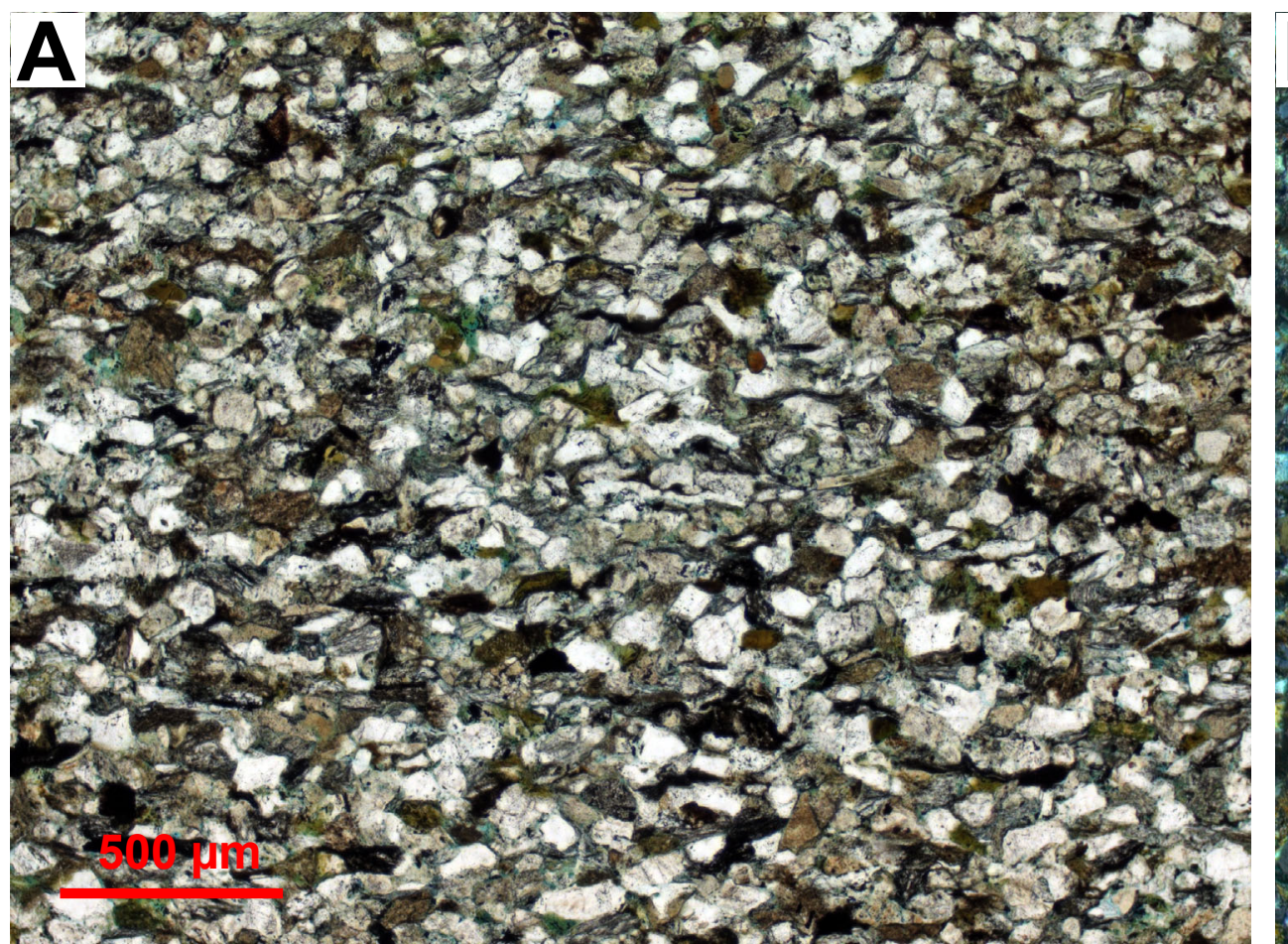

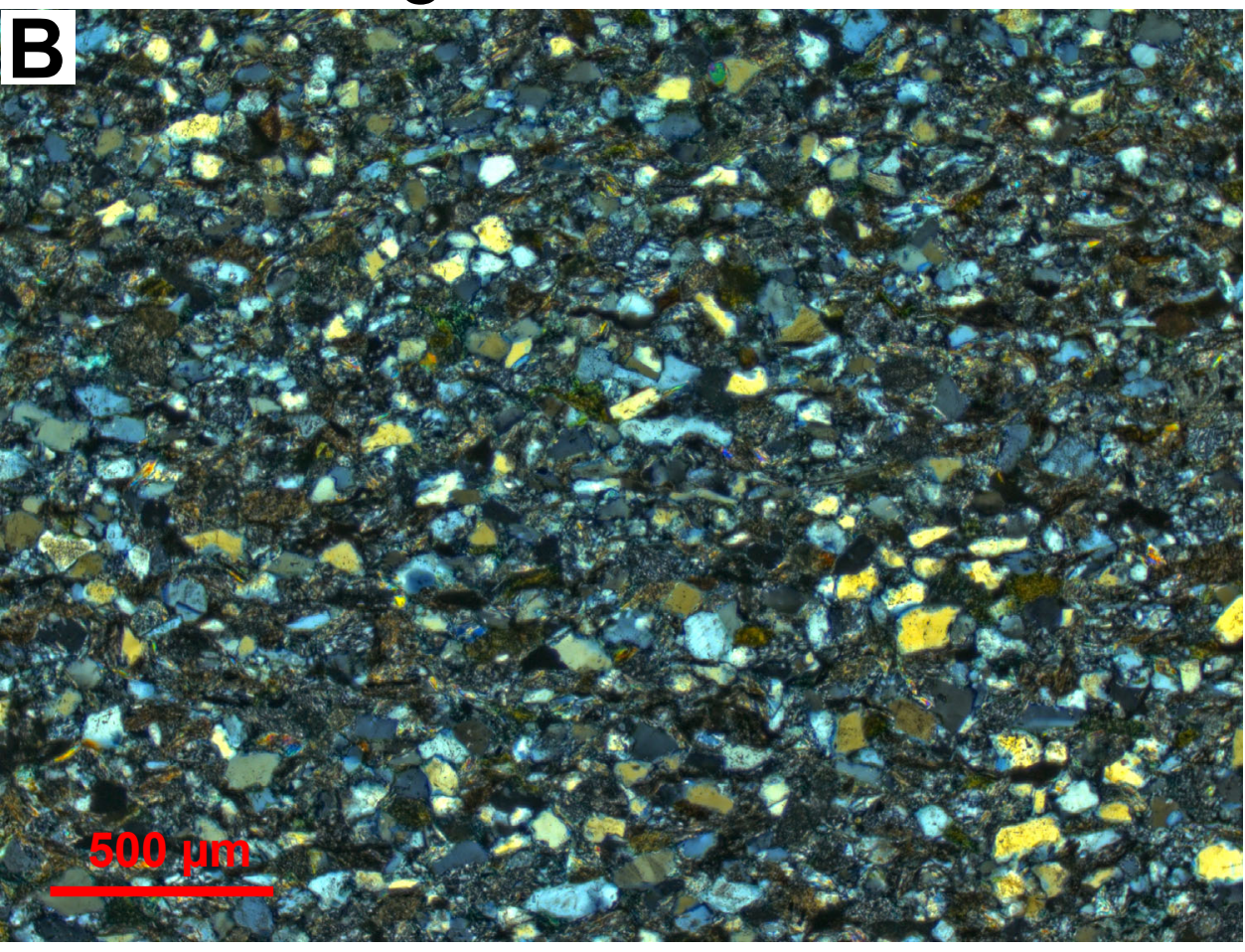

crus

$2.15,20$

- 50

- 120215 20 45013 - r r o

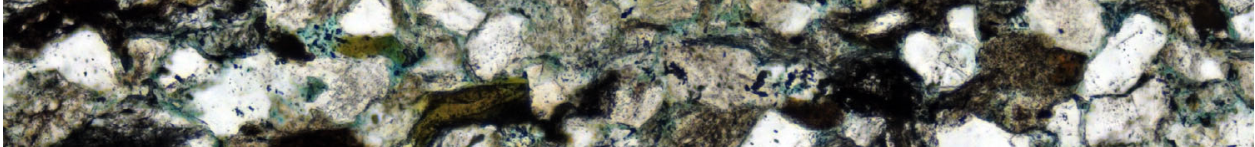
- a er 402 1.

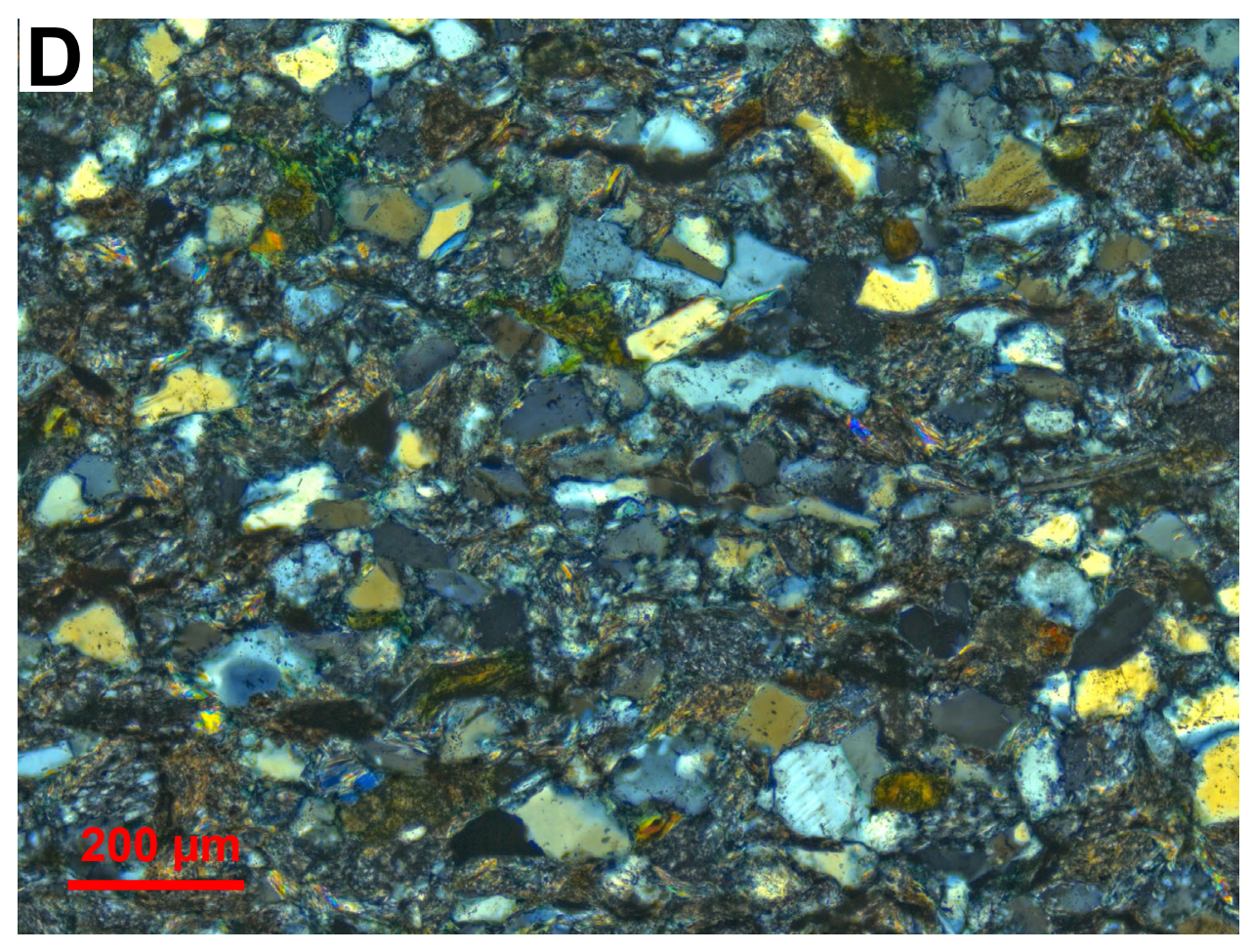


Fig 049 - 00DL022-046.0

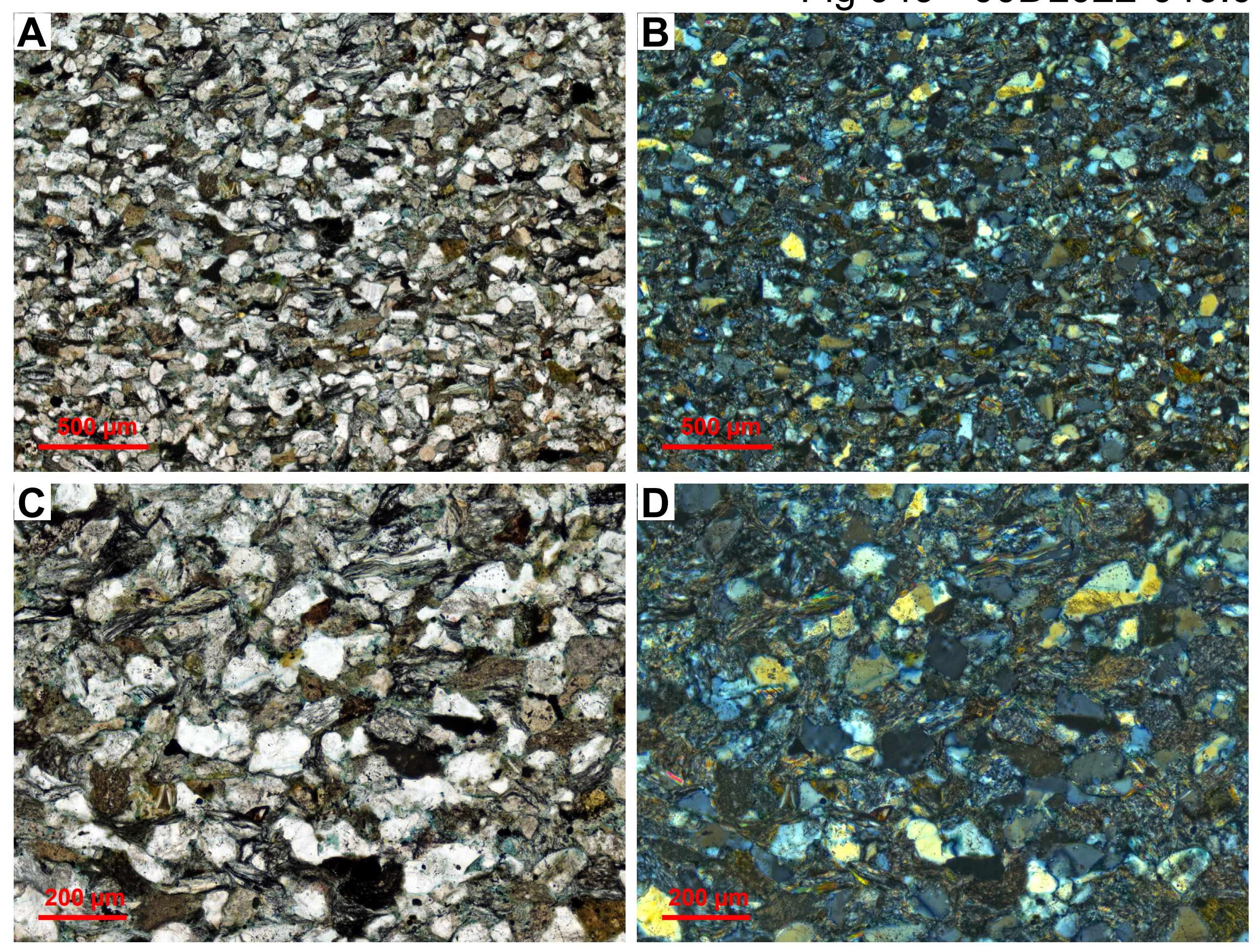


Fig 050 - 00DL022-077.5

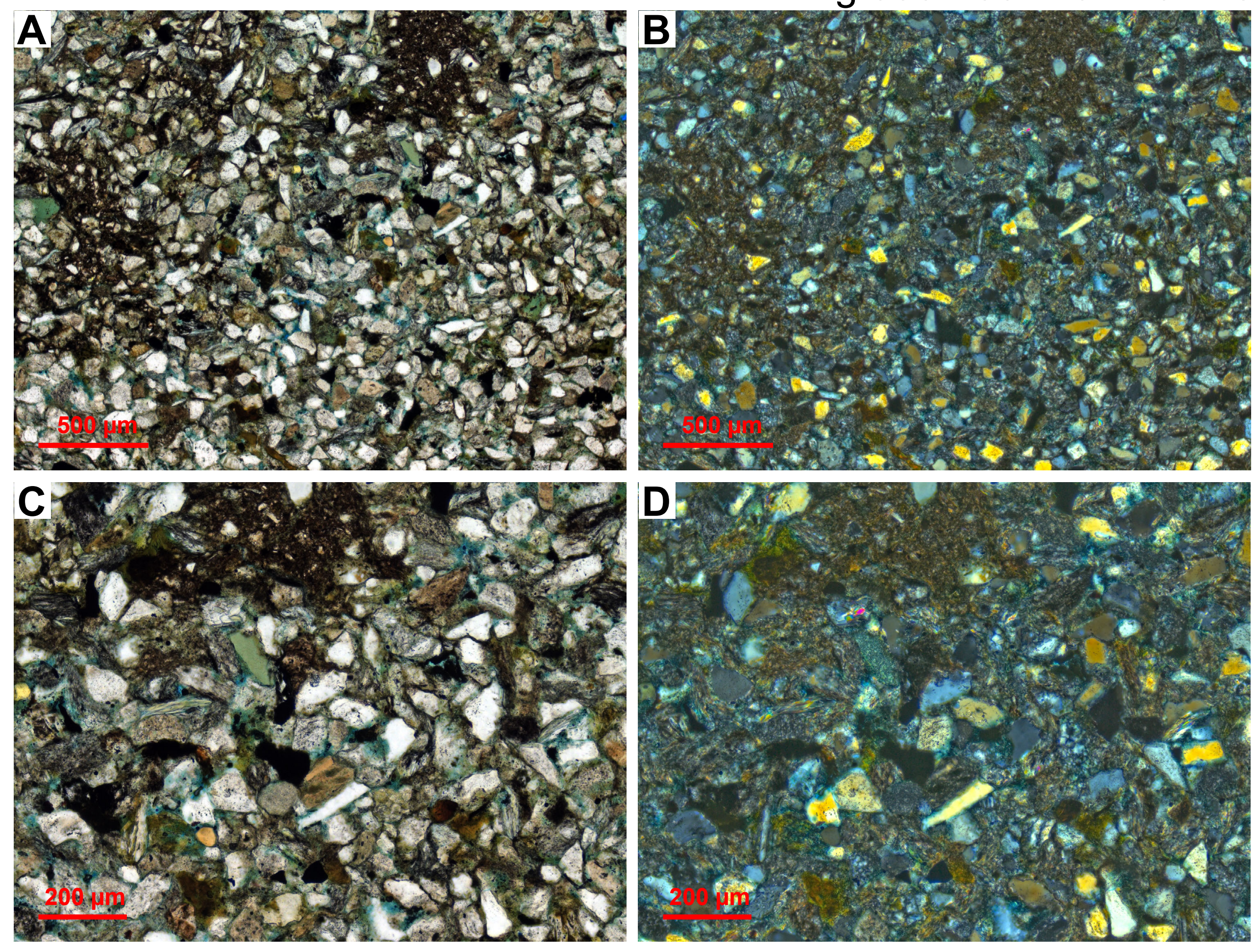


Fig 051 - 00DL022-088.4
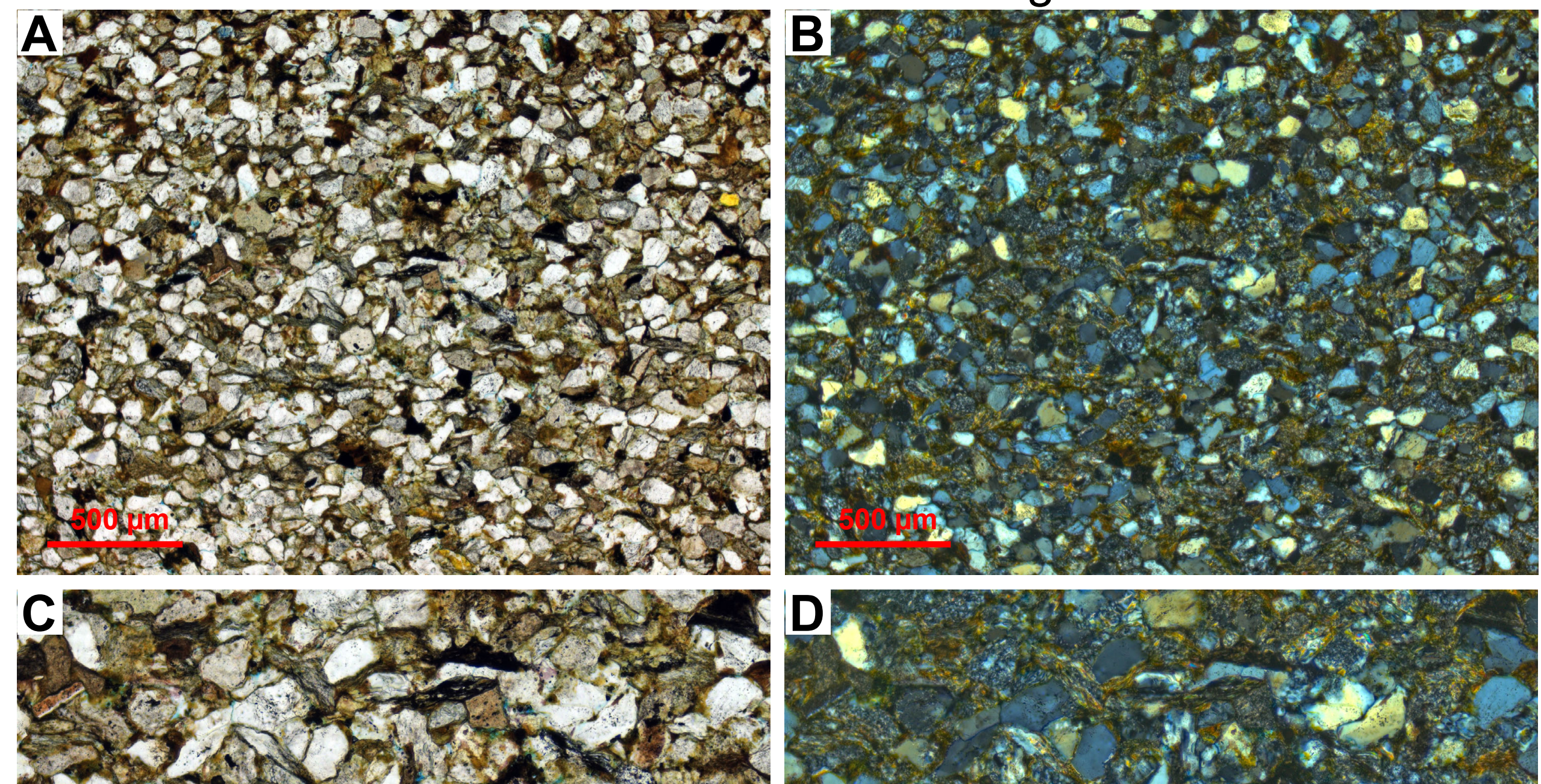

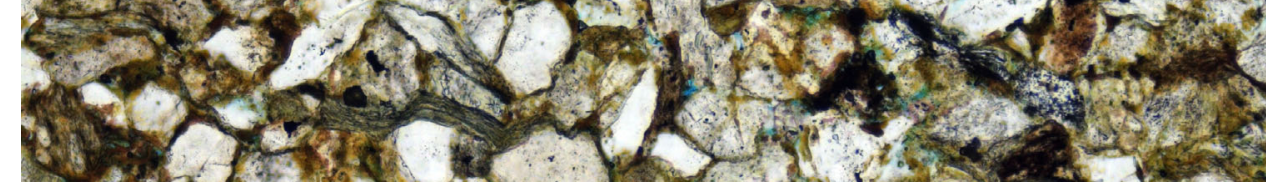
100,20
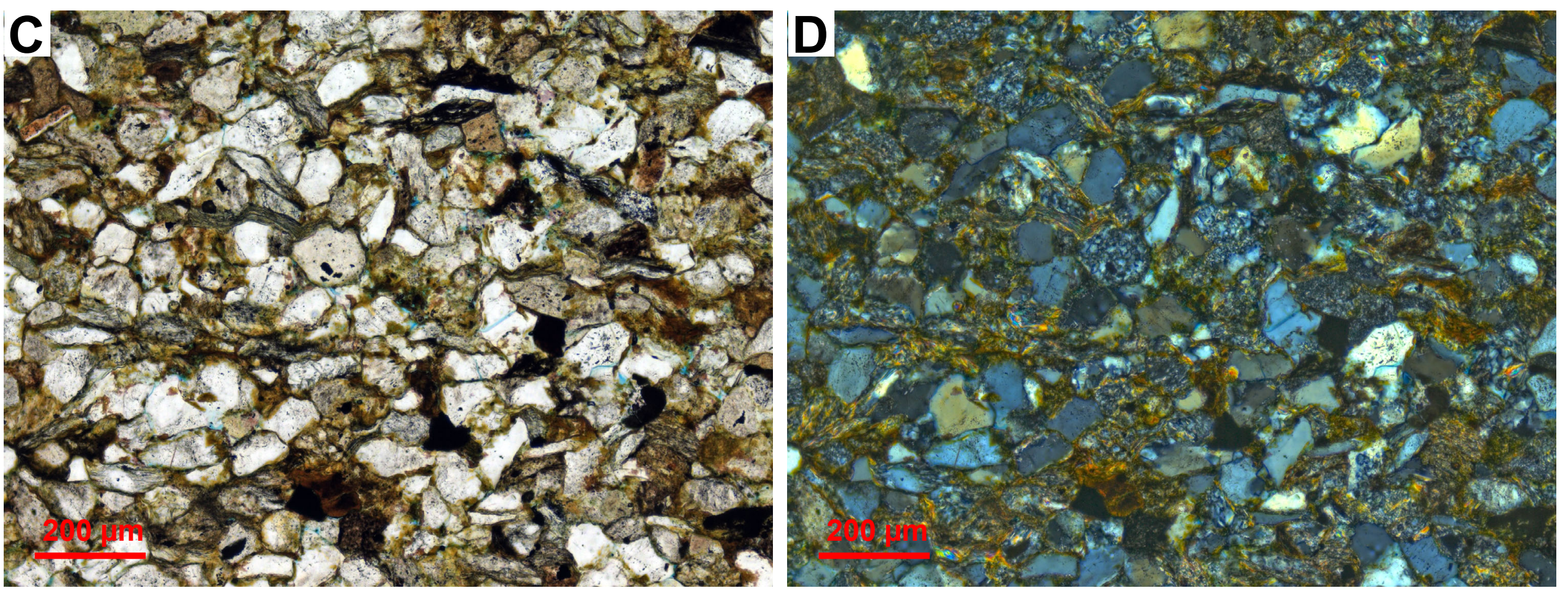
Fig 052 - 00DL022-100.8
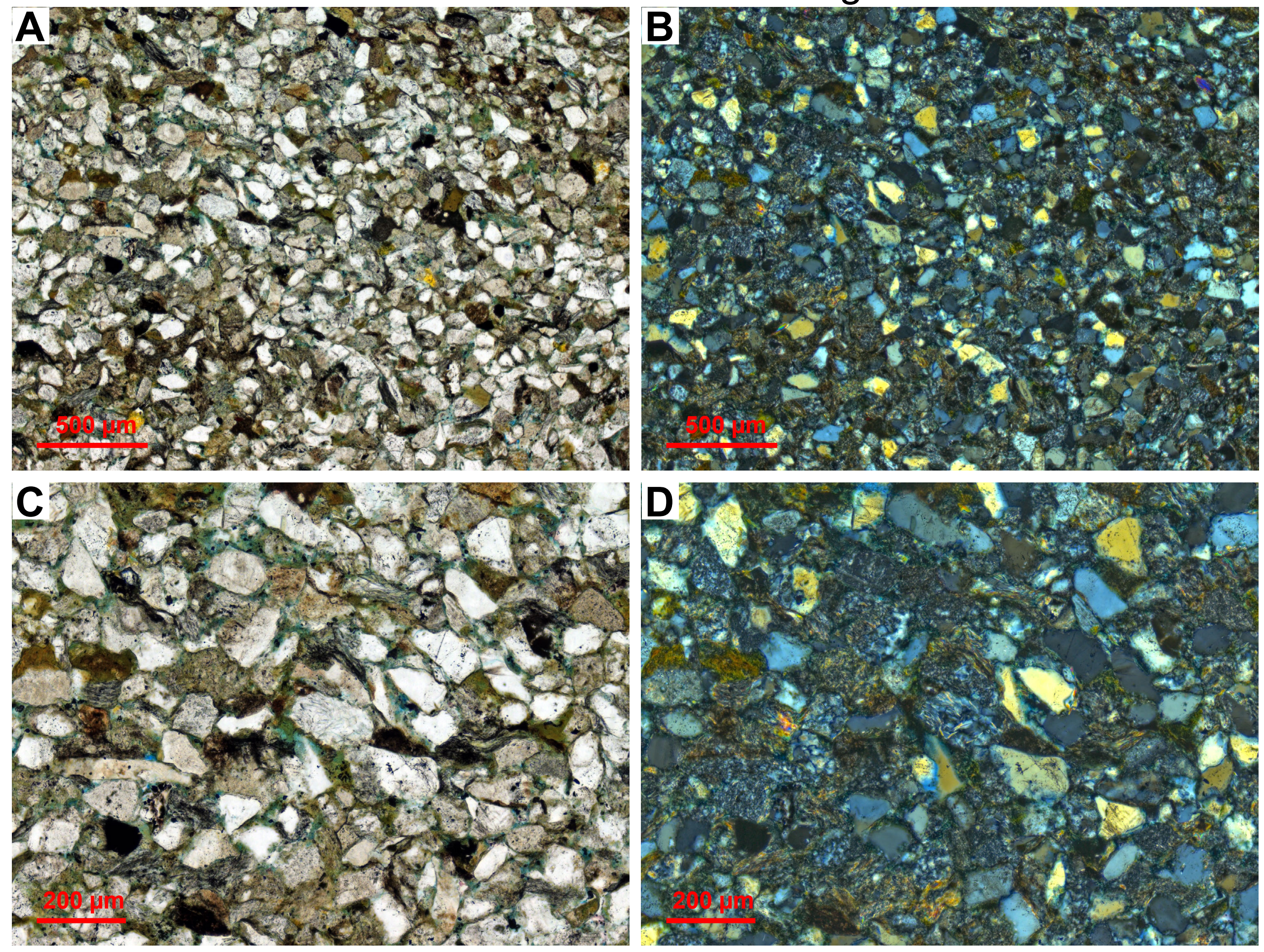
Fig 053 - 00DL022-110.0

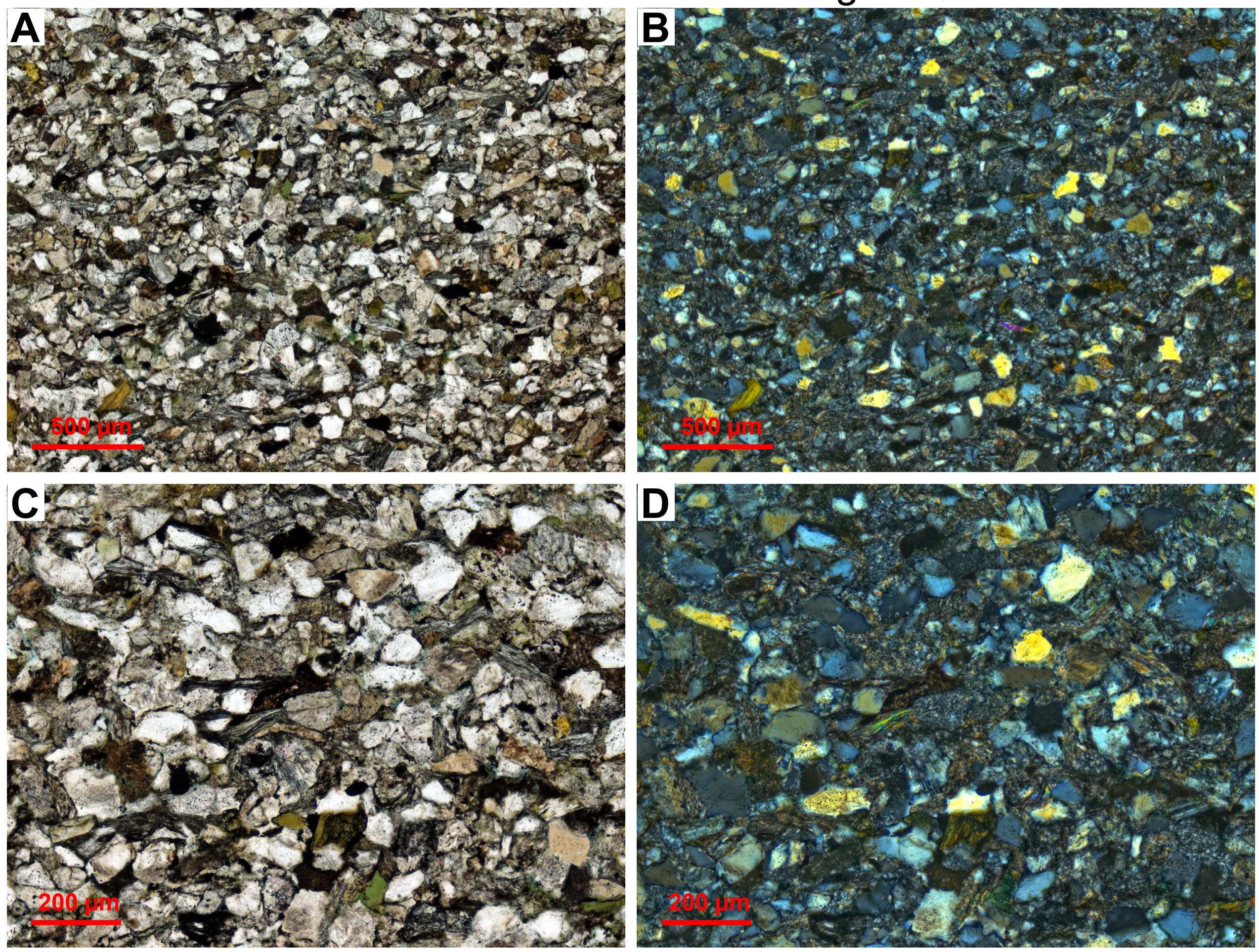


Fig 054 - 00DL022-115.4
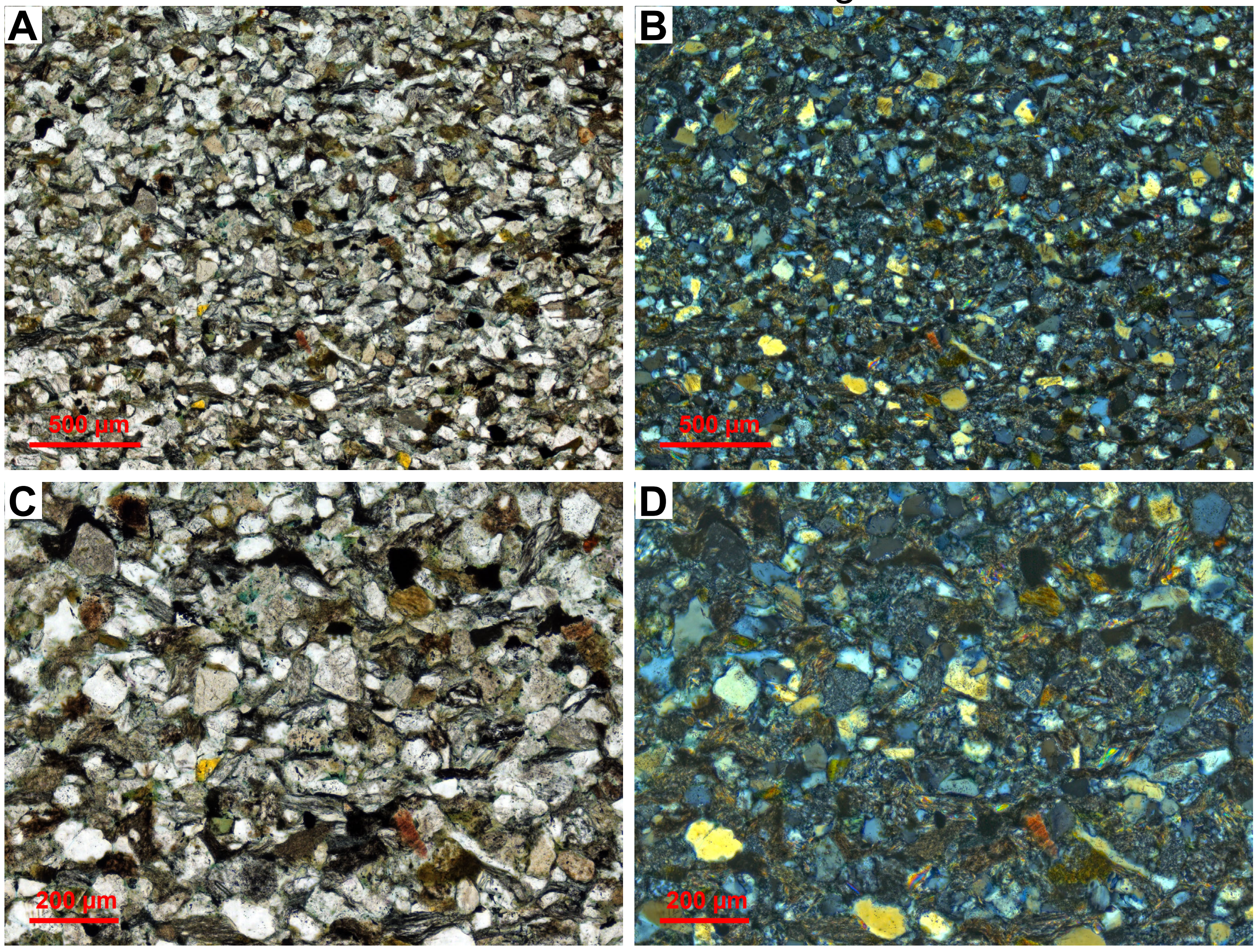
Fig 055 - 00DL022-132.0

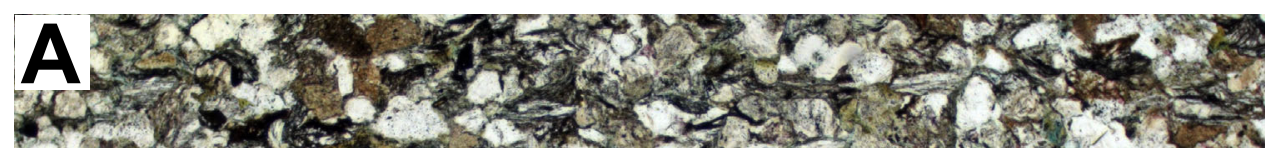

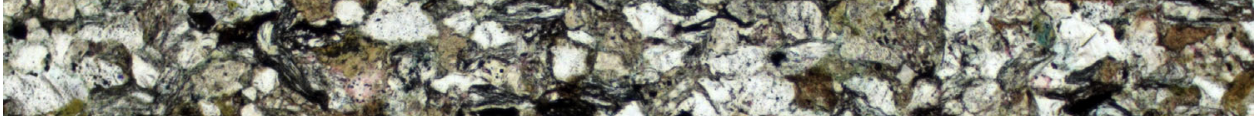

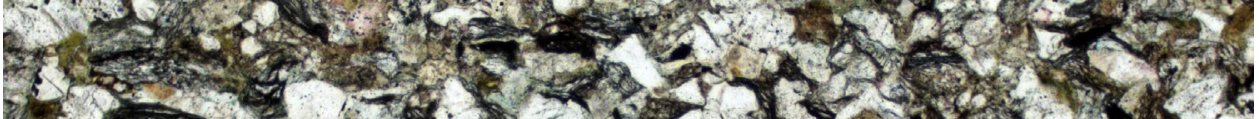

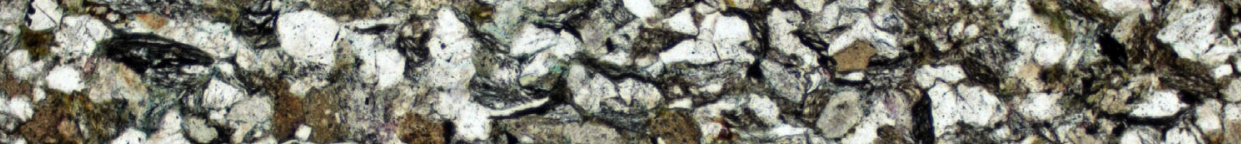

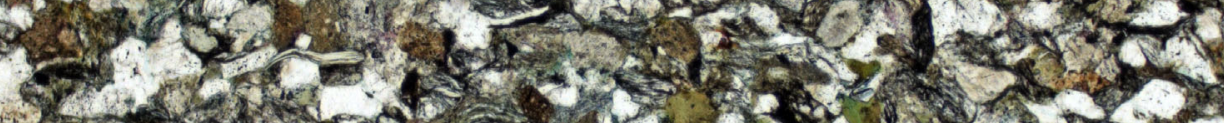

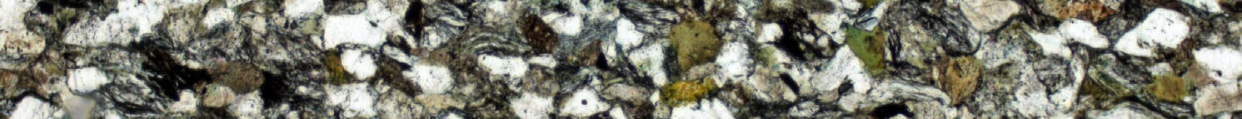

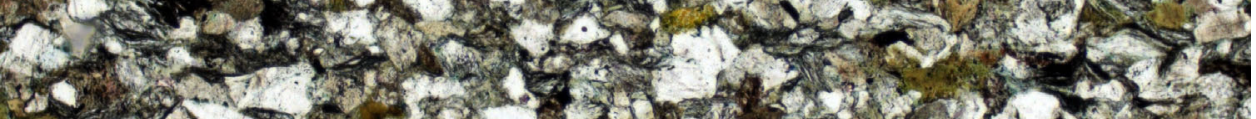

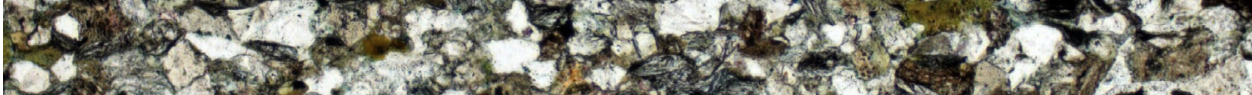

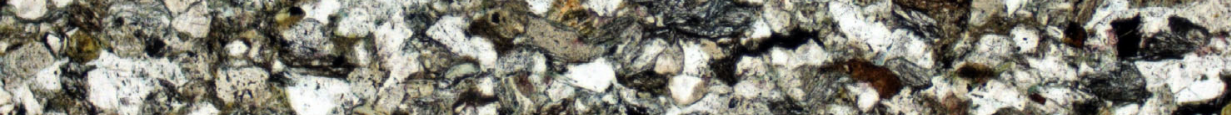

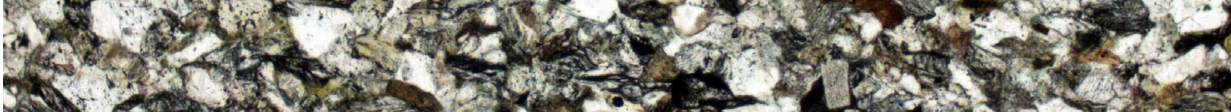

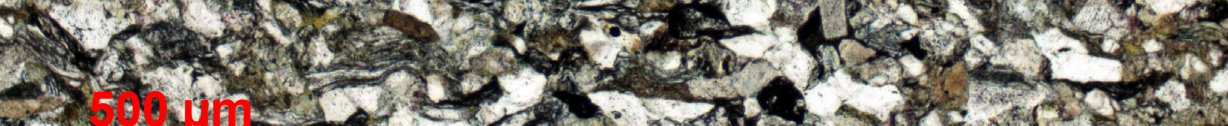

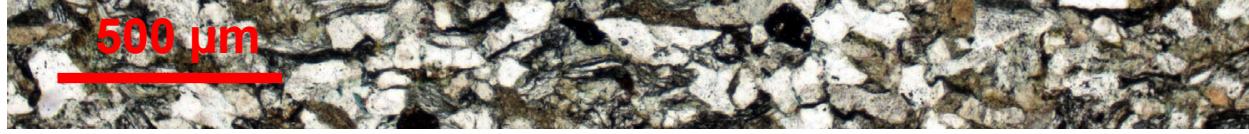

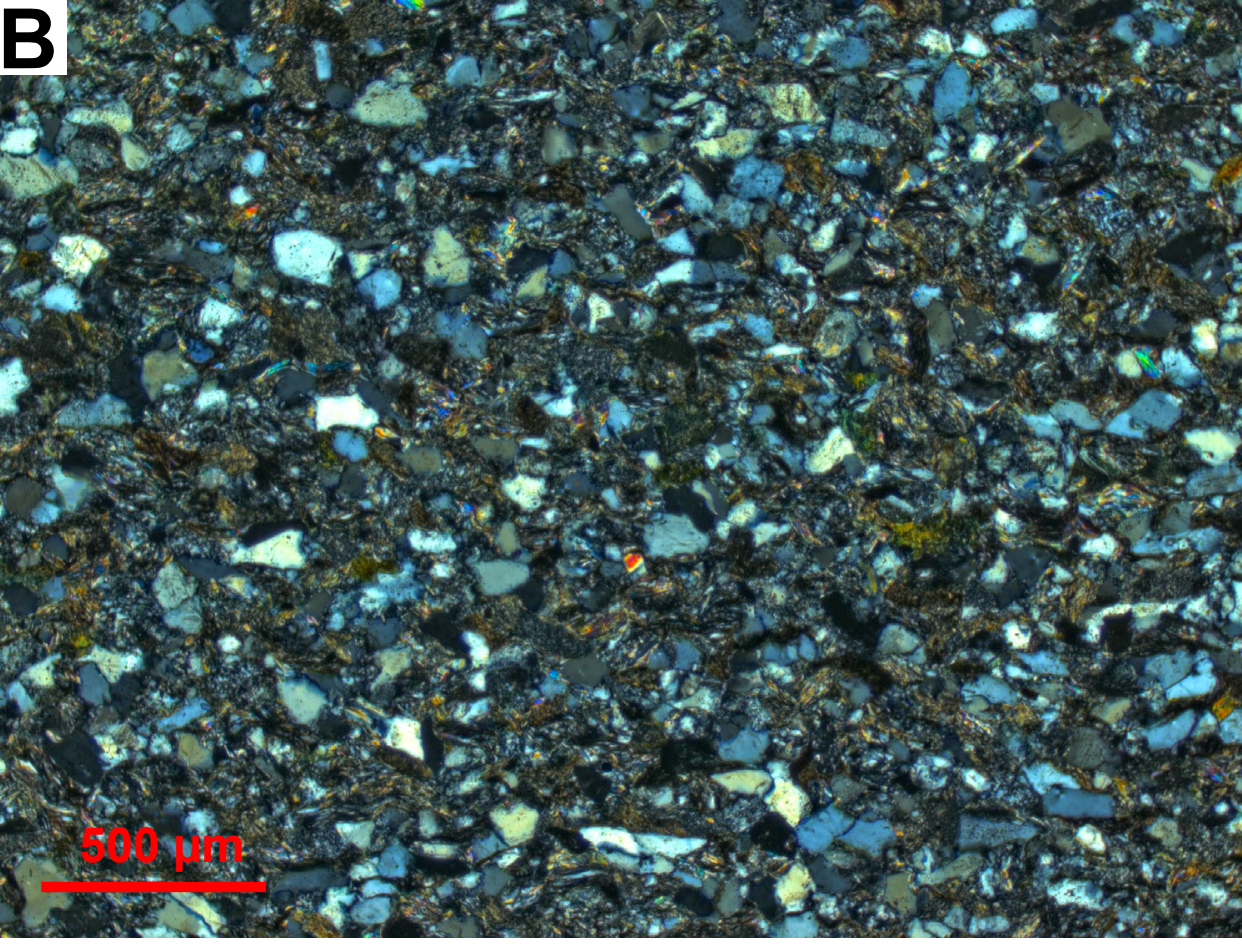

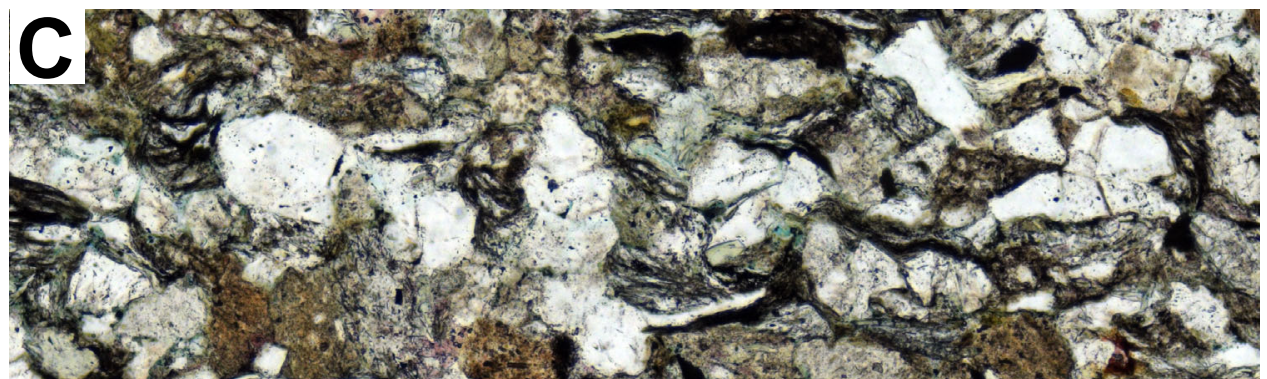

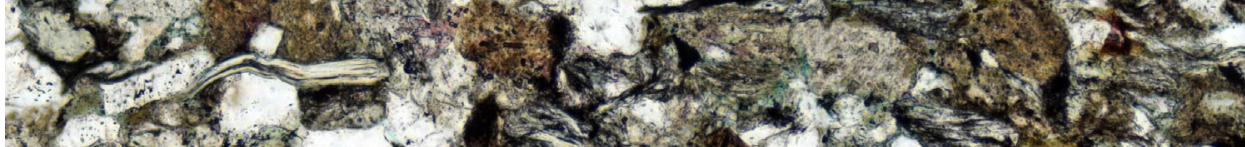

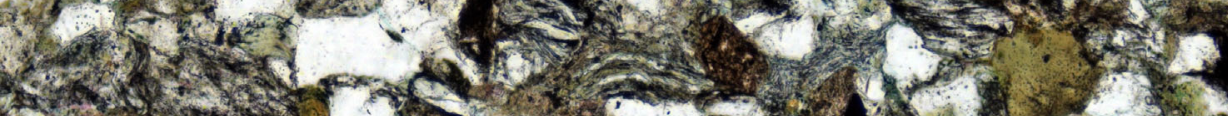

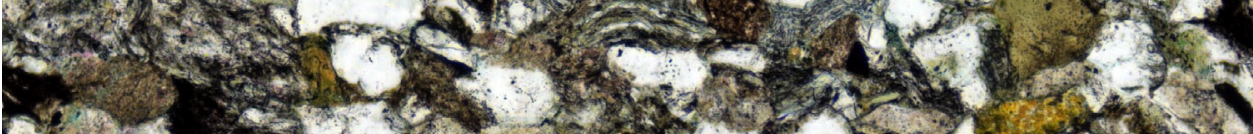

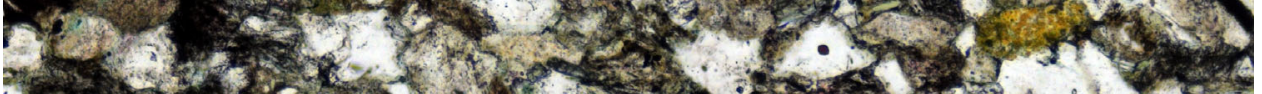

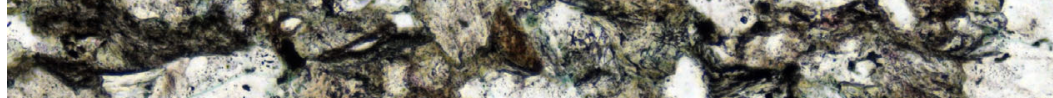

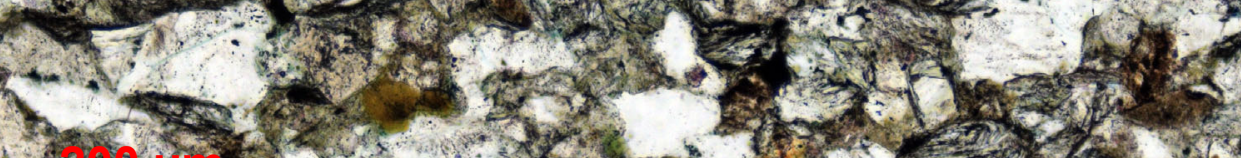

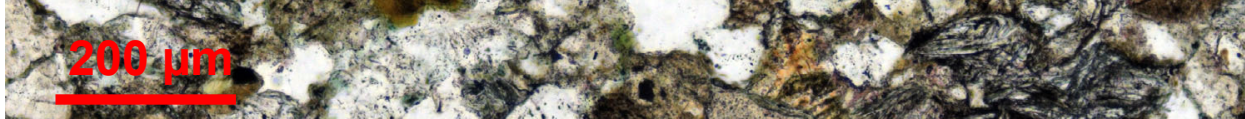

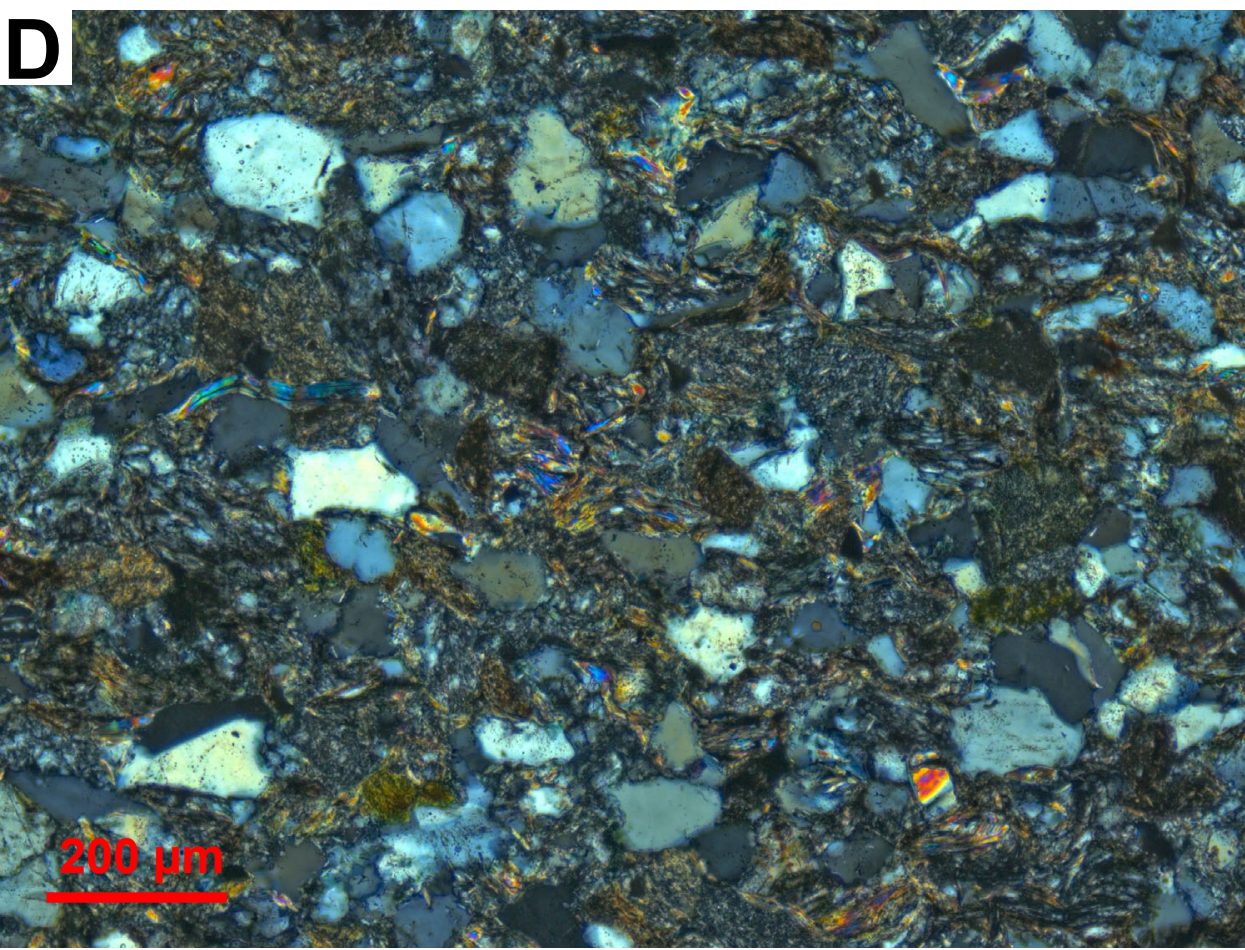


Fig 056 - 00DL022-134.5

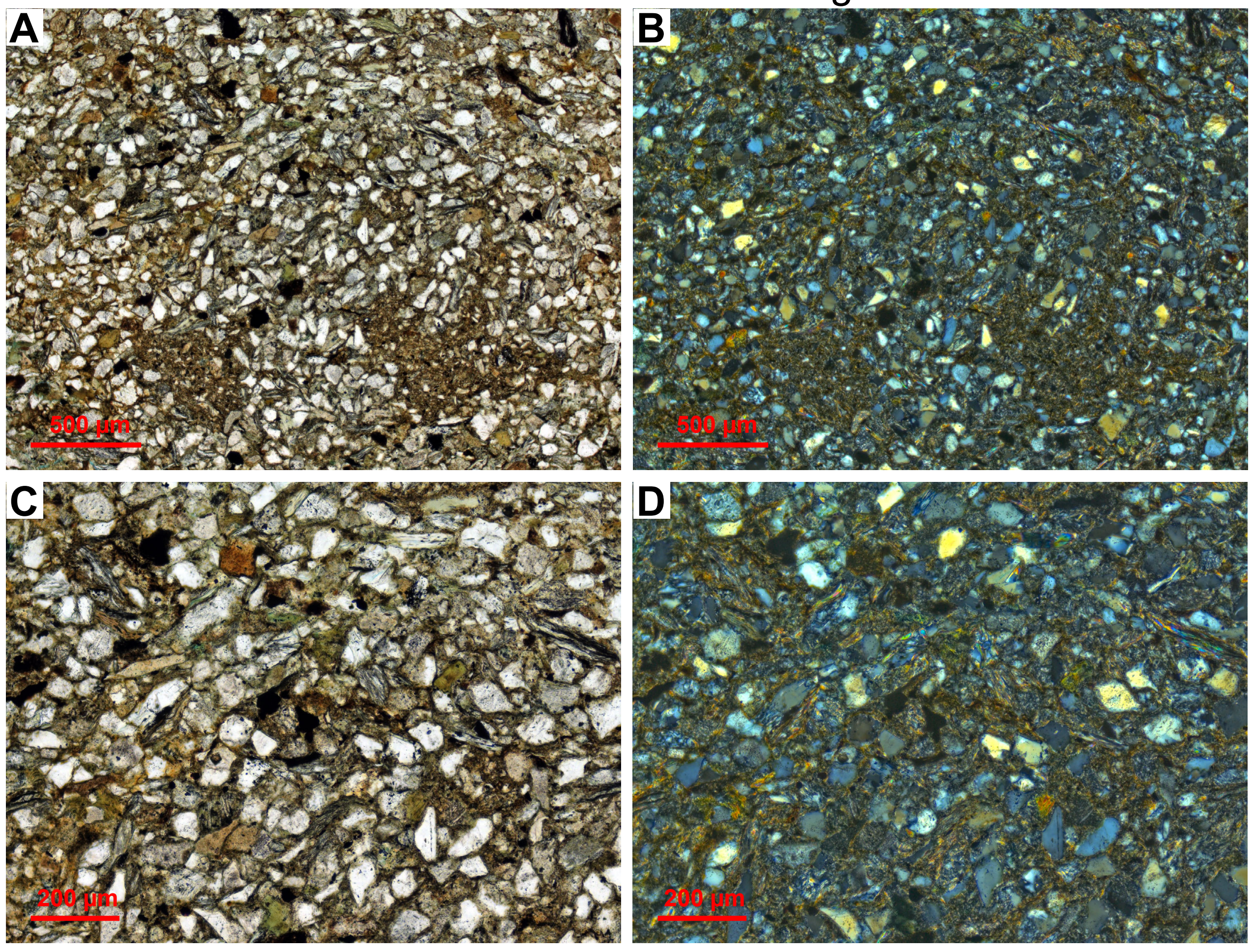


Fig 057 - 00DL022-157.5

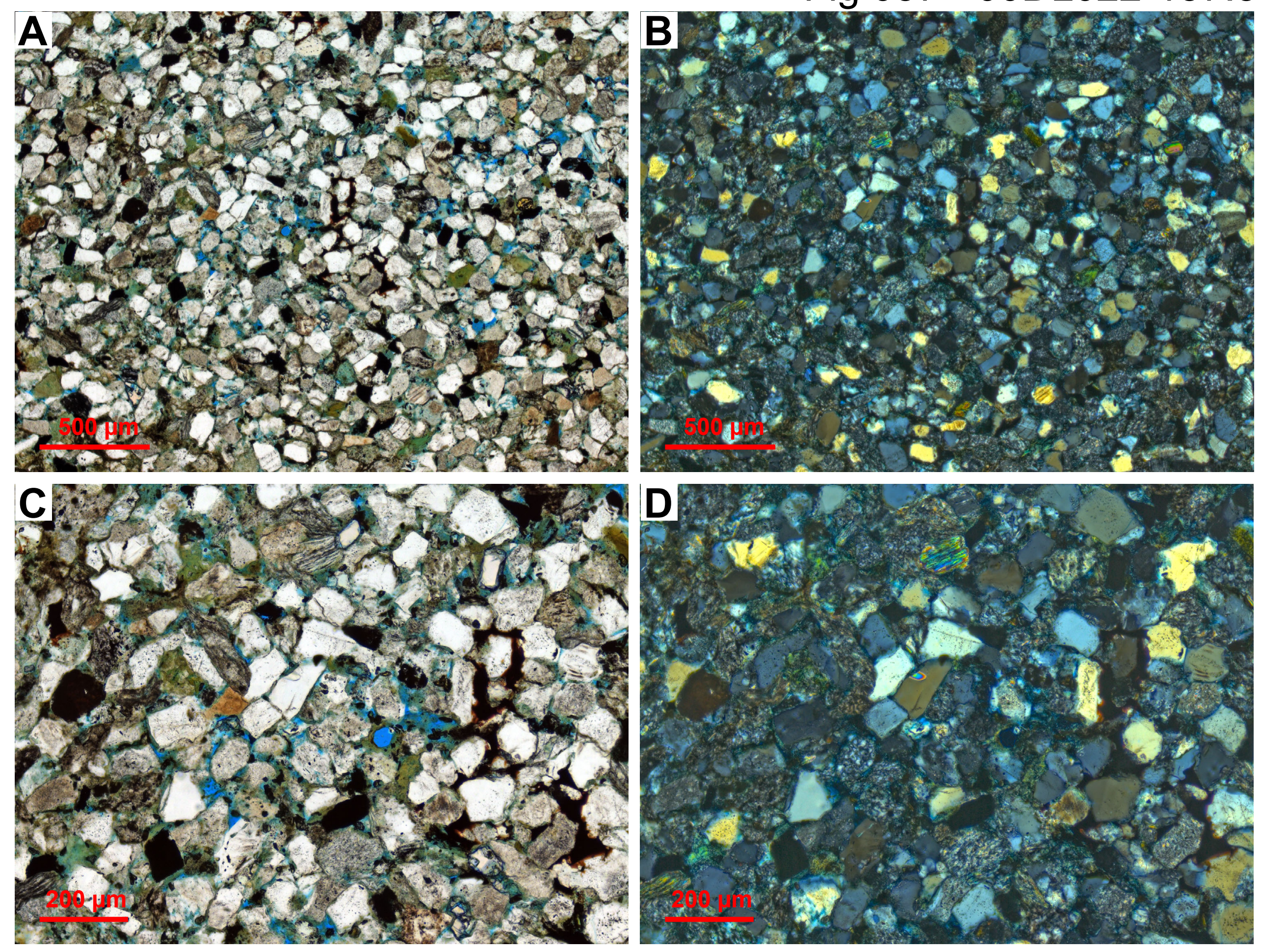


Fig 058- 000DL022-194.7

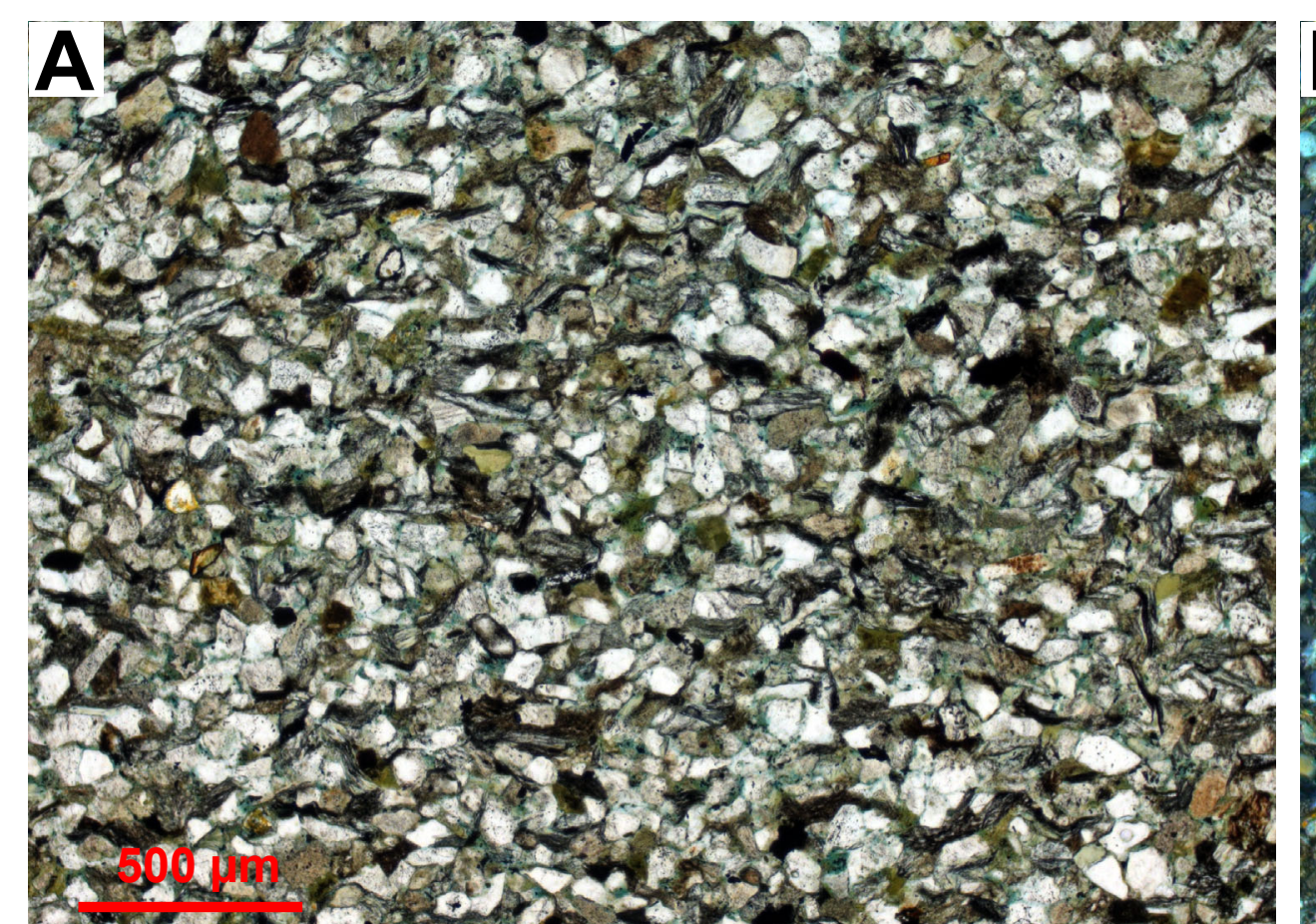

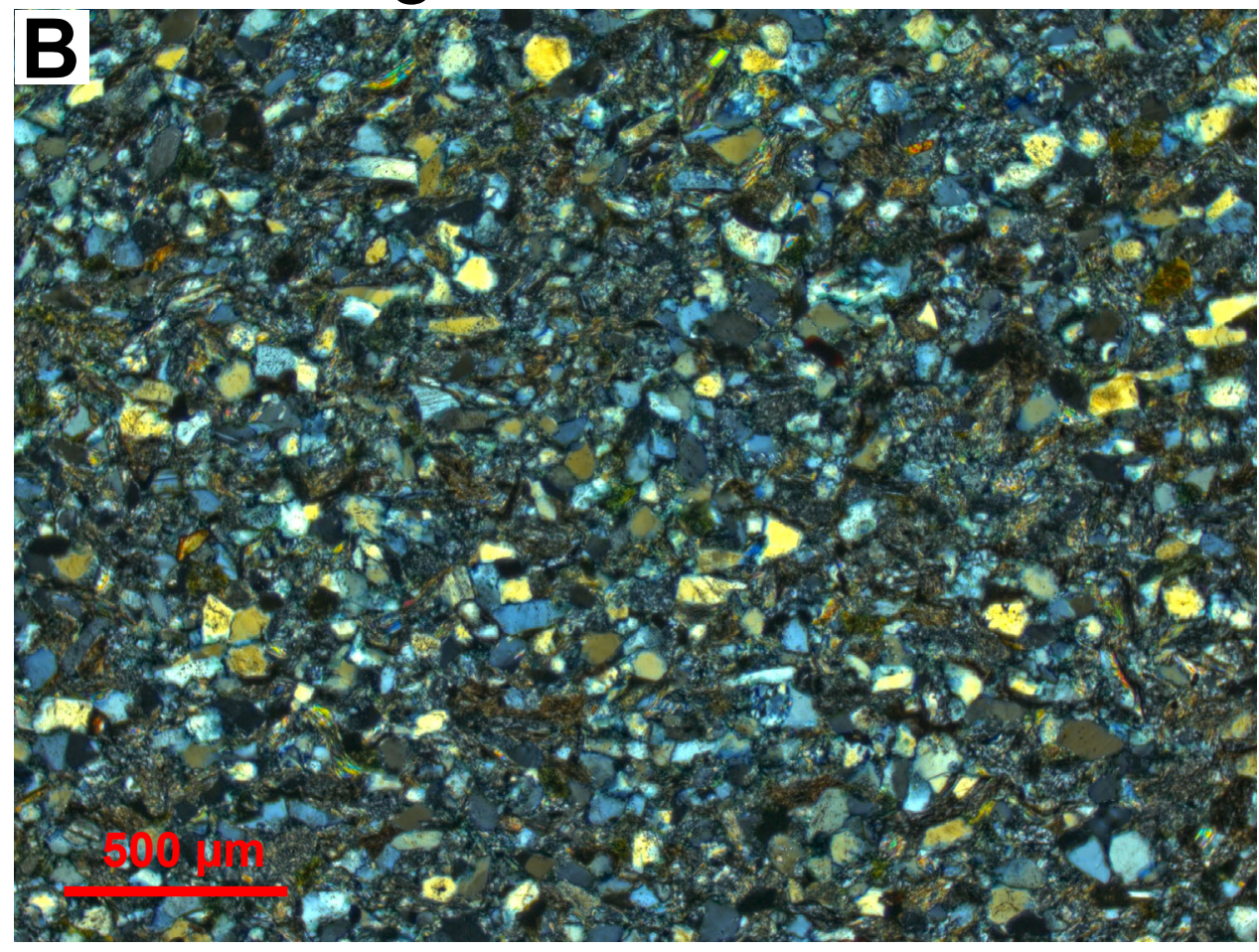

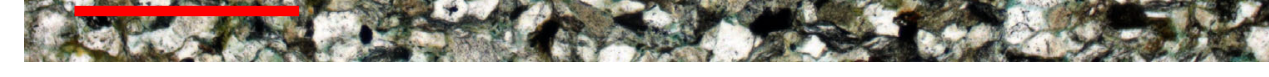

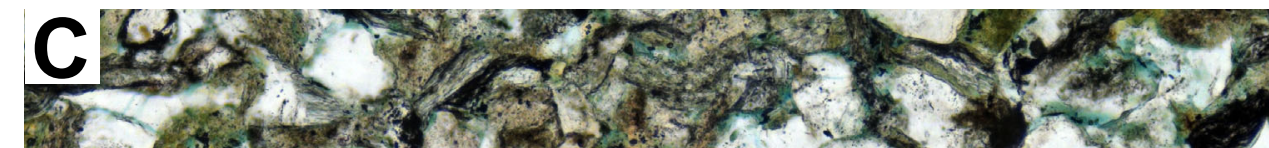
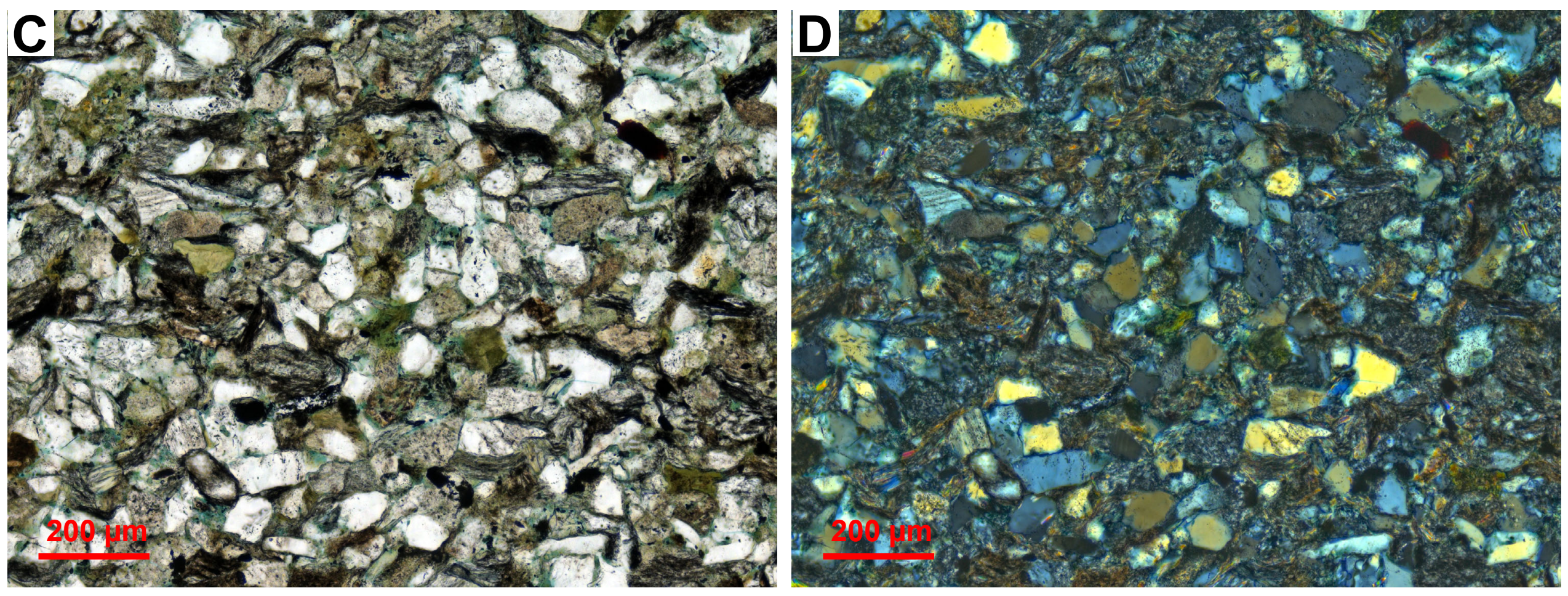
Fig 059 - 00DL022-203.0

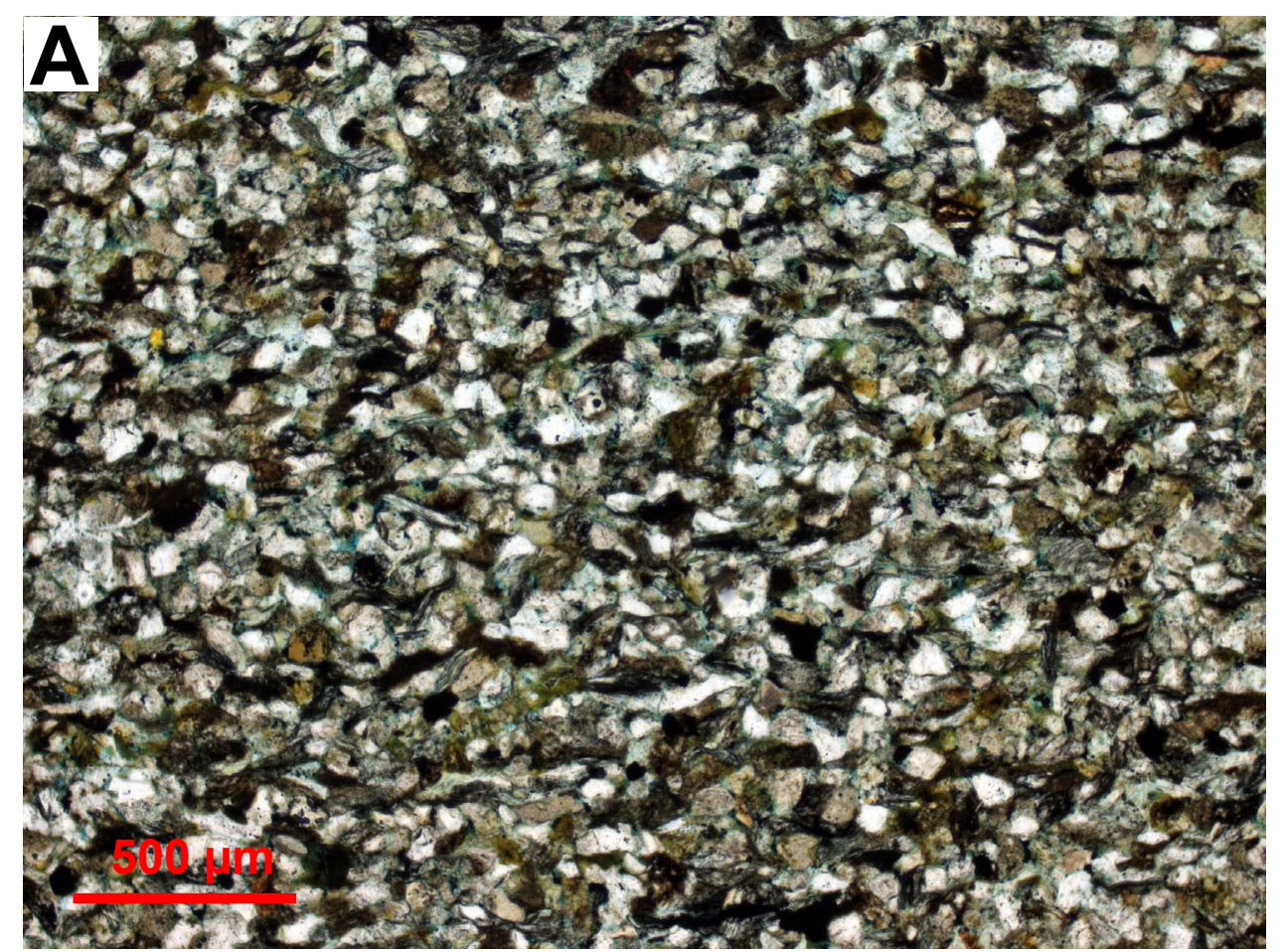
B $x+2$ is $-4,-20$

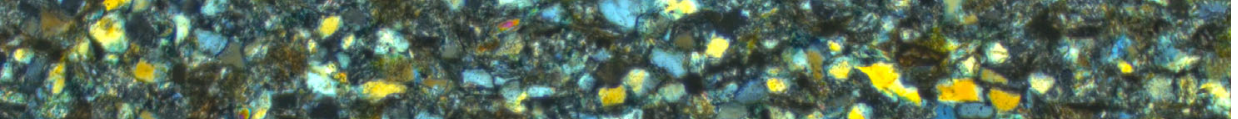

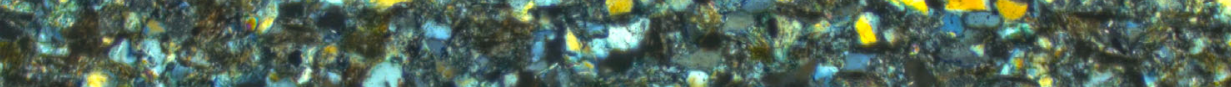

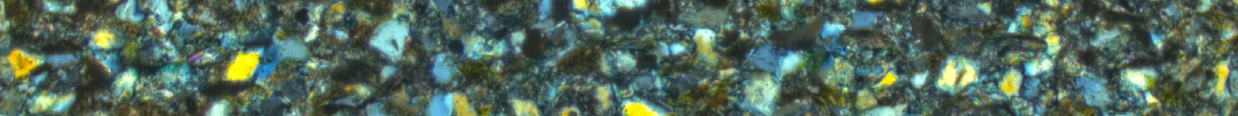

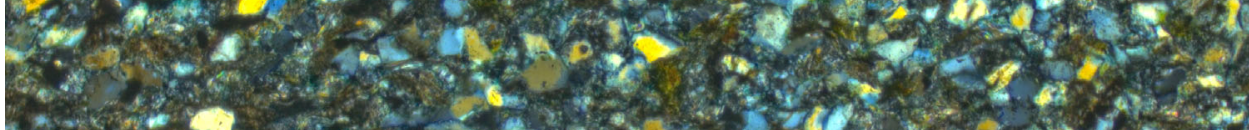

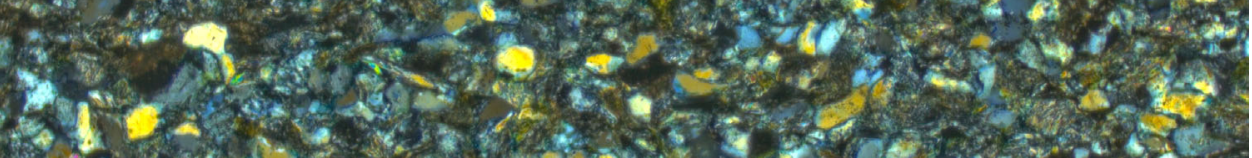

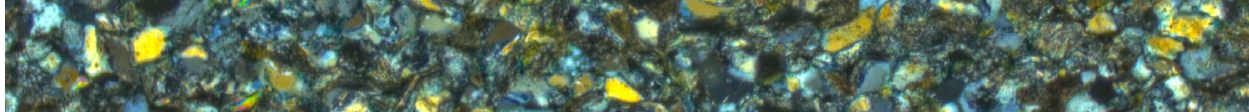

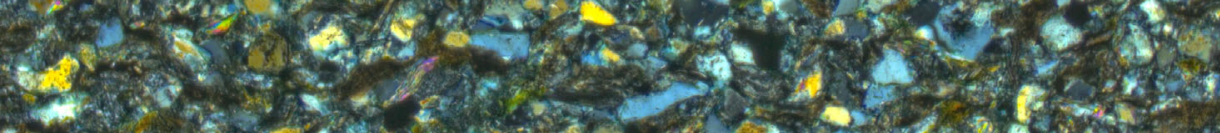

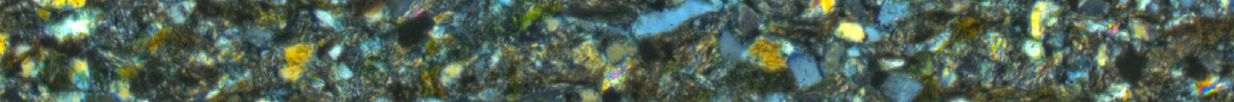

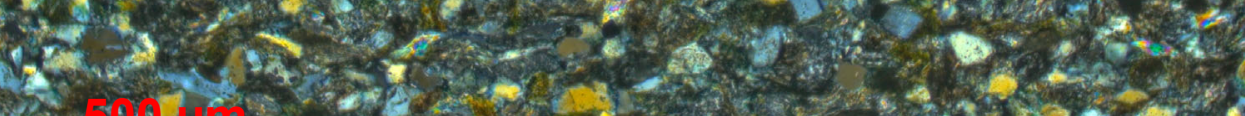

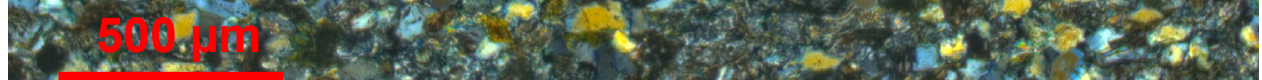

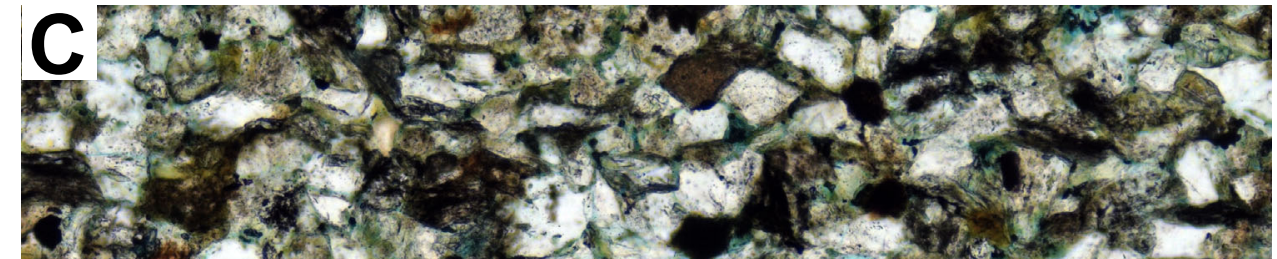

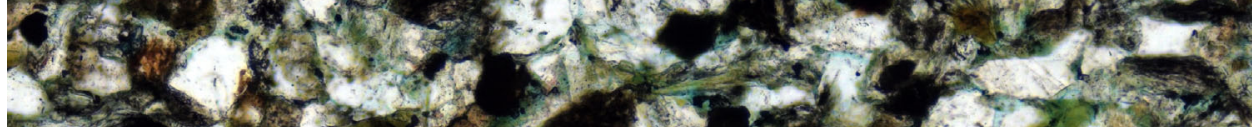

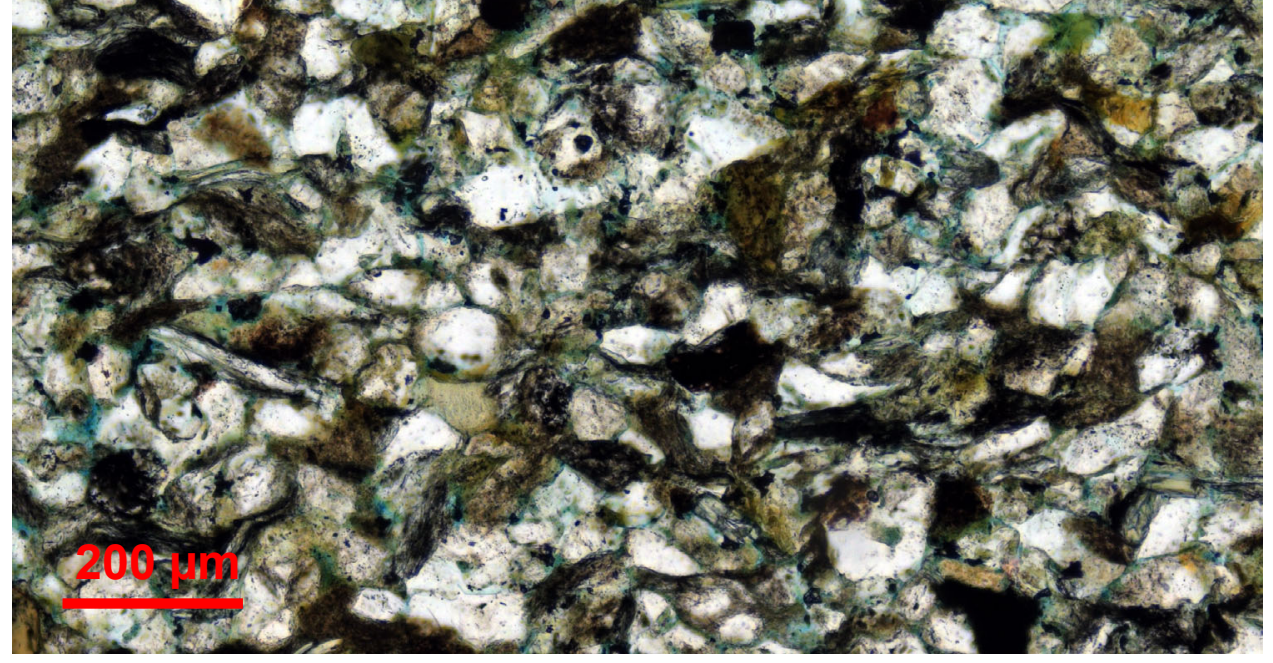

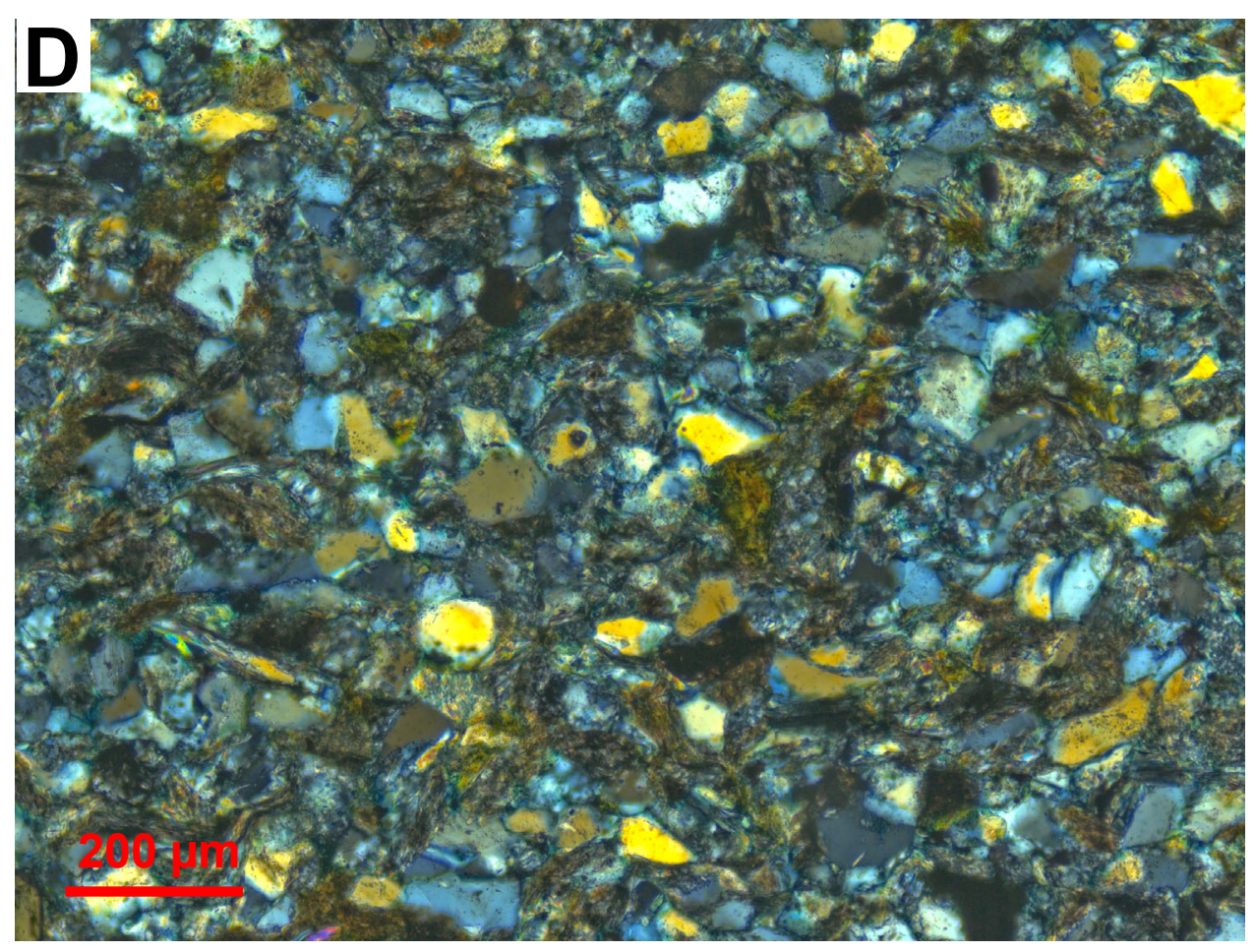


Fig 060 - 00DL022-221.0

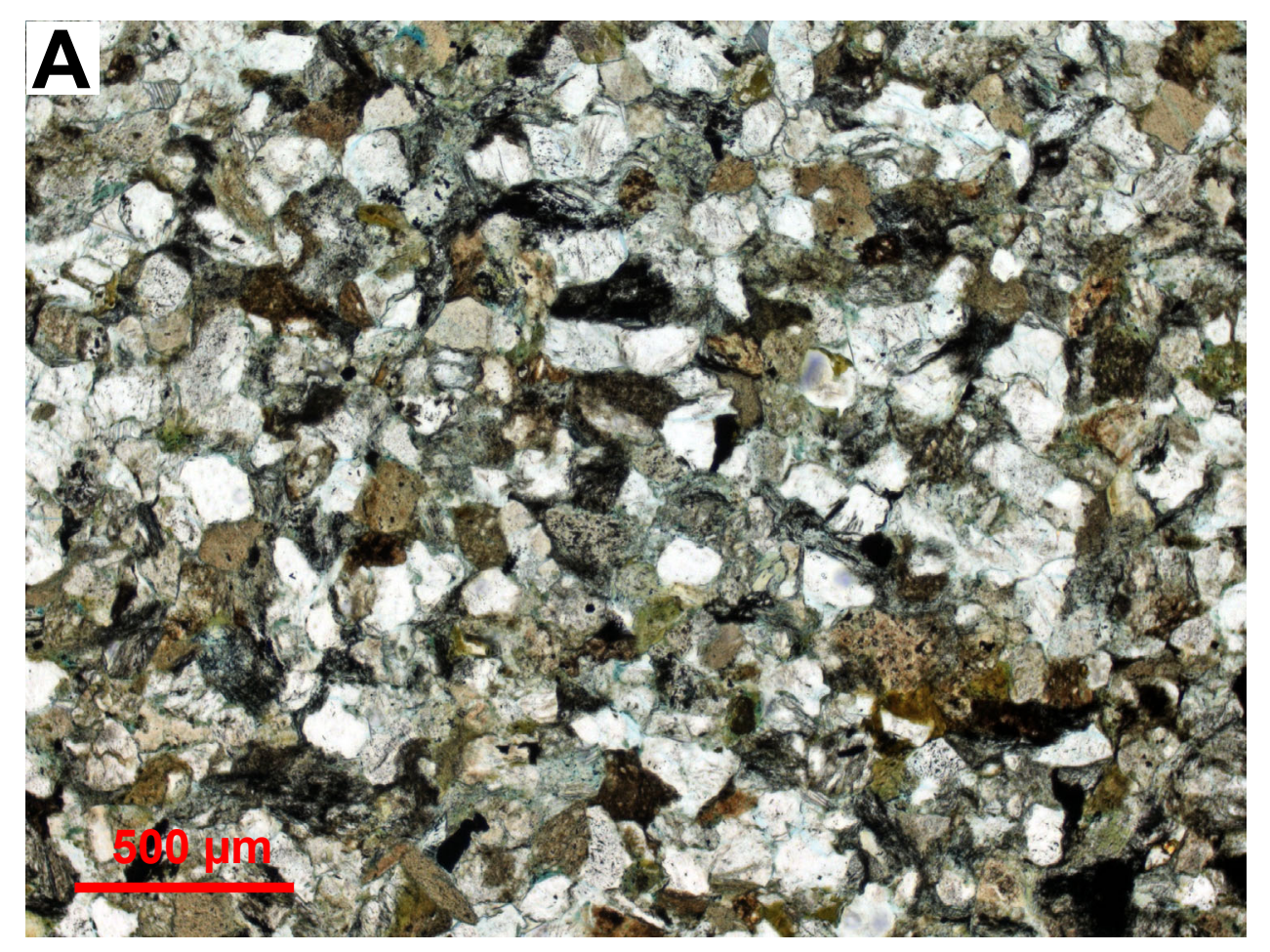

$B$ B

- 0102235

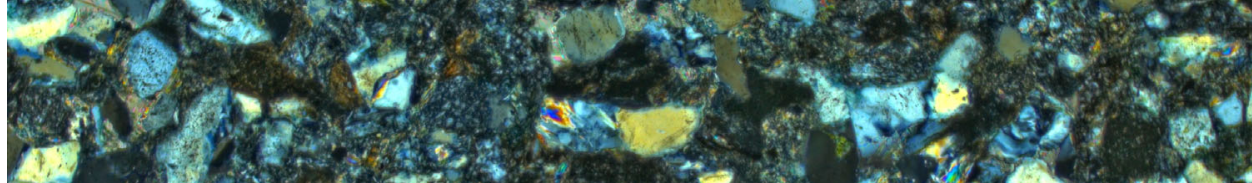
- $40-5,5=$

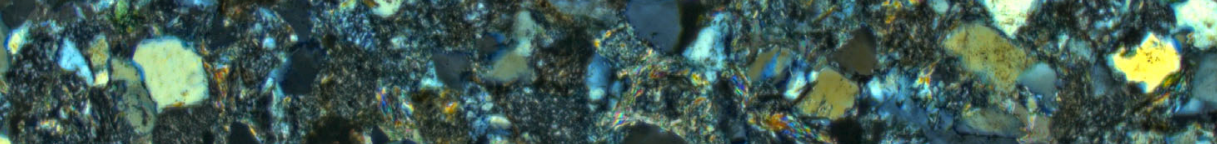
- wo $=2005$

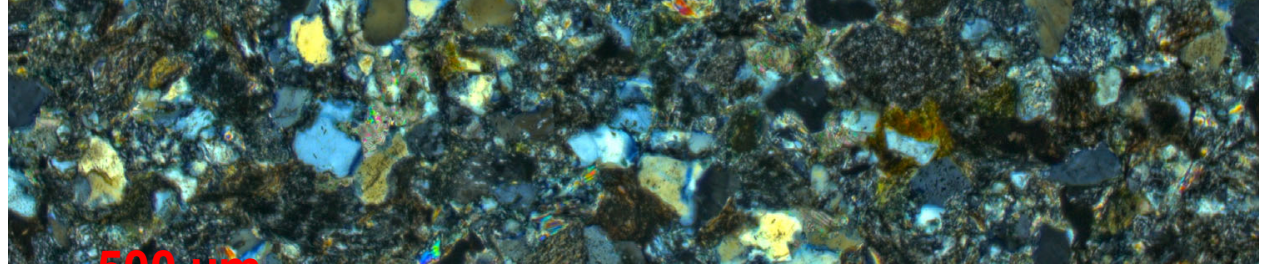

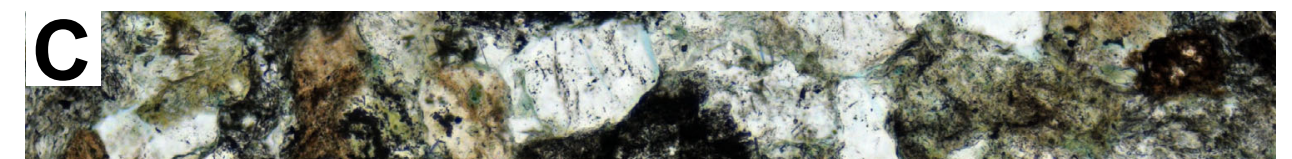
$(x-2)-25$

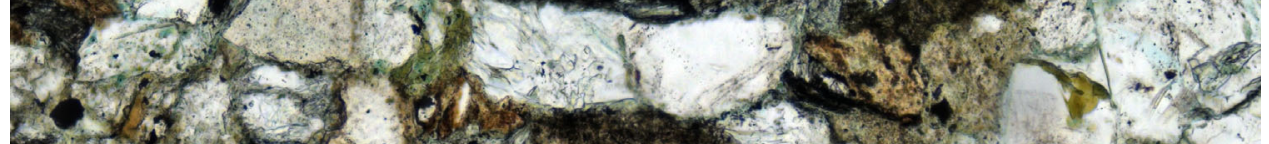
$y^{2}+y^{2}$
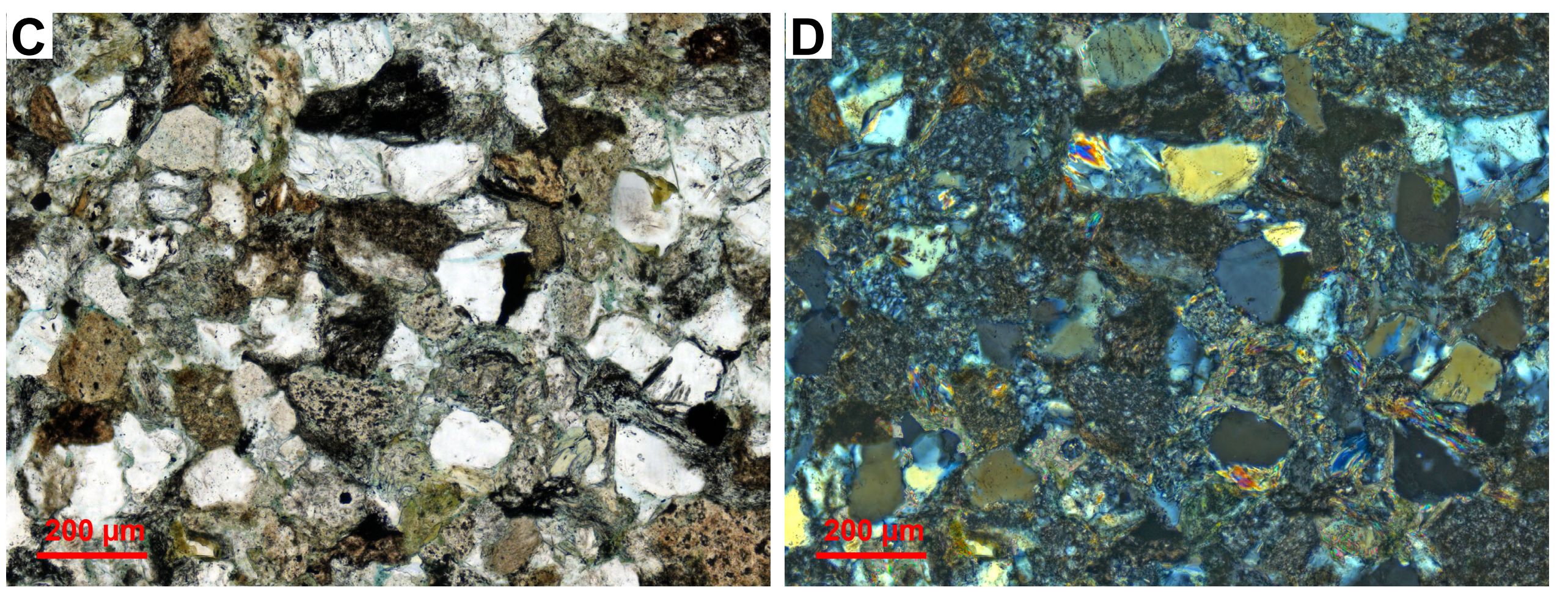
Fig 061 - 00DL022-224.8
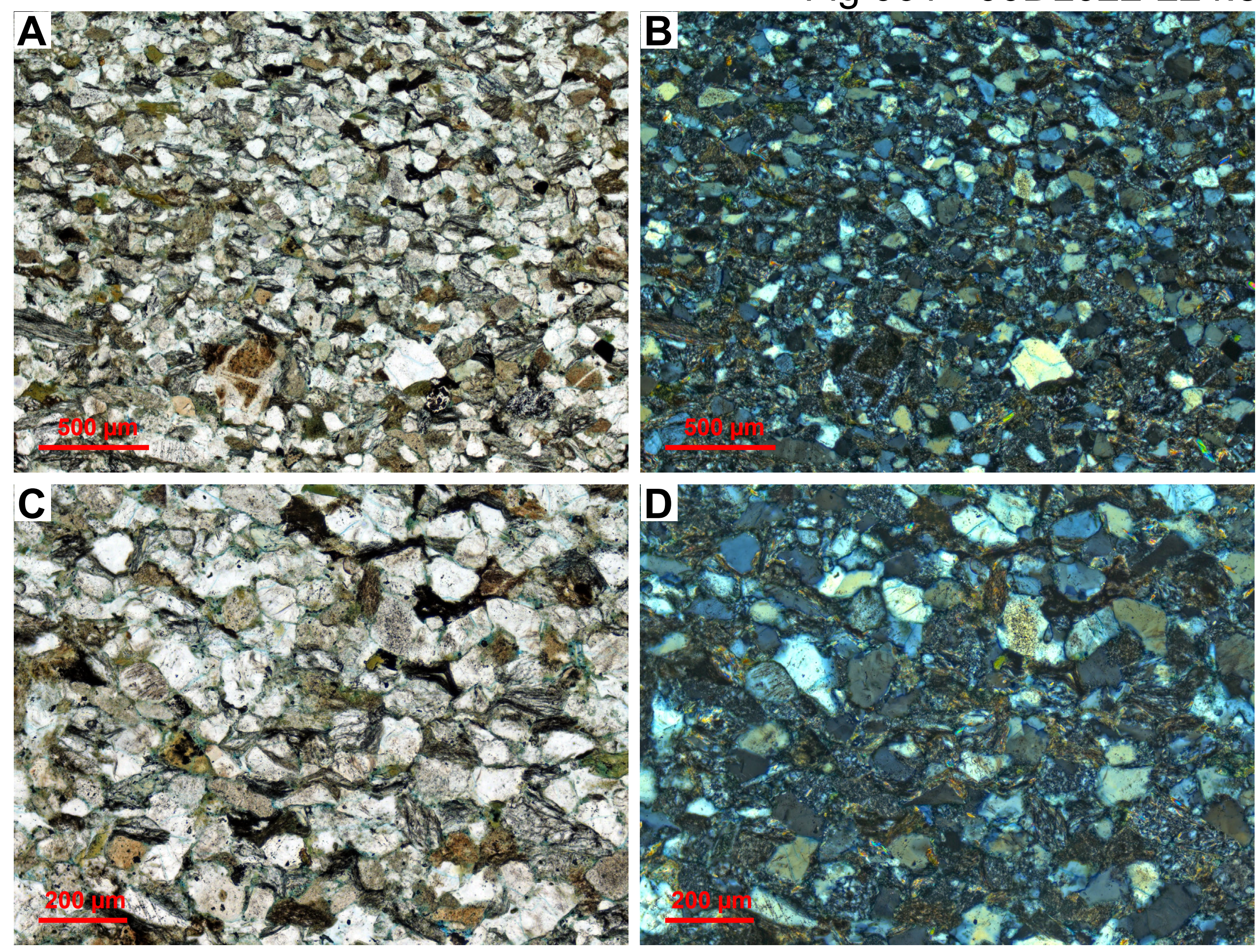
Fig 062 - 00DL022-232.0
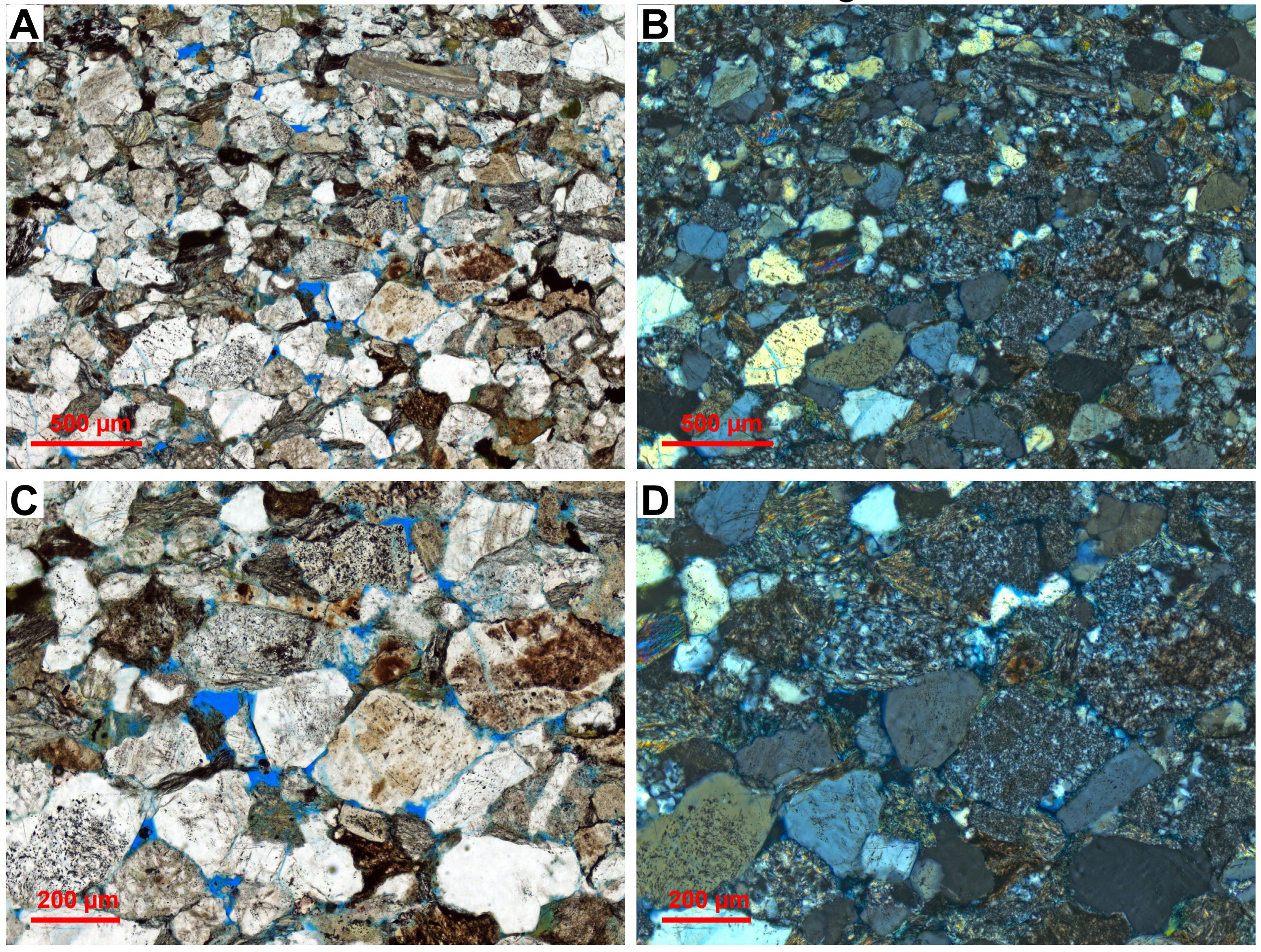
Fig 063 - 00DL022-235.0
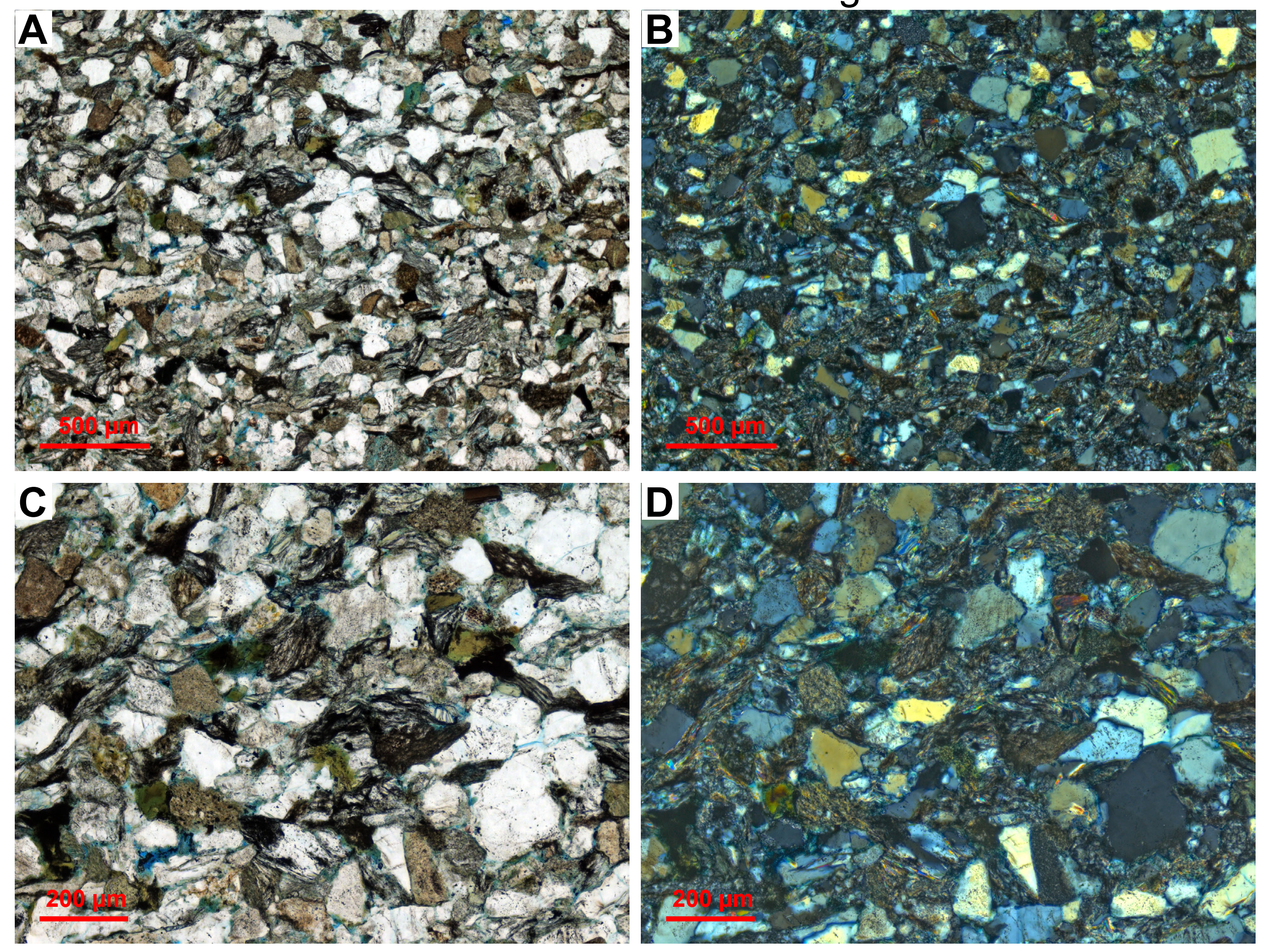
Fig 064 - 00DL022-237.6

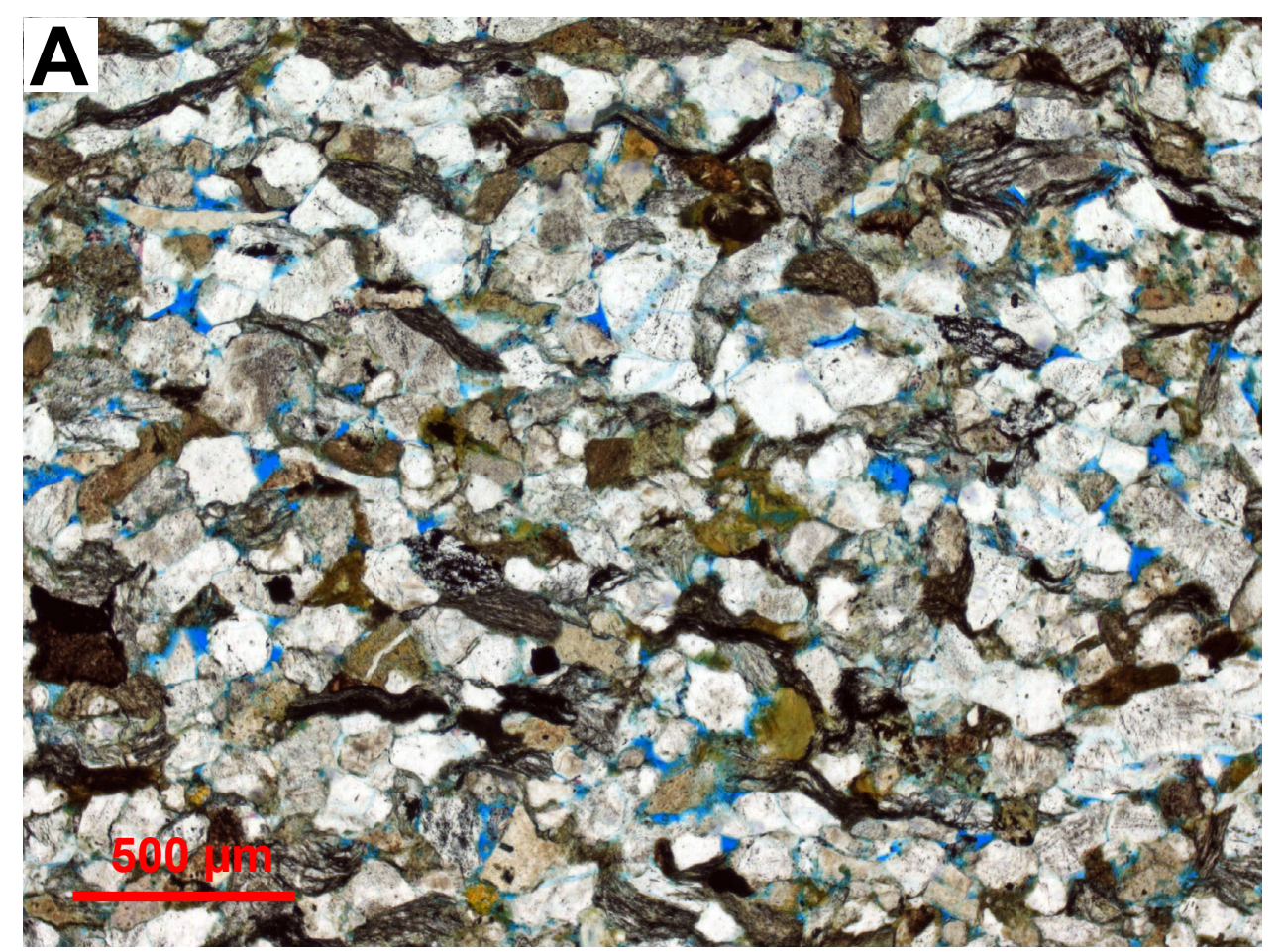

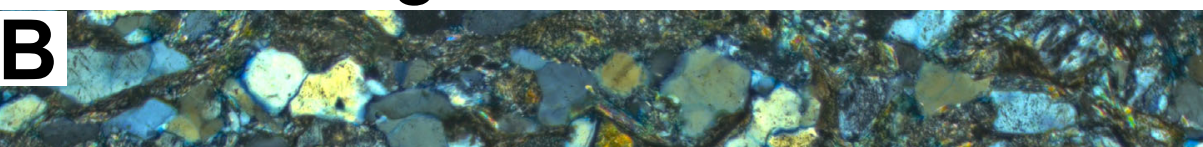

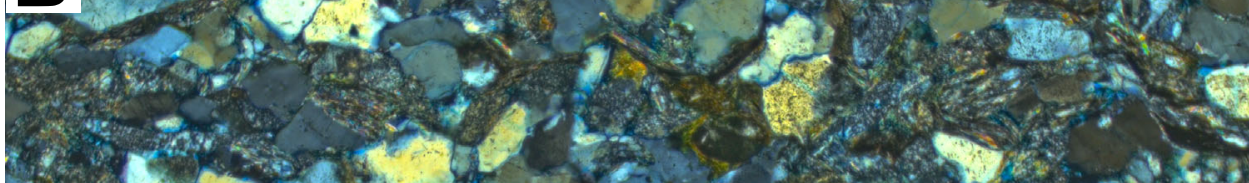

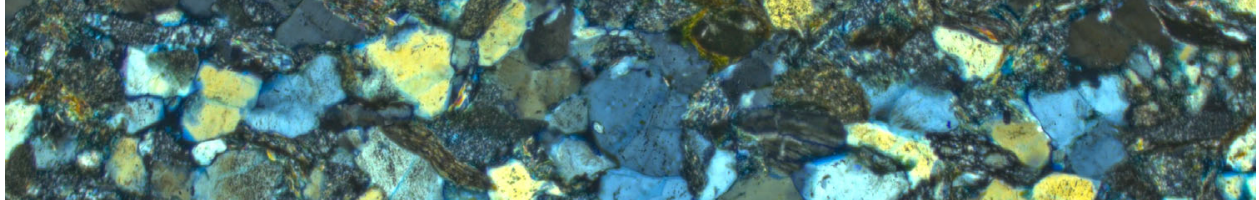

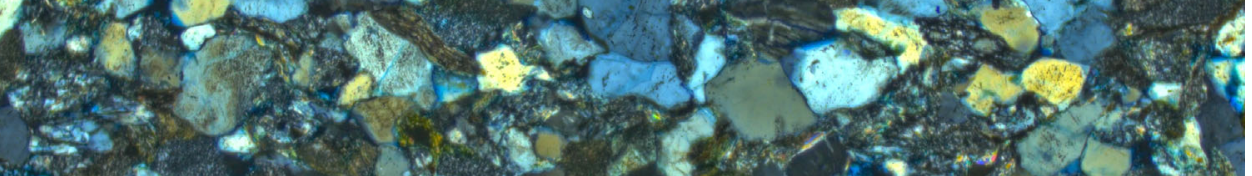

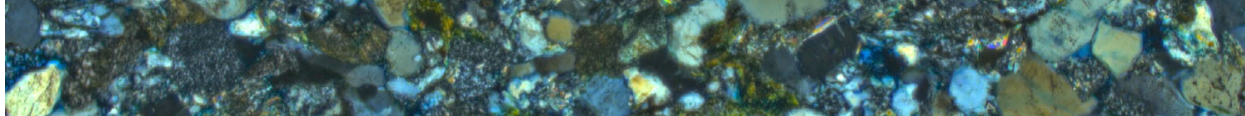

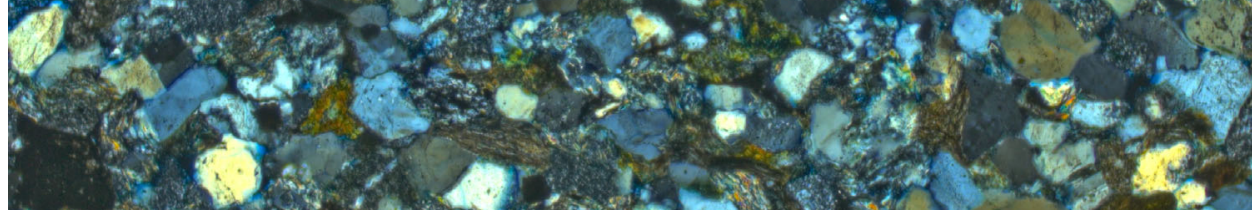
$16,5 \times 2,27$, 5 - . 2.

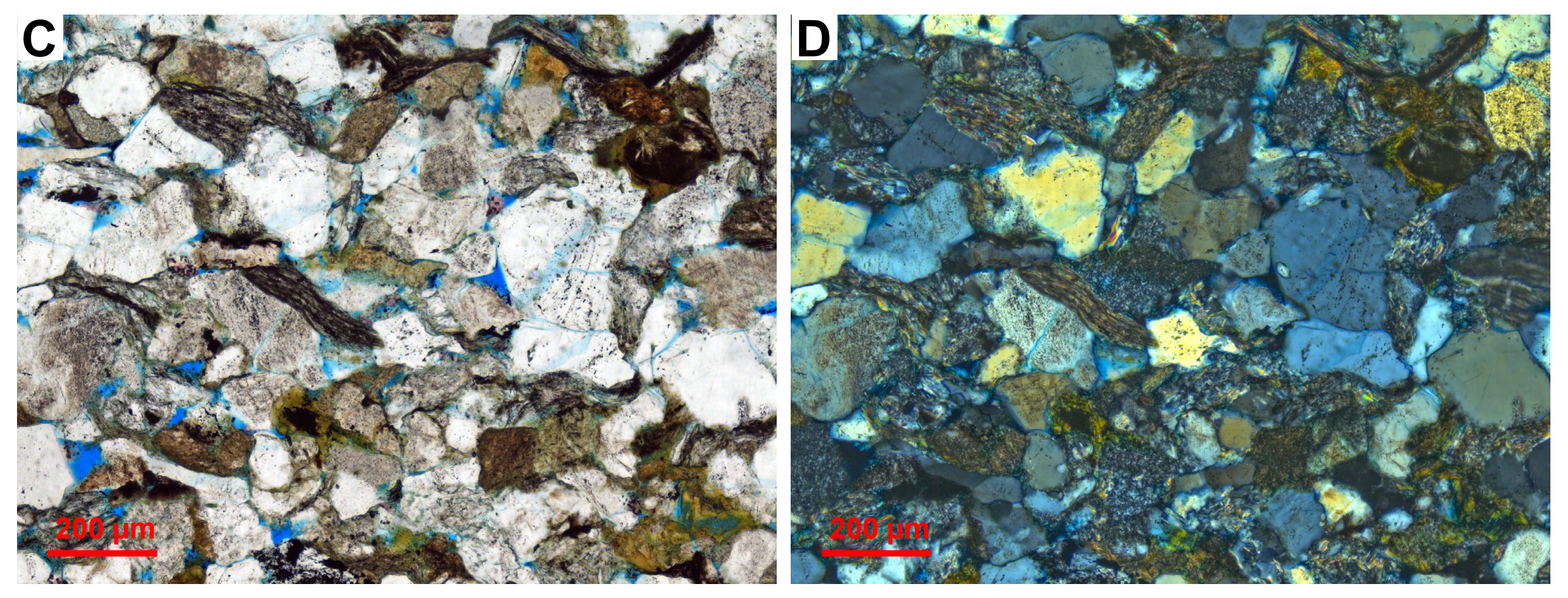


Fig 065 - 00DL022-241.4

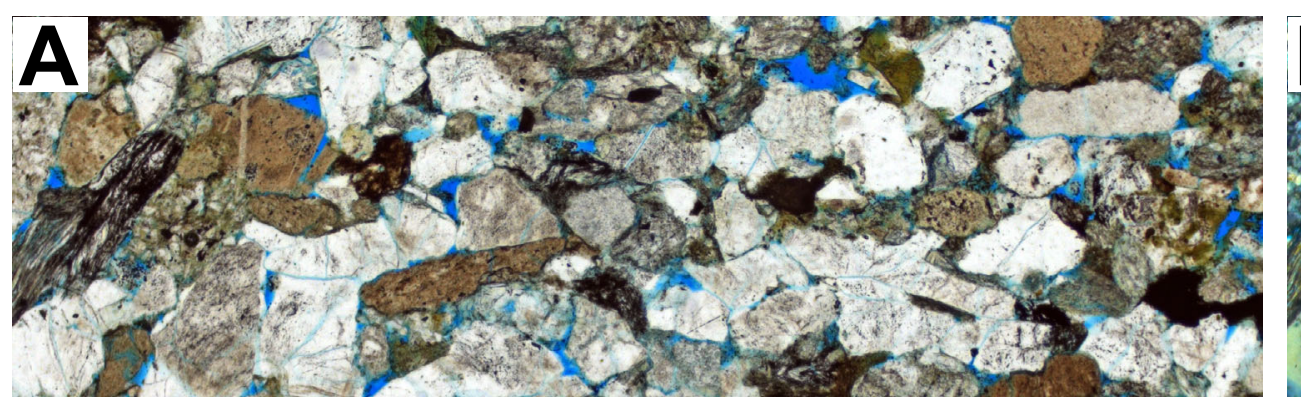
6.7.2. 205

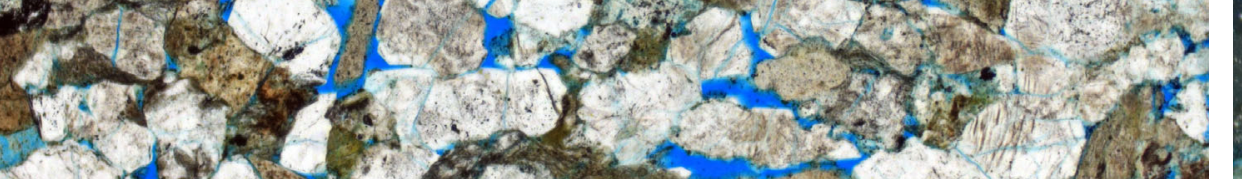
1) $50-1$

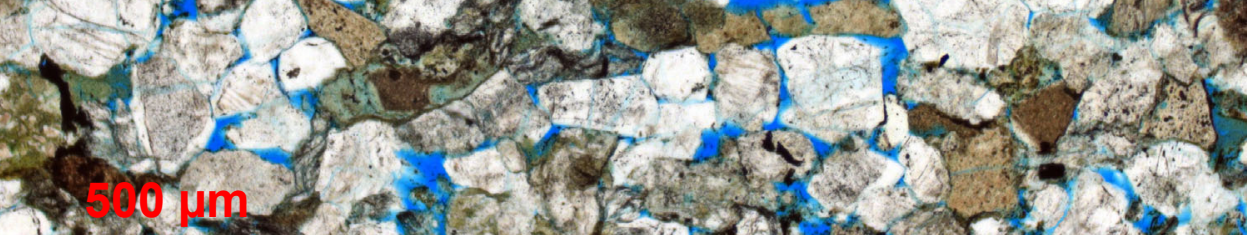
yor chat ares

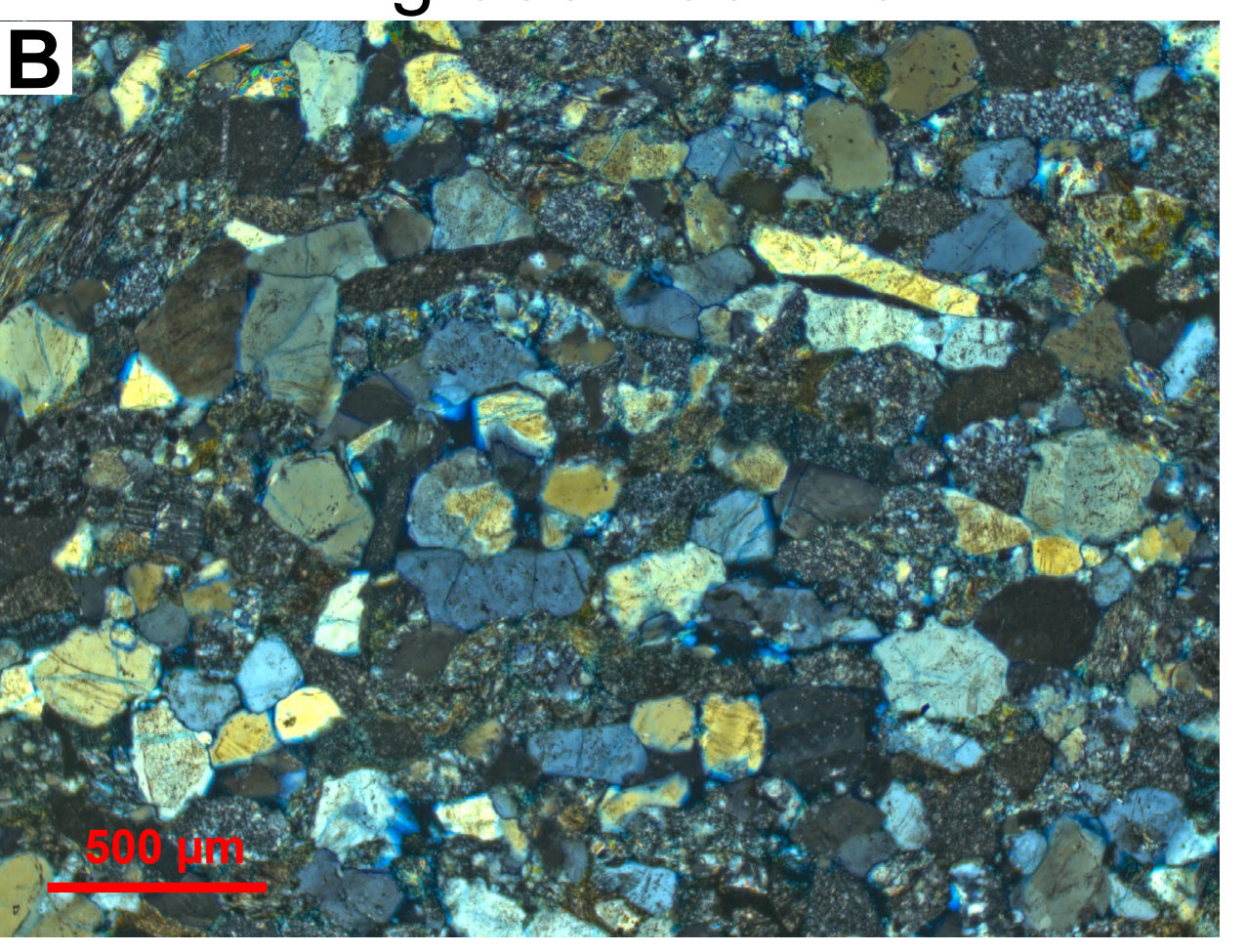

c ${ }^{2}+1$
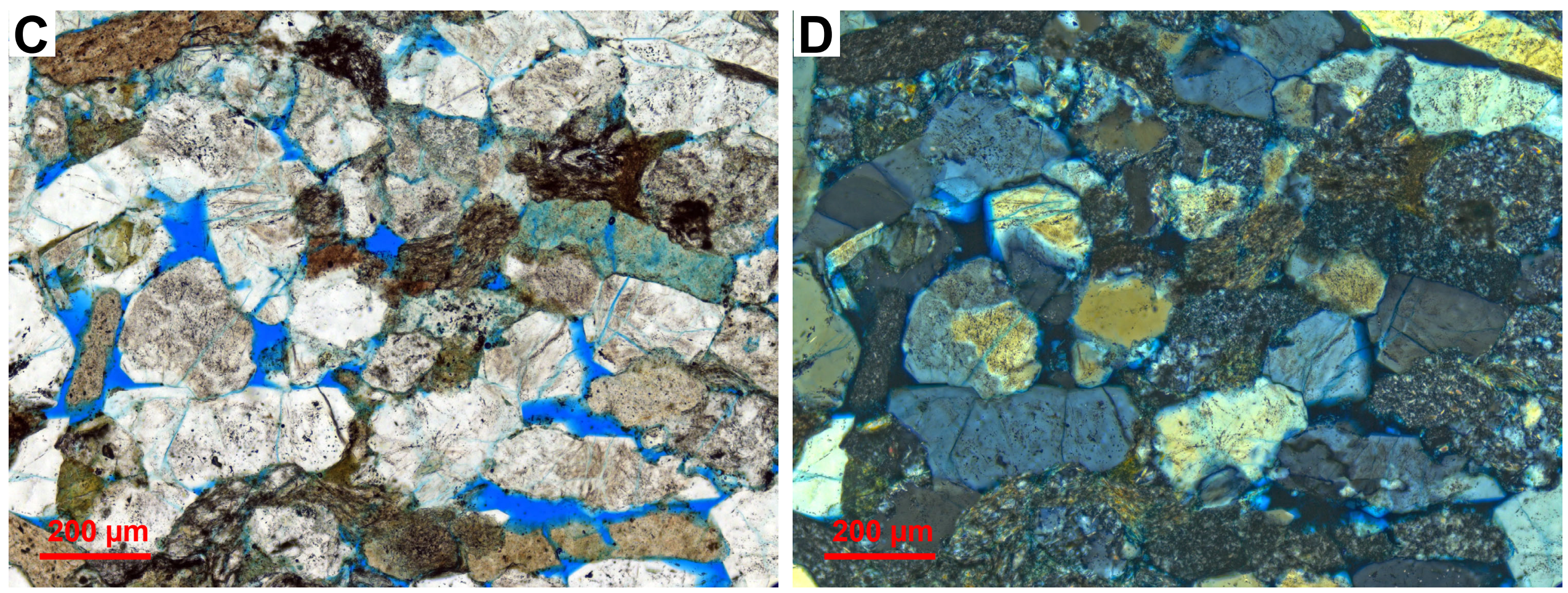
Fig 066 - 00DL022-244.0

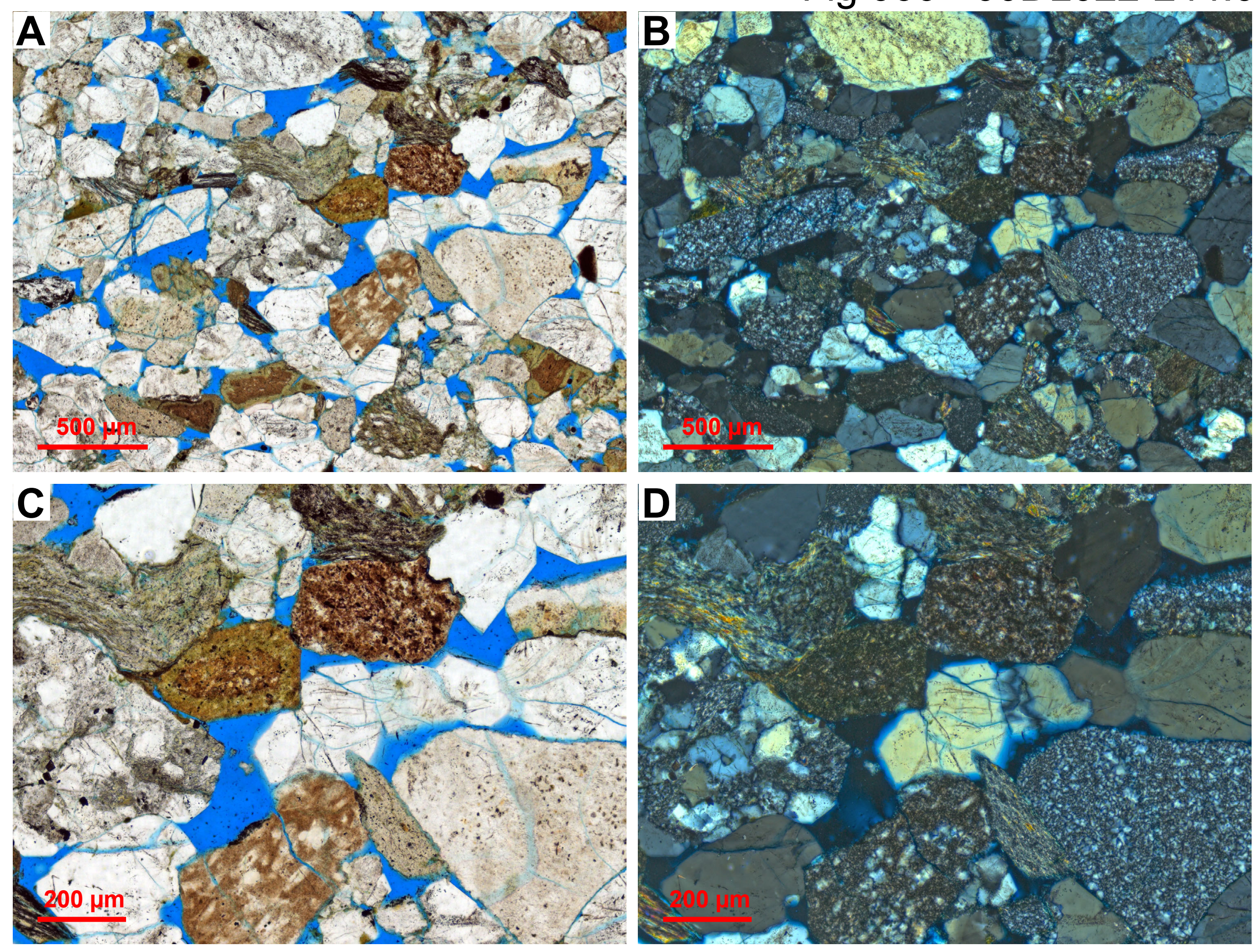


Fig 067 - 00DL022-244.4
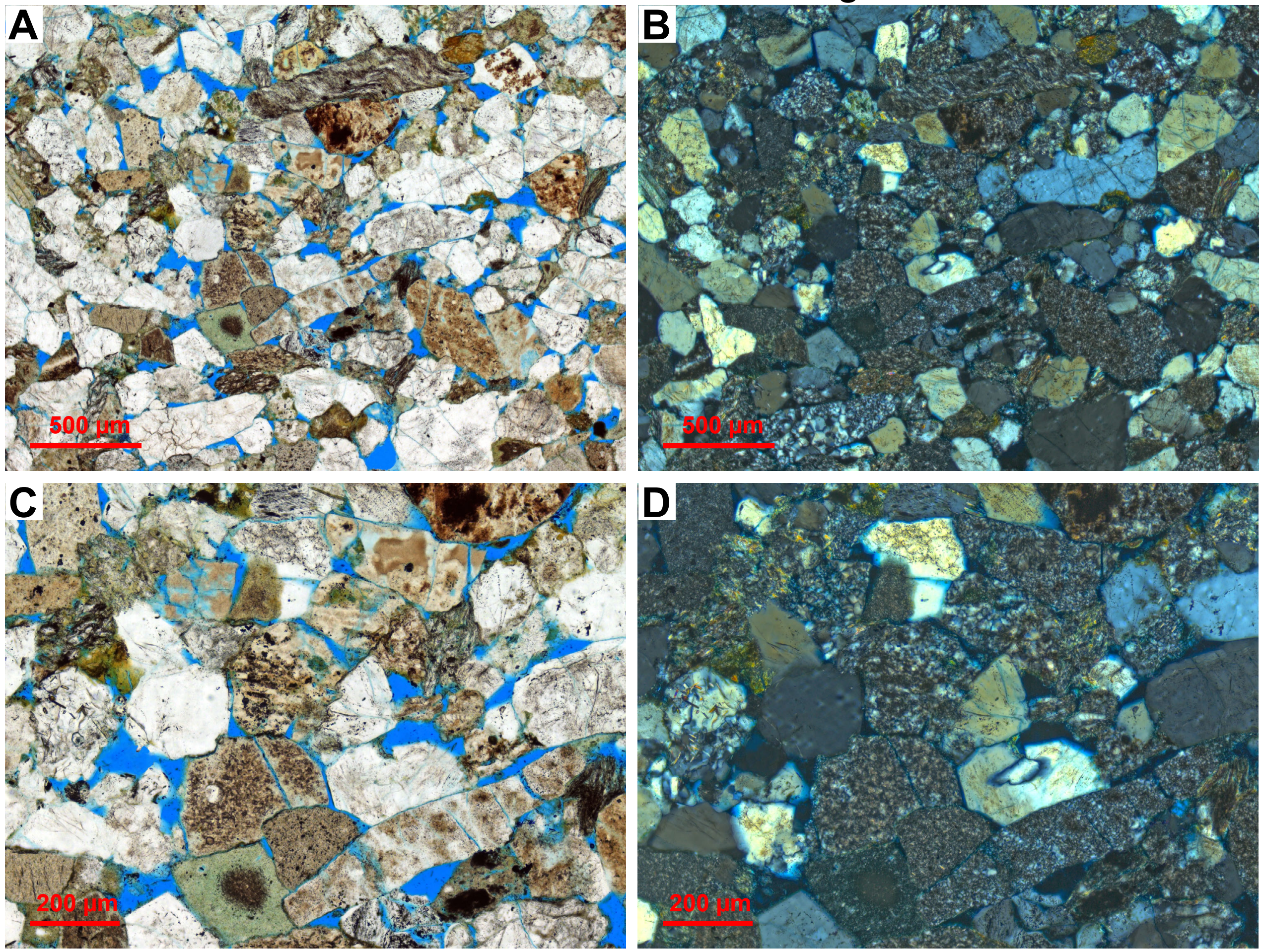
Fig 068 - 18PD003A
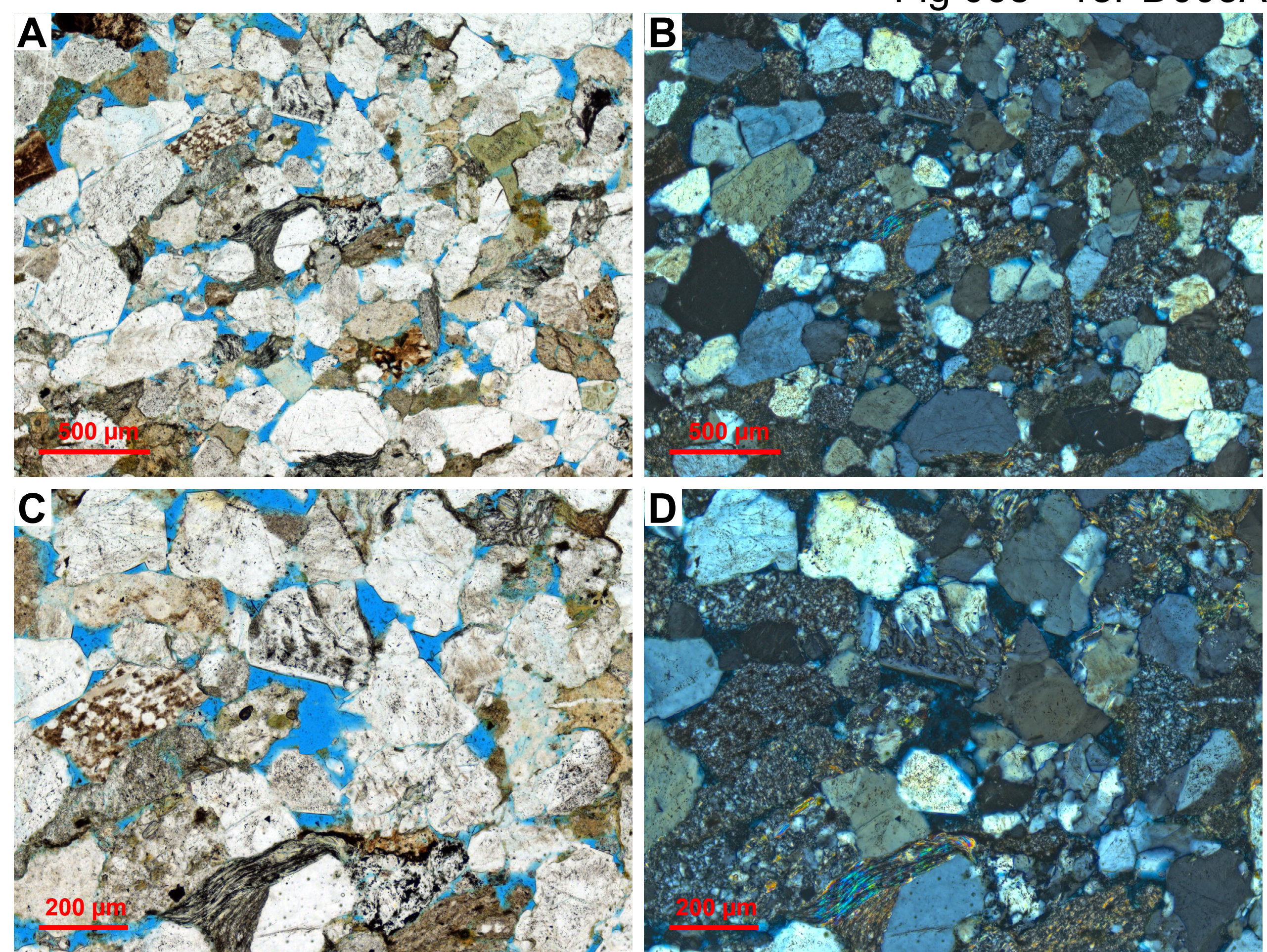

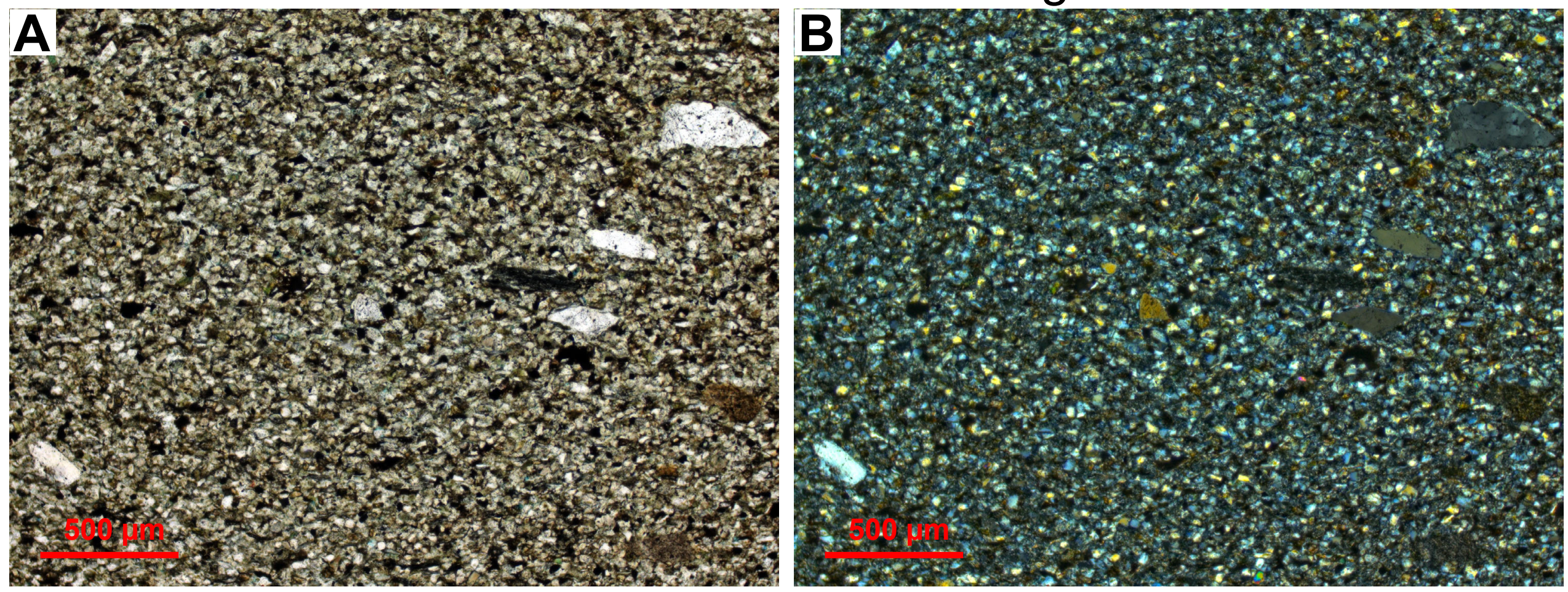

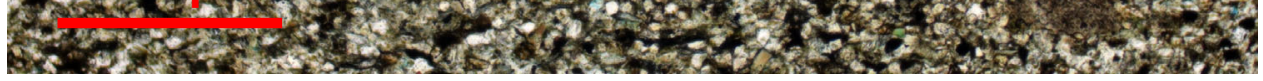

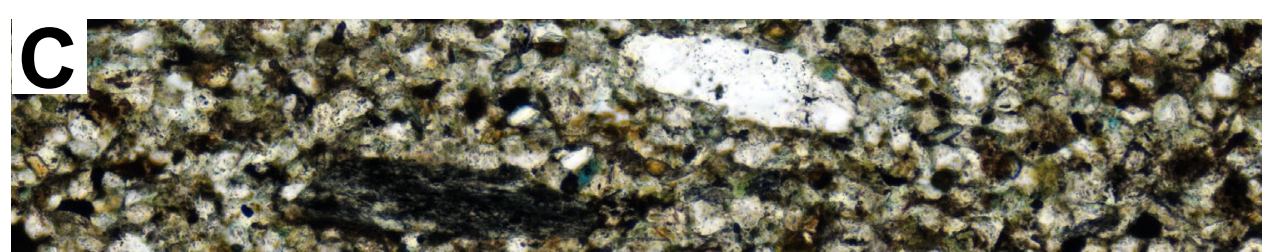

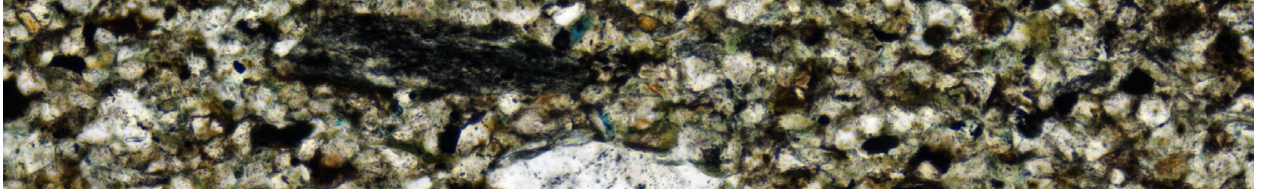

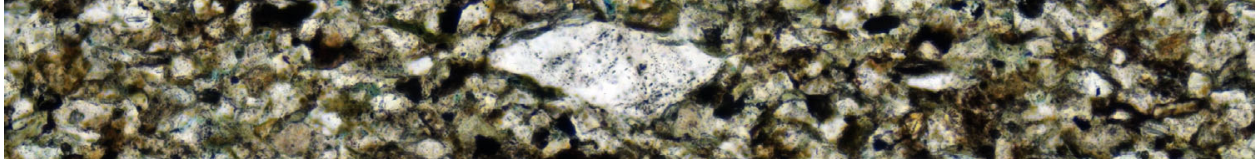

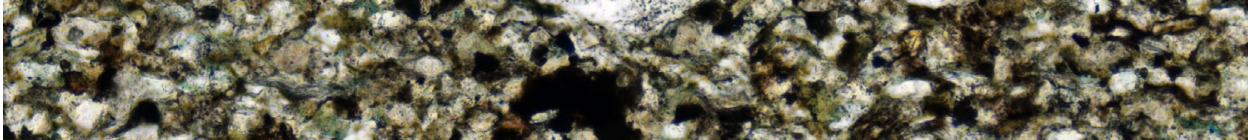

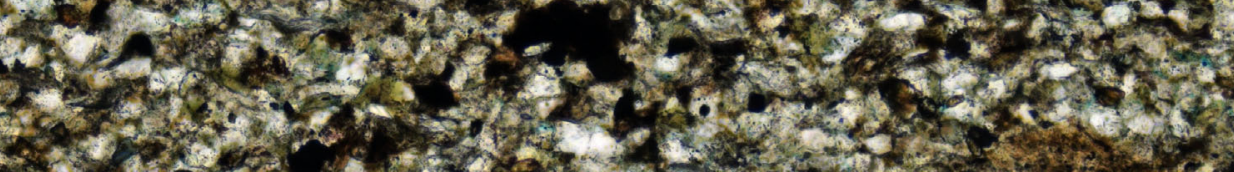

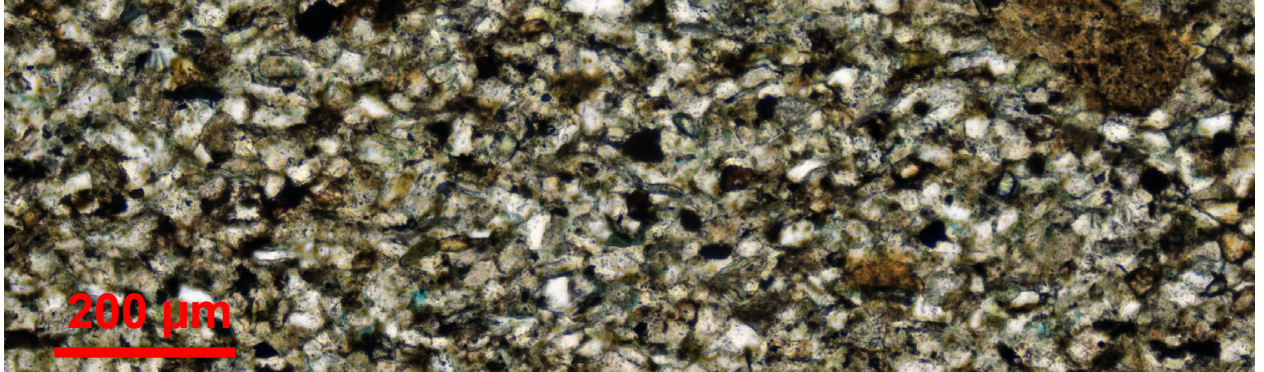

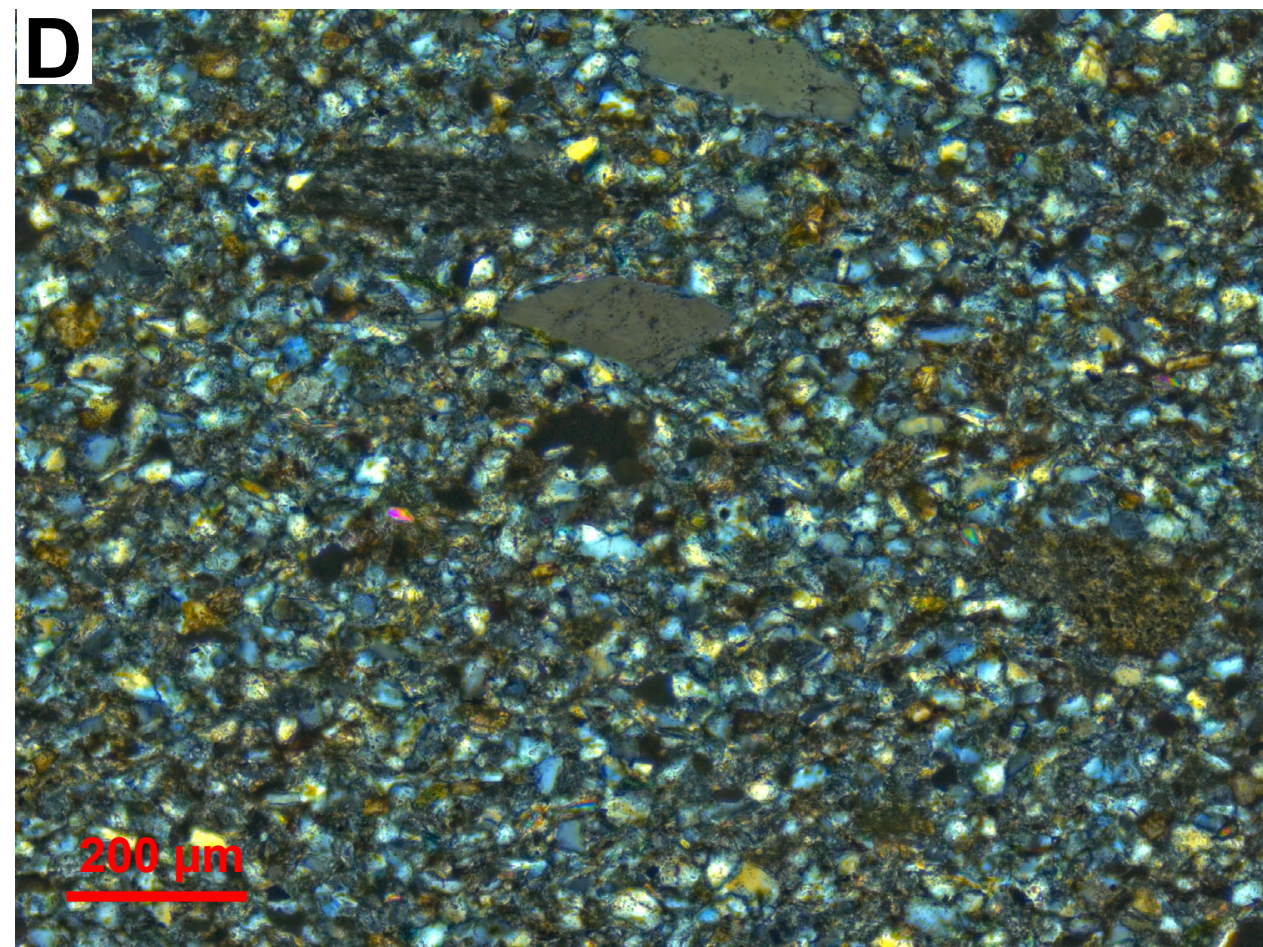


Fig 070 - 00DL017-024.35

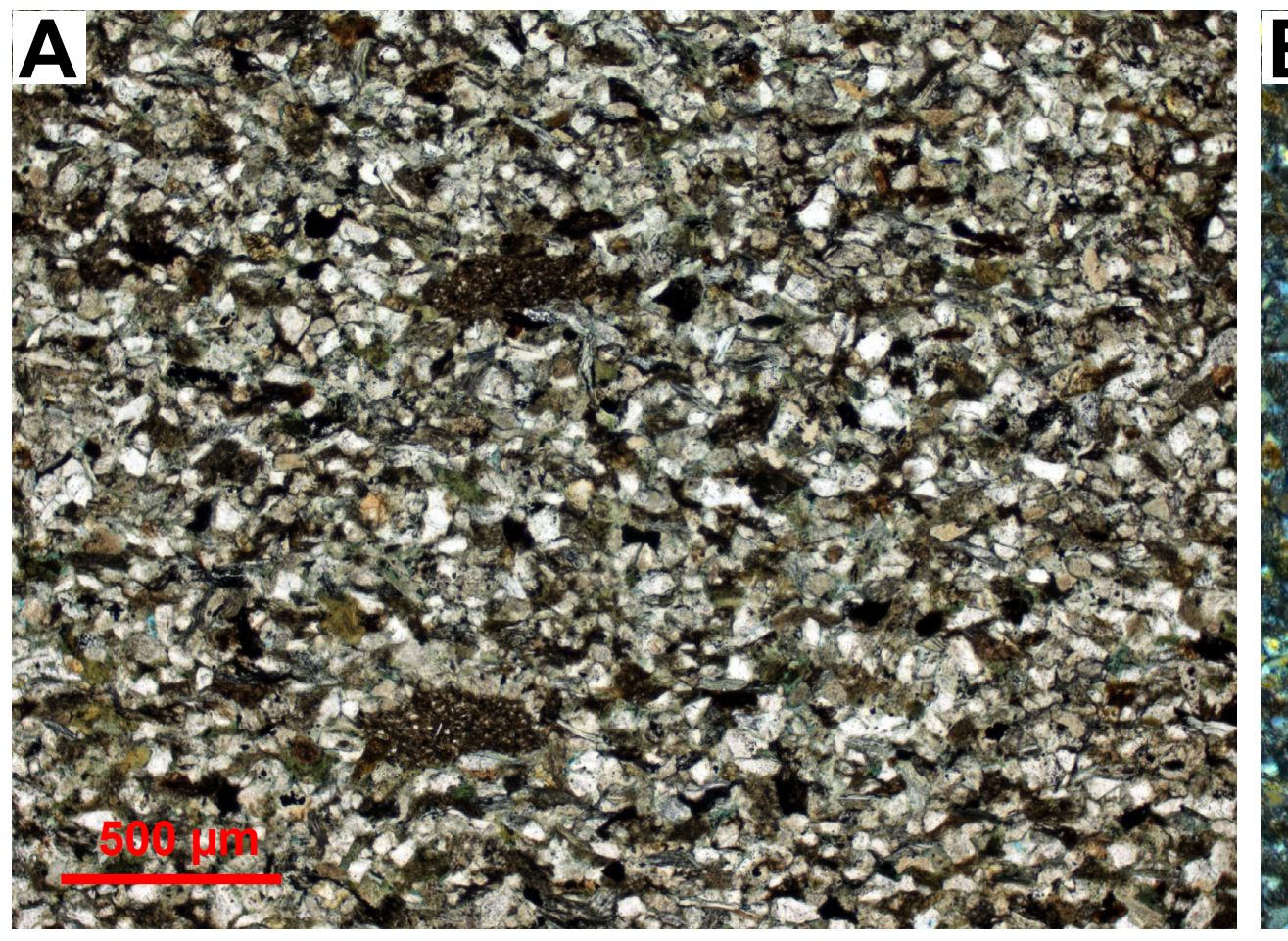

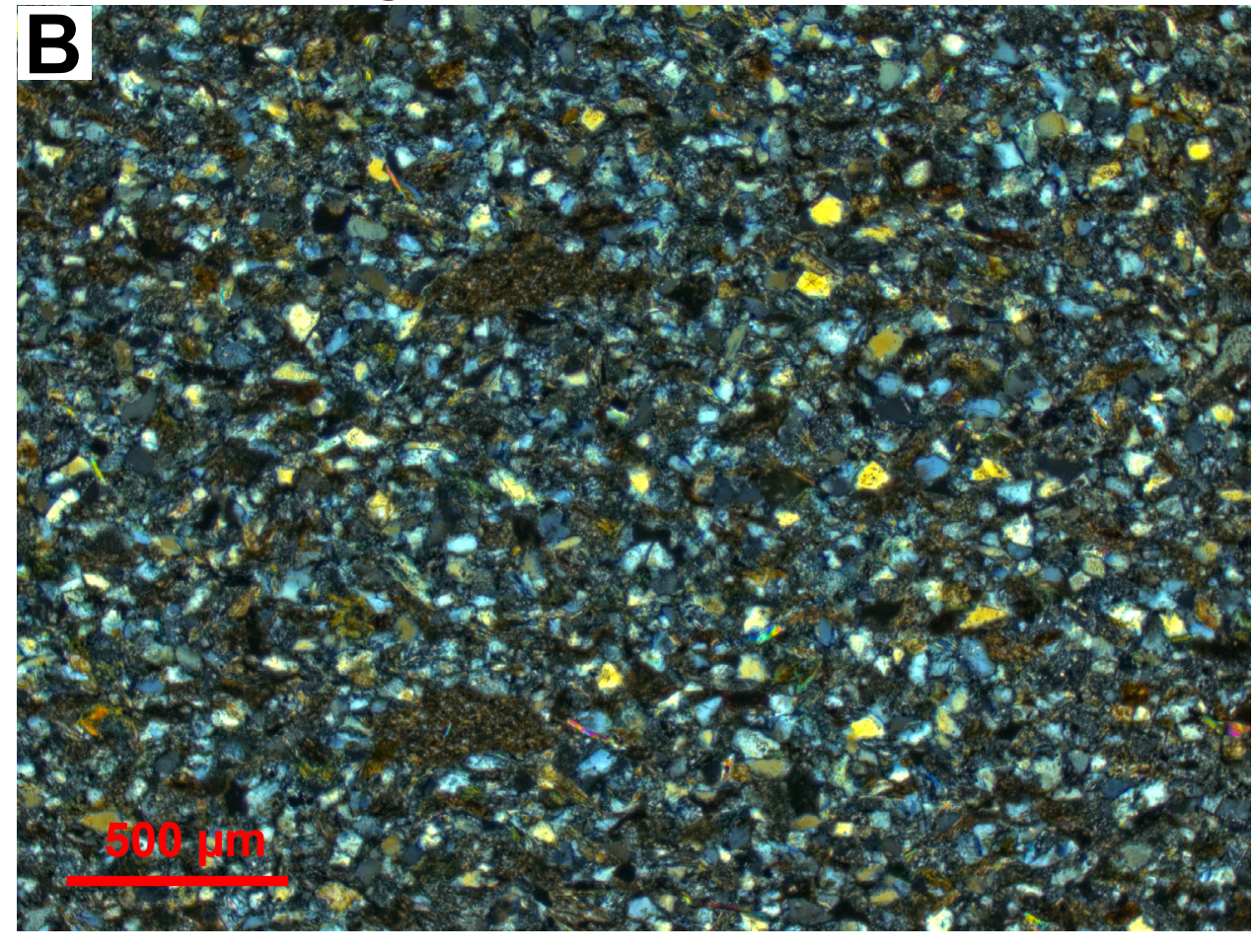

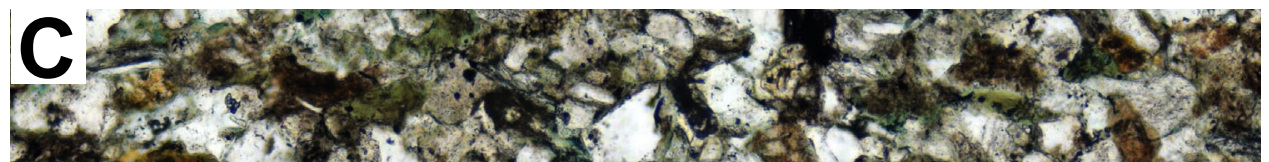
tan 10.

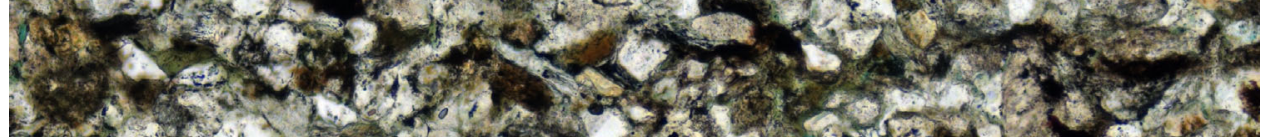

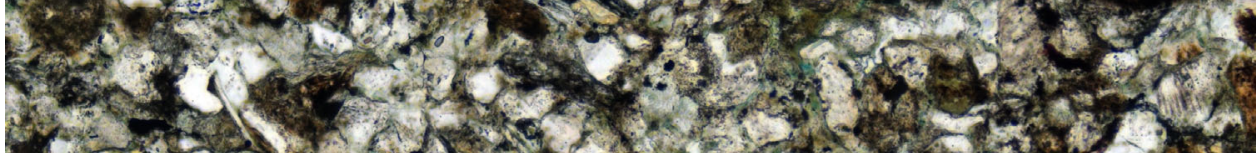
1. 2 7.470

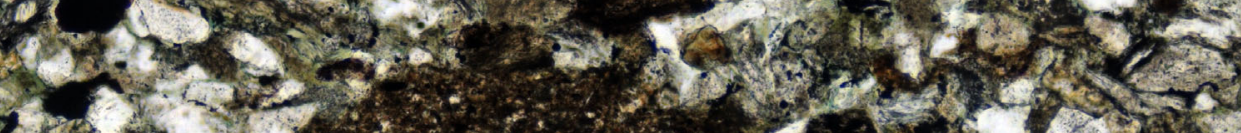
- r o 35,4

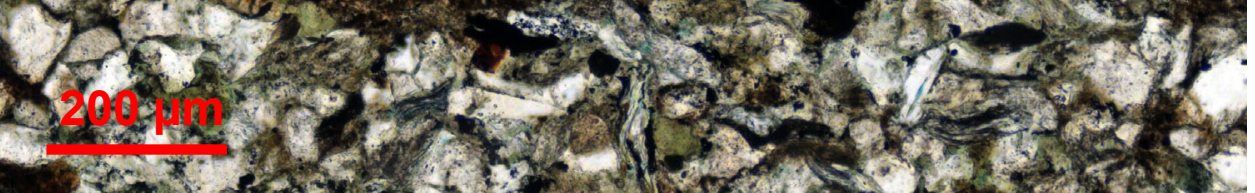

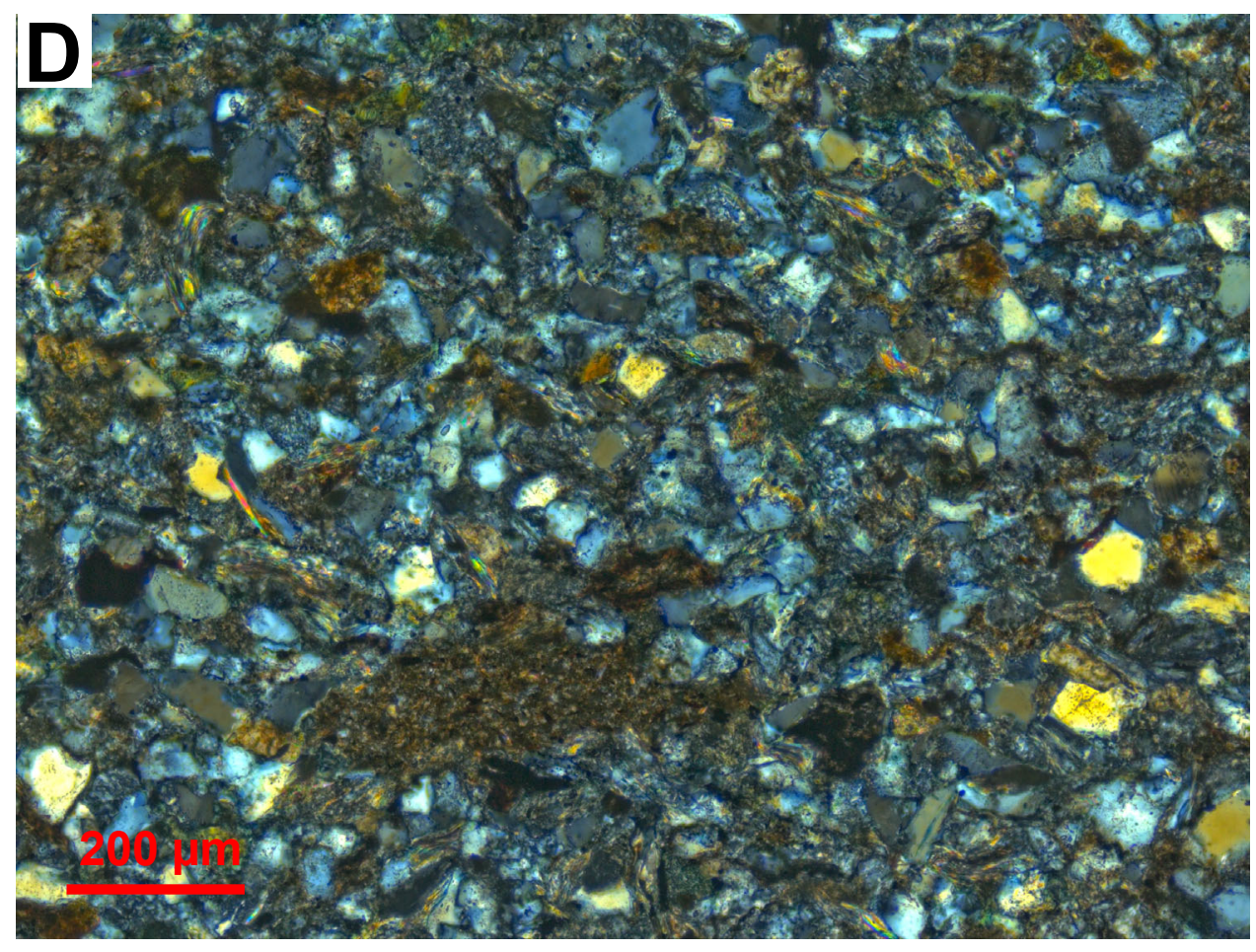




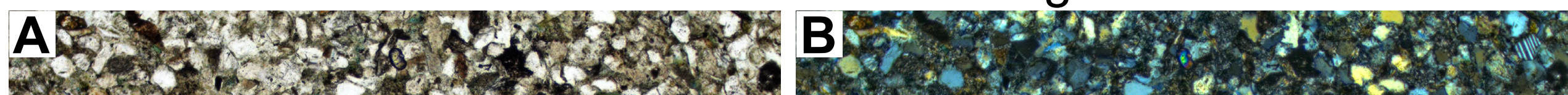

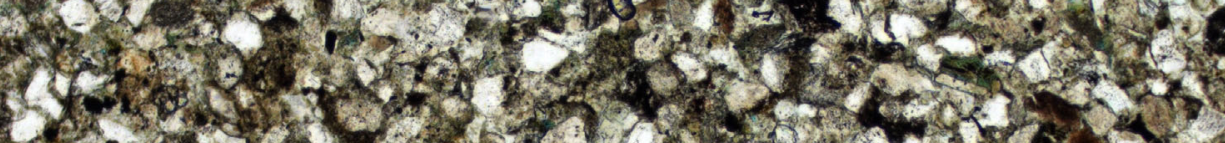

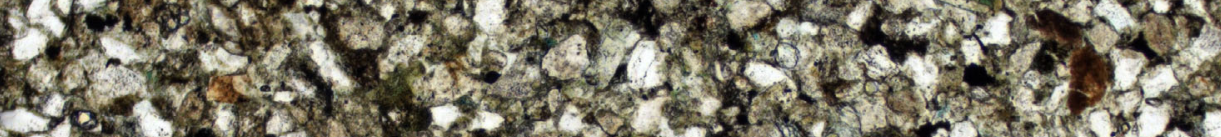

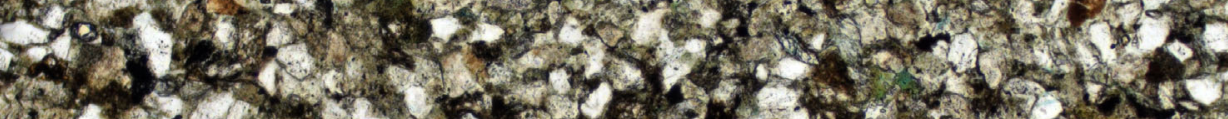

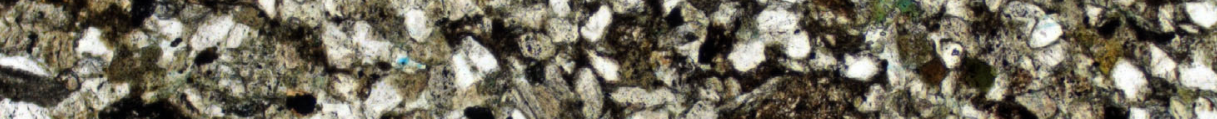

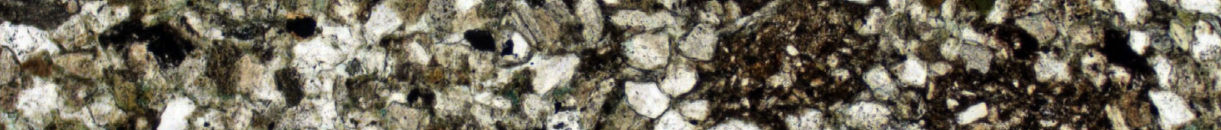

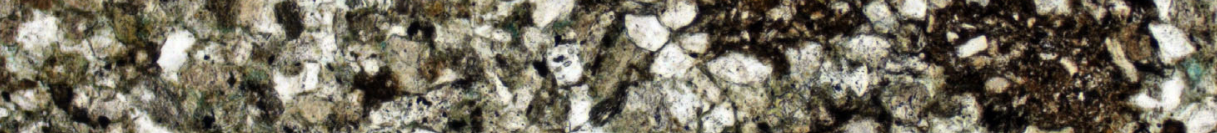

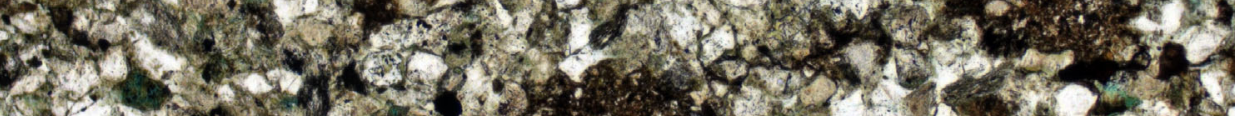

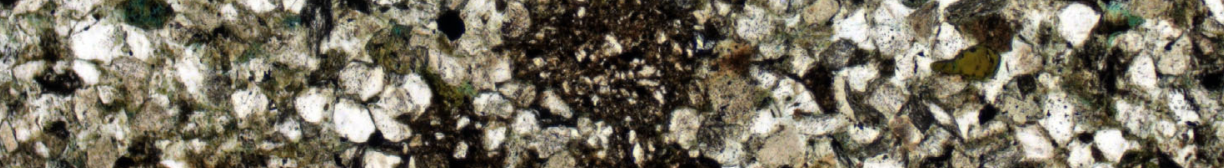

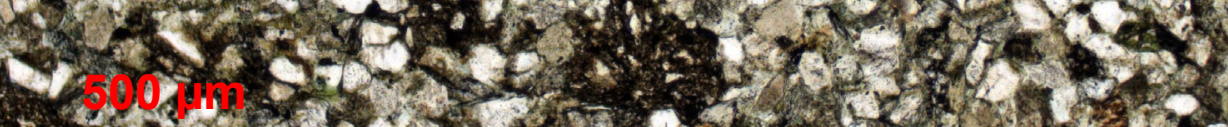

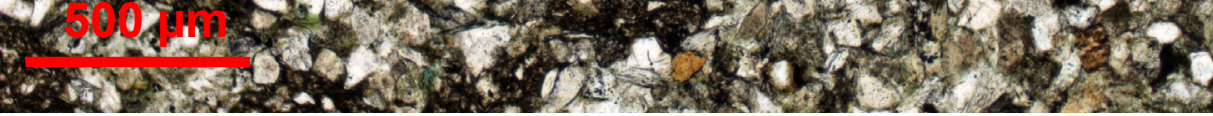

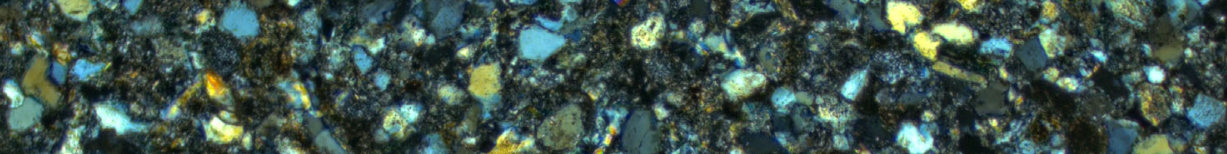

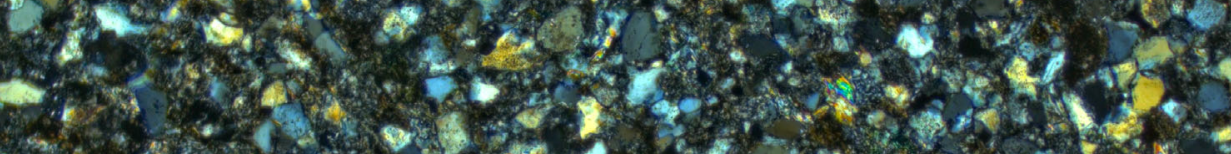

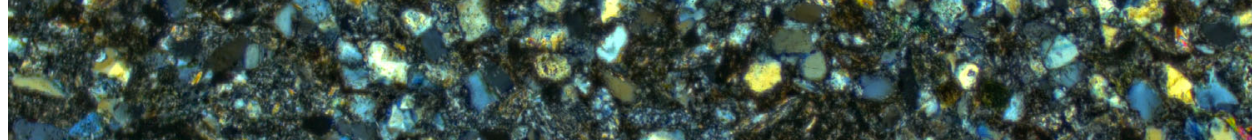

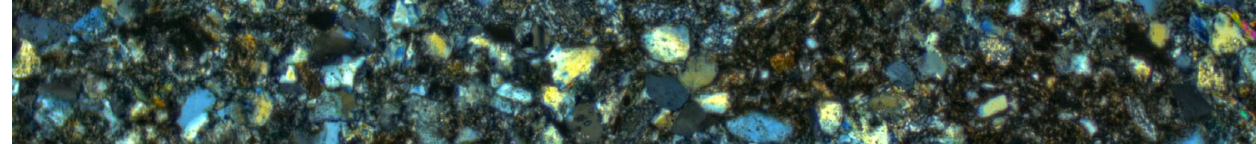

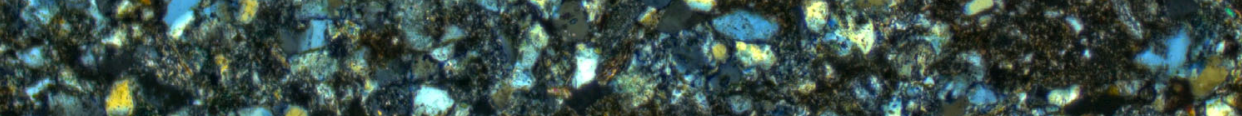

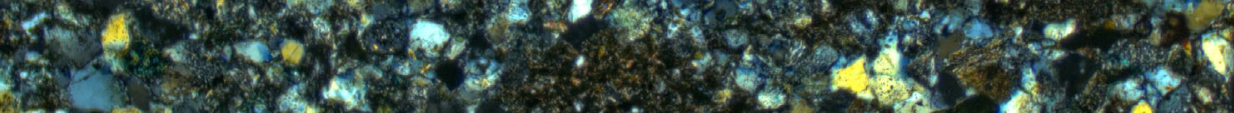

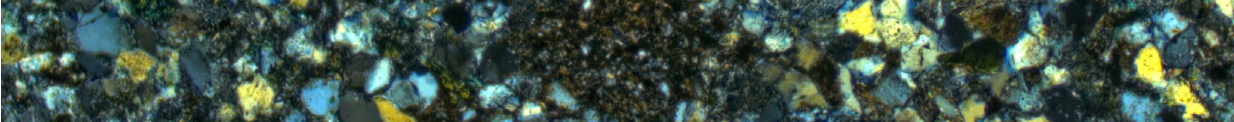

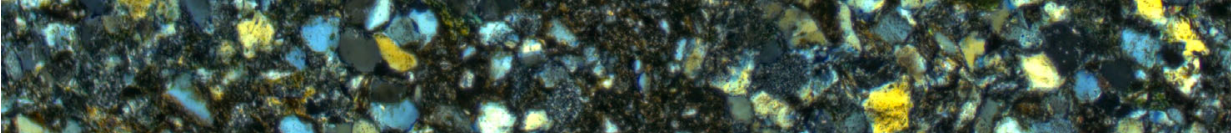

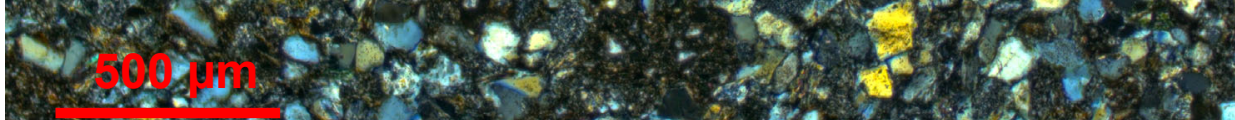
C.1.

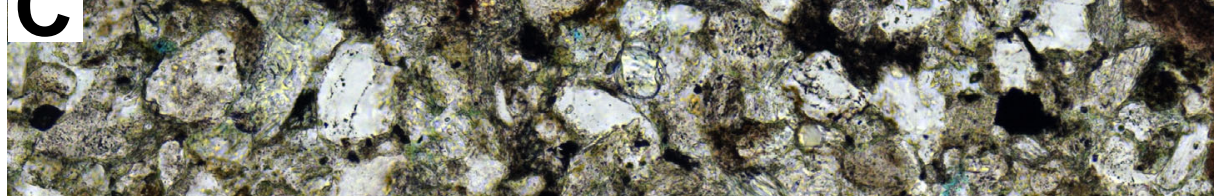

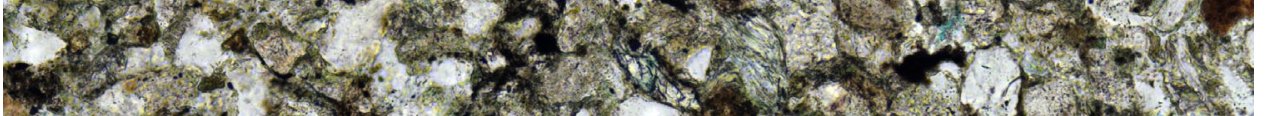

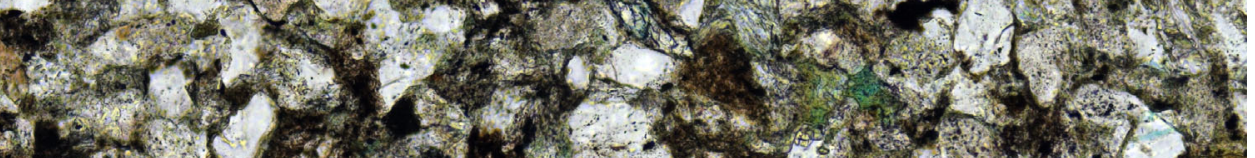

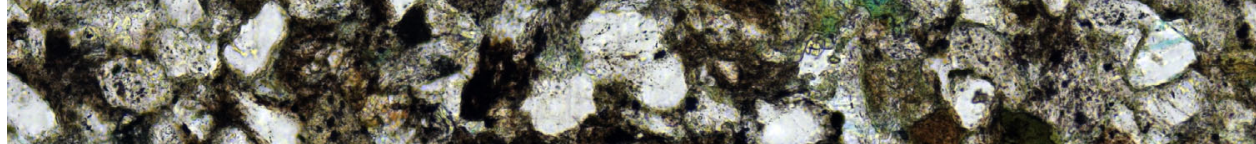

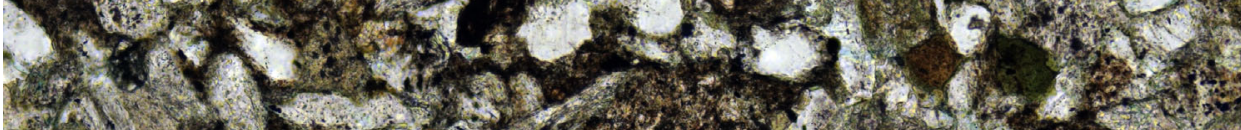

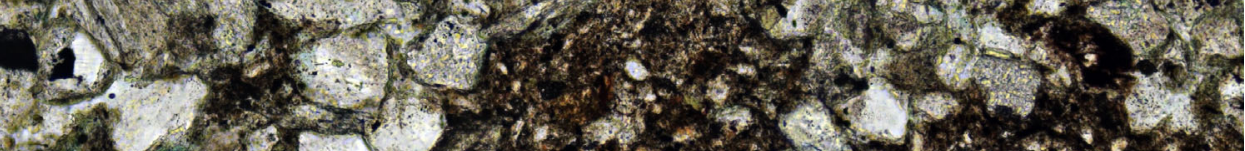

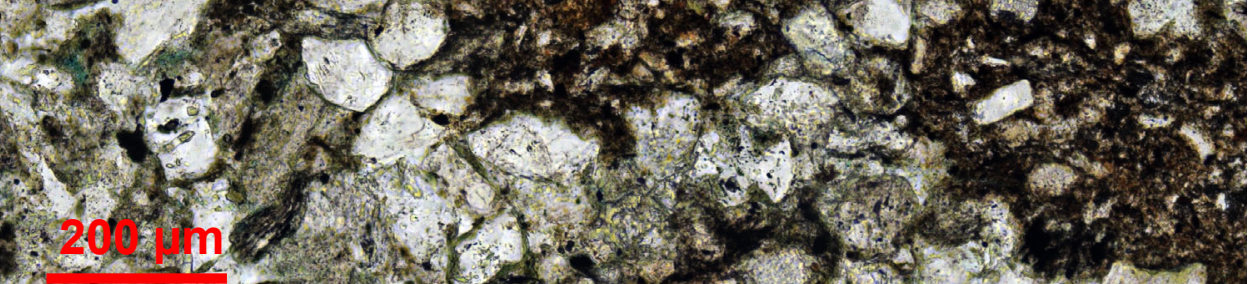

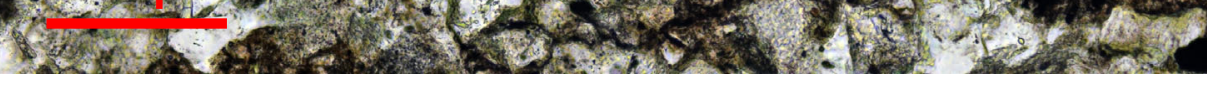

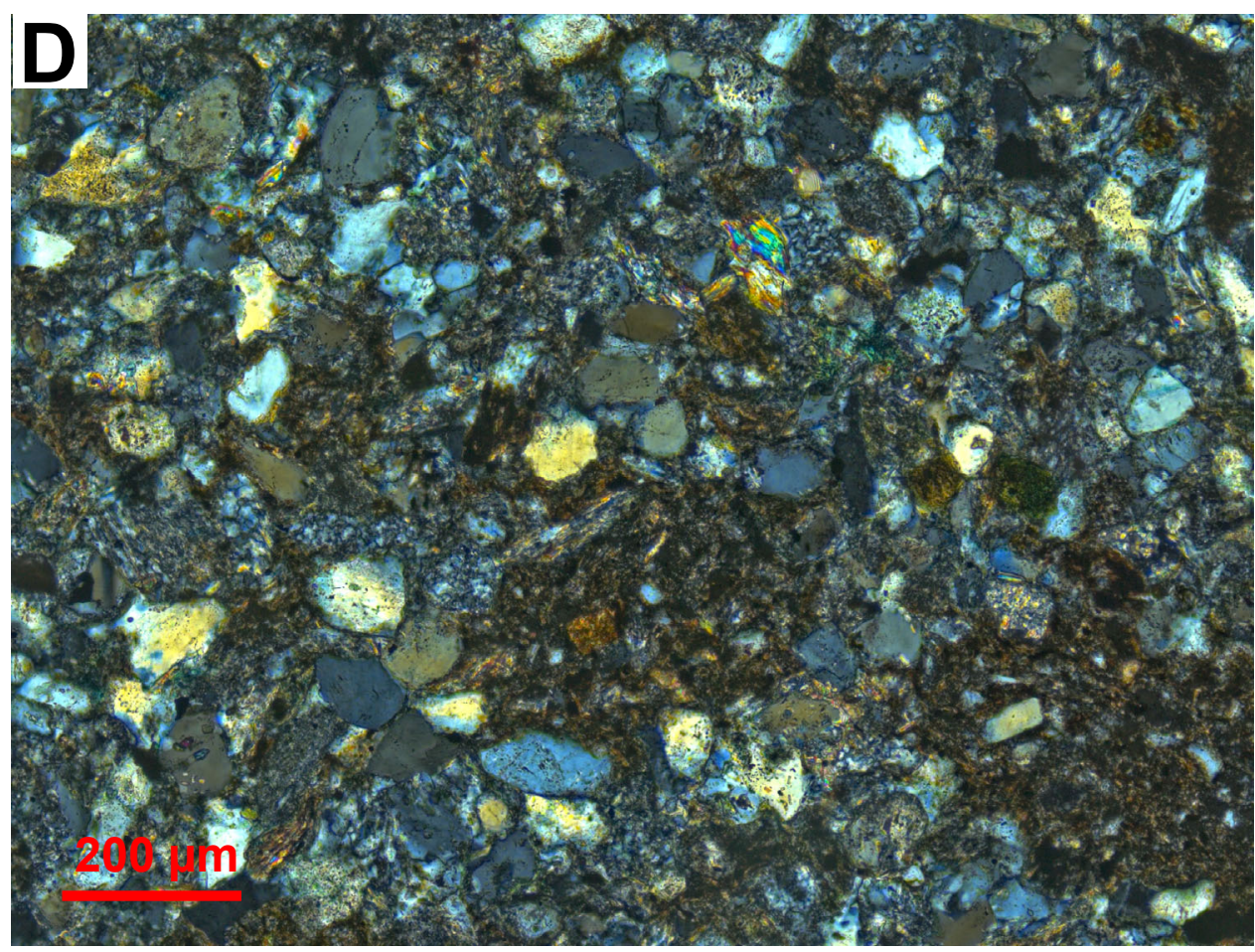




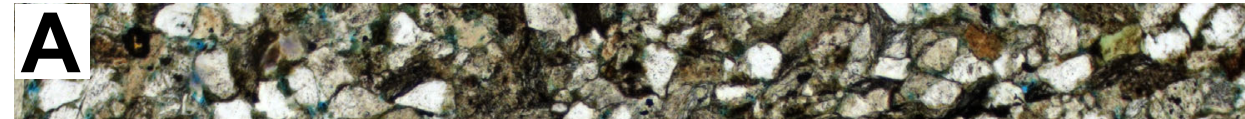

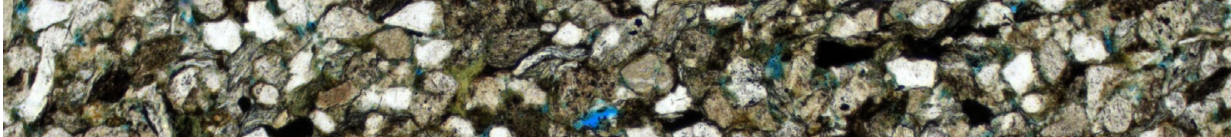

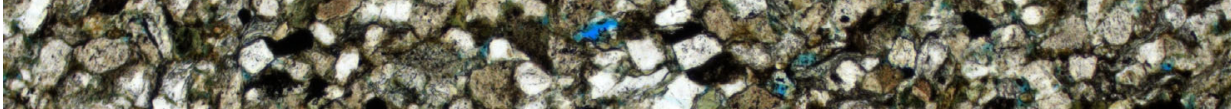
(1) 16

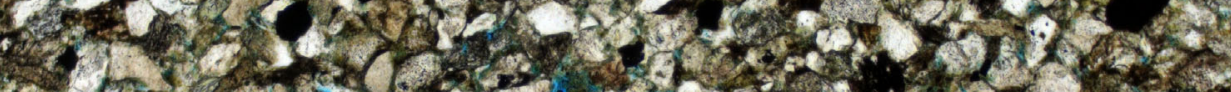

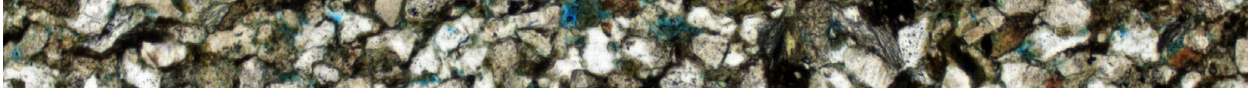

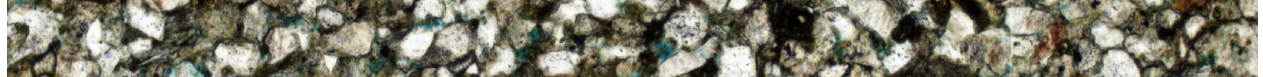

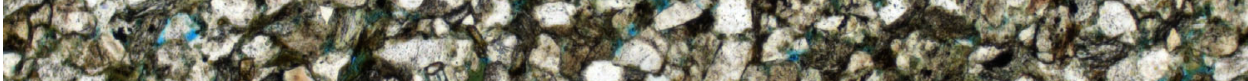

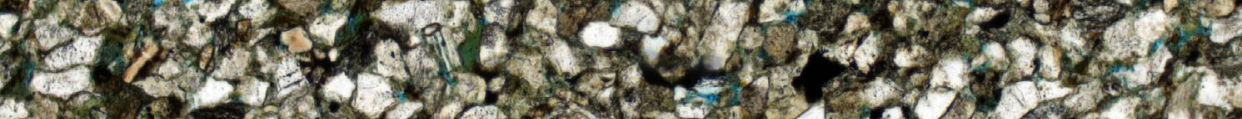

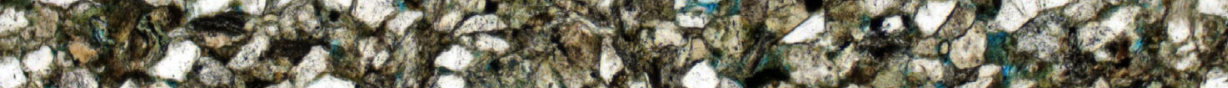

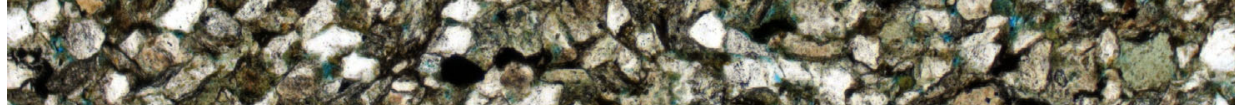

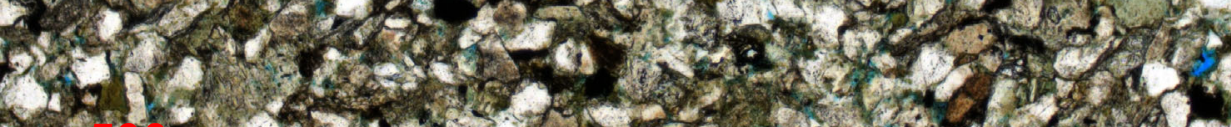

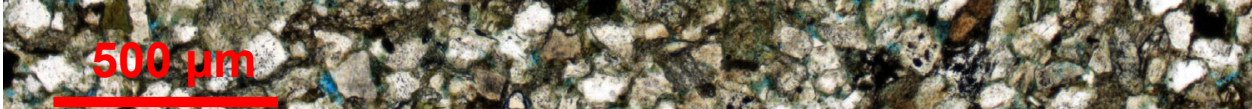

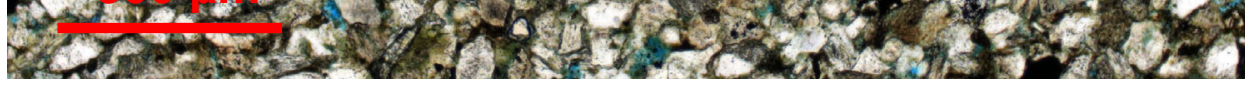
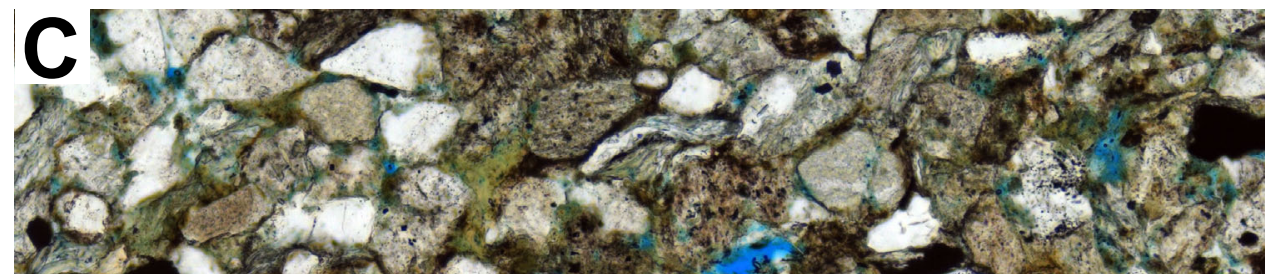

2

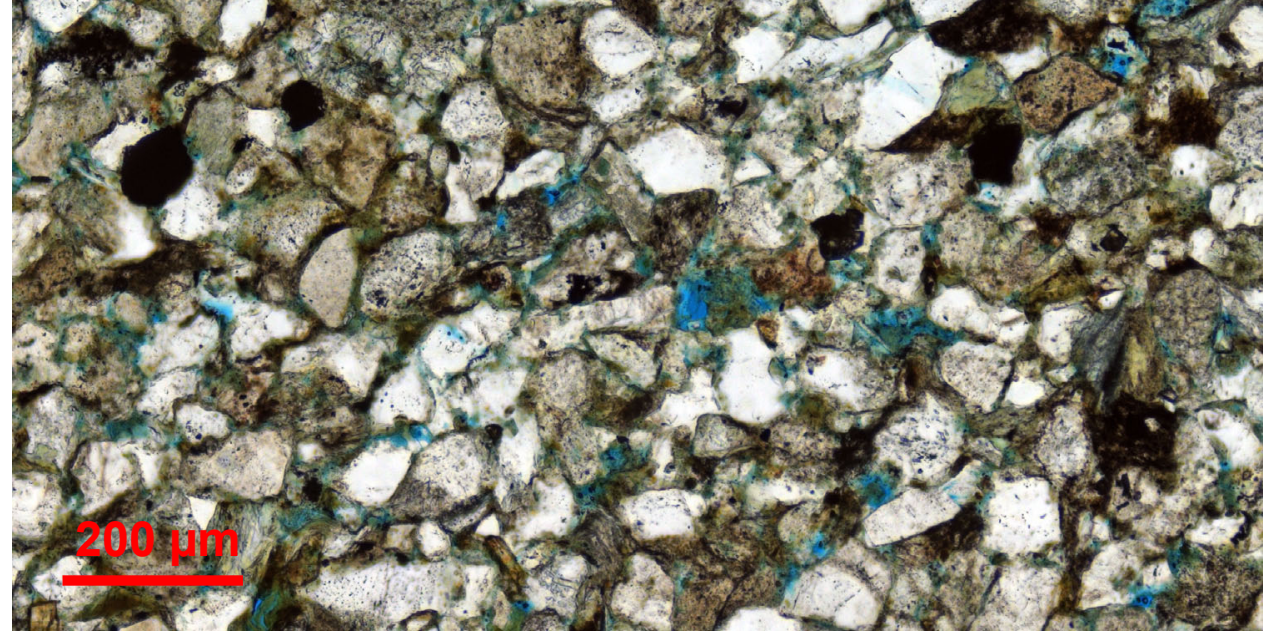

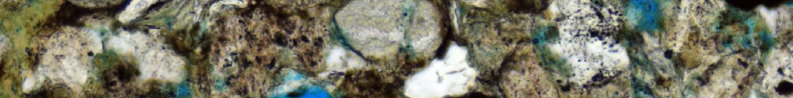

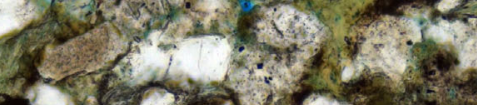
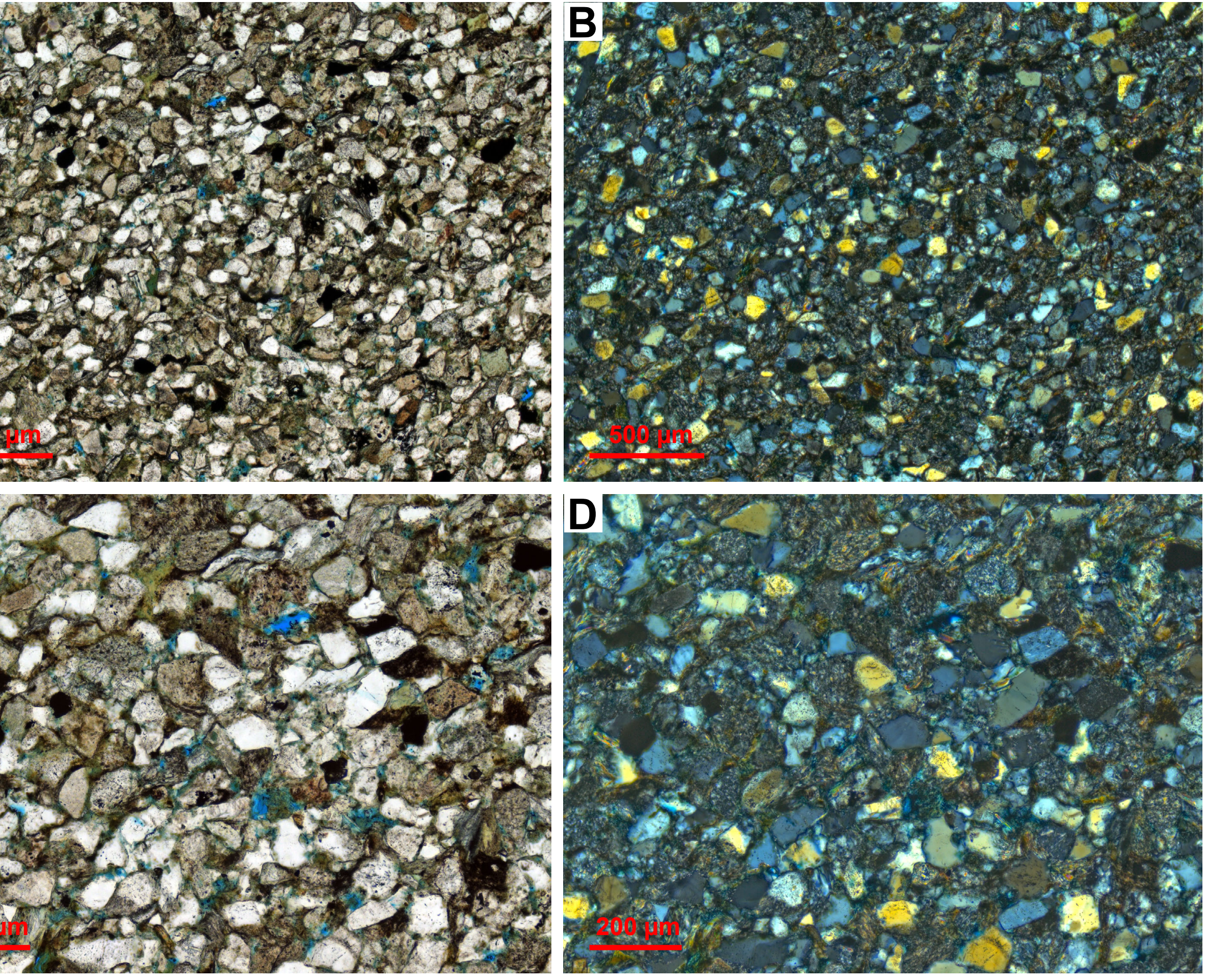
Fig $073-19 B G 002 A$
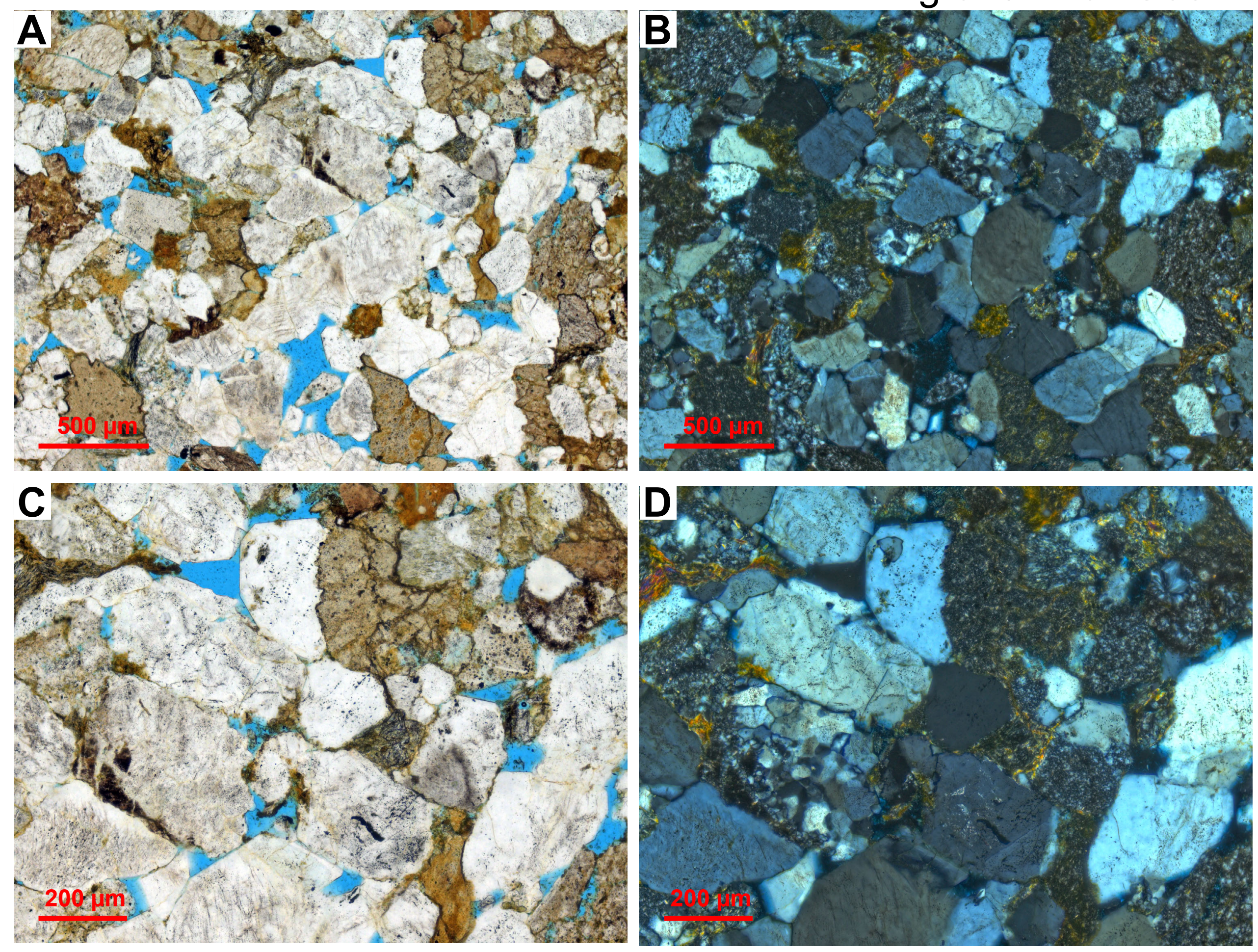
Fig 074 - 19DL008-001.0A
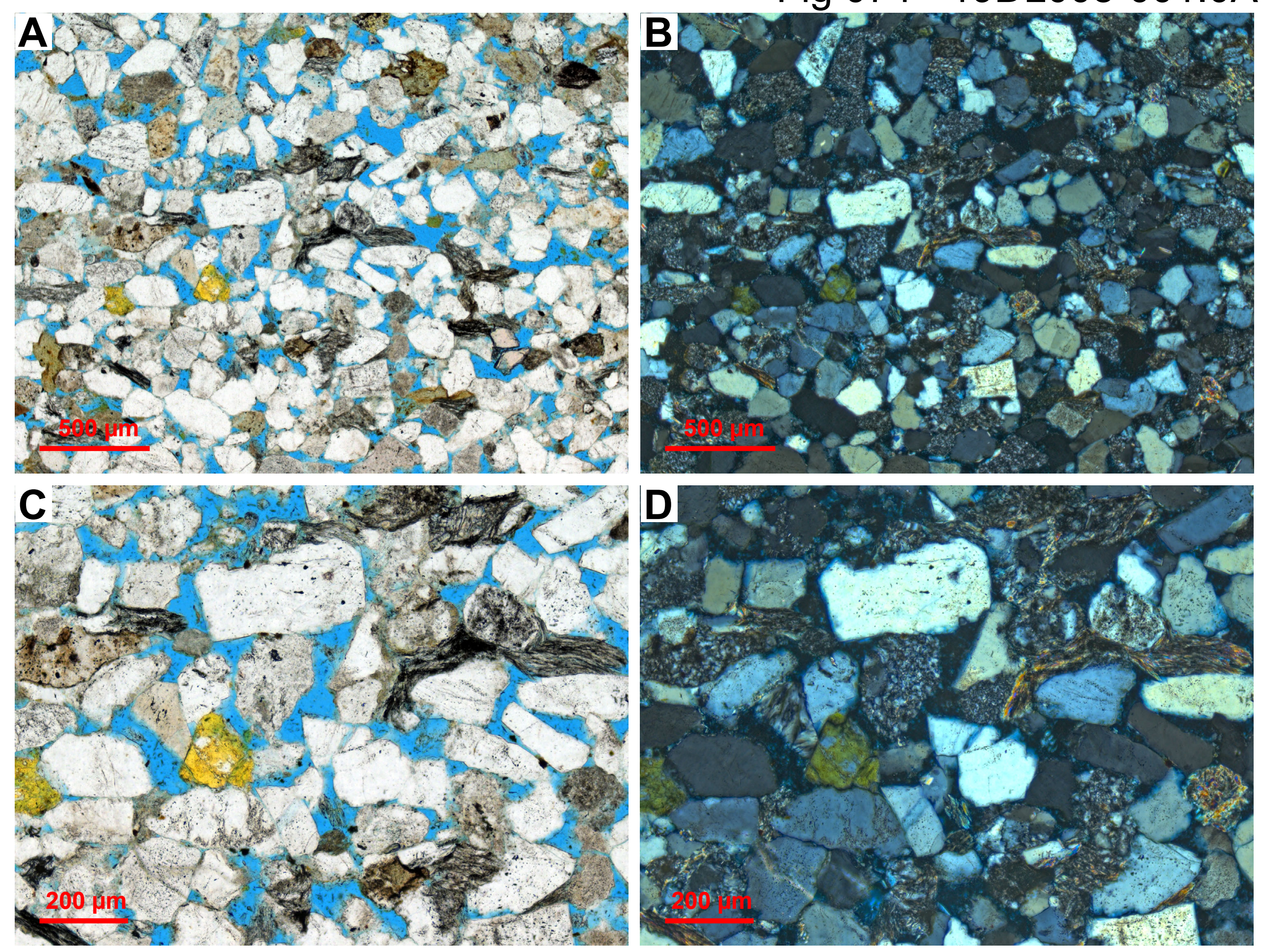
Fig 075 - 19DL008-015.0A

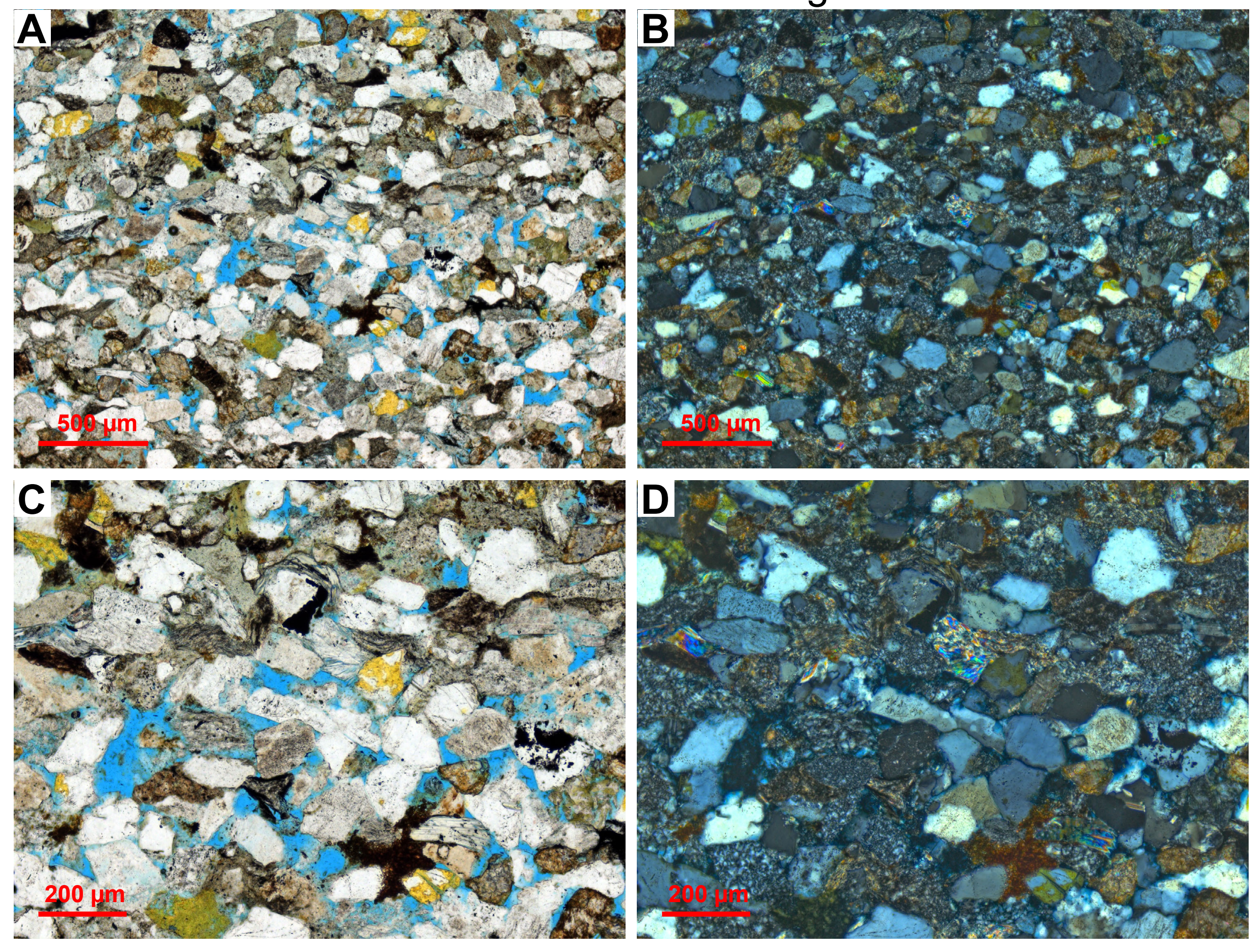


Fig 076 - 00DL016-000.1

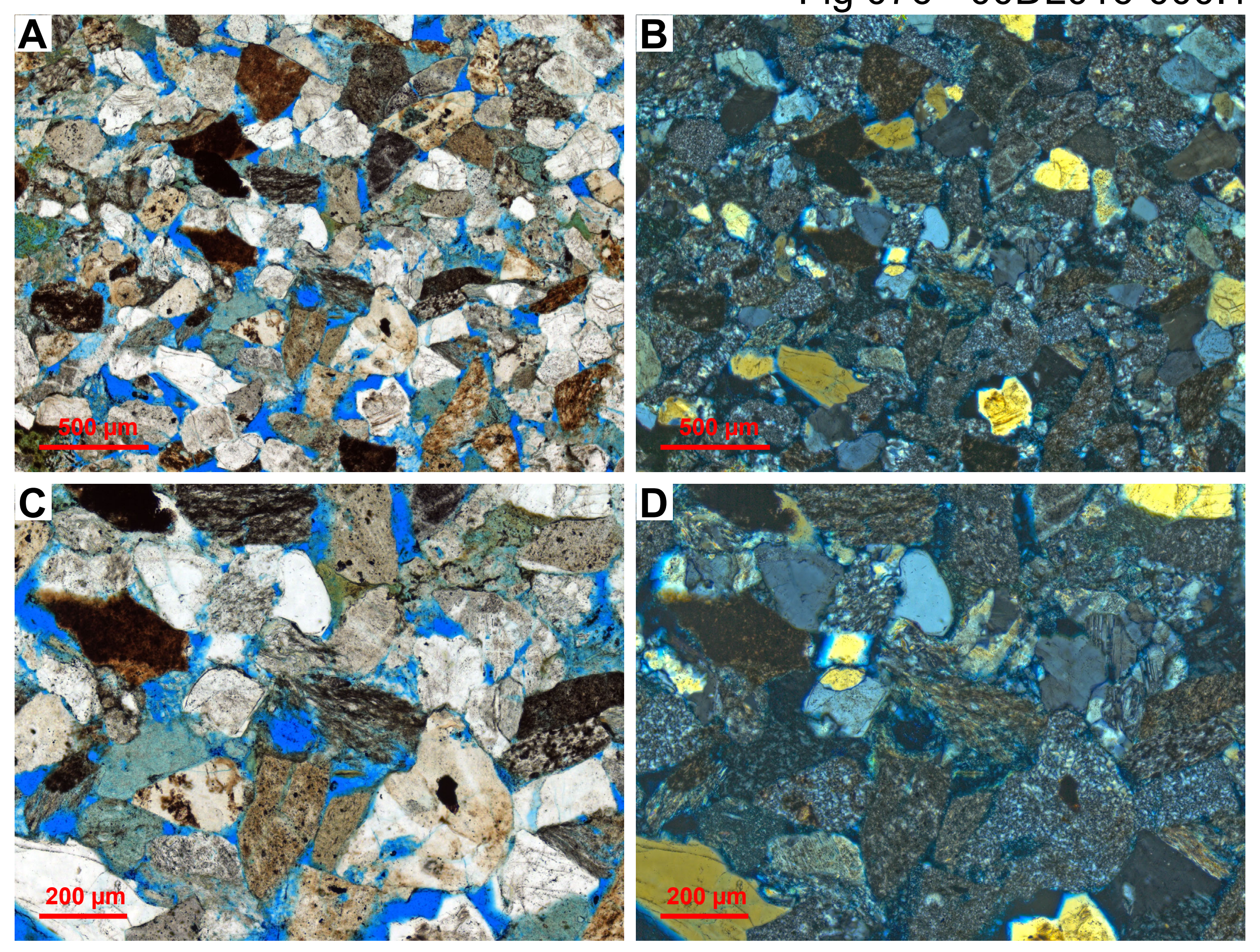


Fig 077 - 00DL016-039.7
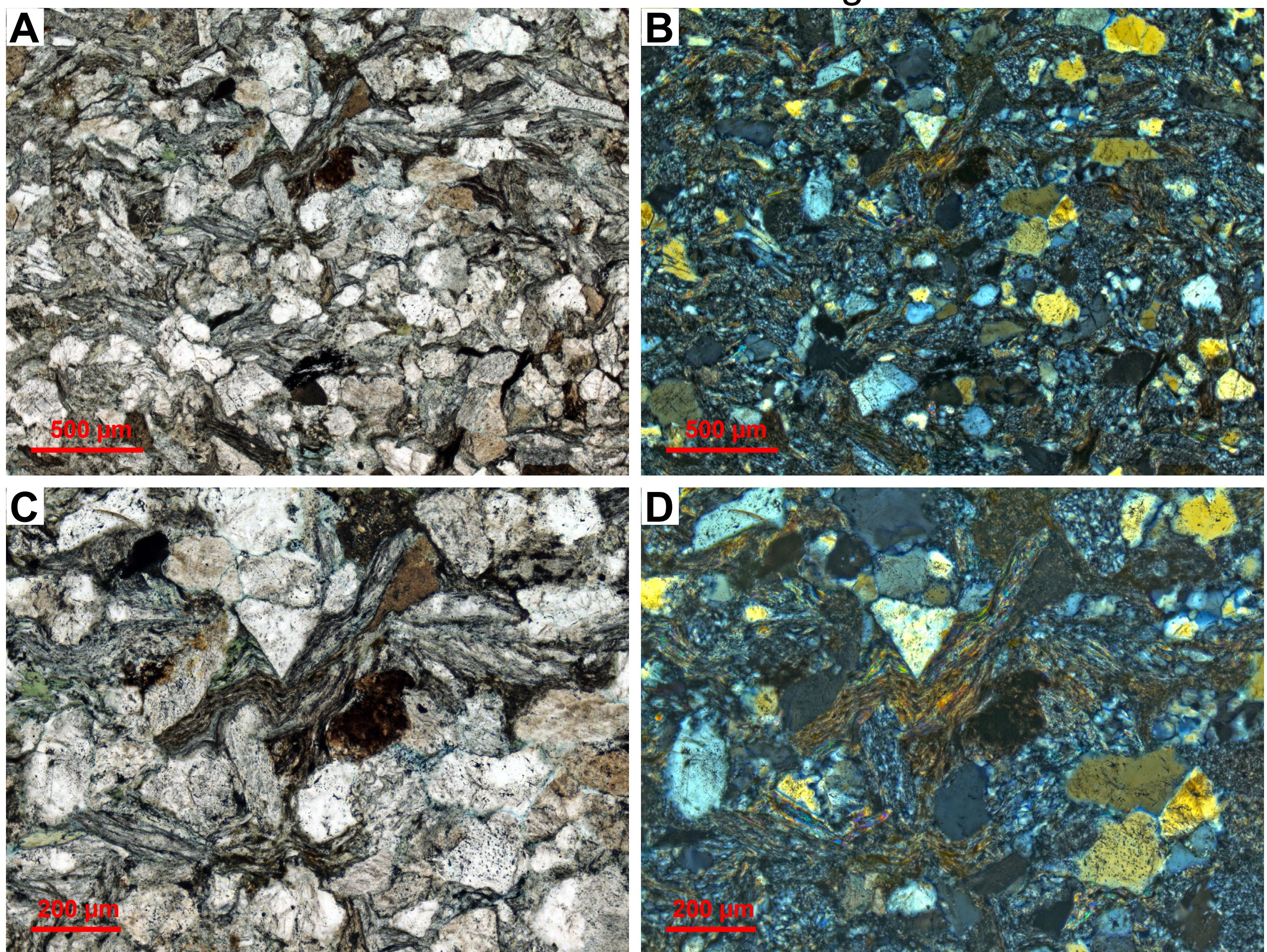
Fig 078 - 00DL016-082.0
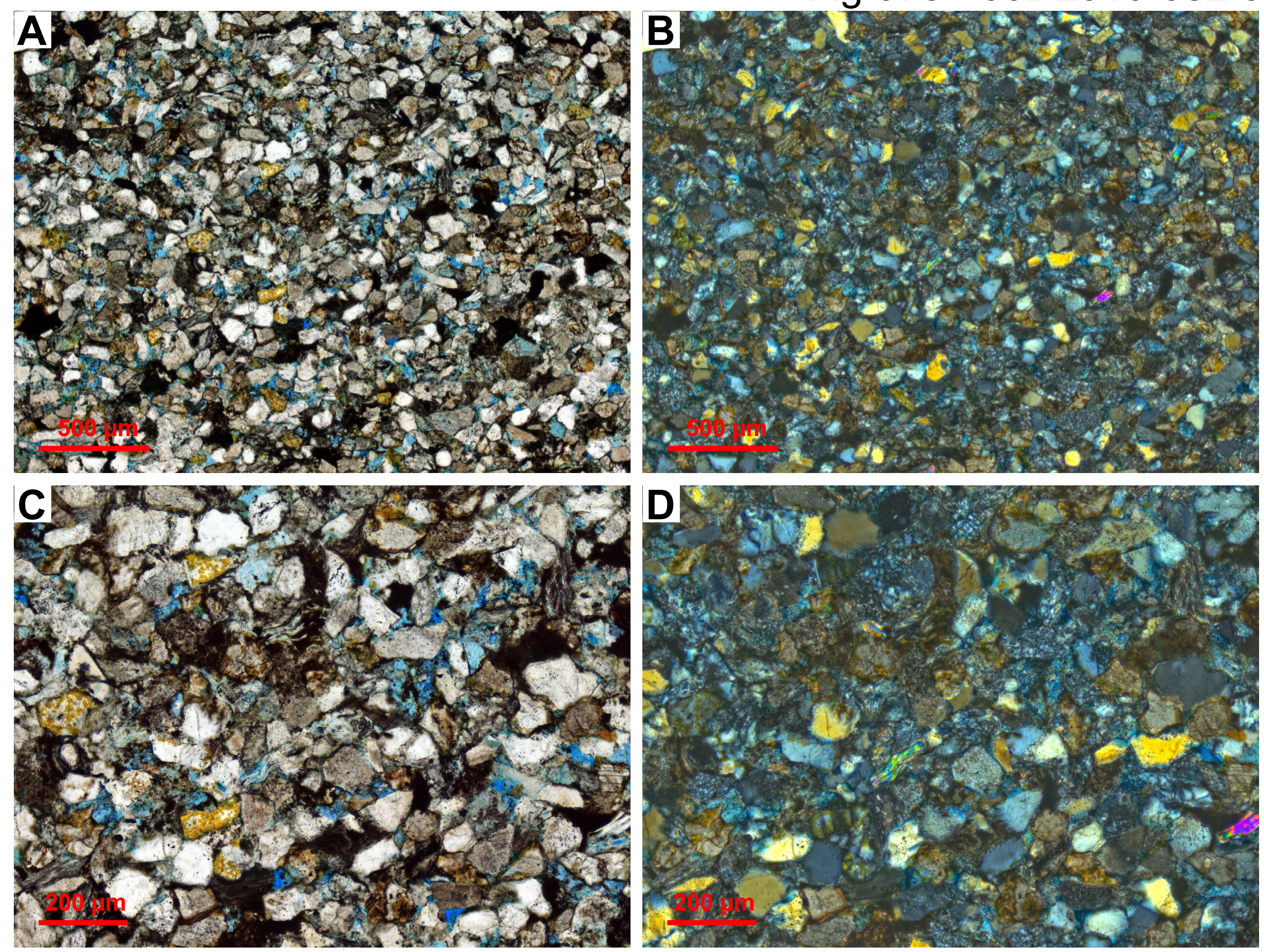
Fig 079 - 00DL016-086.0
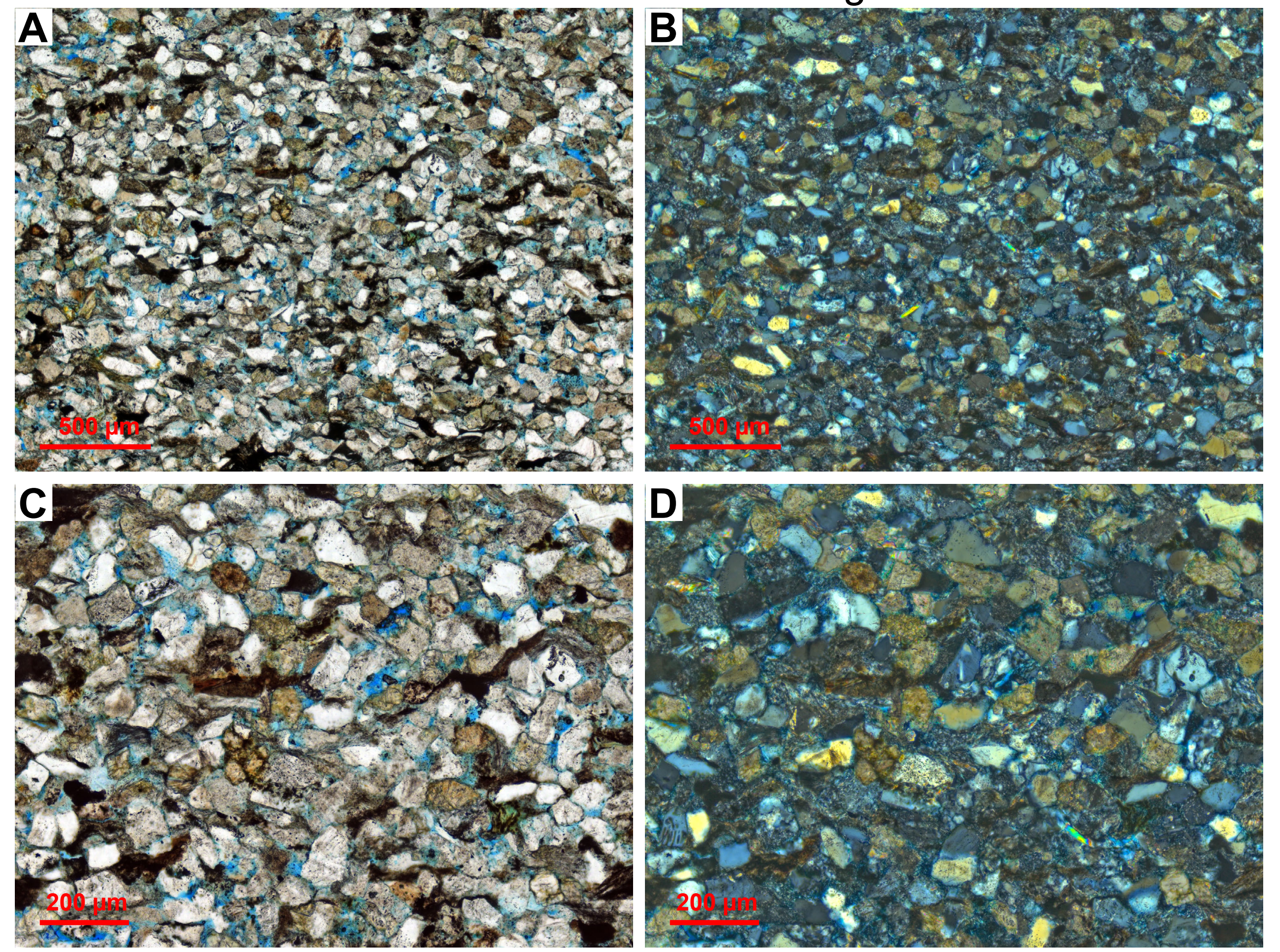

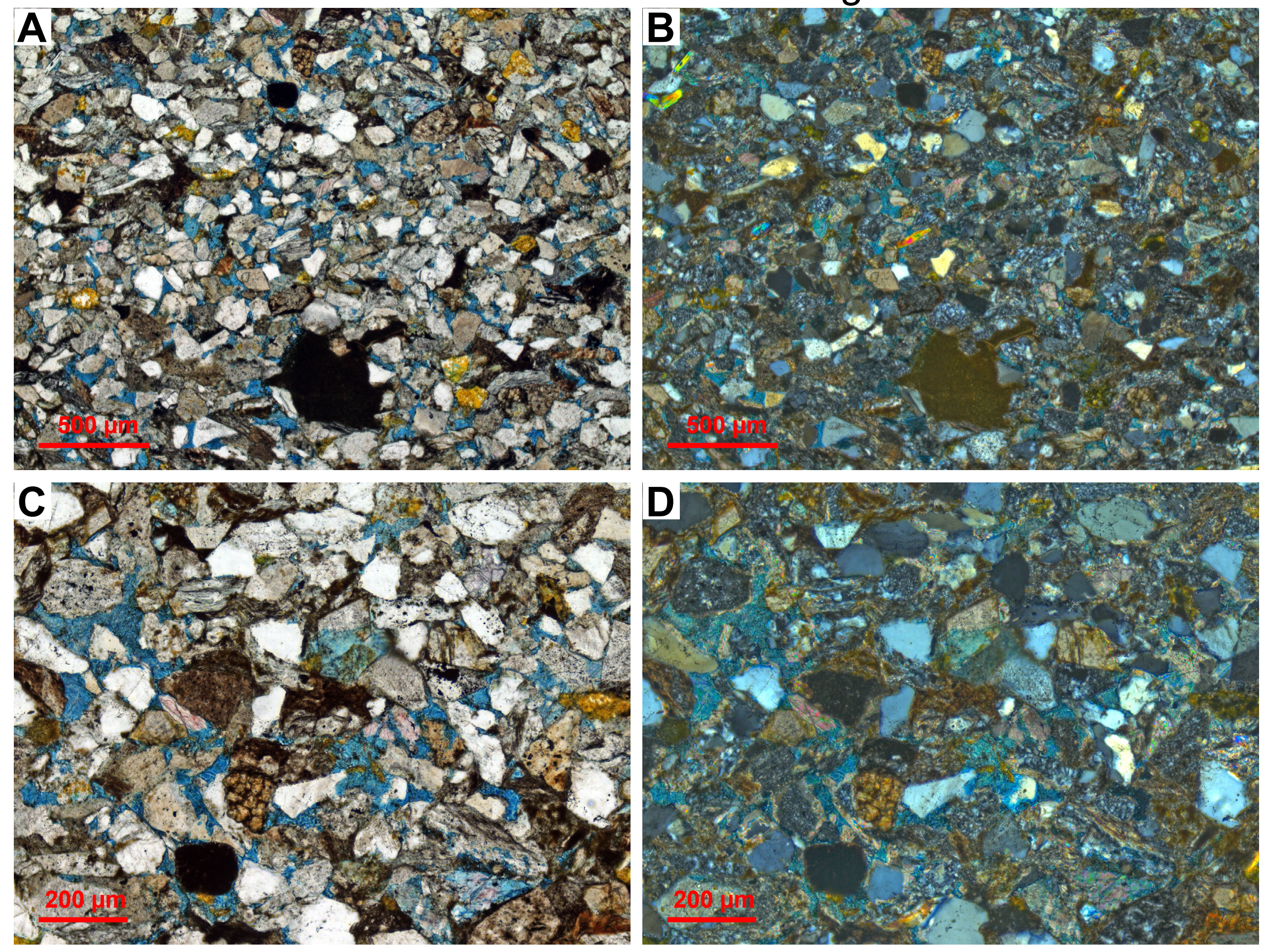
Fig 081 - 00DL016-090.0

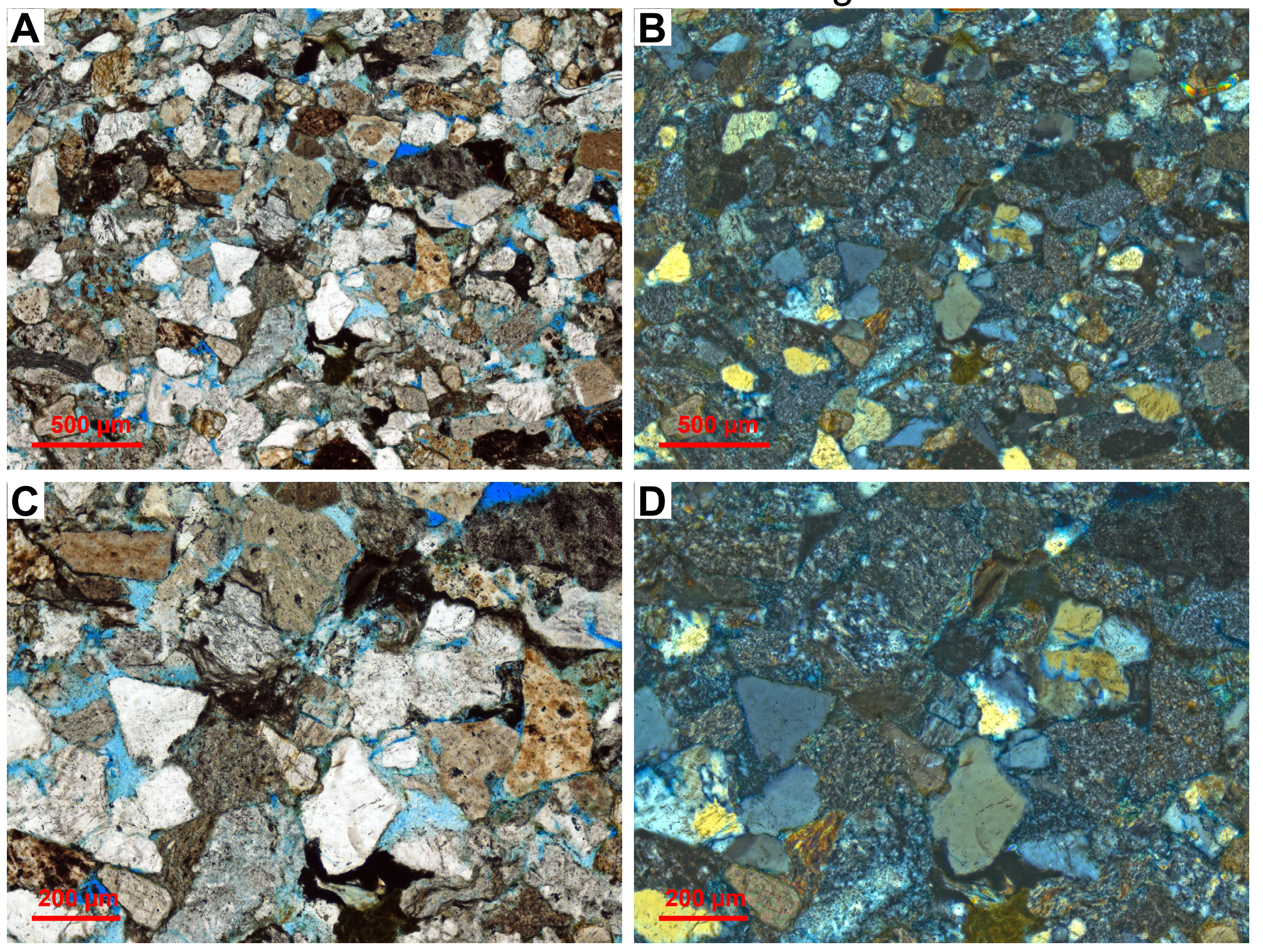


Fig 082 - 00DL016-092.0

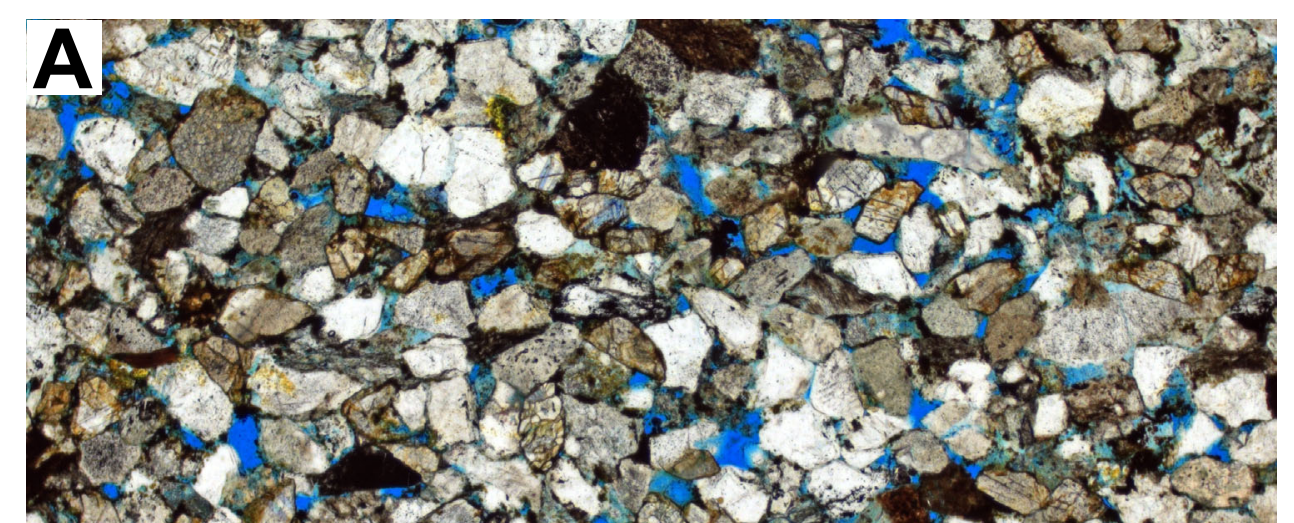
ingra 3 ,

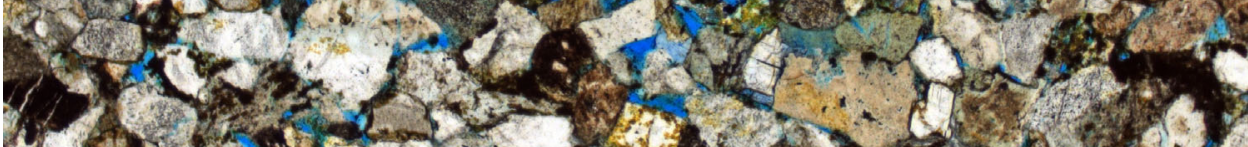

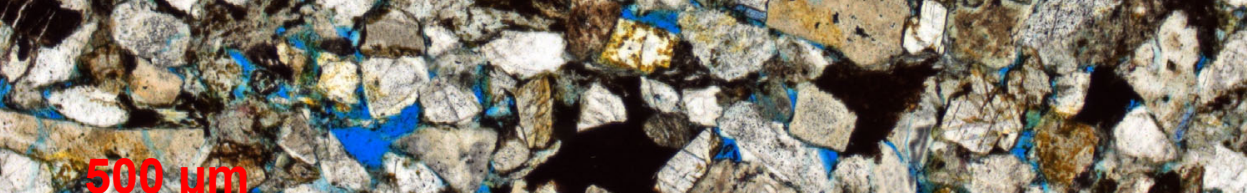

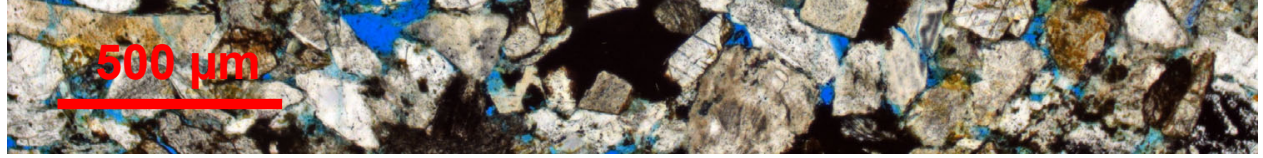
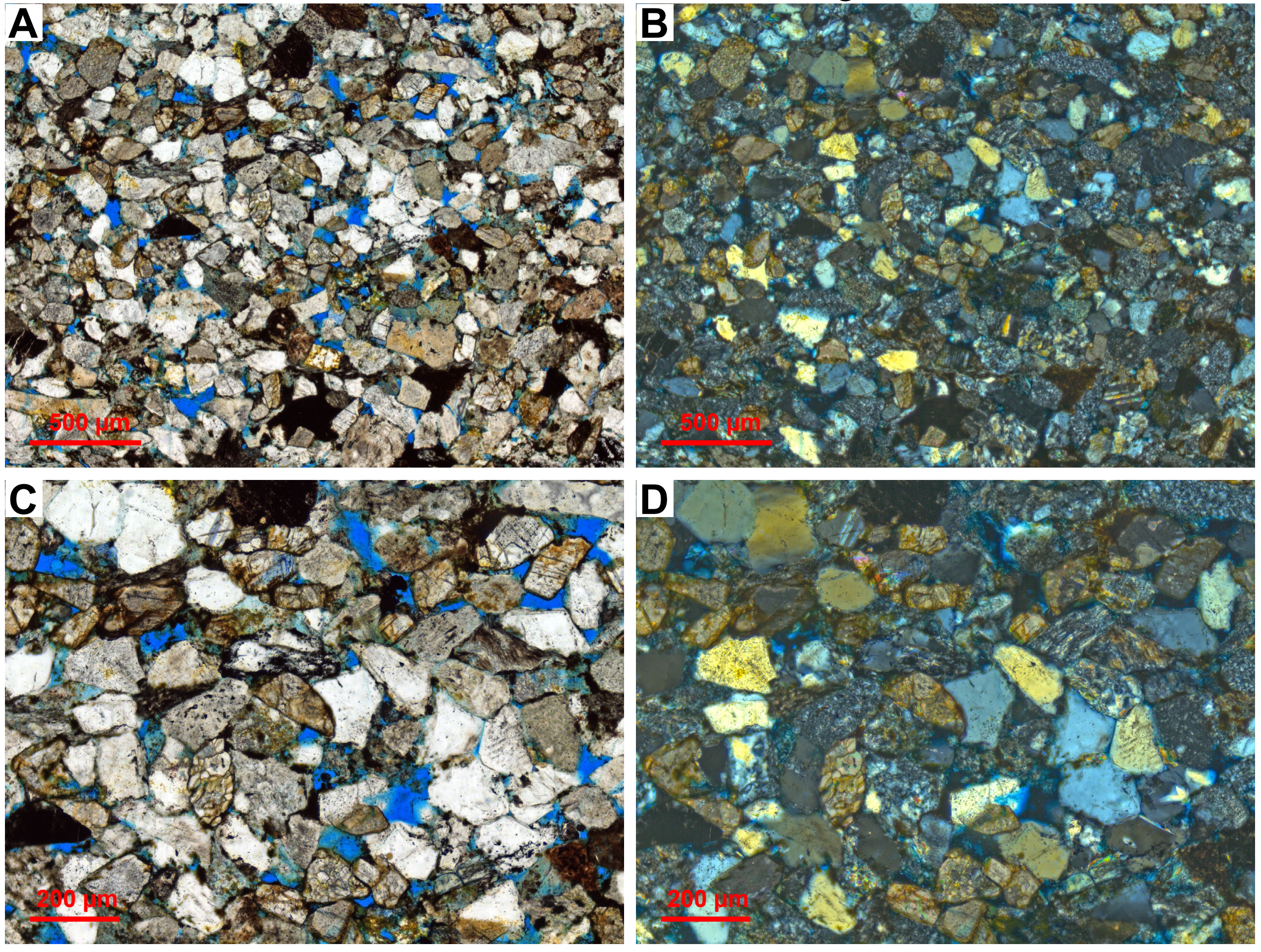
Fig 083 - 00DL016-122.0
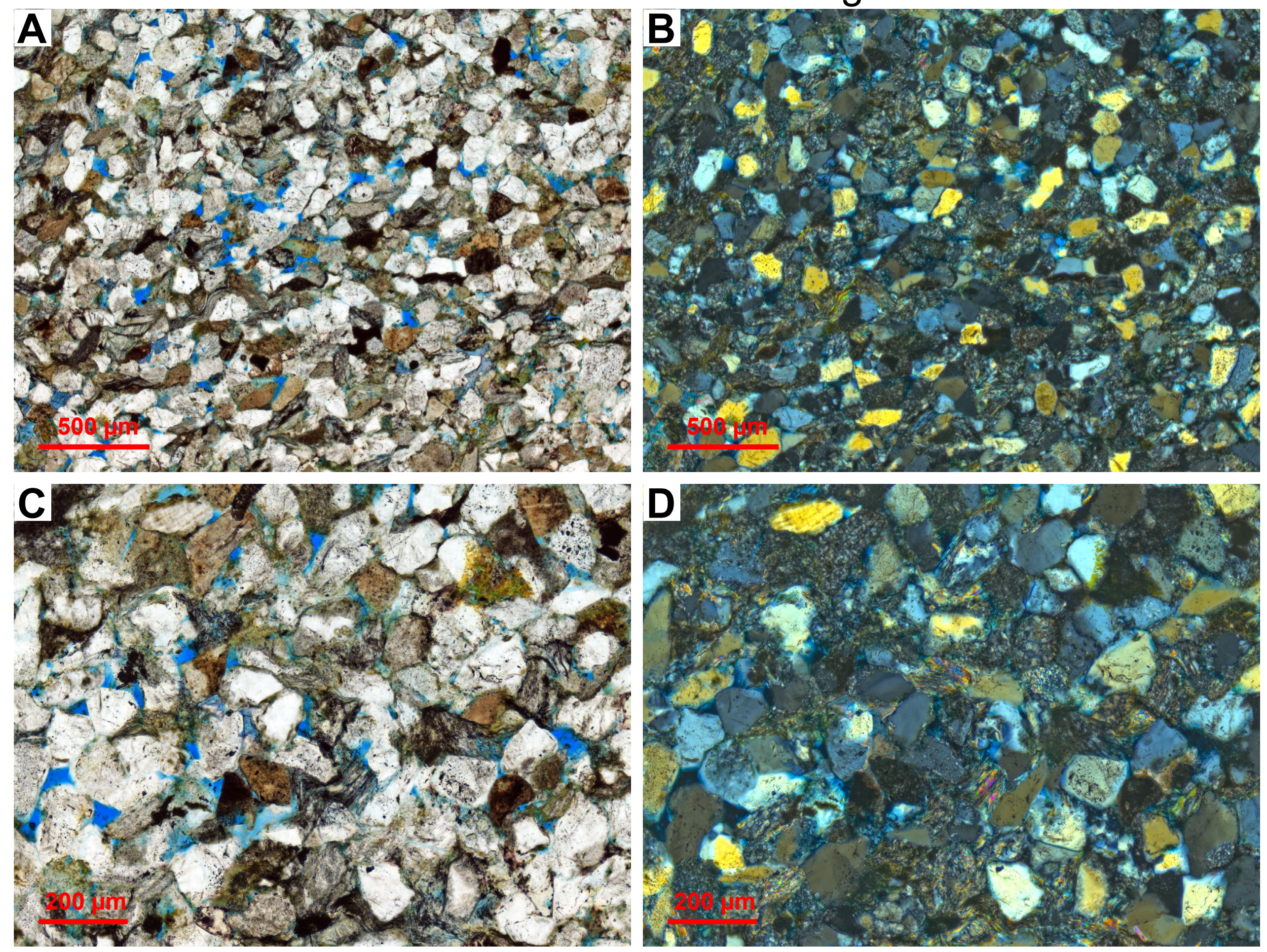
Fig 084 - 00DL016-125.0
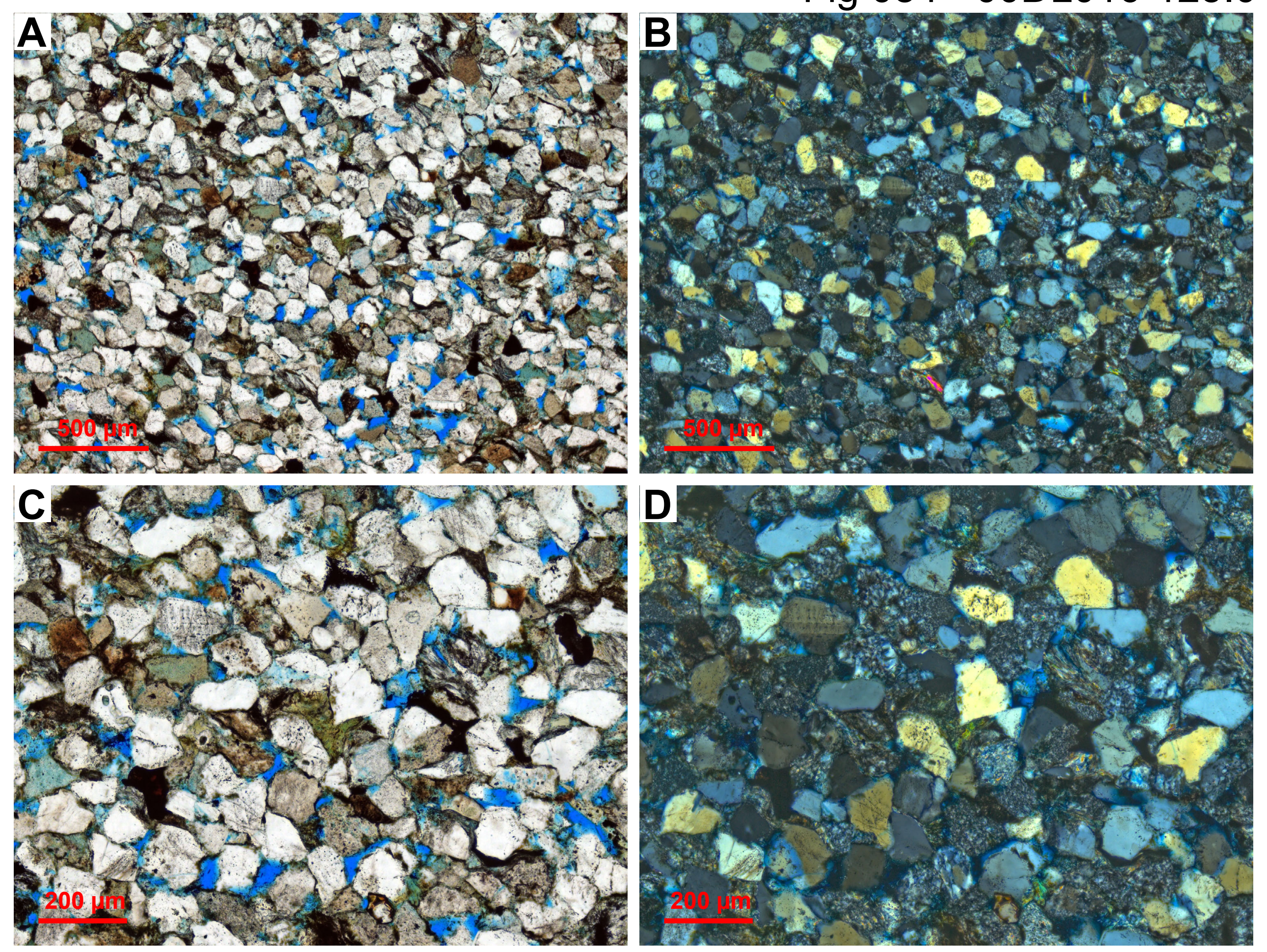
Fig 085 - 00DL016-133.0

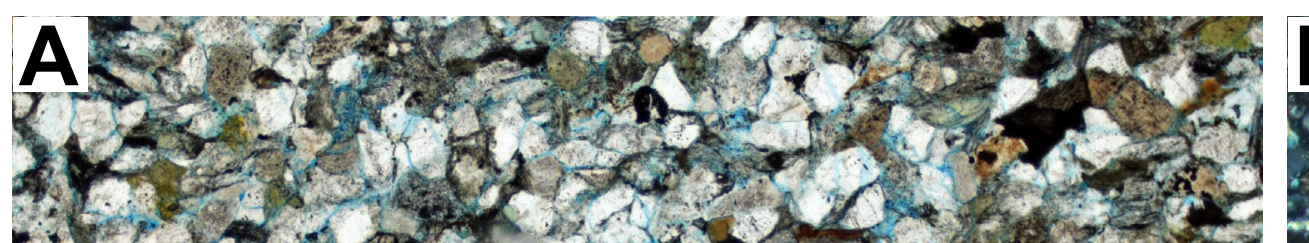

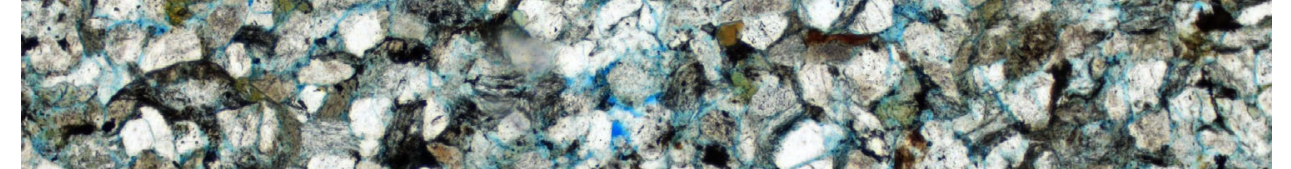

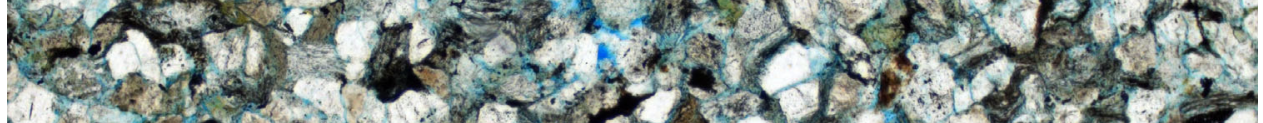

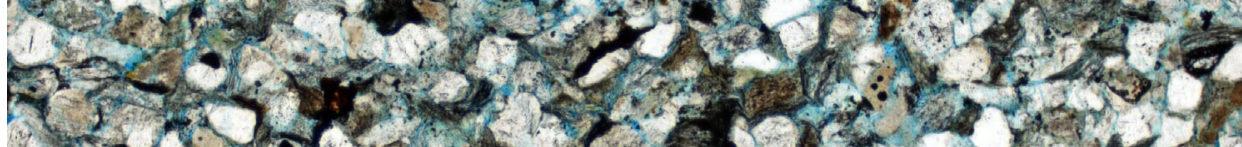

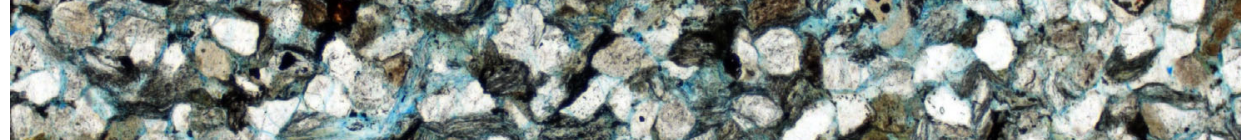

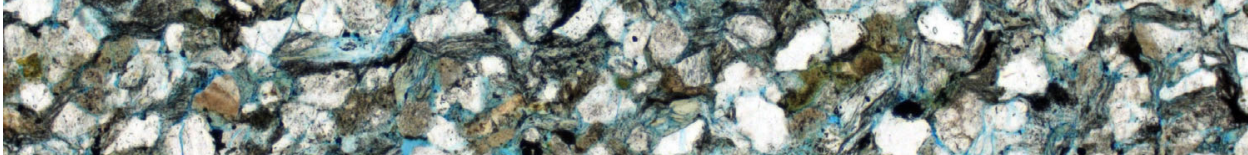

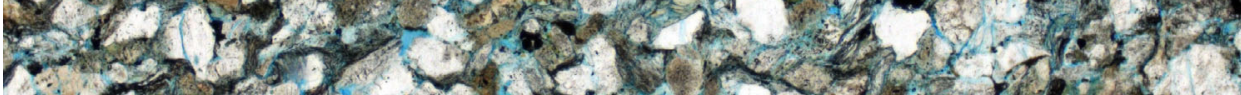

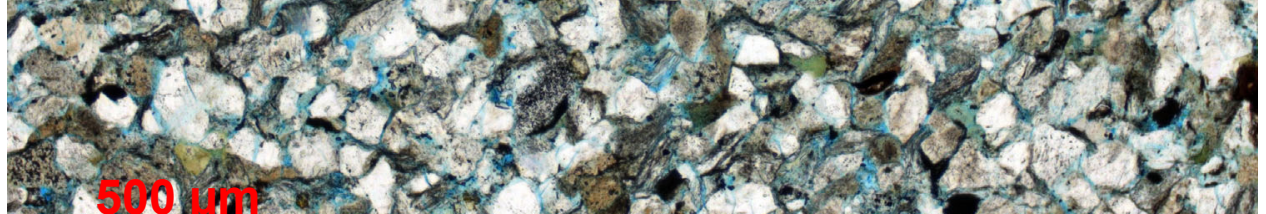

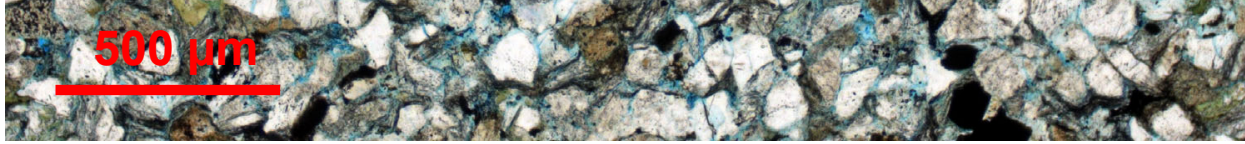
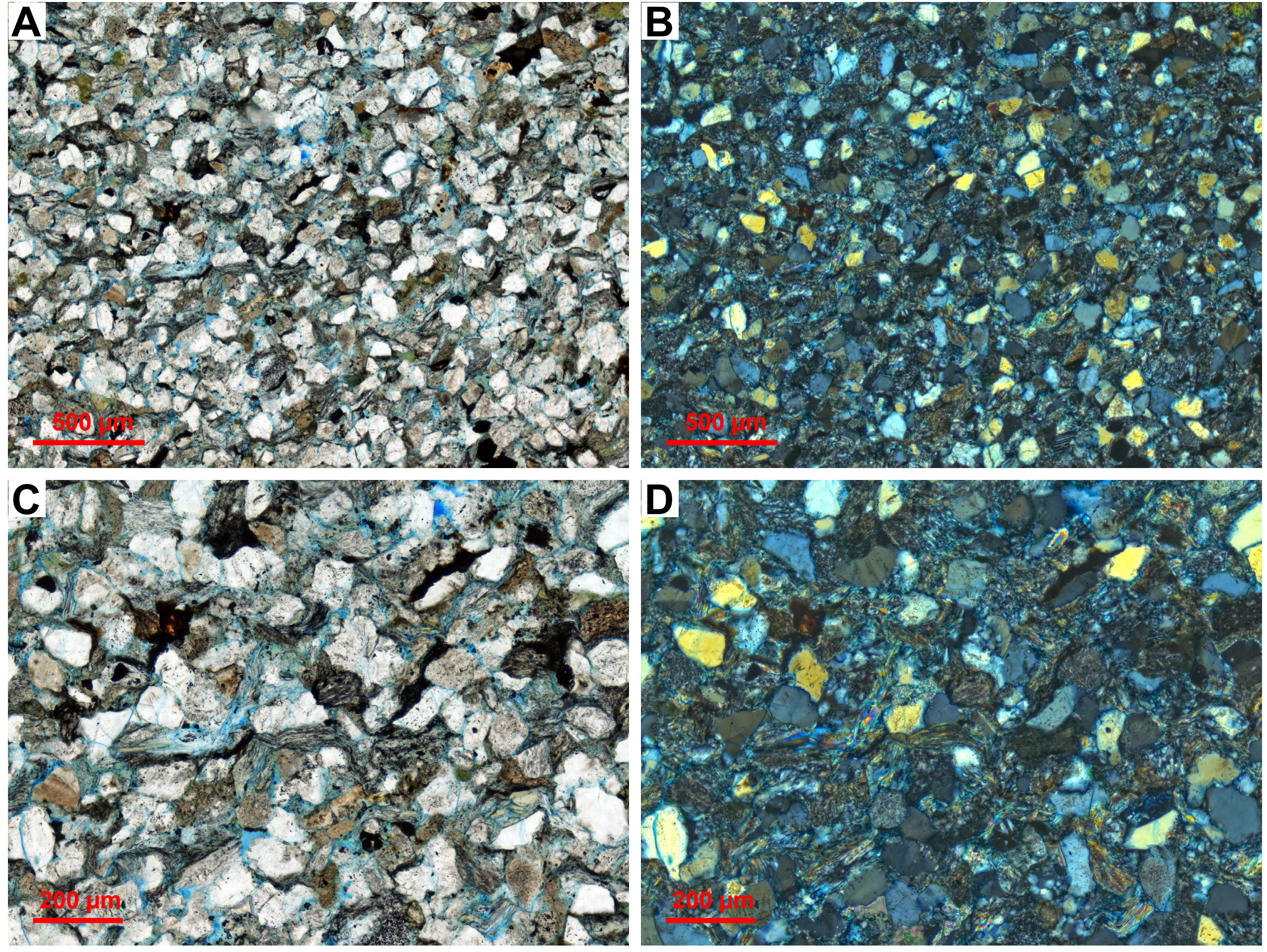
Fig 086 - 00DL016-135.0
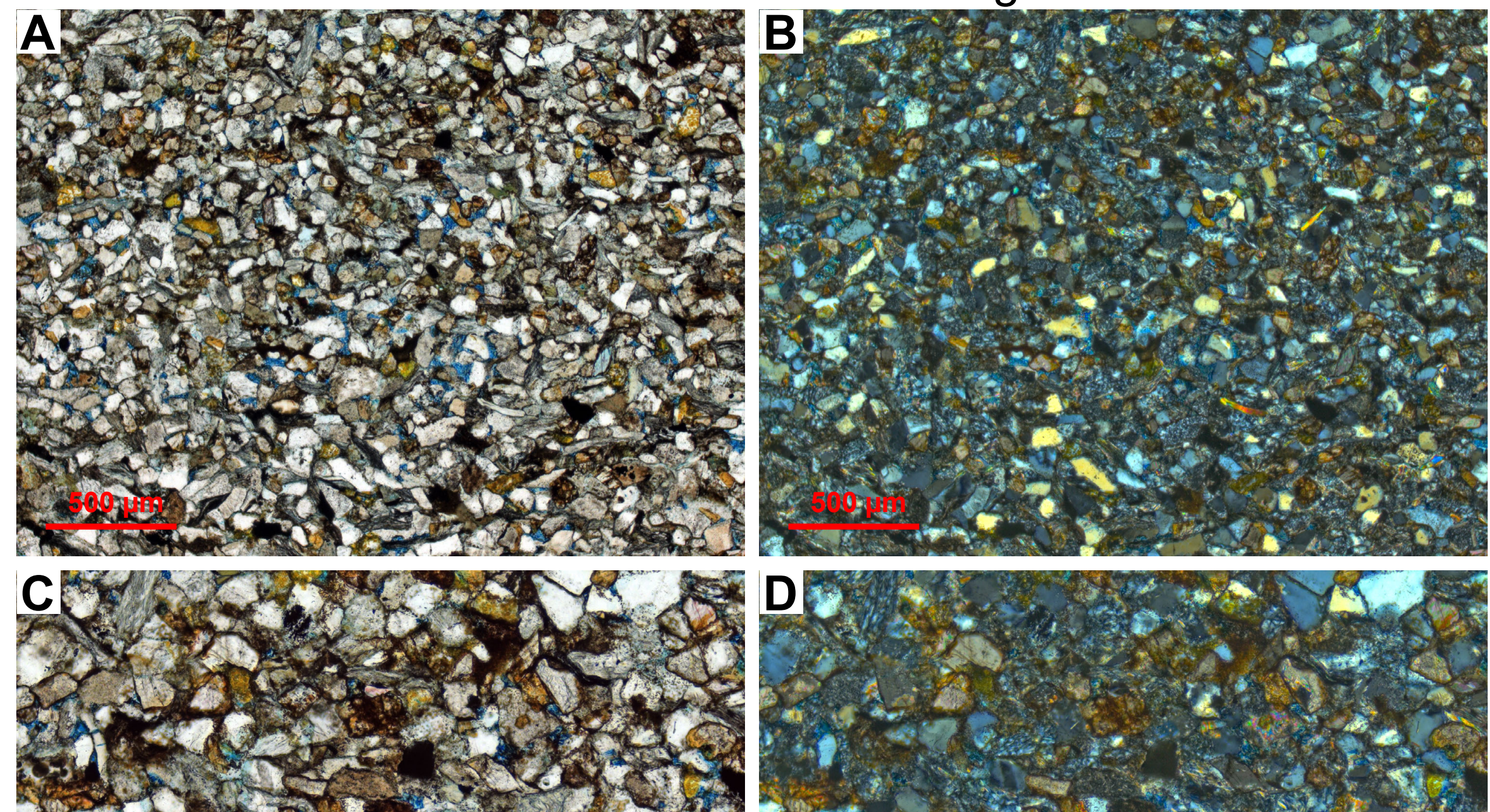
$10 \times 5 \times 17$ I 1,21210 1.6.

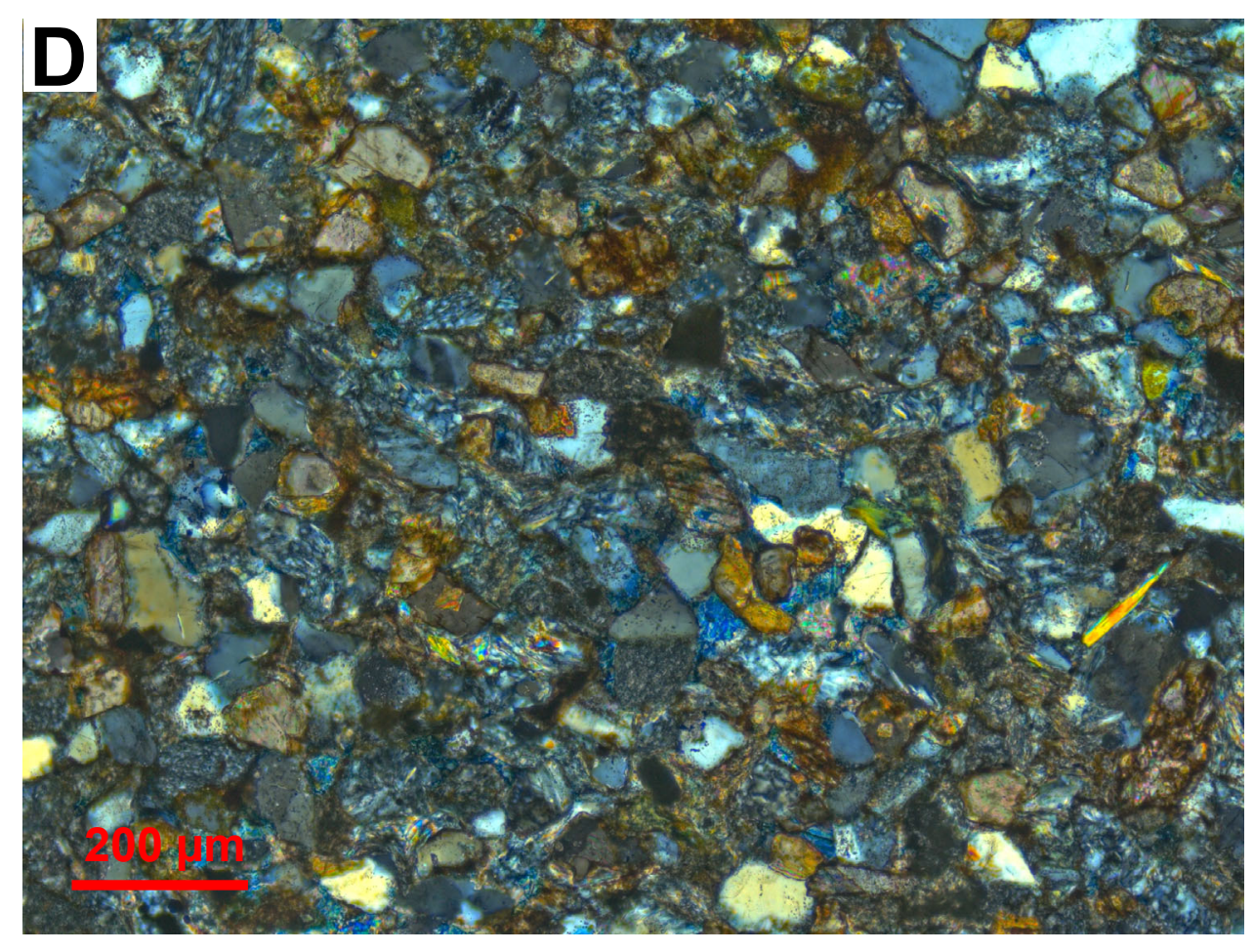




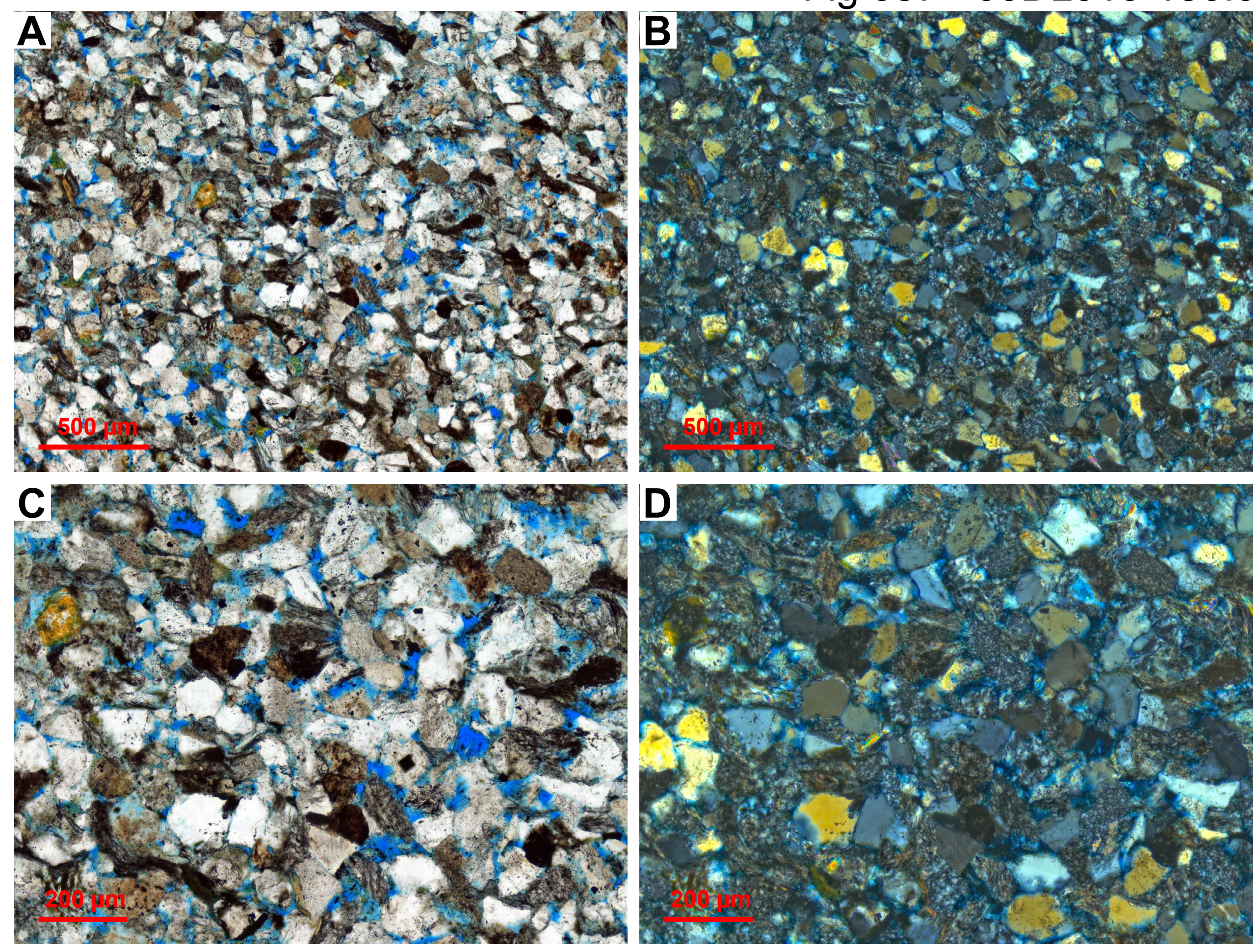


Fig 088 - 00DL016-208.65
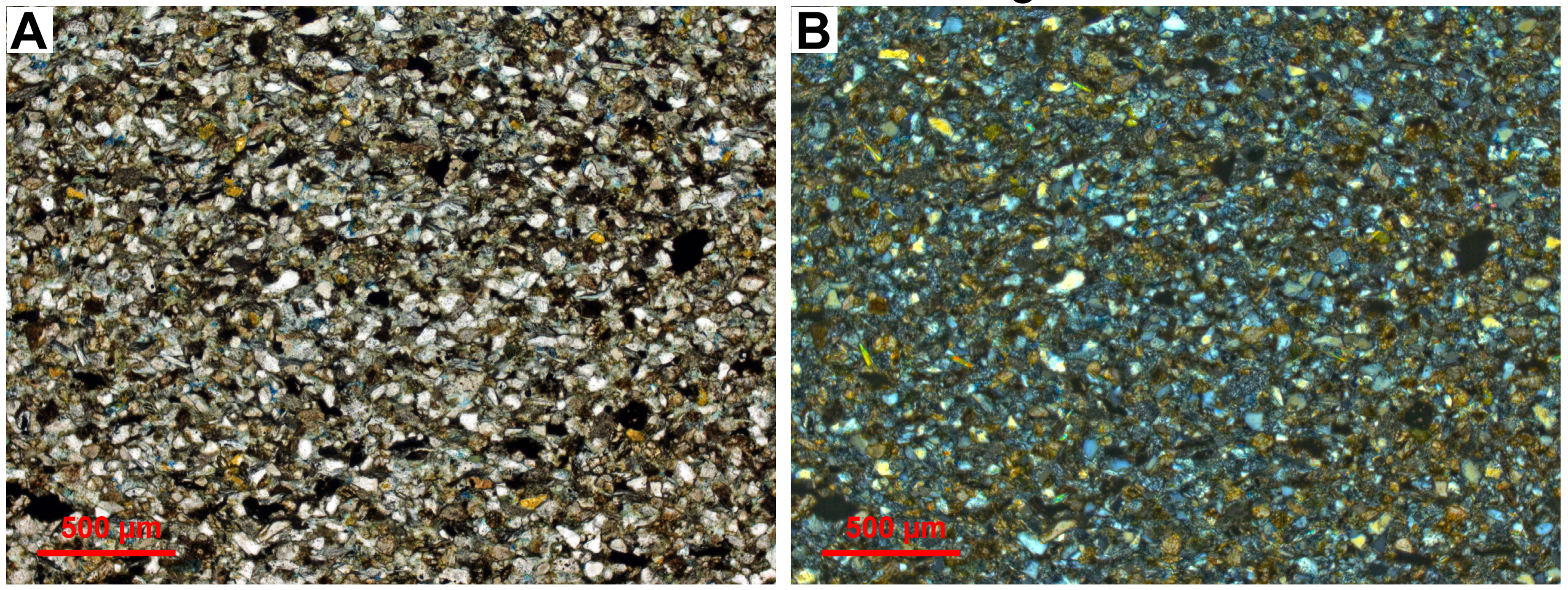

\section{CE} . .
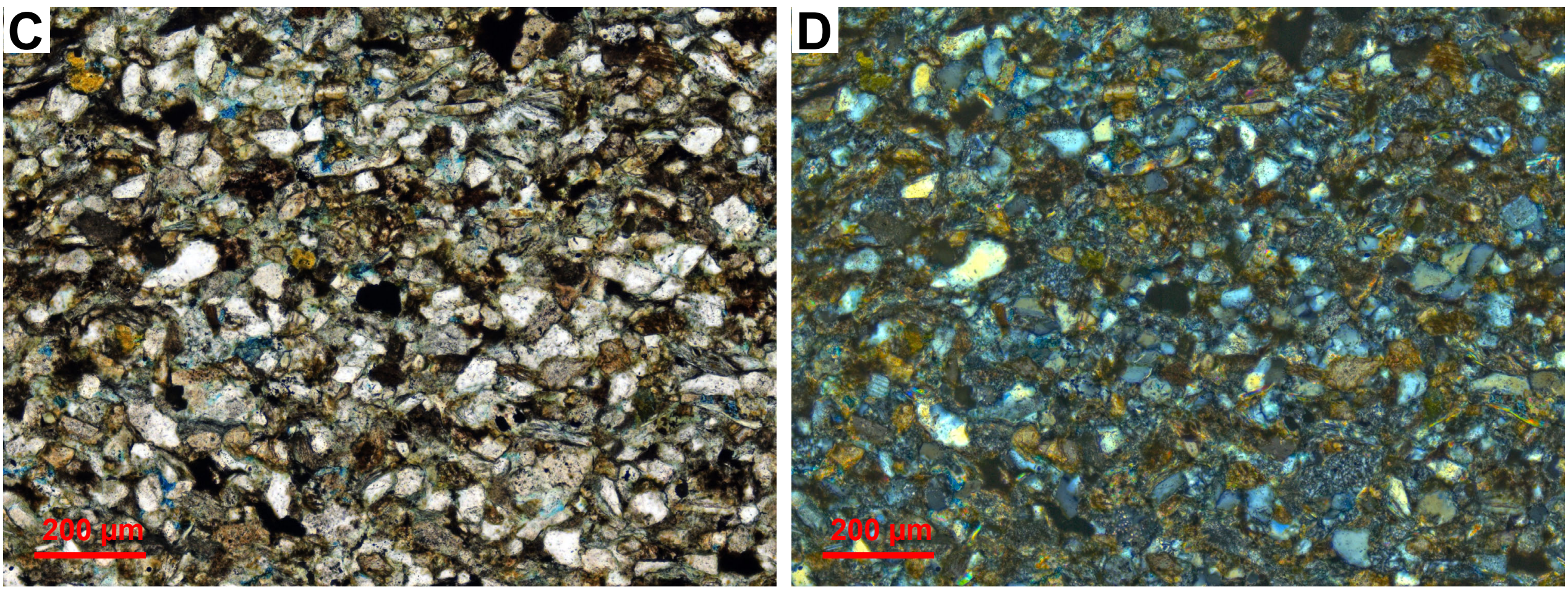
Fig 089 - 19DL026A

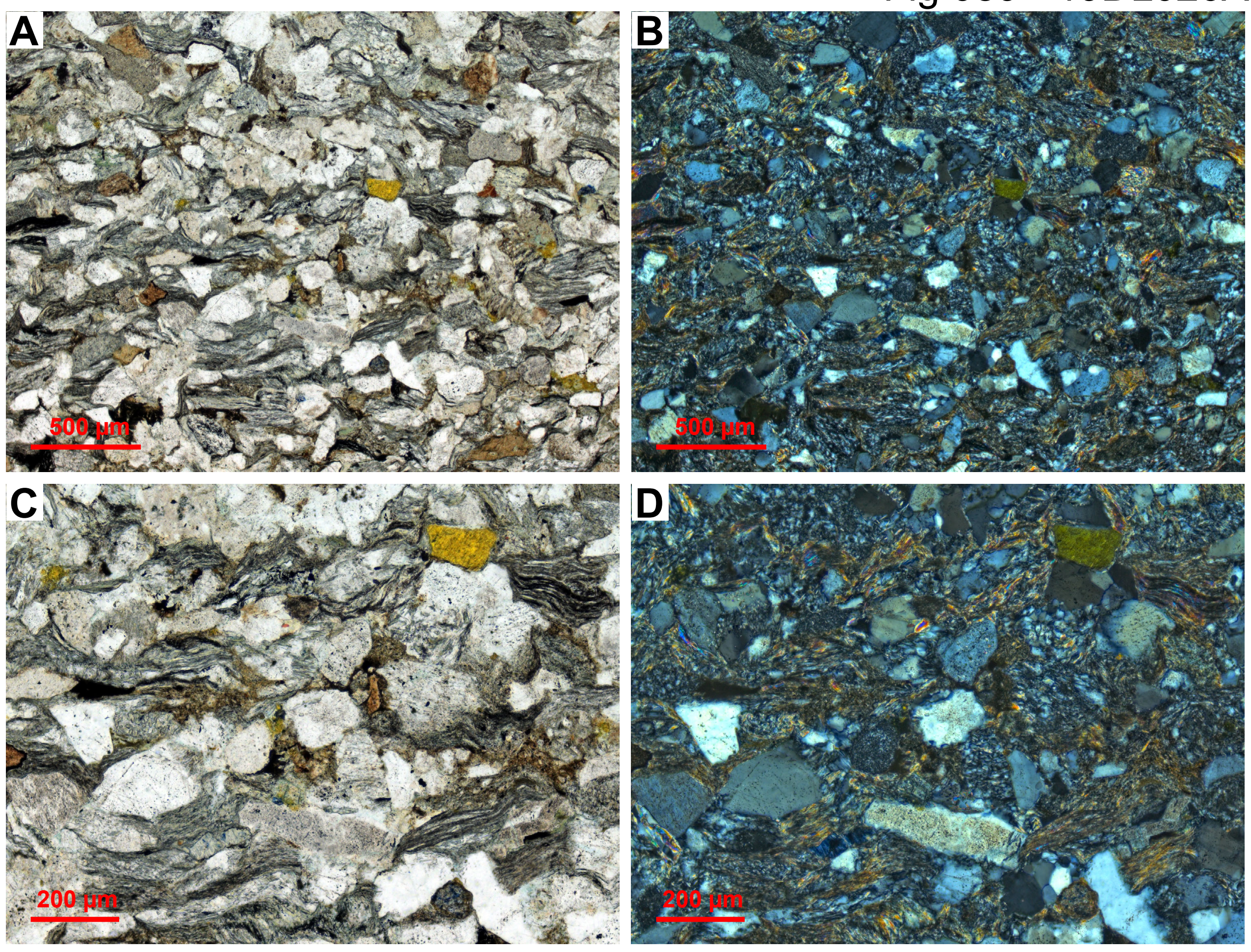


Fig 090 - 19DL028A

A fr. 10.7.

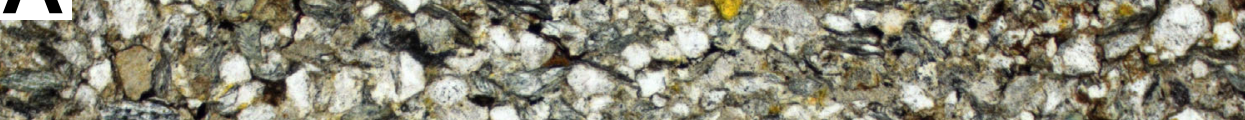
7 1.

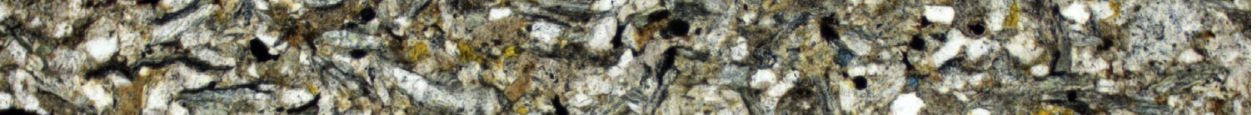

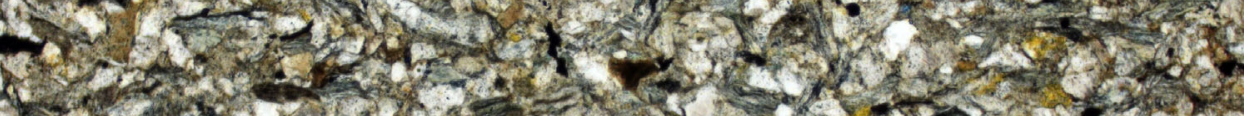

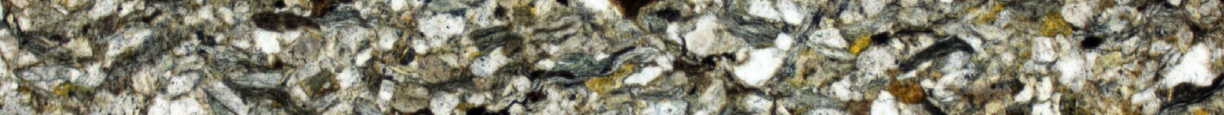

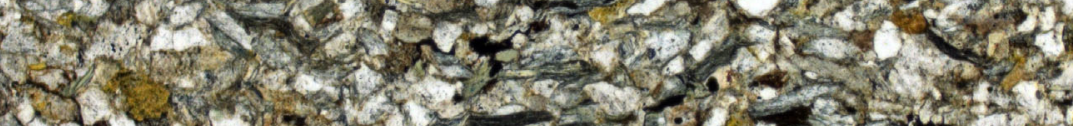

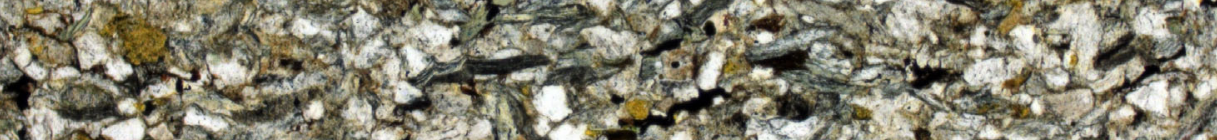

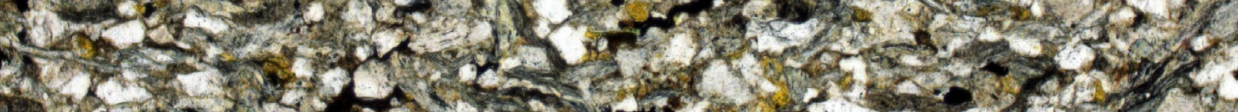

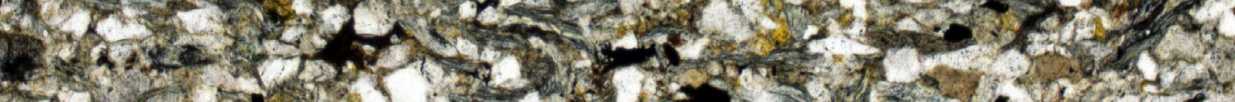

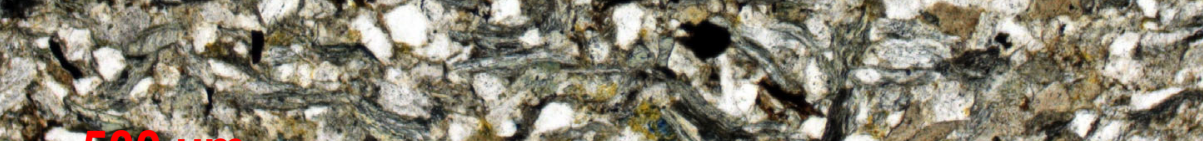

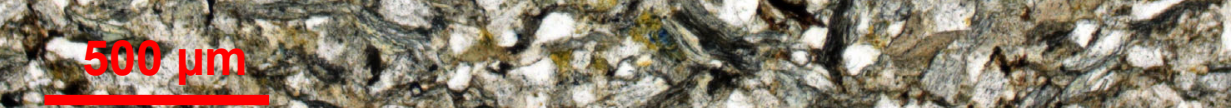

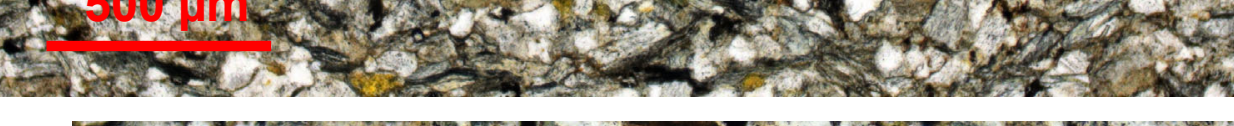

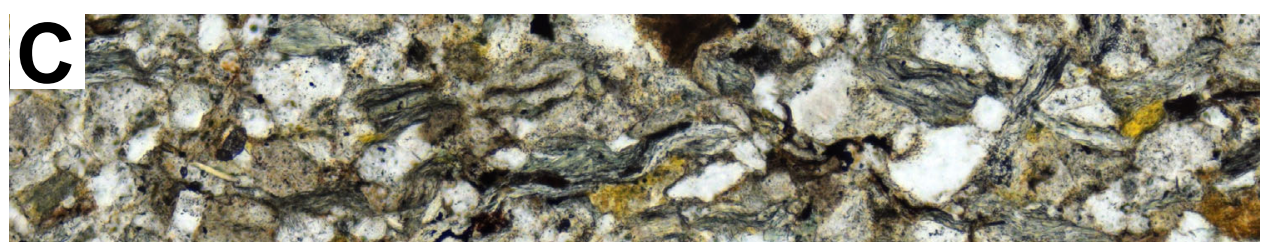

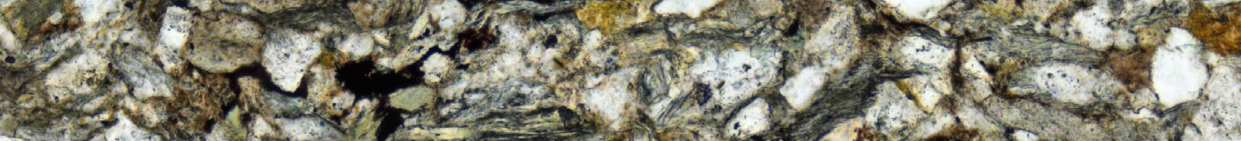

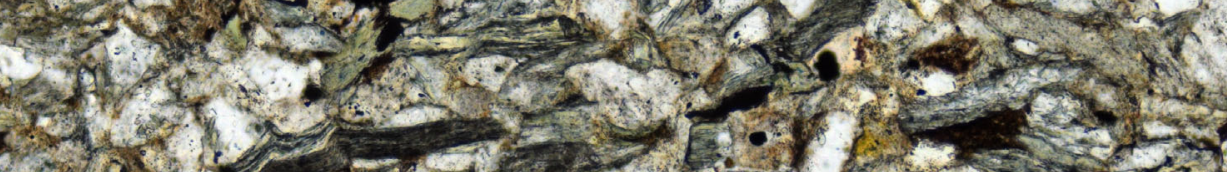
9.1 2 -

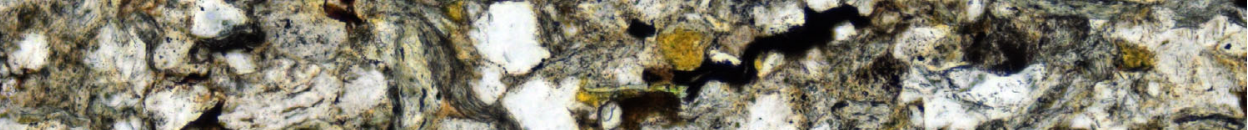

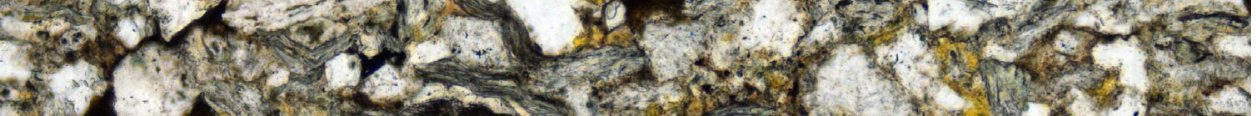

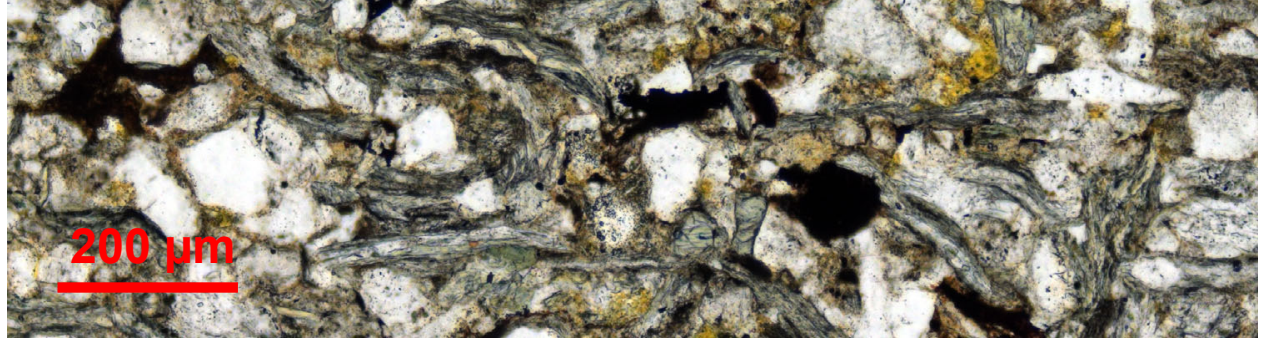

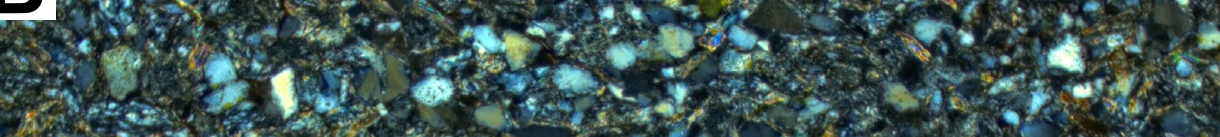

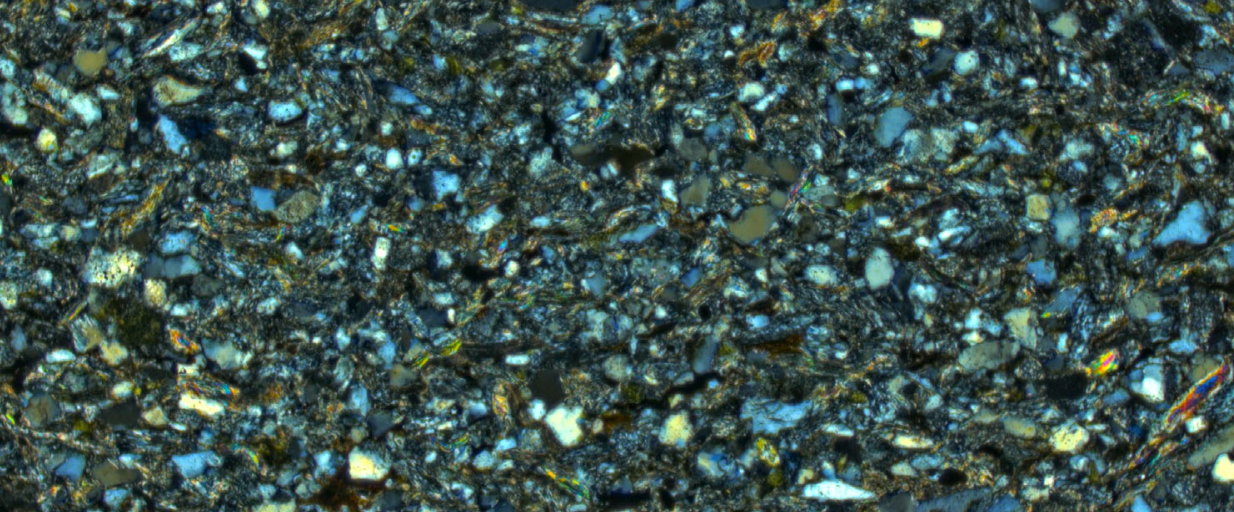

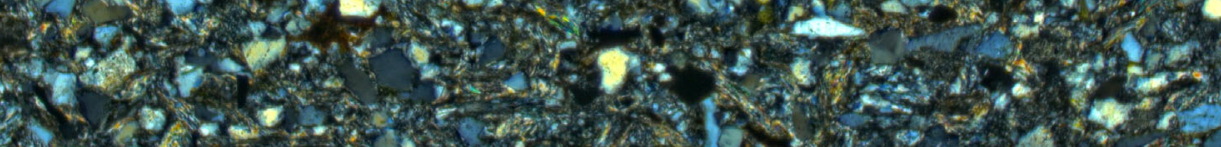

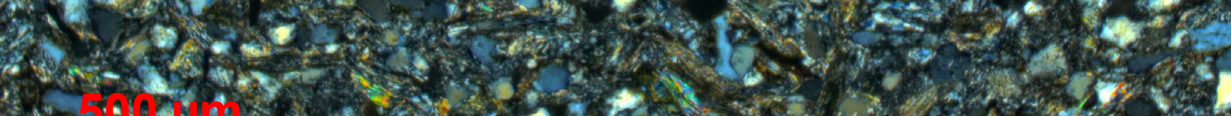

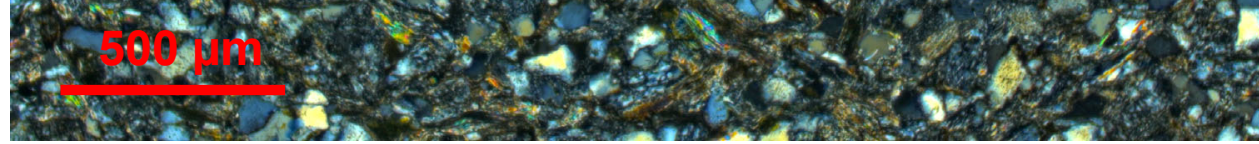

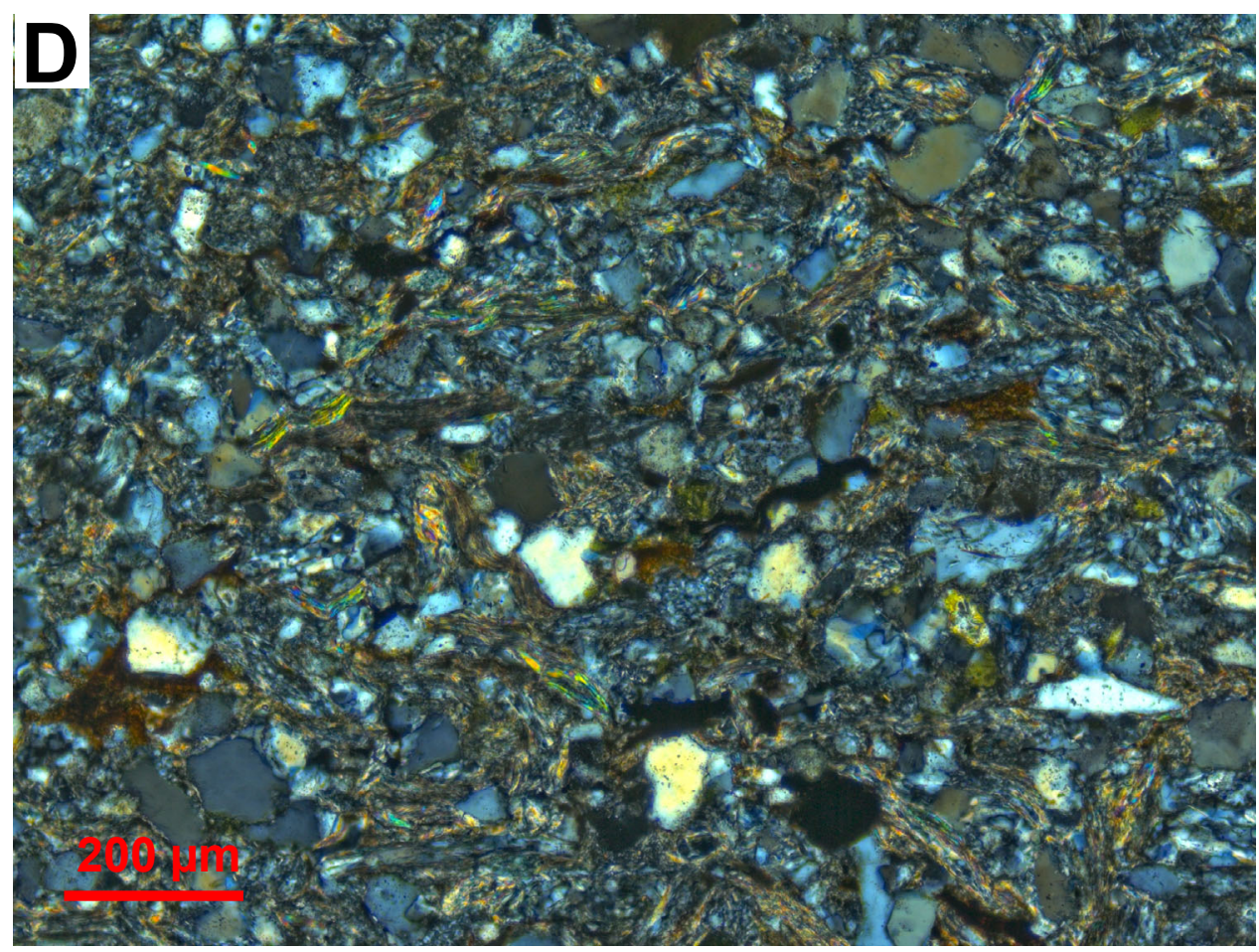


Fig 091 - 19DL029A

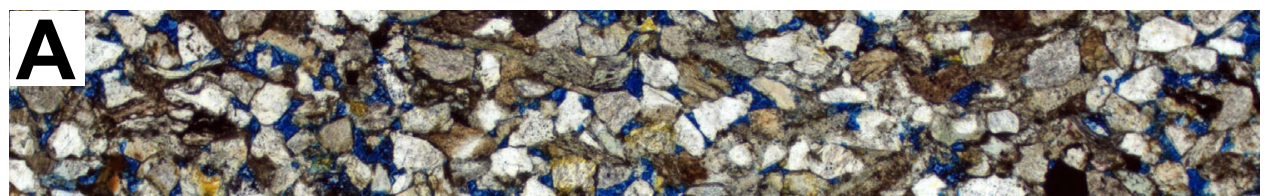

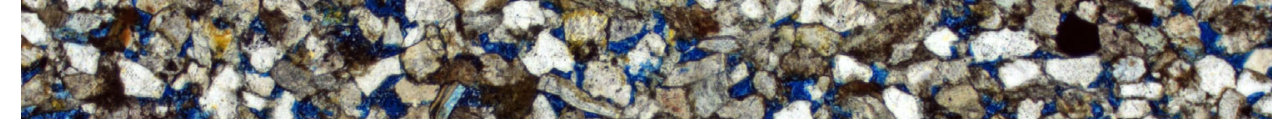

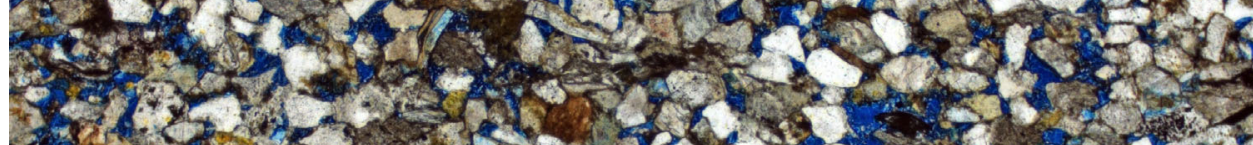

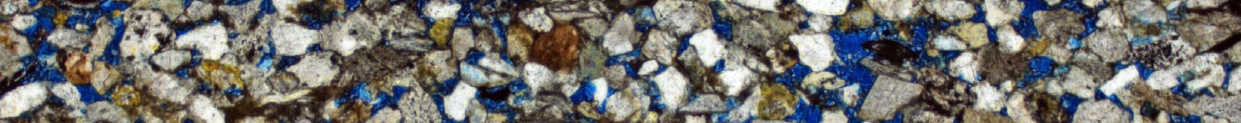

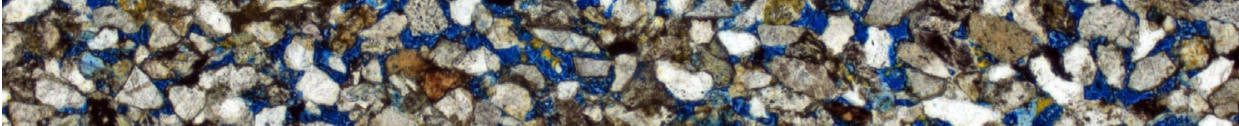

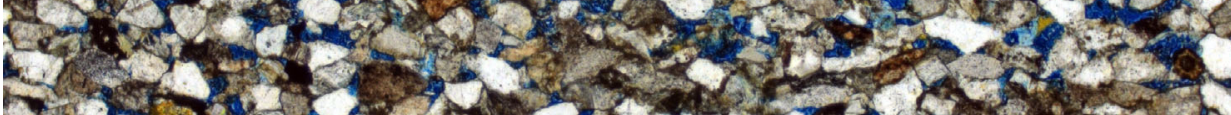

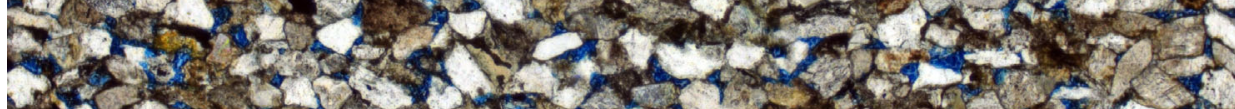

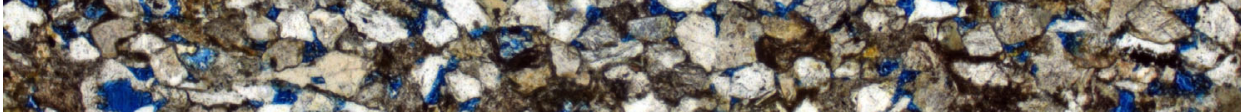

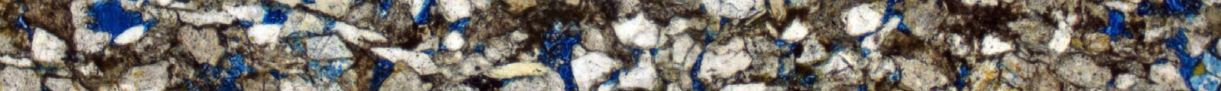

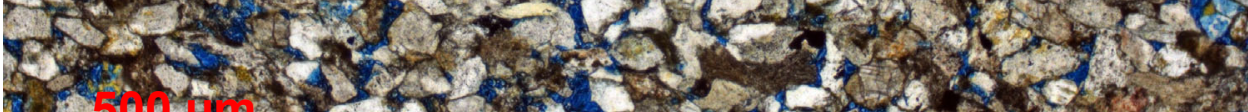

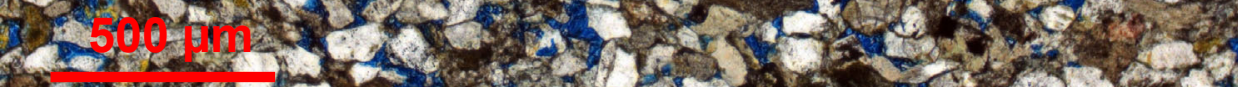

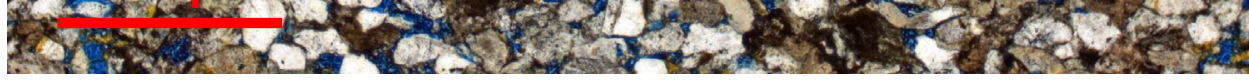

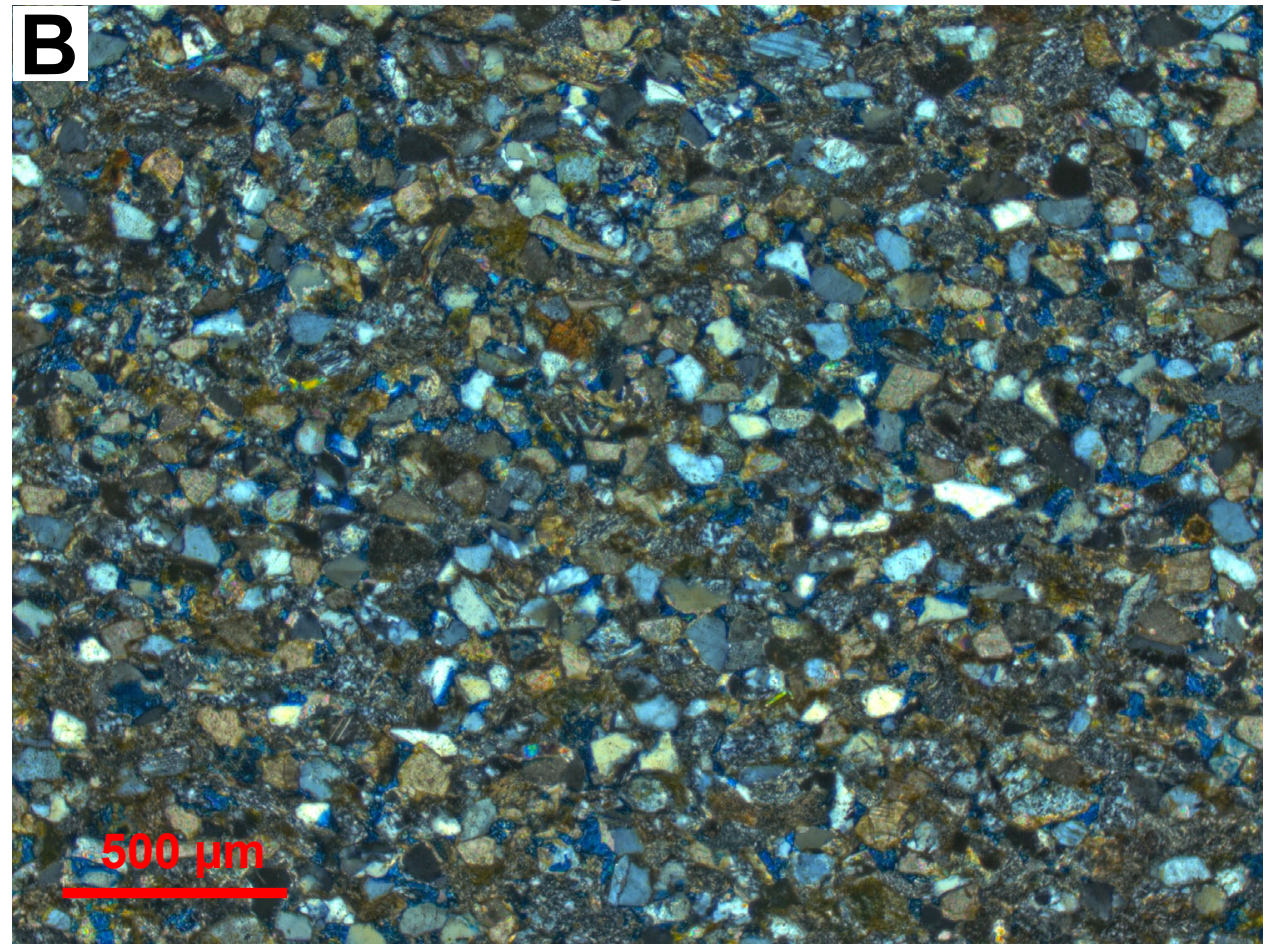
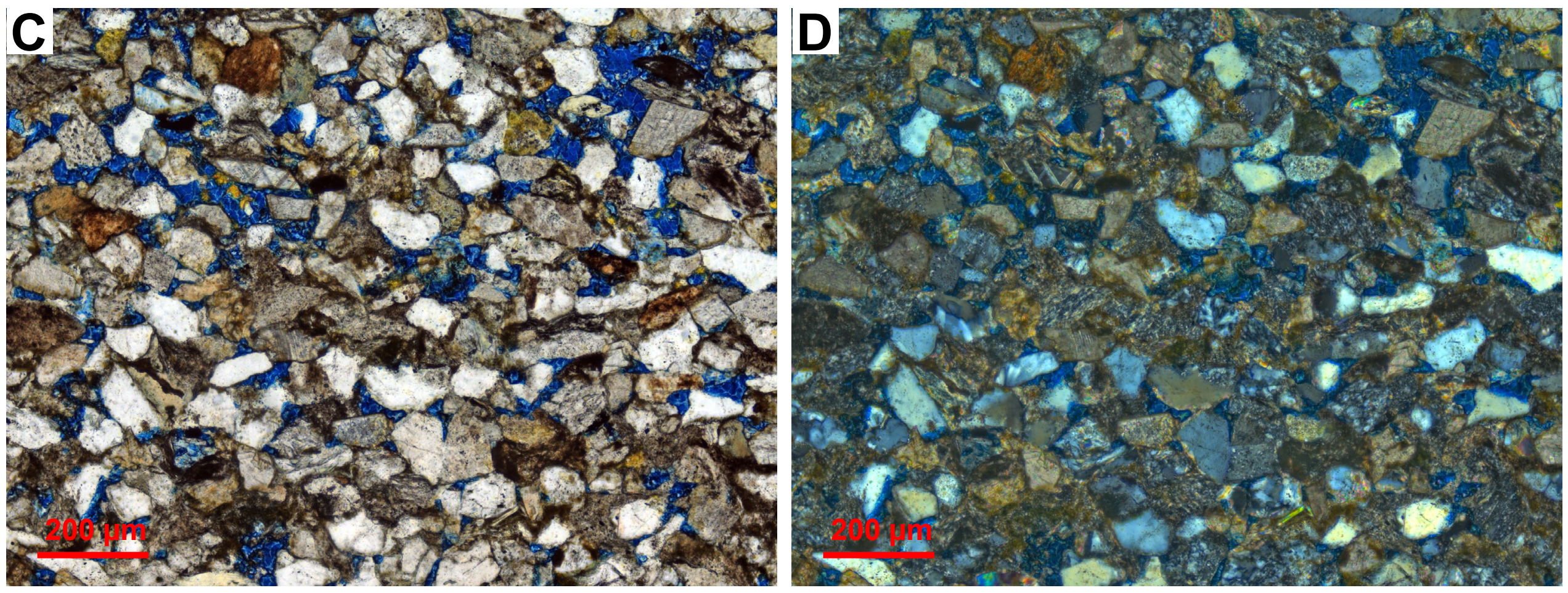
Fig 092 - 19DL033A
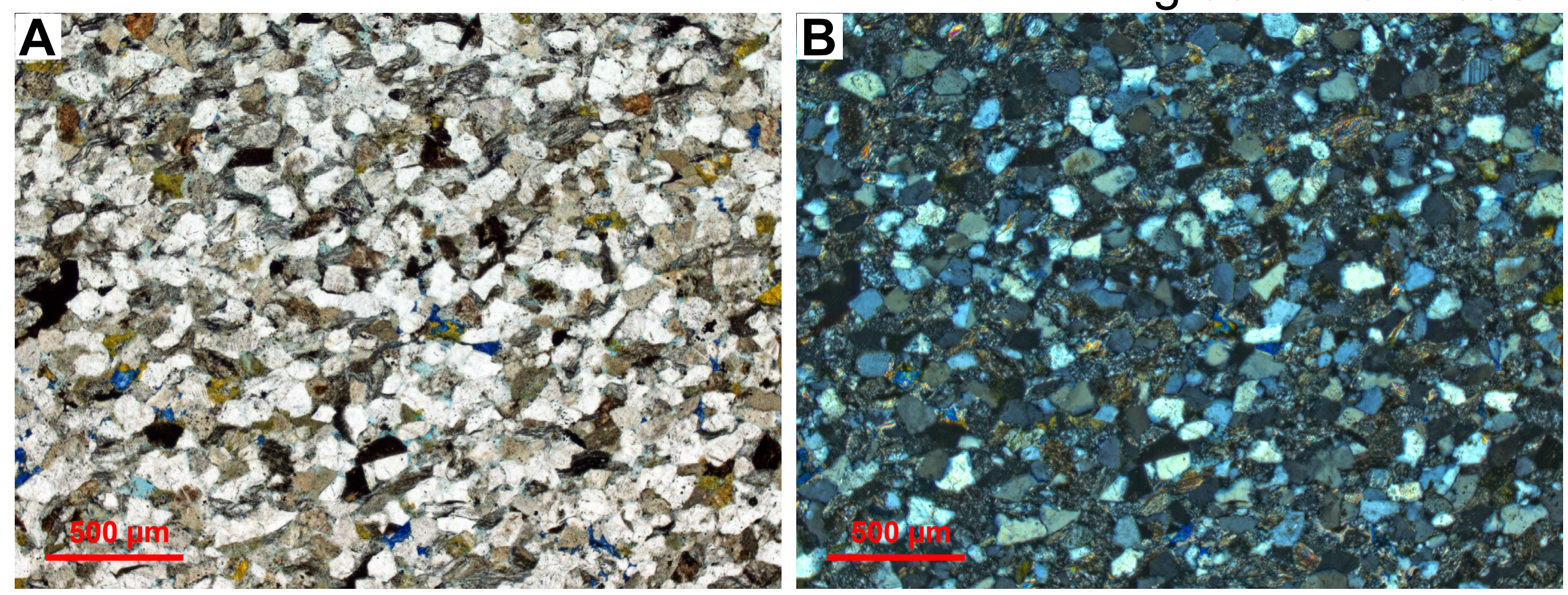
4.

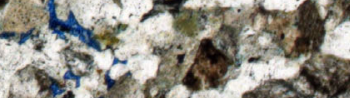

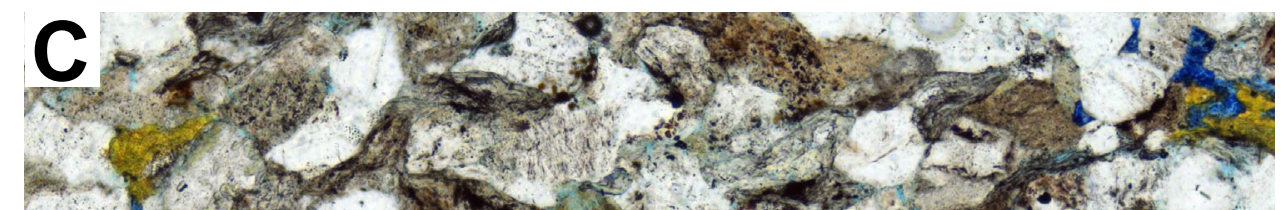

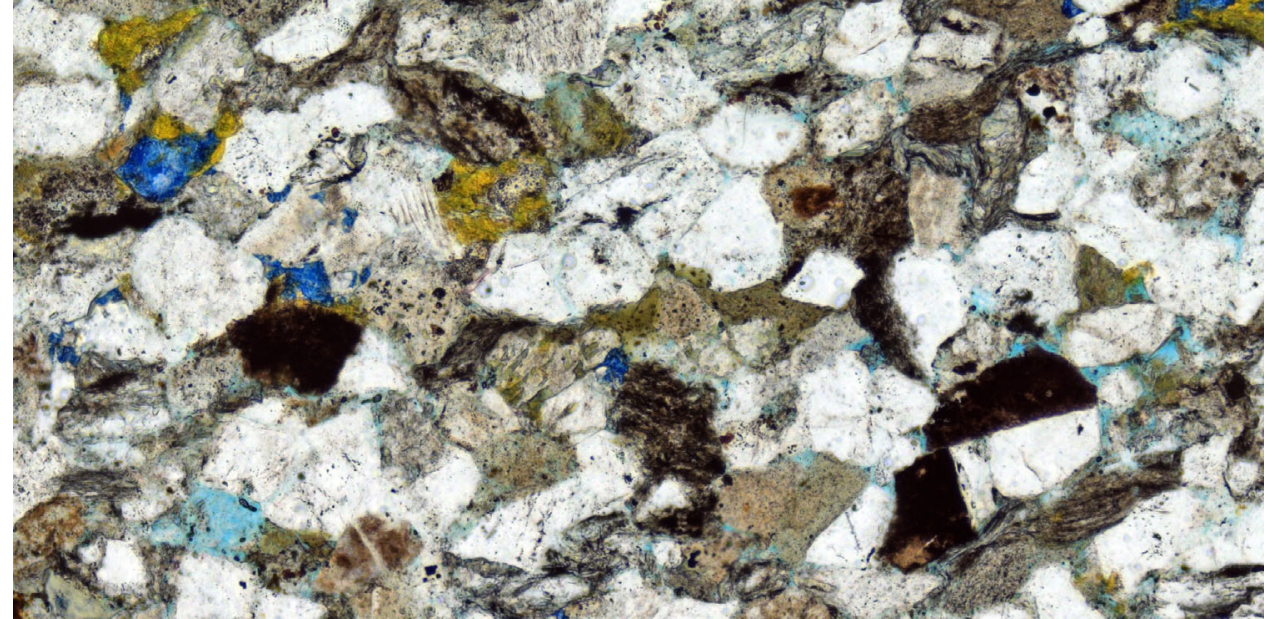

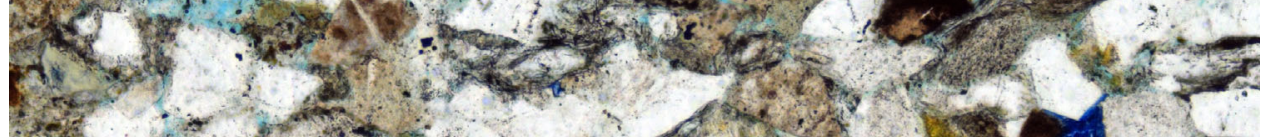

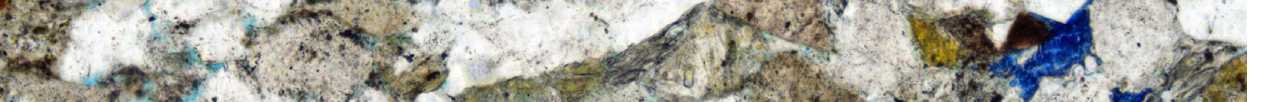

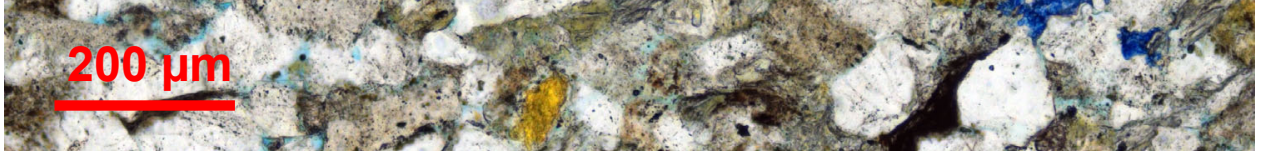

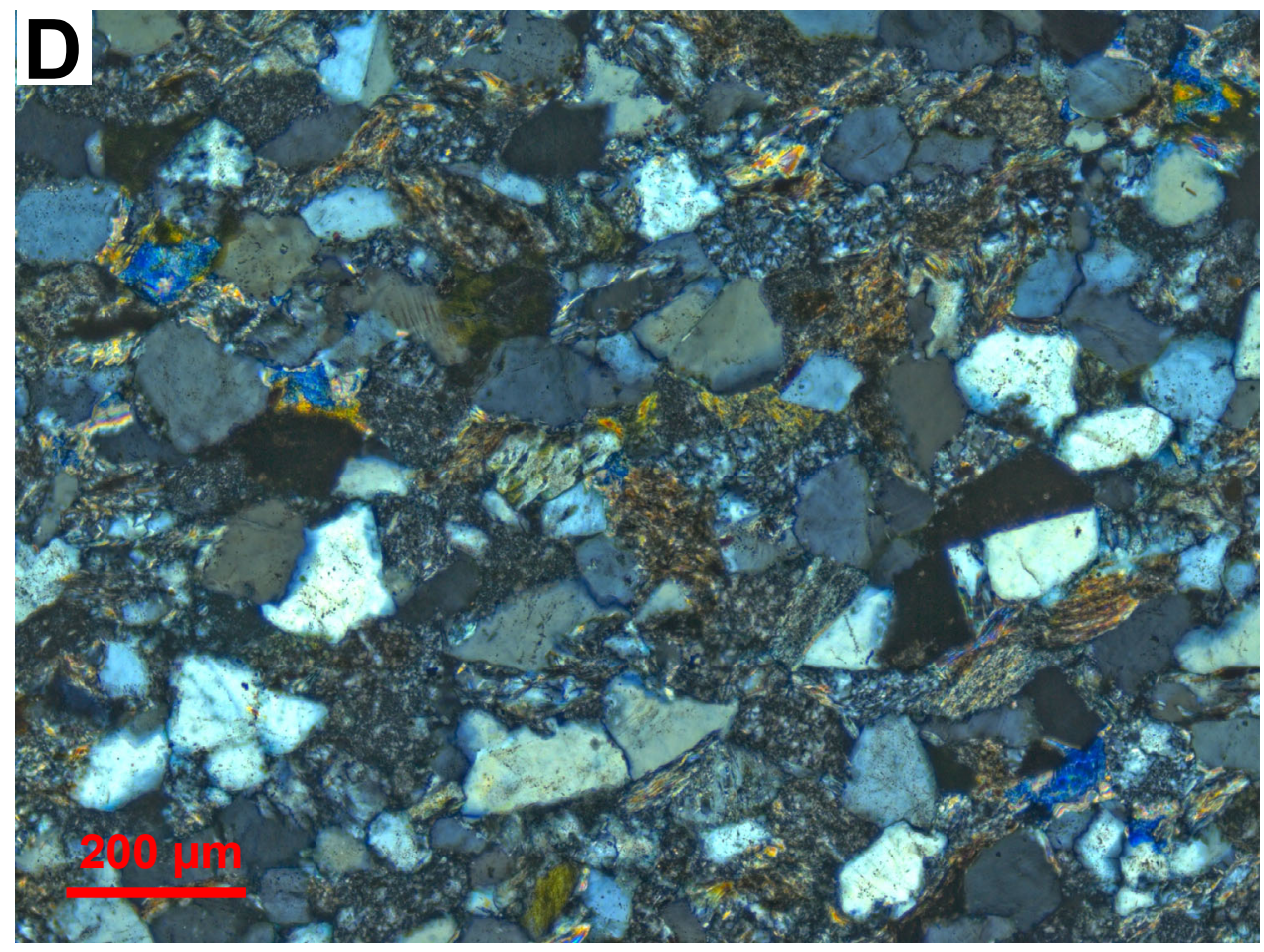


Fig 093 - 06DL007-015.0-2

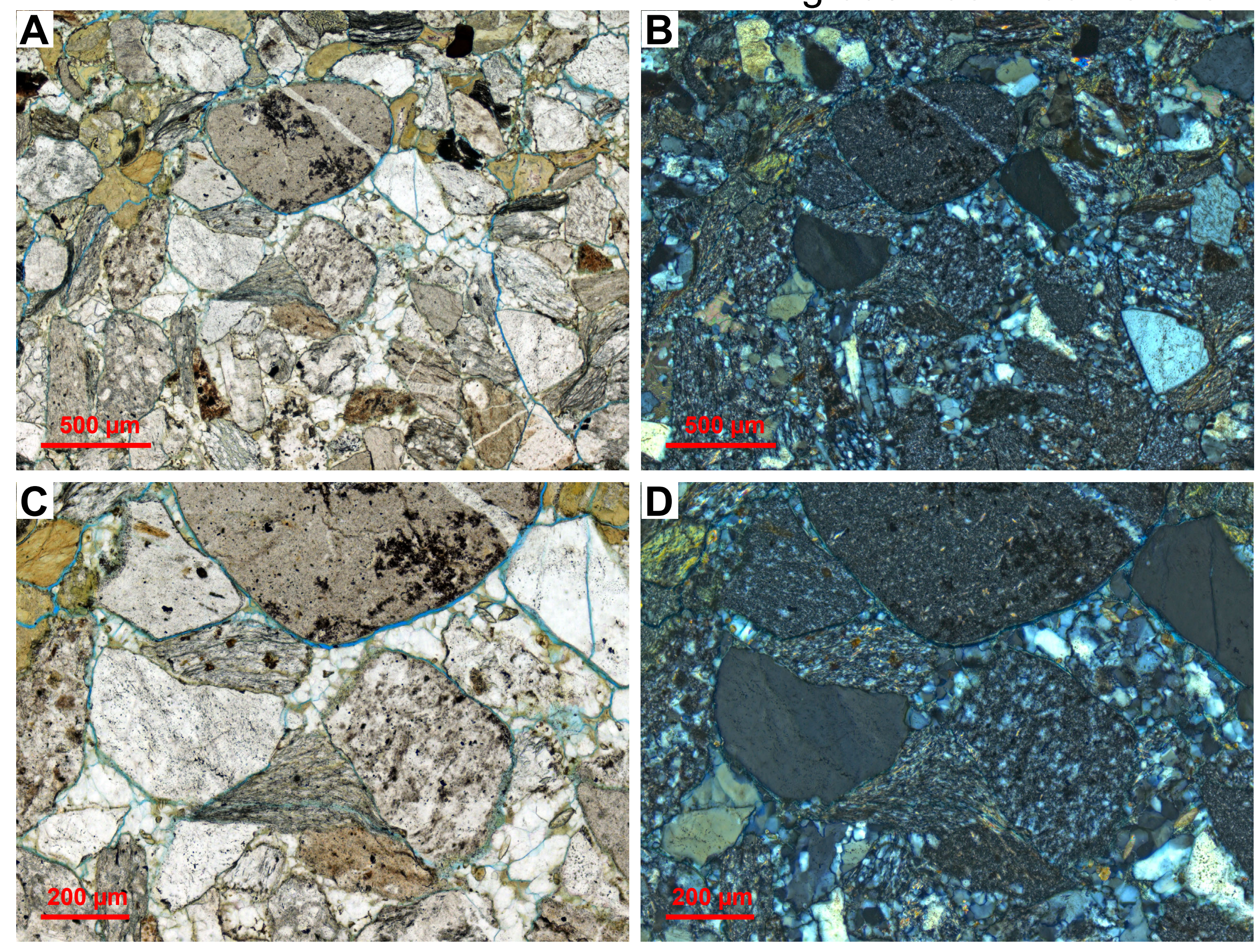


Fig 094 - 06DL007-031.3-2

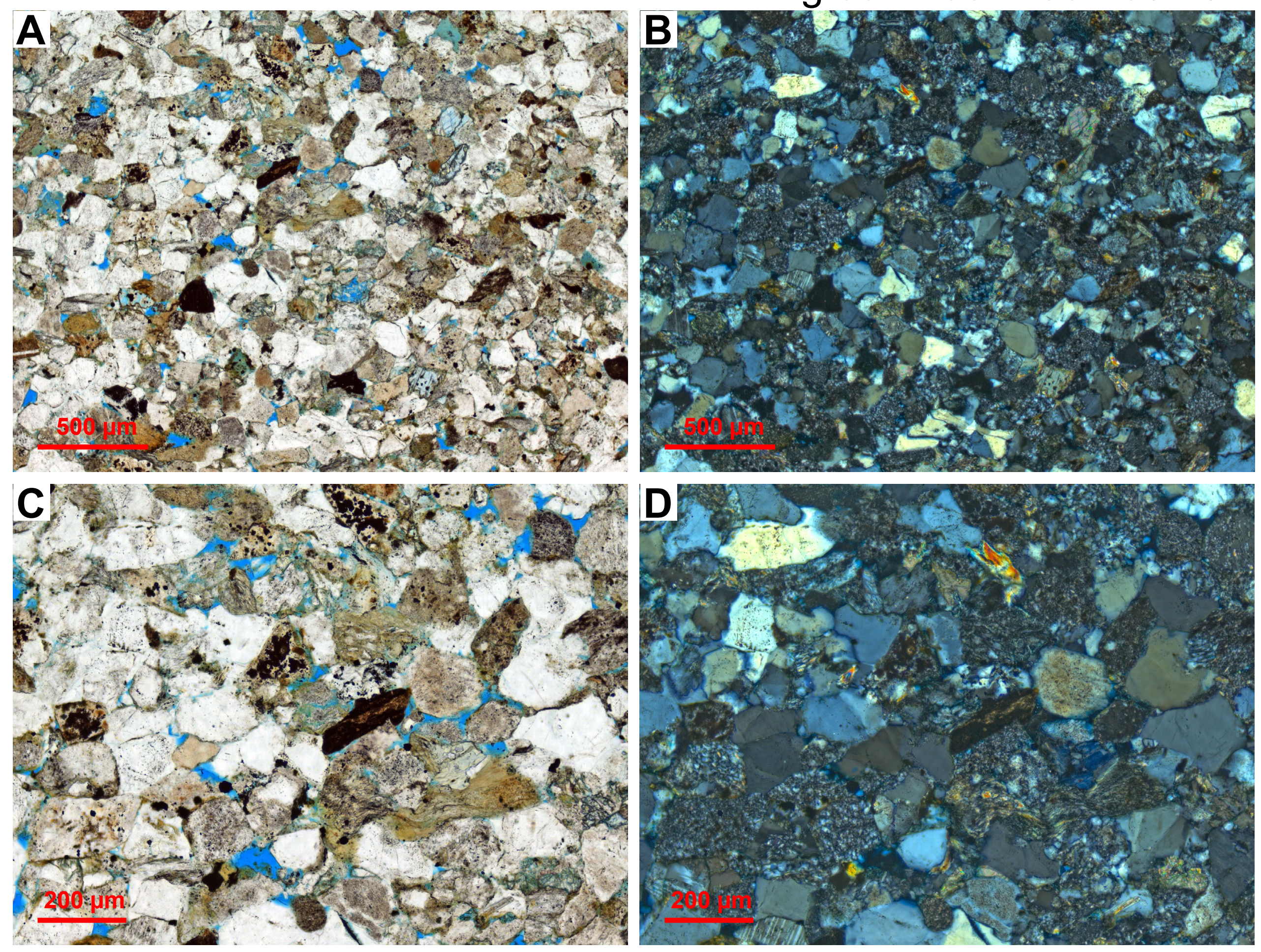


Fig 095 - 06DL007-095.0-2
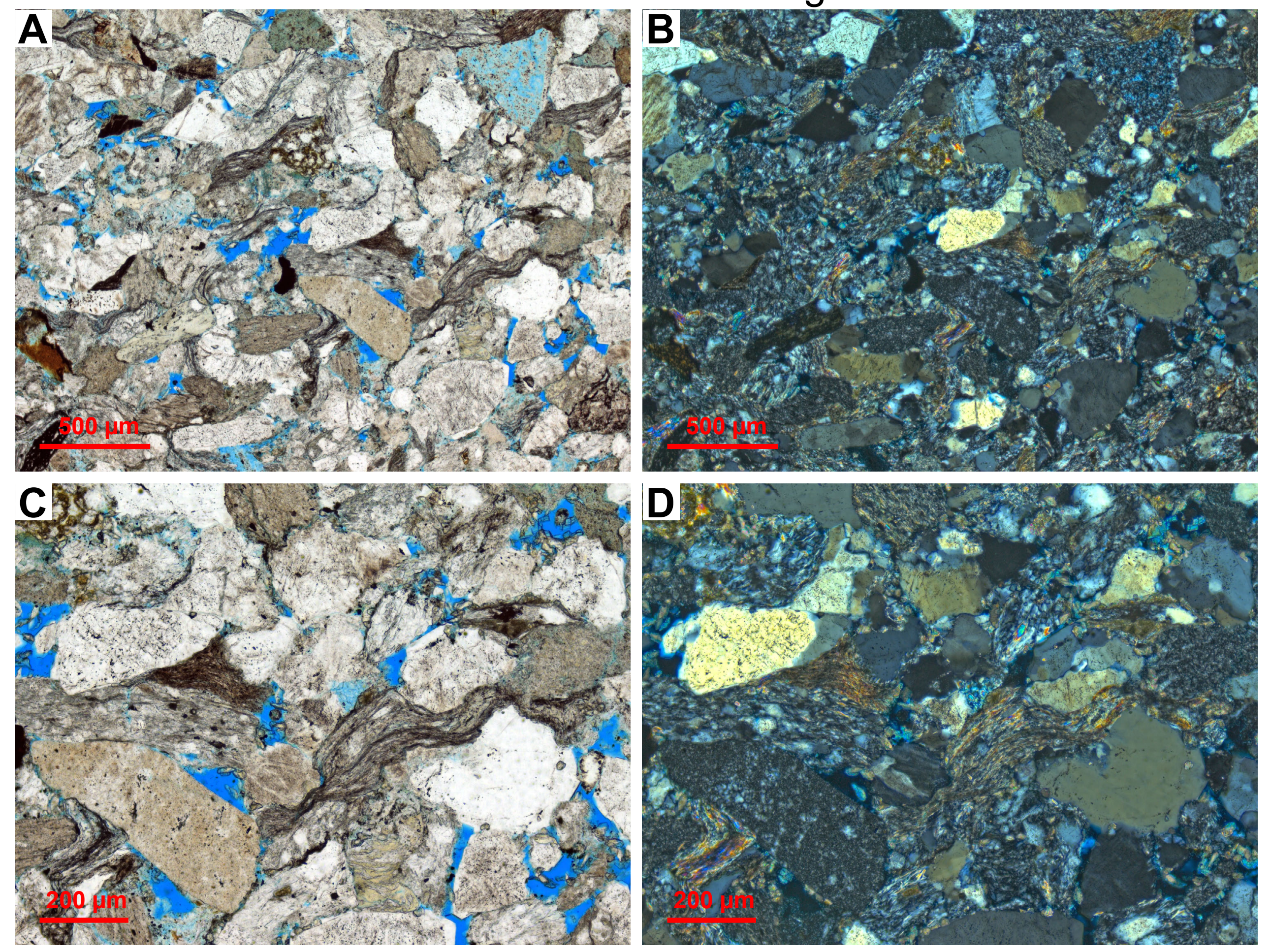
Fig 096 - 06DL007-113.0-2
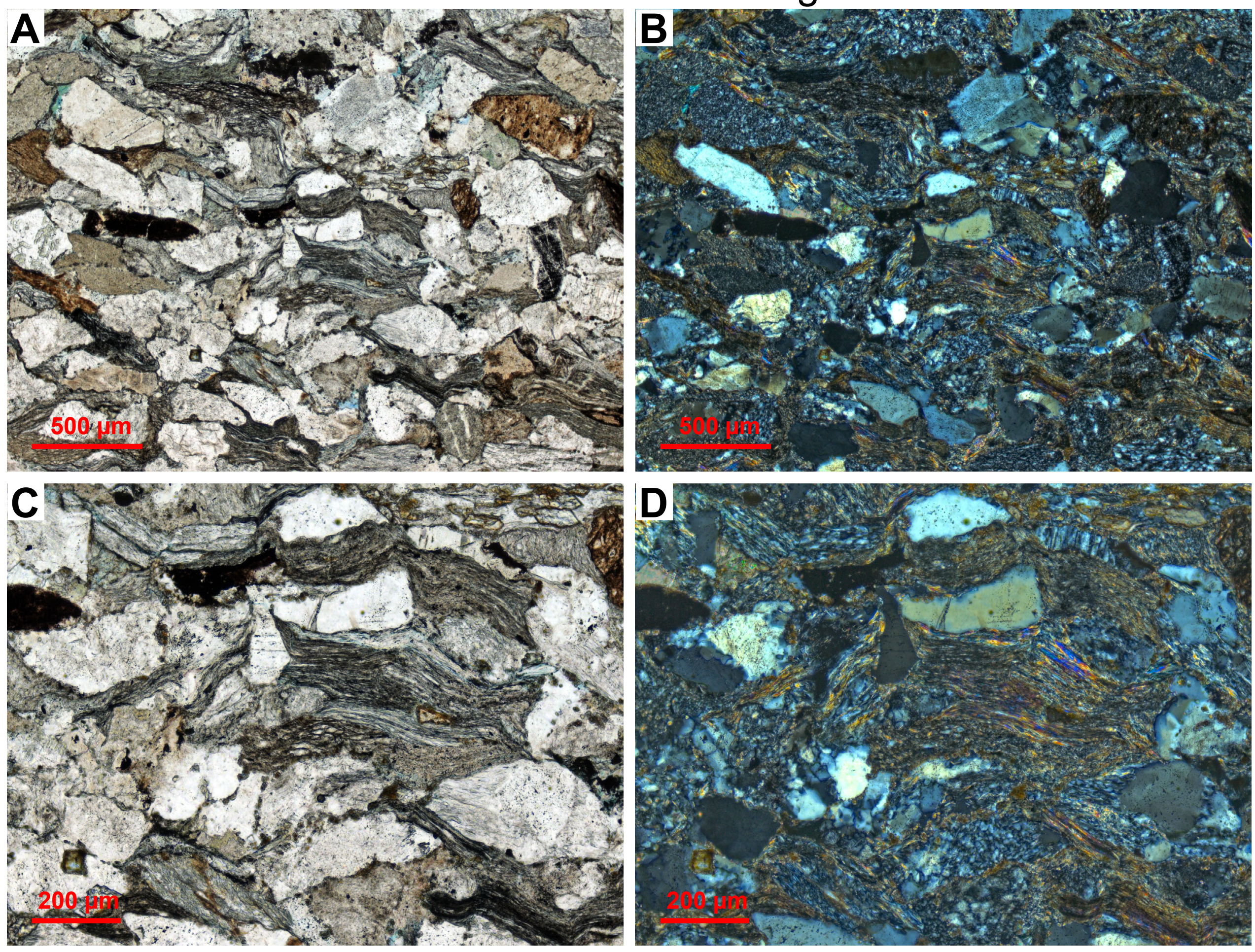
Fig 097 - 06DL003-021.0-2
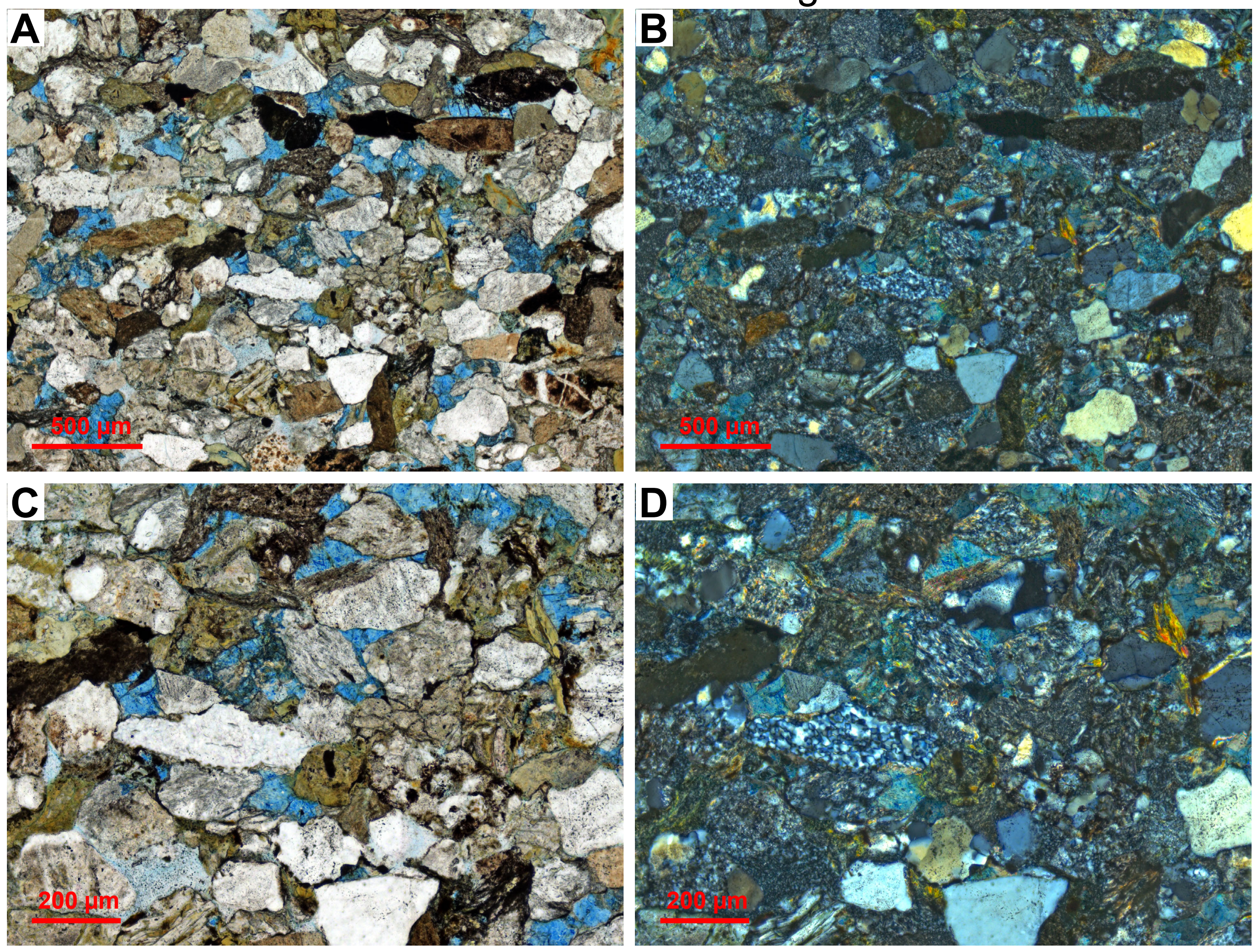
Fig 098 - 06DL003-413.7A
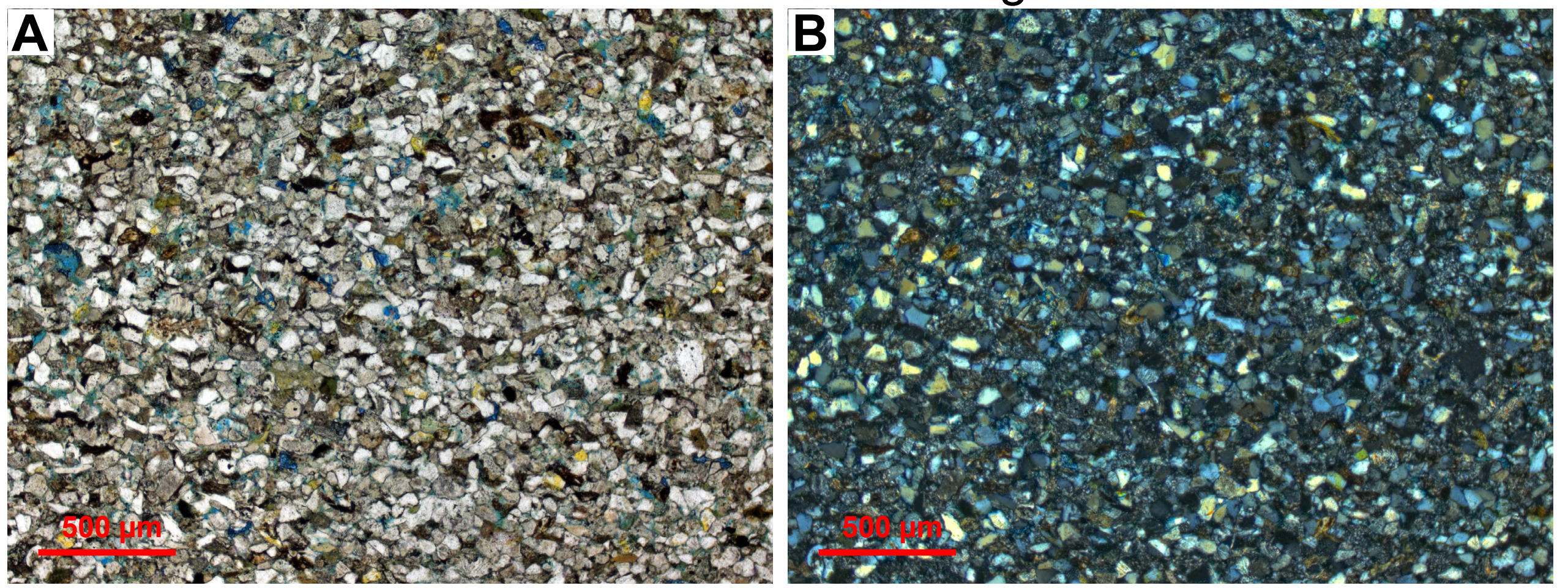

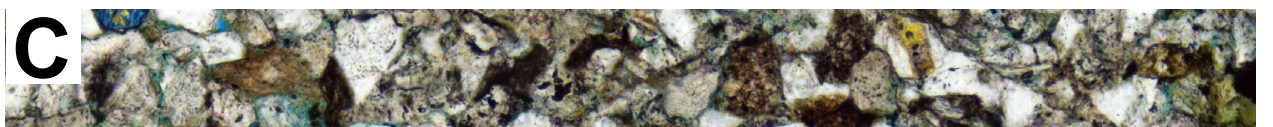

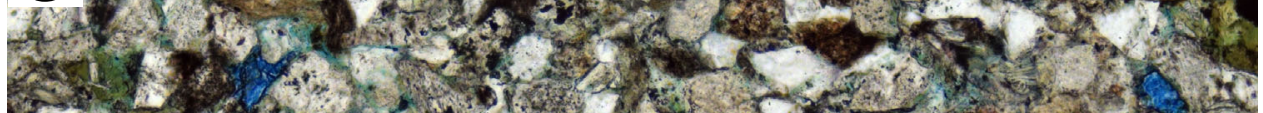

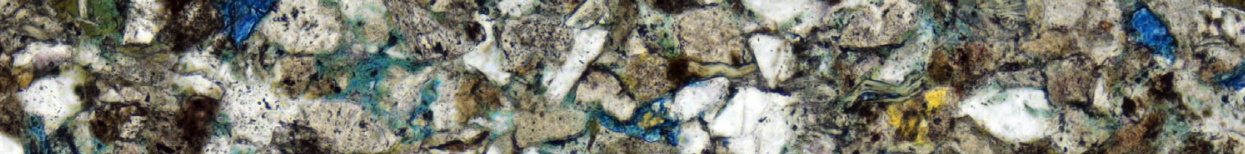

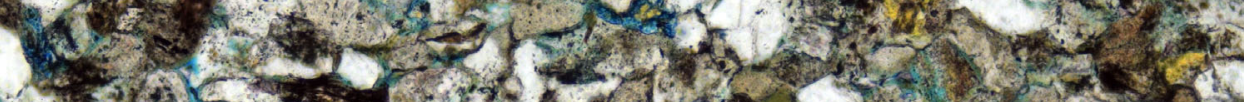

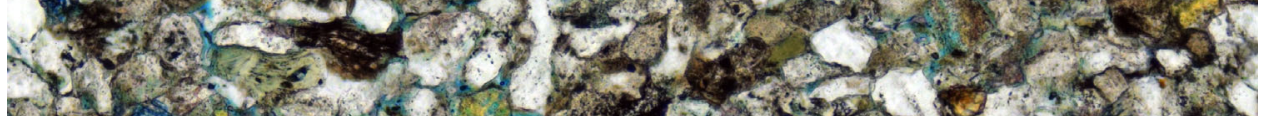

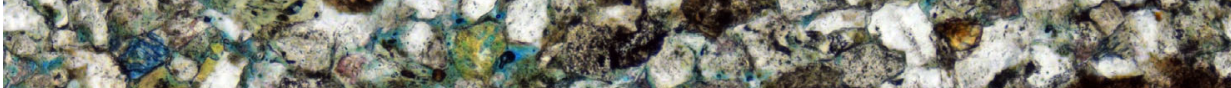
$172 x^{5}-25$ $2+25000^{-1}$ tants 1

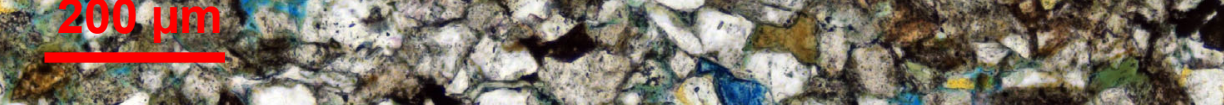

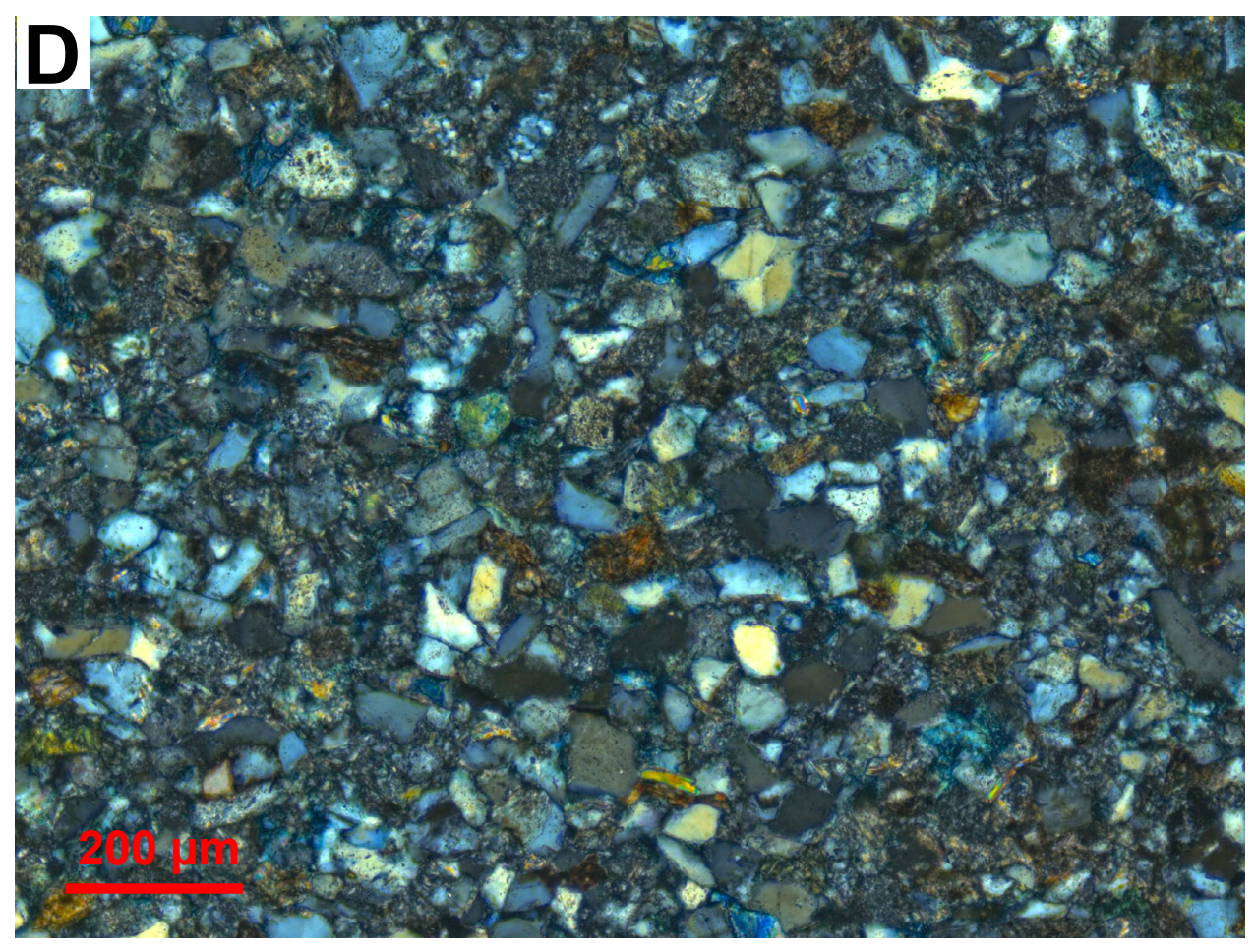


Fig 099 - 06DL003-431.0A
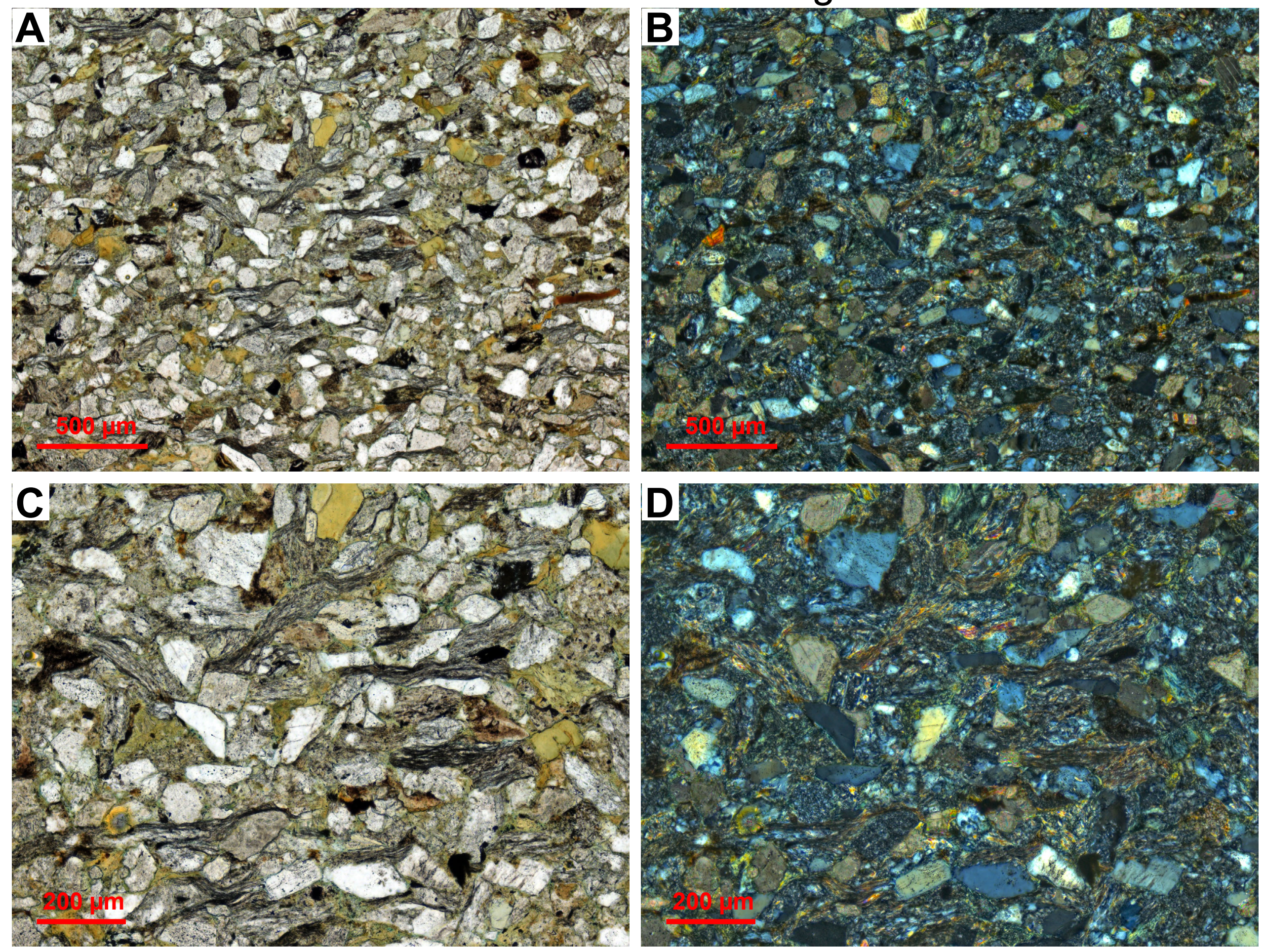
Fig 100 - 06DL003-445.2A

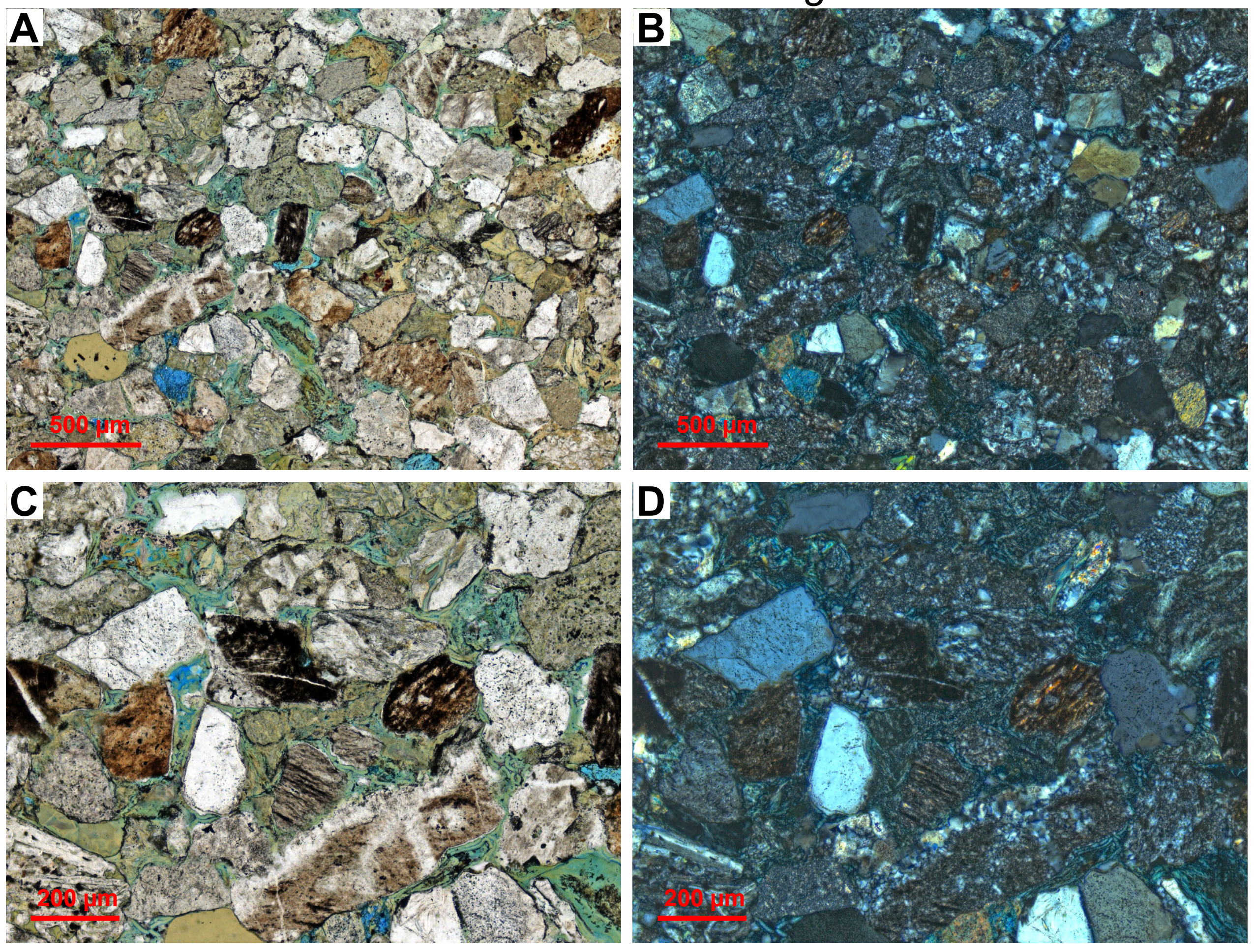


Fig 101 - 06DL003-449.0A

A 5 - 151720

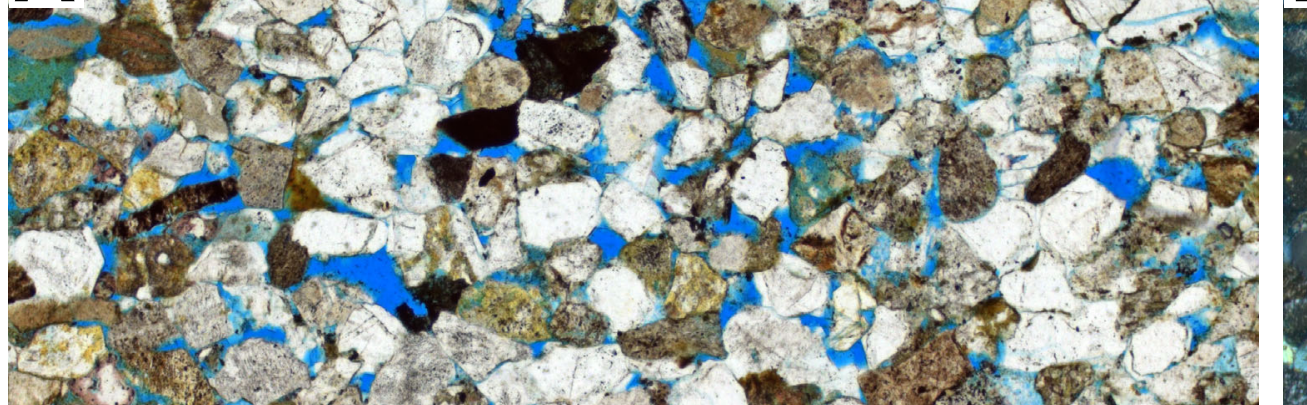

x. 0.120

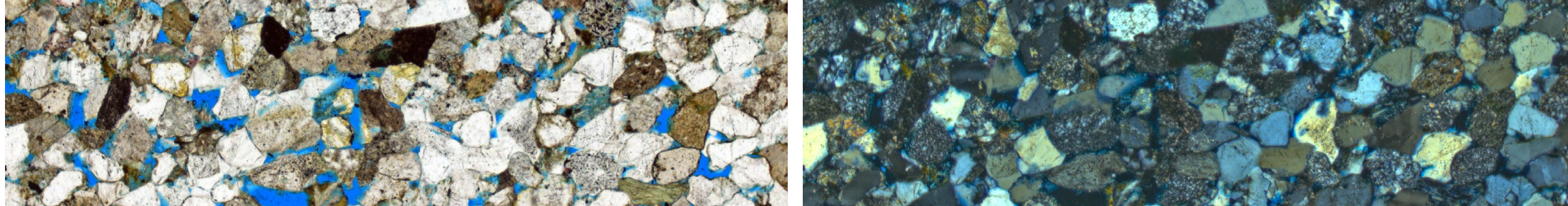

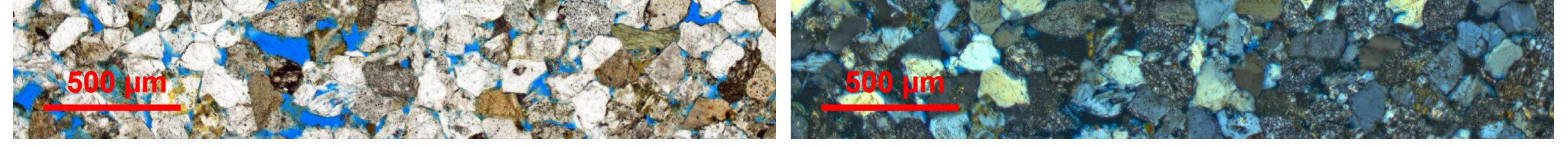

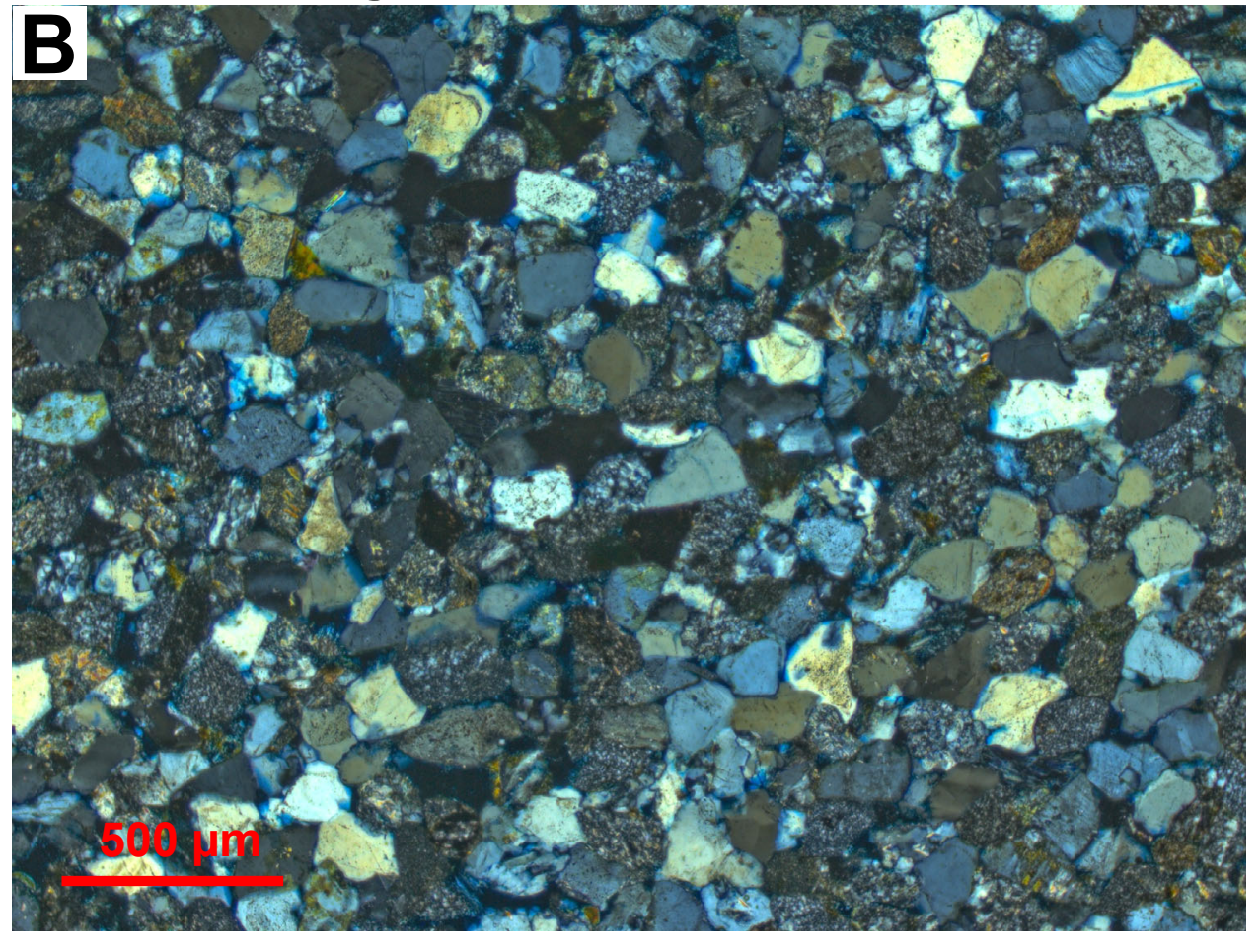

C T 10.127

$x \rightarrow 1$ 1.7.
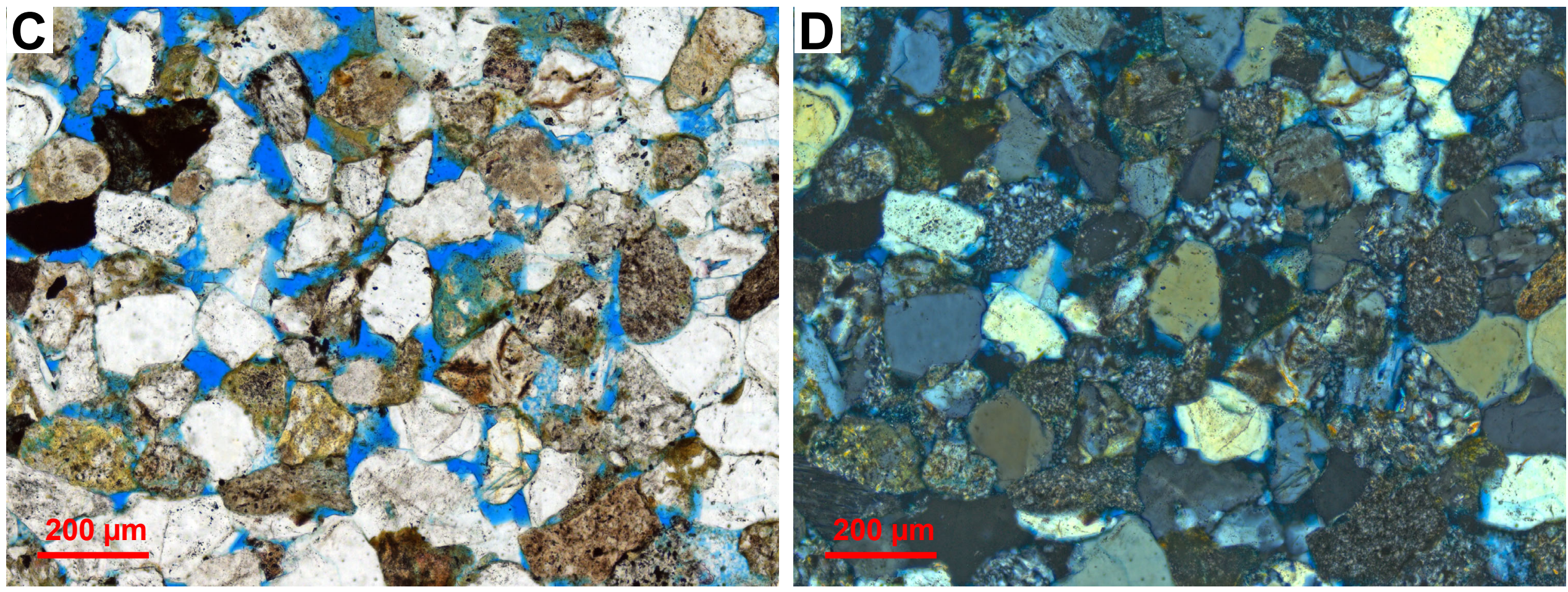
Fig 102 - 06DL005A-2
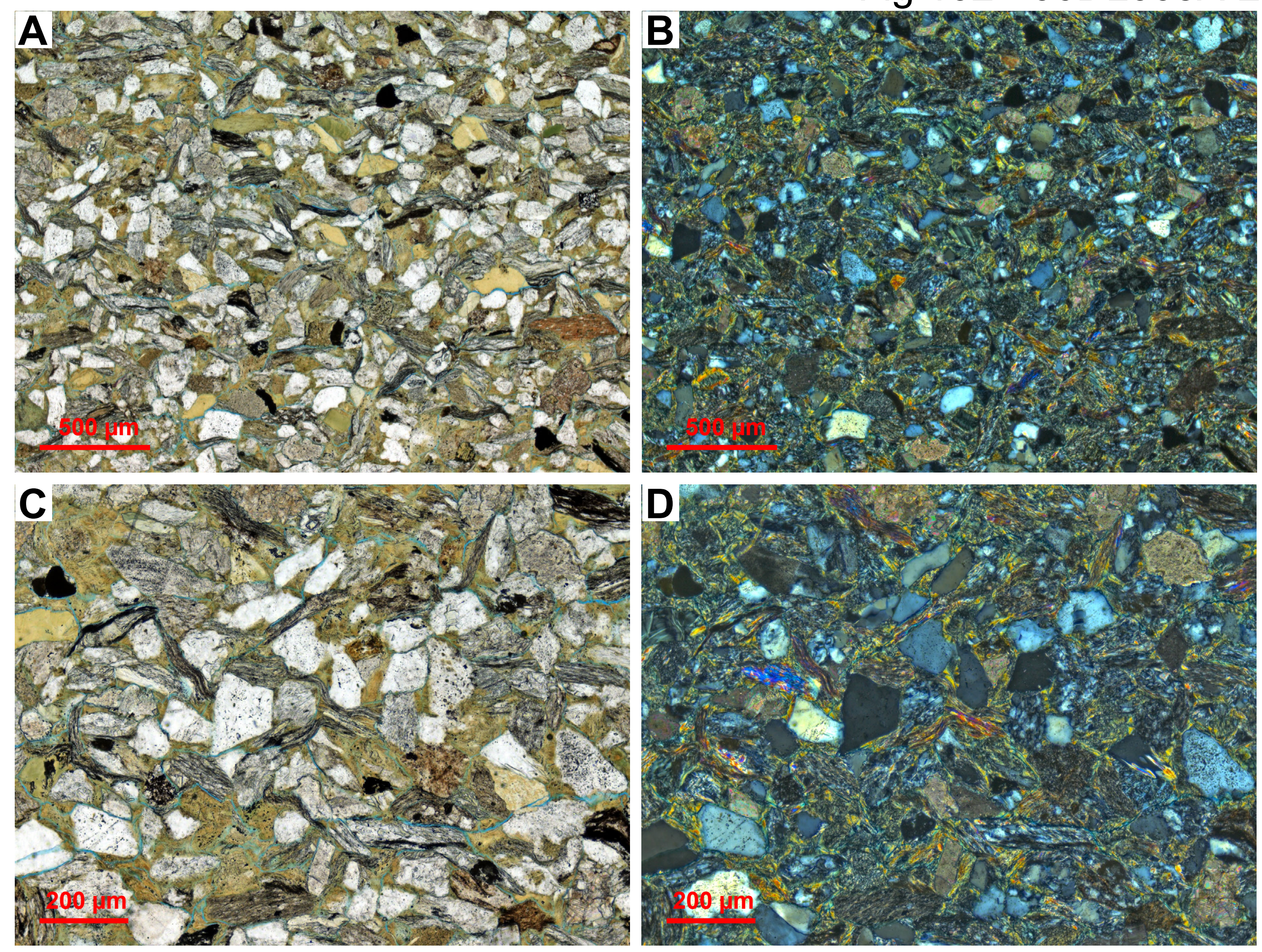


\section{ACKNOWLEDGMENTS}

Funding was provided over the past 22 years by an industry consortium comprised of various companies, including Anadarko Petroleum, BP Exploration, Chevron USA Production Company, ConocoPhillips, Encana, Eni, Oil Search, PetroCanada, Pioneer Resources, Shell Western Exploration and Production, TotalFinaElf, and Unocal; some of these companies no longer exist. Additional funding was provided by the U.S. Geological Survey and the State of Alaska.

\section{REFERENCES}

Boggs, S., Jr., 2009, Petrology of Sedimentary Rocks, $2^{\text {nd }}$ ed.: Cambridge University Press, New York, 600 p. Helmold, K.P., 2016, Sedimentary petrology and reservoir quality of Albian-Cenomanian Nanushuk Formation sandstones, USGS Wainwright \#1 test well, western North Slope, Alaska, in LePain, D.L., ed., Stratigraphic and reservoir quality studies of continuous core from the Wainwright \#1 coalbed methane test well, Wainwright, Alaska: Fairbanks, Alaska, Alaska Division of Geological and Geophysical Surveys Report of Investigations 2016-3-3, p. 37-58.

LePain, D.L., and Helmold, K.P., eds., 2021, Sedimentology and Reservoir Quality of the Nanushuk Formation (Albian-Cenomanian) in Cores from the National Petroleum Reserve-Alaska and Adjoining State Lands to the South: Alaska Division of Geological \& Geophysical Surveys Report of Investigation 2021-4, 78 p. https://doi.org/10.14509/30726

Hurst, A., and Nadeau, P.H., 1995, Clay microporosity in reservoir sandstones: an application of quantitative electron microscopy in petrophysical evaluations: American Association of Petroleum Geologist Bulletin, v. 79, no. 4, p. 563-573.

LePain, D.L., McCarthy, P.J., and Kirkham, R.A., 2009, Sedimentology and sequence stratigraphy of the middle Albian-Cenomanian Nanushuk Formation in outcrop, central North Slope, Alaska: Alaska Division of Geological \& Geophysical Surveys Report of Investigation 2009-1 v. 2, 76 p., 1 sheet. https://doi.org/10.14509/19761

Nadeau, P.H., and Hurst, A., 1991, Application of back-scattered electron microscopy to the quantification of clay mineral microporosity in sandstone: Journal of Sedimentary Petrology, v. 61, no. 6, p. 921-925.

Wilson, F.H., Hults, C.P., Mull, C.G., and Karl, S.M., 2015, Geologic Map of Alaska: U.S. Geological Survey Scientific Investigations Map 3340, pamphlet 197p., 2 sheets, scale 1:1,584,000, http://pubs.er.usgs.gov/pubblication/sim3340 (on DVD).

Wilson, M.D., and Pittman, E.D., 1977, Authigenic clays in sandstones: recognition and influence on reservoir properties and paleoenvironmental analysis: Journal of Sedimentary Petrology, v. 47, no. 1, p. 3-31. 